\title{
Evaluating Pedestrian Head Sub-system Test Procedure against Full- scale Vehicle-Pedestrian Impact Using Numerical Models
}

\author{
A dissertation presented to the faculty of the \\ School of Engineering and Applied Science, \\ University of Virginia \\ In Partial Fulfillment \\ of the Requirements for the Degree \\ Doctor of Philosophy in Mechanical and Aerospace Engineering \\ by
Huipeng Chen
}

May 2017 
The dissertation is submitted in partial fulfillment

Of the requirements for the degree of

Doctor of Philosophy in Mechanical and Aerospace Engineering

Author

The dissertation has been read and approved by the examining committee:

Matthew B. Panzer (Dissertation Advisor)

Jeff R. Crandall (Committee Chair)

Jason R. Kerrigan

Thomas Hartka

Jason L. Forman

Accepted for the School of Engineering and Applied Science:

Dean, School of Engineering and Applied Science

May 2017 


\begin{abstract}
$\underline{\text { Abstract }}$
Head injury is the primary cause of pedestrian fatalities. To address this problem, the simplified sub-system test procedure has been used to distinguish vehicle stiffness in recent decades. Compared to the full-scale dummy test, the sub-system test has the advantage in terms of cost, repeatability, and testing coverage. However, the sub-system test methodology has long been criticized for oversimplification and for missing headneck interaction, vehicle pre-deformation, and head rotational kinematics, which make the ability of the component test to represent the real full-scale impact questionable. As a result, a potential problem may exist because vehicle design is optimized towards the sub-system test but may not necessarily provide the best protection in real pedestrian accidents. The goal of this dissertation is to study the relationship between the impactor responses of sub-system test with the head response in full-scale pedestrian impact. This process involved multiple steps: 1) evaluate the biofidelity of the PFEM; 2) compare the impactor response in the sub-system test and head response in the full-scale impact; 3) test the hypothesis that vehicle design variations have different influences on the impactor response and head response; and 4) investigate the factors that may contribute to the difference between the component test and the full-scale impact. The results of this work demonstrate limitations in the sub-system test that prevent it from reproducing the full-scale head response, even with matched input kinematics. Many of the limitations of sub-system tests were focused on the lack of vehicle hood pre-deformation caused by the
\end{abstract}


pedestrian torso. The methodology and results of this dissertation could provide reference for improvements to future vehicle safety design and pedestrian regulation development. 


\section{Acknowledgments}

I would first and foremost like to thank my wife, Xiangqing, for all her love, support, and taking care of our daughter during my graduate studies. I would also like to express my gratitude to the faculty, staff, and graduate students of the Center for Applied Biomechanics for their collaboration at every stage of this research. Specifically, I would like to thank my advisor Dr. Matthew Panzer for his thoughtful planning, always clear mind, and all his time spent on my project study and dissertation process; my previous advisor Dr. Jeff Crandall, for his brilliant insights, and deep and critical thinking. Also, I would like to thank Dr. Jason Kerrigan, Dr. Jason Forman, and Dr. Thomas Hartka for their knowledge, suggestions and critical thinking on my dissertation; Dr. David Poulard for his time on discussing the finite element modeling and paper reviewing; Dr. Taewung Kim, for his time on discussing the Multi-body dynamics and paper reviewing, and Lastly, I am very grateful to those researchers and graduate students, Bingbing Nie, Gwansik Park, Varun Bollapragada, Gabler Lee, Taotao Wu, and the rest of my friends in $\mathrm{CAB}$ for their time on the discussion about my studies. 


\section{Table of Contents}

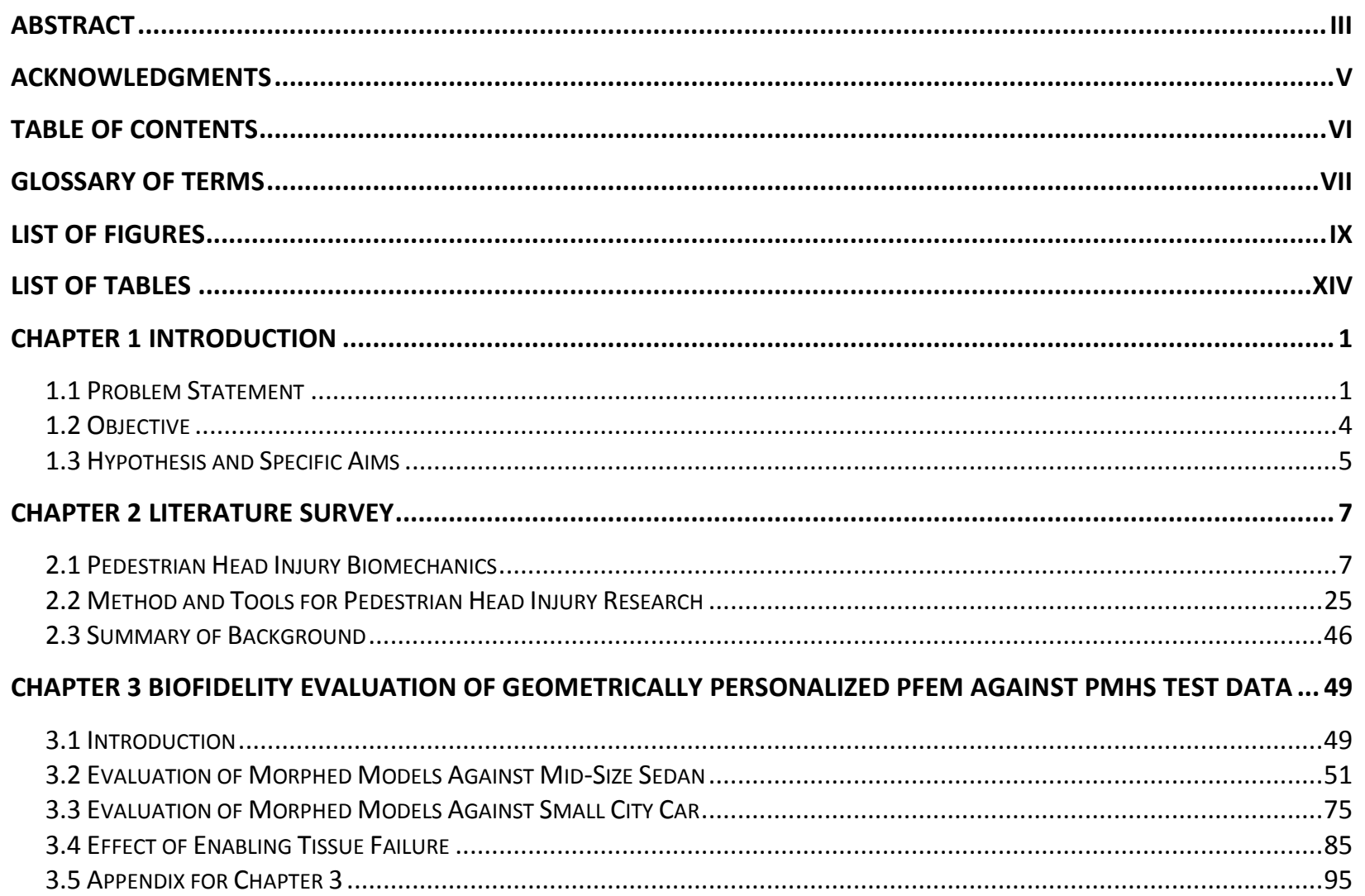

CHAPTER 4 EVALUATING PEDESTRIAN SUB-SYSTEM TEST PROCEDURE AGAINST FULL-SCALE VEHICLE-

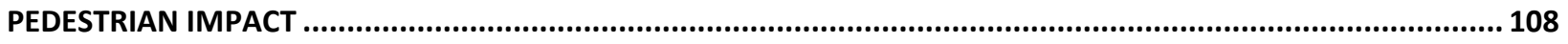

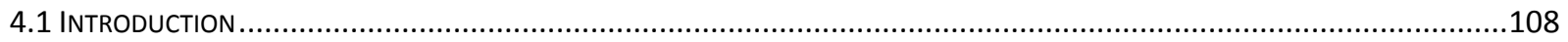

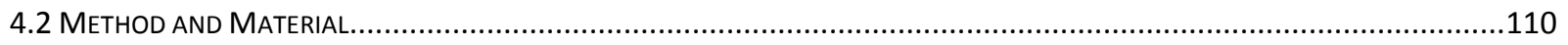

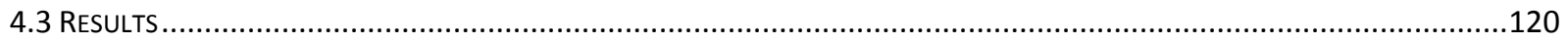

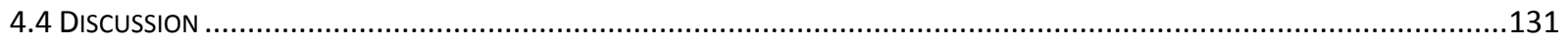

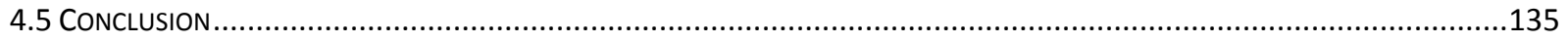

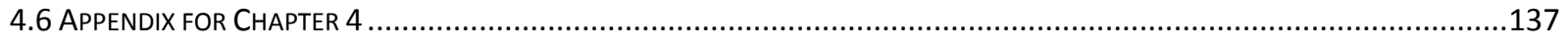

CHAPTER 5 EFFECT OF FACTORS ON PEDESTRIAN HEAD RESPONSE ................................................................. 151

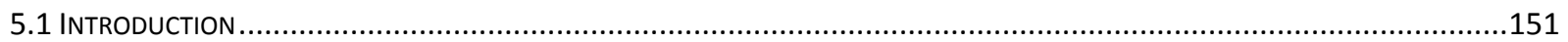

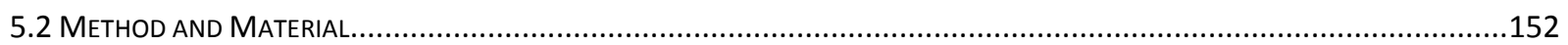

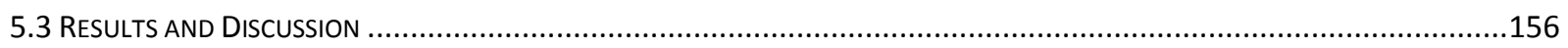

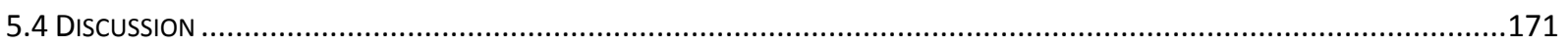

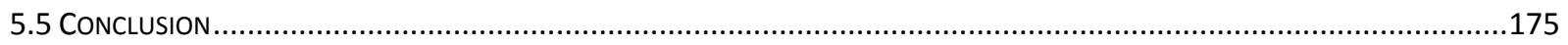

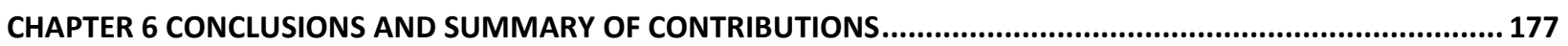

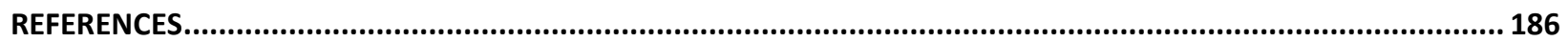




\section{Glossary of Terms}

ACL - Anterior Cruciate Ligament

AIS - The Abbreviated Injury Scale

Anthropometry - Individual or statistical measurements of the human body as a whole, or of parts of the human body, e.g., total height, total mass or body part dimensions.

Biofidelity - Human likeness

BLE - Bonnet leading edge as defined by EEVC 98

BrIC - abbreviation for Rotational Brain Injury Criterion

CORA - The CORrelation and Analysis Method

DM - Deterministic method

EEVC - European Enhanced Vehicle-safety Committee

Euro-NCAP - European New Car Assessment Programme

FE - Finite Element

Full-scale - In this context, full-scale experiments or simulations refer to complete pedestrian tests or simulations where the whole human body is engaged as opposed to component tests

GHBMC - Global Human Body Models Consortium

GTR - Global Technical Regulation

HIC - Head Injury Criteria

IHRA - International Harmonised Research Activities

ISO - International Organization for Standardization

IRTAD - International Road Traffic and Accident Database

LCL - Lateral Collateral Ligament 
MB - Multi-Body

MCL - Medial Collateral Ligament

NHTSA - National Highway Traffic Safety Administration

PCL - Posterior Cruciate Ligament

PCDS - Pedestrian Crash Data Study

PFEM - Pedestrian Finite Element Model

PM - Probabilistic method

PMHS - Post Mortem Human Subject, human cadaver

TBI - Traumatic brain injury

THUMS - Total Human Model for Safety, developed by TOYOTA MOTOR CORPORATION

in cooperation with Toyota Central R\&D Labs Inc

WAD - Wrap Around Distance, length measurement from the ground to the impact location of any specific body part on a vehicle, as defined by EEVC 98.

WHO - World Health Organization

50th-percentile male - the hypothetical average man at the center of the anthropometric spectrum 


\section{List of figures}

Figure 1.1 Distribution of pedestrian injuries in vehicle-pedestrian accidents (adapted from Crandall et al. 2002) .......2

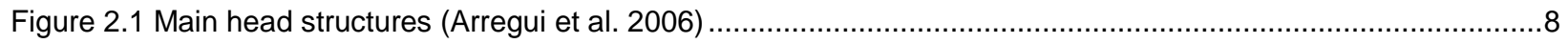

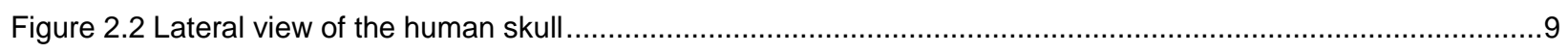

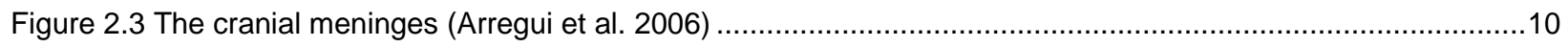

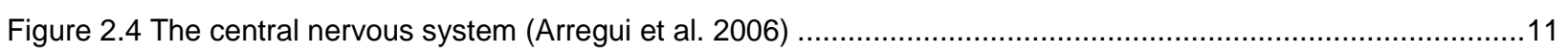

Figure 2.5 Cumulative vehicle impact speed distribution (Chen et al. 2009) …....................................................12

Figure 2.6 Frequency of vehicle impact speed in pedestrian accidents (Chen et al. 2009).....................................12

Figure 2.7 Frequency of vehicle impact speed in pedestrian accidents (Rosén et al. 2009) ...................................13

Figure 2.8 The fatality risk as a function of impact speed for adult pedestrians hit by the front of a passenger car. The dotted curves show approximate 95\% confidence limits. (Rosén et al. 2009) …….....................................13

Figure 2.9 Head injury types and injury causes (Mizuno and Kajzer, 2000)......................................................19

Figure 2.10 Head injuries versus injury mechanisms (Gennarelli et al. 1989; Arregui 2006) ..................................22

Figure 3.1 Initial positions. Lateral and posterior view of subject 1 (left) and subject 2 (right) ..................................53

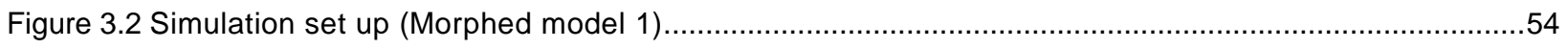

Figure 3.3 Pedestrian impact sequences of PMHS and models (subject 1) ......................................................58

Figure 3.4 Pedestrian impact sequences of PMHS and models (subject 2). ….............................................58

Figure 3.5 Time history of resultant velocity on each body region (subject 1) …............................................62

Figure 3.6 Time history of resultant velocity on each body region (subject 2) ….............................................62

Figure 3.7 Time history of resultant acceleration on each body region (subject 1) ..........................................63

Figure 3.8 Time history of acceleration on each body region (subject 2) …...................................................63

Figure 3.9 Comparison of injury prediction of subject 1 for morphed model, baseline model, and PMHS injury from Subit et al. 2008 .66

Figure 3.10 Comparison of injury prediction of subject 2 for morphed model, baseline model, and PMHS injury from

Subit et al. 2008

Figure 3.11 Trajectories in vehicle coordinate system (Left - subject 3, Right - subject 4) ................................76

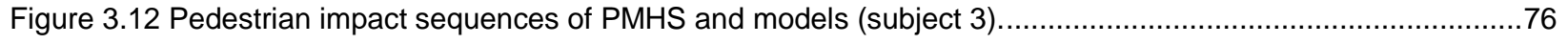

Figure 3.13 Pedestrian impact sequences of PMHS and models (subject 4). …….........................................77

ix 
Figure 3.14 Time history of resultant velocity on each body region (subject 3).....

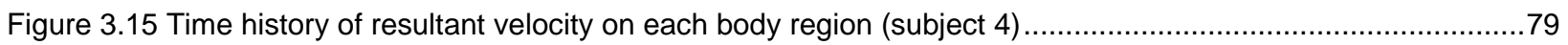

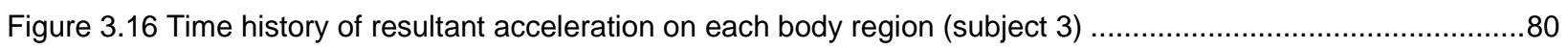

Figure 3.17 Time history of resultant acceleration on each body region (subject 4 ) ...................................... 80

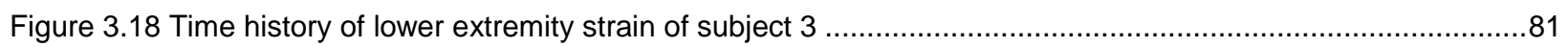

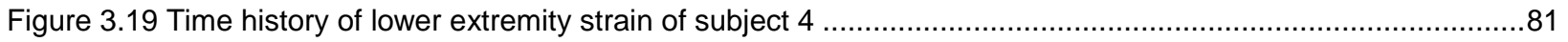

Figure 3.20 Comparison of injury prediction of subject 3 for morphed model and PMHS injury from Subit et al. 200882

Figure 3.21 Comparison of injury prediction of subject 4 for morphed model and PMHS injury from Subit et al. 200883

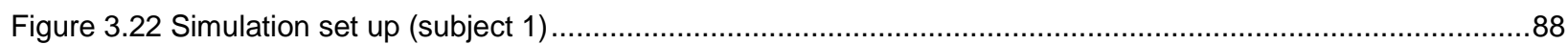

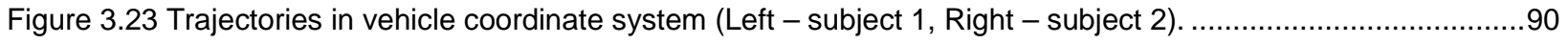

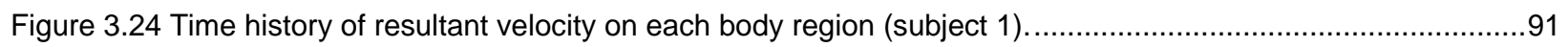

Figure 3.25 Time history of resultant velocity on each body region (subject 2)...........................................91

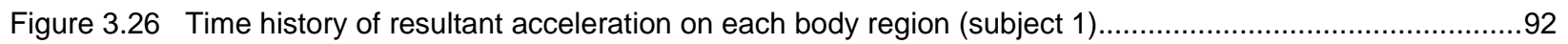

Figure 3.27 Time history of resultant acceleration on each body region (subject 2)...................................92

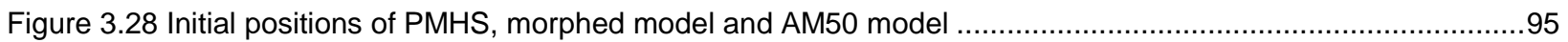

Figure 3.29 Anatomical landmarks and schematic of the centerline cross-section of the mid-size sedan (Subit et al.

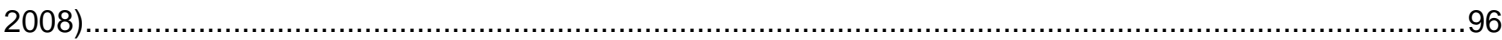

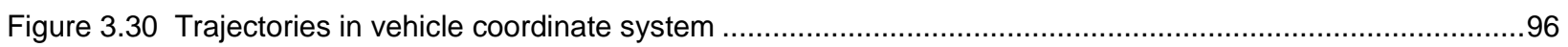

Figure 3.31 Strain gauge locations (PMHS 1) Figure 3.32 Strain gauge locations (PMHS 2) .............................97

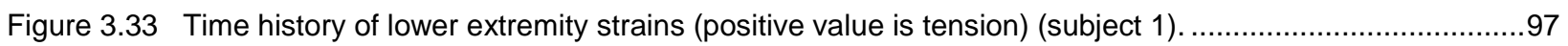

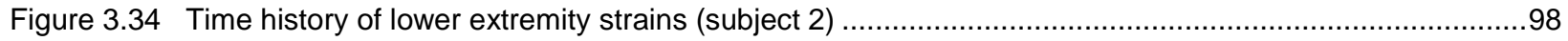

Figure 3.35 Experimental Subjects injury diagrams (left-PMHS1, right-PMHS 2) (Subit et al. 2008) ....................100

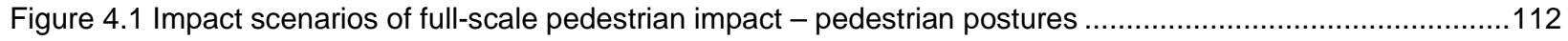

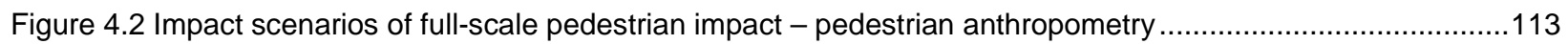

Figure 4.3 Impact scenarios of full-scale pedestrian impact - Pedestrian locations...................................114

Figure 4.4 Impact scenarios of full-scale pedestrian impact - Pedestrian orientations .................................114

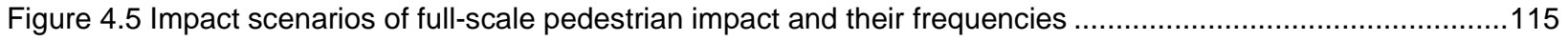

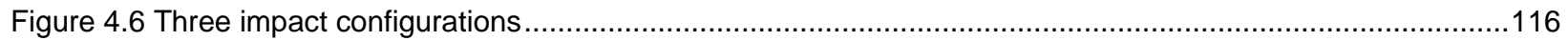

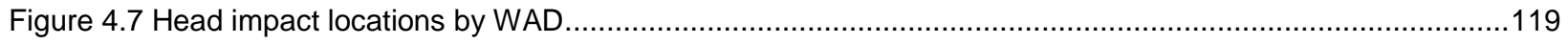


Figure 4.8 Comparison on HIC between impactor tests and full-scale impacts (Left - All impact scenarios; Right -

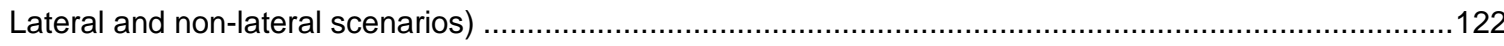

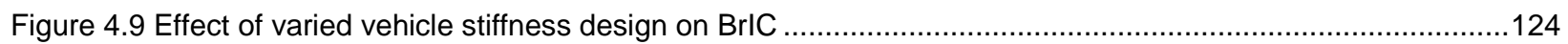

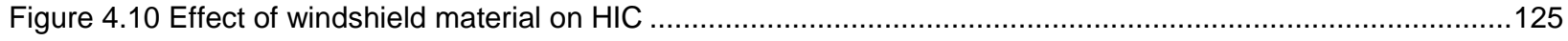

Figure 4.11 Time history of resultant head acceleration of RF85corner (Left - full-scale impact; Right - impactor test)

Figure 4.12 Effect of increasing hood thickness on HIC. 126

Figure 4.13 Time history of head acceleration when varying hood thickness of RF15 (Left - full-scale impact; Right impactor test)

Figure 4.14 Effect of decreasing hood thickness on HIC (Left - All impact scenarios; Right - Lateral impact scenarios)

Figure 4.15 Effect of hood material on HIC (Upper - All impact scenarios; Lower Left - lateral impact scenarios; Lower Right - non-lateral impact scenarios)

Figure 4.16 Time history of head acceleration when varying material stiffness of RF15 (Left - full-scale impact; Right - impactor test). 129

Figure 4.17 Effect of hinge support on HIC (Left - weakening support; Right - strengthening support) 130

Figure 4.18 Time history of head acceleration when varying hood hinge support of RF15 (Left - full-scale impact;

$$
\text { Right - impactor test) }
$$

Figure 4.19 Time history of angular velocity of LF15 (Left - rx; Middle - ry; Right - rz) 133

Figure 4.20 Time history of angular velocity of LF85 (Left - rx; Middle - ry; Right - rz) 140

Figure 4.21 Time history of angular velocity of RF85 (Left - rx; Middle - ry; Right - rz) ....... 141

Figure 4.22 Time history of angular velocity of LF15 (Left - rx; Middle - ry; Right - rz) 141

Figure 4.23 Time history of angular velocity of RF15 (Left - rx; Middle - ry; Right - rz) 141

Figure 4.24 Time history of head impact force of LF85corner (Left - full-scale impact; Right - impactor test) ..........142

Figure 4.25 Time history of head impact force of RF85corner (Left - full-scale impact; Right - impactor test).........142

Figure 4.26 Time history of head impact force of S85corner (Left - full-scale impact; Right - impactor test)...... 143

Figure 4.27 Time history of resultant head acceleration of LF85corner (Left - full-scale impact; Right - impactor test) 
Figure 4.28 Time history of resultant head acceleration of RF85corner (Left - full-scale impact; Right - impactor test)

Figure 4.29 Time history of resultant head acceleration of S85corner (Left - full-scale impact; Right - impactor test)

Figure 4.30 Time history of head impact force when varying material stiffness of LF15 (Left - full-scale impact; Right - impactor test) 145

Figure 4.31 Time history of head acceleration when varying material stiffness of LF15 (Left - full-scale impact; Right impactor test) 145

Figure 4.32 Time history of head impact force when varying material stiffness of RF15 (Left - full-scale impact; Right - impactor test). 146

Figure 4.33 Time history of head acceleration when varying material stiffness of RF15 (Left - full-scale impact; Right - impactor test) 146

Figure 4.34 Time history of head impact force when varying hood thickness of LF15 (Left - full-scale impact; Right impactor test).

Figure 4.35 Time history of head acceleration when varying hood thickness of LF15 (Left - full-scale impact; Right impactor test)

Figure 4.36 Time history of head impact force when varying hood thickness of RF15 (Left - full-scale impact; Right impactor test).

Figure 4.37 Time history of head acceleration when varying hood thickness of RF15 (Left - full-scale impact; Right impactor test) 148

Figure 4.38 Time history of head impact force when varying hood hinge support of LF15 (Left - full-scale impact; Right - impactor test)......

Figure 4.39 Time history of head acceleration when varying hood hinge support of LF15 (Left - full-scale impact; Right - impactor test)

Figure 4.40 Time history of head impact force when varying hood hinge support of RF15 (Left - full-scale impact; Right - impactor test) 150

Figure 4.41 Time history of head acceleration when varying hood hinge support of RF15 (Left - full-scale impact; Right - impactor test) 150

Figure 5.1 Experiments to study the effect of factors 153

Figure 5.2 Selected impact locations 155 
Figure 5.3 Impactor tests with prescribed constant speed

Figure 5.4 HIC of full-scale impacts and impactor tests (Left - before-matching kinematics; Right - after matching kinematics).

Figure 5.5 HIC of full-scale impacts and impactor tests (Left - Non-lateral impact scenarios; Right - Lateral impact scenarios) 158

Figure 5.6 HIC of full-scale impacts and impactor tests (Left - before-matching kinematics; Right - after matching kinematics).

Figure 5.7 HIC of full-scale impacts and impactor tests (Left - Non-lateral impact scenarios; Right - Lateral impact scenarios) 159

Figure 5.8 Vehicle pre-deformation in full-scale impact of impact configuration 2 (Left -Lateral impact scenario; Right Non-lateral impact scenario) 160

Figure 5.9 Errors of each experiment of all 11 cases in impact configuration 2 163

Figure 5.10 Absolute errors of each experiment of all 11 cases in impact configuration 2. 163

Figure 5.11 Errors of each experiment of 6 lateral impact cases in impact configuration 2. 164

Figure 5.12 Absolute errors of each experiment of 6 lateral impact cases in impact configuration 2 165

Figure 5.13 Time history of experiment 2 and 3 in impact scenario LF15 (Left - head impact force; Right - head acceleration) 165

Figure 5.14 Time history of experiment 2 and 3 in impact scenario RF15 (Left - head impact force; Right - head acceleration) 166

Figure 5.15 Errors of each experiment of 5 non-lateral impact cases in impact configuration 2 167

Figure 5.16 Absolute errors of each experiment of 5 non-lateral impact cases in impact configuration 2 167

Figure 5.17 Time history of experiment 2 and 3 in impact scenario LF1590 (Left - head impact force; Right - head acceleration) 168

Figure 5.18 Time history of experiment 2 and 3 in impact scenario RF1590 (Left - head impact force; Right - head acceleration) 168

Figure 5.19 Force-deflection response of steel hood 169

Figure 5.20 Force-deflection response of aluminum hood 170

Figure 6.1 Conceptual design of upper body impactor test 181 


\section{$\underline{\text { List of Tables }}$}

Table 1.1 Distributions of Pedestrian Injury in each Country (AIS2-6) \% (Chen et al. 2009) ................................

Table 2.1 Distributions of Pedestrian Impact by Ages and Countries (AIS1-6) \% (Chen et al. 2009) .......................16

Table 2.2 IHRA Pedestrian Injuries by Body Region and Vehicle Contact Source - All Age Groups; AIS 2-6 (Mizuno. 2003)....... 20

Table 3.1 Injury criteria. .55

Table 3.2 Strain threshold used in the deterministic method. .56

Table 3.3 Model evaluation results using the CORA method. .59

Table 3.4 CORA ratings (Subject 1 ). 60

Table 3.5 CORA ratings (Subject 2)...... .61

Table 3.6 Difference of CORA sub-scores between morphed models and baseline models 61

Table 3.7 Summary of predicted injury for PMHS 1, morphed model (Morphed 1) and baseline model (Baseline 1) using probabilistic method (PM) and deterministic method (DM) (Inconsistent model predictions with PMHS are bolded and underlined). 64

Table 3.8 Summary of predicted injury for PMHS 2, morphed model (Morphed 2) and baseline model (Baseline 2) using probabilistic method (PM) and deterministic method (DM). .65

Table 3.9 Model evaluation results using CORA method (morphed 3, 4) .77

Table 3.10 CORA ratings (Subject 3,4 )

Table 3.11 Summary of predicted injury for PMHS 3 and Morphed 3 using probabilistic (PM) and deterministic method (DM) (Model prediction inconsistent with PMHS are bolded and underlined). .83

Table 3.12 Summary of predicted injury for PMHS 4 and Morphed 4 using probabilistic (PM) and deterministic method (DM) (Model prediction inconsistent with PMHS are bolded and underlined). . .84

Table 3.13 Simulation matrix. 88

Table 3.14 Injury criteria. 89

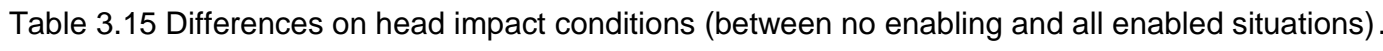
90

Table 3.16 Summary of predicted injury of subject 1, for baseline simulation, simulation of low quality bone and failure all enabled using probabilistic method (PM) .93 
Table 3.17 Summary of predicted injury of subject 2, for baseline simulation, simulation of low quality bone, failure limb enabled, and failure all enabled using probabilistic method (PM) .....

Table 3.18 Detailed anthropometry parameters (errors with respect to PMHS are in bracket). .95

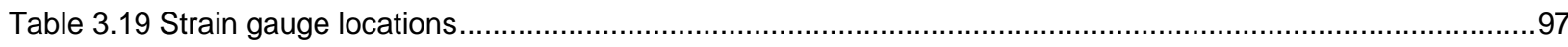

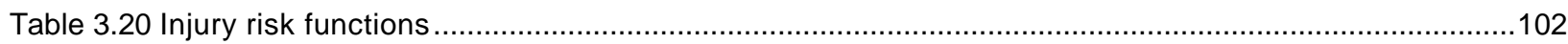

Table 3.21 Summary of predicted injury for PMHS 1 and Morphed 1 using probabilistic (PM) and deterministic method (DM) (Model prediction inconsistent with PMHS are bolded and underlined). 102

Table 3.22 Summary of predicted injury for PMHS 2 and Morphed 2 using probabilistic (PM) and deterministic method (DM) (Model prediction inconsistent with PMHS are bolded and underlined). 103

Table 3.23 Predicted injury risk (morphed model1). 104

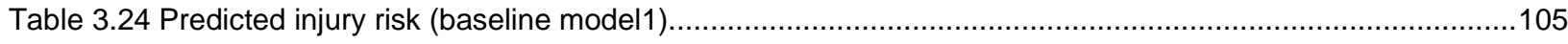

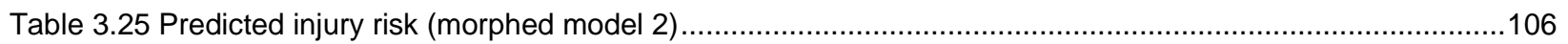

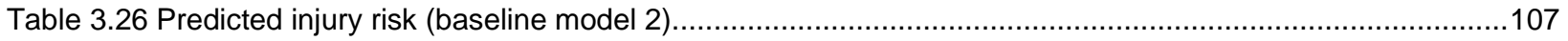

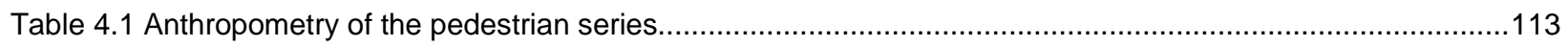

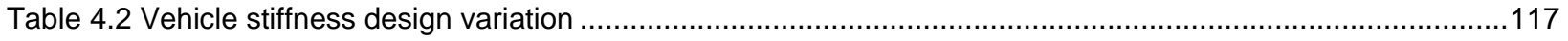

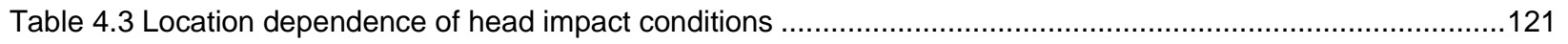

Table 4.4 Head response in full-scale impact and impactor response in sub-system tests...............................137

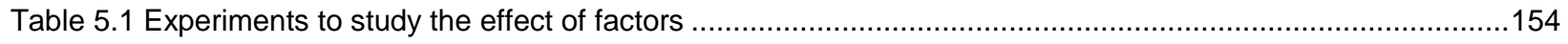

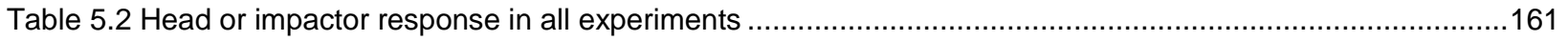

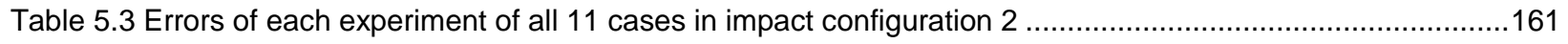

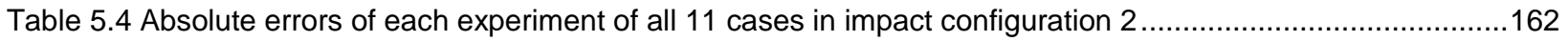

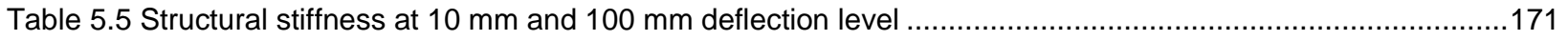




\section{Chapter 1 Introduction}

\subsection{Problem Statement}

According to a WHO report in 2013, over 270,000 pedestrians are killed each year worldwide and account for $22 \%$ of all road traffic fatalities. Pedestrian crashes are more frequent in low-income countries than high-income countries, however, between 2009 and 2012 the number of pedestrian fatalities increased slightly in middle- and high-income countries (IRTAD 2014). In some countries, more than half of those killed on the road are pedestrians (World Health Organization, 2013).

Head injuries are the main cause of death in pedestrian crashes (Hardy 2009; Lau et al. 1998). Non-fatal head injuries can cause long-term medical impairment (Olver et al. 1996). According to the PCDS's distribution of injuries by severity level and frequency, head injury accounts for $80 \%$ of the serious injuries (AIS 5-6), and 35\% of pedestrian moderate injuries (AIS 2-4) (Crandall et al. 2002) (Figure 1.1). According to in-depth accident investigations in the US, Germany, Japan, Australia, and China (Chen et al. 2009) (Table 1.1), among injury severity AIS 2-6, head and lower extremities are the most frequent injury regions.

In a typical pedestrian accident with a passenger car, the pedestrian's lower extremity is accelerated forward and the upper body is rotated and accelerated towards the car. Consequently, the pelvis is generally impacted by the bonnet leading edge, and thorax is impacted by the bonnet top. The head may hit the bonnet or windscreen. 


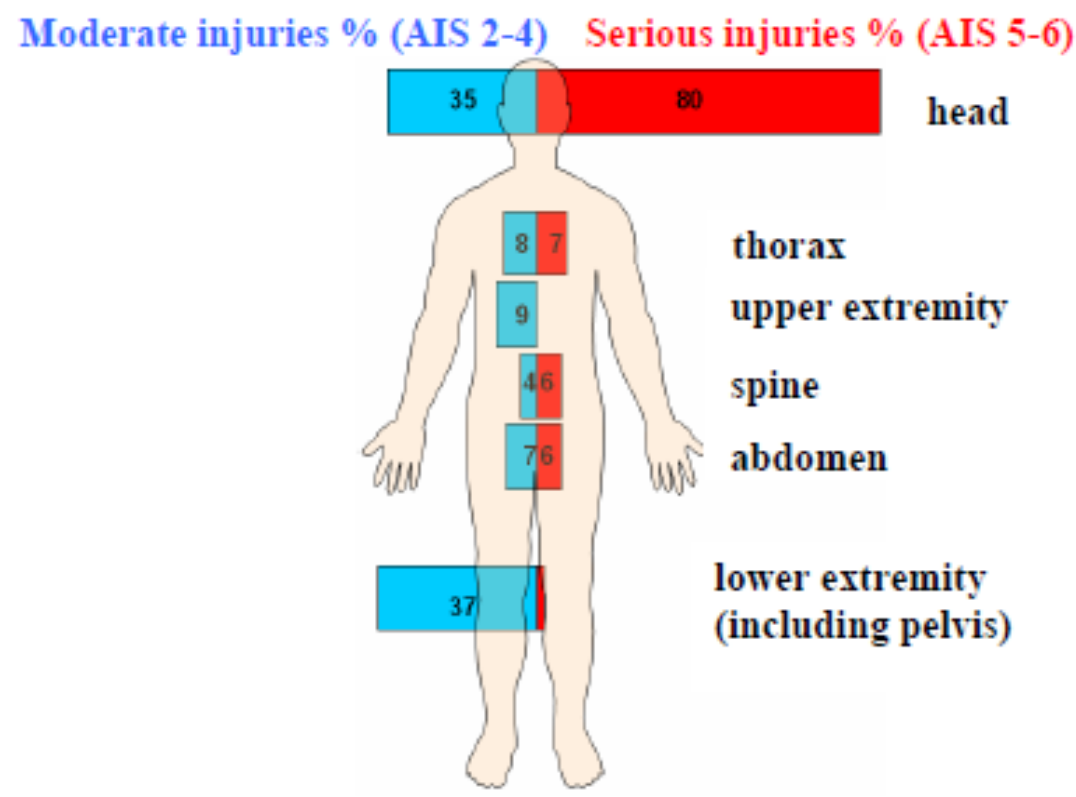

Figure 1.1 Distribution of pedestrian injuries in vehicle-pedestrian accidents (adapted from Crandall et al. 2002)

Table 1.1 Distributions of Pedestrian Injury in each Country (AIS2-6) \% (Chen et al. 2009)

\begin{tabular}{ccccccc}
\hline Body Region & China & IHRA & USA & Germany & Japan & Australia \\
\hline Head & 38.6 & 31.4 & 32.7 & 29.9 & 28.9 & 39.3 \\
Face & 0.7 & 4.2 & 3.7 & 5.2 & 2.2 & 3.7 \\
Neck & 0.2 & 1.4 & 0.0 & 1.7 & 4.7 & 3.1 \\
Chest & 11.6 & 10.3 & 9.4 & 11.7 & 8.6 & 10.4 \\
Abdomen & 5.3 & 5.4 & 7.7 & 3.4 & 4.7 & 4.9 \\
Pelvis & 5.1 & 6.3 & 5.3 & 7.9 & 4.4 & 4.9 \\
Arms & 9.9 & 8.2 & 7.9 & 8.2 & 9.2 & 8.0 \\
Legs & 27.2 & 32.6 & 33.3 & 31.6 & 37.2 & 25.8 \\
Unknown & 1.4 & 0.2 & 0.0 & 0.4 & 0.0 & 0.0 \\
\hline
\end{tabular}

To combat this substantial public health problem, pedestrian regulations were made to assess the vehicle aggressiveness. In the testing environment, the full-scale dummy test is an obvious option to reproduce pedestrian head impact, but it is not appropriate for regulatory purposes because in real accidents, there are numerous potential locations the pedestrian's head could impact. To cover all possible accident situations, numerous dummy tests would be needed, 
but this is clearly impossible. Therefore, the simplified sub-system test procedure was proposed and used to distinguish vehicle stiffness by firing the headform into the vehicle surface to reproduce the head impact. Compared to full-scale dummy tests, this test methodology is advantageous because it can test all potential impact locations with better repeatability and much lower costs.

The input for the head impact conditions for the test procedure, including the impact location, impact speed, angle, and effective mass cannot be obtained without the full-scale impact system. Therefore, three working groups, EEVC, ISO, and IHRA were set up to generate the head impact conditions for the input of the pedestrian sub-system test procedure using PMHS tests data and multi-body simulations.

The accuracy of the current test methodology to represent the full-scale impact has long been criticized but has not been thoroughly investigated; nonetheless, for the last decade, it has been the only test procedure for head protection for pedestrian regulation and the NCAP. An obvious limitation of this test methodology is the absence of the head-neck interaction, vehicle pre-deformation, and head rotational kinematics. Previous PMHS tests (Kerrigan et al. 2008) observed higher HIC although with lower head impact speed than the impactor test, which suggested the potential difference between head response and impactor response. This makes the ability of the component test to represent the real full-scale impact still questionable, and could potentially cause a big problem because vehicle design that is optimized to the sub-system test may not necessarily provide the best protection in real pedestrian accidents.

Previous efforts have focused on optimizing vehicle design towards the component procedure (Kerkeling et al. 2005; Liu et al. 2009), however vehicles designed towards the fullscale impact have not been well studied. This lack of research is partly because previously 
existing methods and tools are not very appropriate for this purpose. The physical test is not appropriate for this purpose because testing the various vehicle designs requires many tests at a huge cost. The PMHS tests cannot be repeated to allow for varying one factor at a time to study its effect, and different intrinsic properties among cadavers do not make it feasible for parametric study. Unfortunately, for the dummy tests only a very limited anthropometry was available. The multi-body modeling cannot accurately model the vehicle pre-deformation, and lacks accuracy on biofidelity, head-neck interaction, and head contact geometry.

Recently, the Pedestrian Finite Element Models (PFEM) have been developed, validated, and widely used. These models, with refined geometry, advanced material laws, and detailed insight, provided better tools to predict pedestrian kinematics and injury risk. Similarly, the FE vehicle models provided better modeling on vehicle contact and deformation. These methods and models provided conditions to verify the accuracy of current test methodology to represent the full-scale impact by further investigating the relationship between the impactor responses of the component test with the head response of the full-scale pedestrian impact, and the effect of those factors such as the head-neck interaction, vehicle pre-deformation, and head rotational kinematics on the head response.

\subsection{Objective}

The overall goal of this dissertation is to study the relationship between the impactor responses of the sub-system test with the human head response in the full-scale pedestrian impact. To achieve this goal, specifically, this dissertation includes three steps: 
The first step is the PFEM biofidelity evaluation. This is achieved by evaluating the biofidelity of the THUMS pedestrian model, and a study of enabling tissue failure to verify the insensitivity on the pedestrian global response and head impact conditions.

The second step is to compare the impactor response in the sub-system test and head response in full-scale impact under diverse impact scenarios, and test the hypothesis that vehicle design variations have different influences on the impactor response and head response.

The last step is to explore the reasons for the second step. That is, to study the effect of those factors that may differ head response in the sub-system test from the full-scale impact, including the head kinematics, head-neck interaction, vehicle pre-deformation, and head initial rotation.

\subsection{Hypothesis and Specific Aims}

The main hypotheses are the current sub-system test procedure cannot accurately capture the human head impact response in the full-scale impact, and while this vehicle design variations have different impacts on the impactor response and head response. Before this hypothesis is tested, the THUMS PFEM biofidelity will be evaluated, and the effect of enabling tissue failure on model biofidelity evaluation will be verified. Finally, the reasons for the results of the hypothesis testing will be examined. In total, there are six hypotheses examined in this dissertation. The specific aims and hypotheses are presented below.

Hypothesis 1 - THUMS PFEM is deemed biofidelic against PMHS response in full-scale vehicle-pedestrian tests.

Hypothesis 2 - Pedestrian global response is not significantly sensitive to the tissue failure during the impact. 
Hypothesis 3 - Current NCAP test procedure cannot accurately capture the head impact response at $40 \mathrm{~km} / \mathrm{h}$ vehicle impact speed under diverse impact scenarios.

Hypothesis 4 - Vehicle design variations have different influences on the impactor response and head response.

Hypothesis 5 - The primary reason for the current NCAP procedure underestimating the head impact response at $40 \mathrm{~km} / \mathrm{h}$ vehicle impact speed is the lower input head impact conditions.

Hypothesis 6 - Vehicle pre-deformation caused by torso impact can fundamentally affect the head response, and cars that perform better in NCAP does not necessarily mean they obtain lower HIC in full-scale impact. 


\section{Chapter 2 Literature Survey}

In order to complete the main objective of this study - to study the relationship between the impactor responses of the sub-system test with the human head response in full-scale pedestrian impact, a literature survey was conducted to review the current knowledge of the phenomenon under study - the car-to-pedestrian accidents, head injury biomechanics, and the method and tools to study the pedestrian head injury.

First, a short review of relevant human head anatomy, pedestrian head injury type, mechanism, and injury criteria are provided. Second, the methods for evaluating the pedestrian head injuries are presented, including physical tests and mathematical models.

A summary of the literature survey is provided in this chapter.

\subsection{Pedestrian Head Injury Biomechanics}

\subsubsection{Head Anatomy Review}

In order to discuss the potential head injuries that result from pedestrian accidents, it is important to first understand the anatomy of the head. A human head can be divided into multiple structures, and mainly includes the scalp, skull, meninges, brain, face, teeth, and other structures, and Figure 2.1shows the main head structures. 


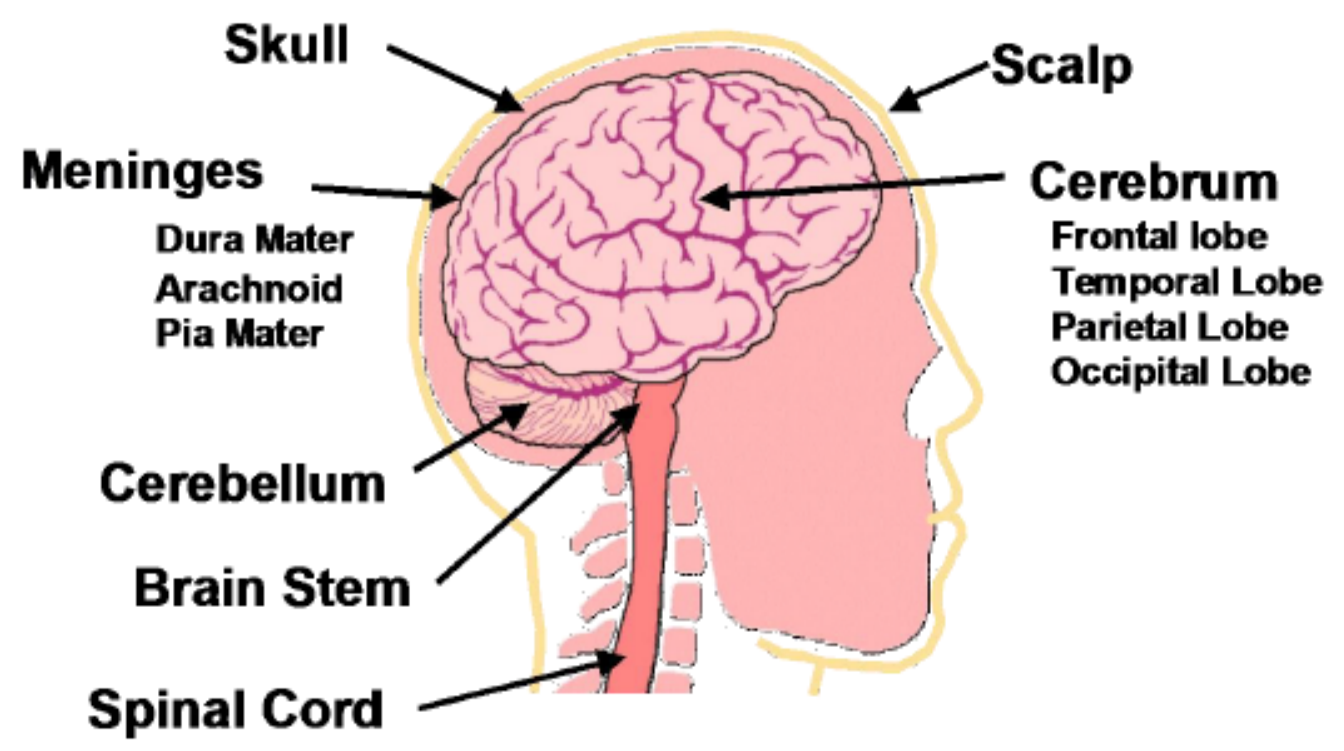

Figure 2.1 Main head structures (Arregui et al. 2006)

The scalp skin covers the head and is a soft tissue layer that serves as the first defense against impact trauma. As the outermost layer, it is about 5-7 mm thick, and is made up of 5 layers including skin, connective tissue, aponeurosis, lose connective tissue, and pericranium.

The skull is the body's most complex bony structure because it encloses and protects (1) the irregularly shaped brain, (2) the organs of special senses (sight, hearing, taste, and smell), and (3) the openings of the digestive and respiratory tracts. An adult's skull is a structure formed by bones fused together and associated suture lines. It is formed by a total of 22 bones that are classified as either cranial (8) or facial bones (14) (Figure 2.2). The cranium has a domelike roof, the calvaria, and a floor or cranial base consisting of the ethmoid bone and parts of the occipital and temporal bones. The facial skeleton consists of bones surrounding the mouth and nose and contributing to the orbits. 


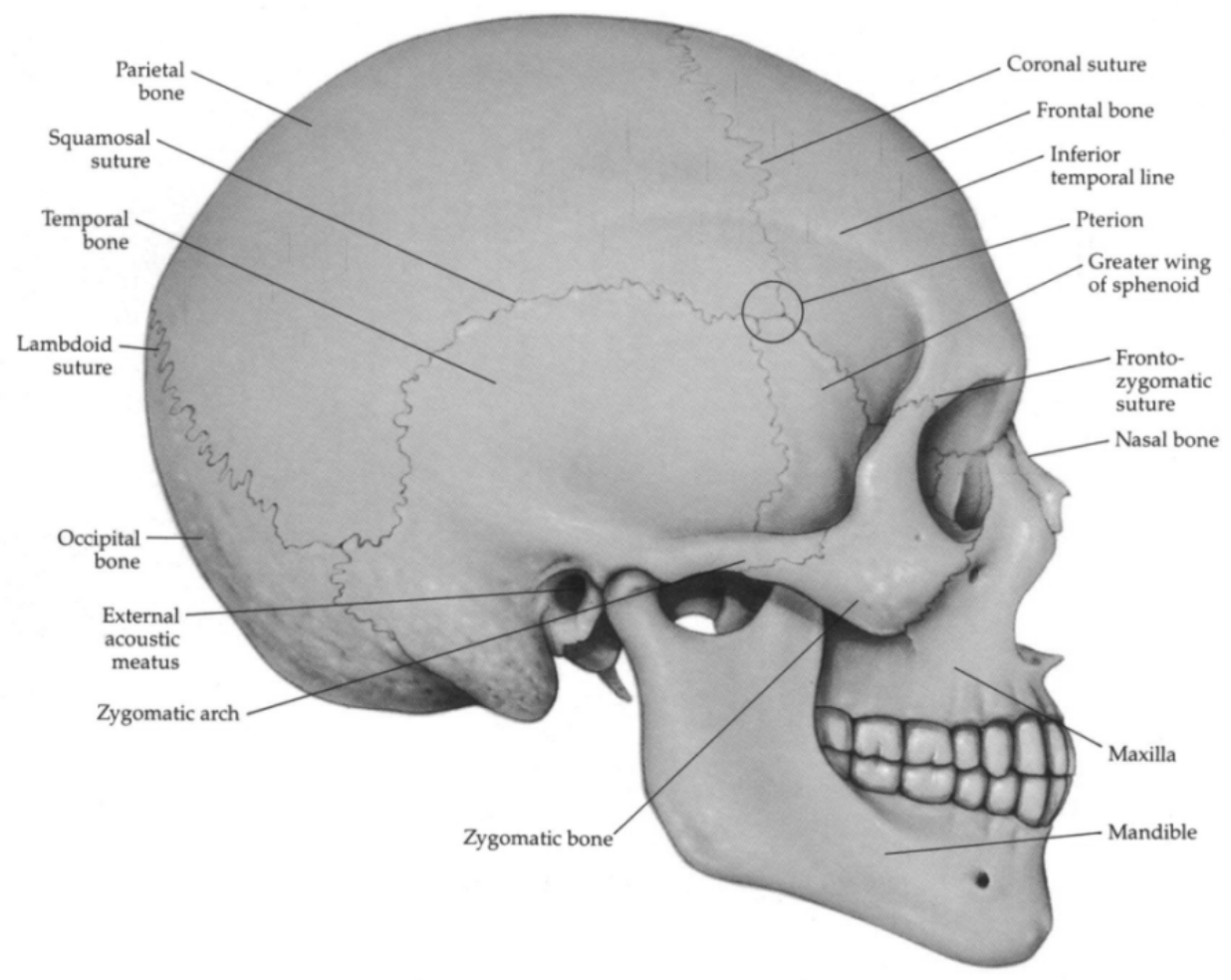

Figure 2.2 Lateral view of the human skull

Meninges are the intracranial soft tissue layers that lie internal to the skull bones and protect and support the central nervous system (CNS) (Figure 2.3). The meninges are primarily made up of connective tissue, and serve to isolate the CNS from the surrounding bone structure. They also form part of the blood vessel walls and nerve sheaths as they traverse the skull boundary, as well as contain cerebrospinal fluid. The meninges consist of three primary layers. Starting from the skull bone and working in, they are the dura mater, arachnoid and pia mater. The dura mater is a tough, double layered membrane that surrounds the brain. The outer periosteal layer is the inner periosteum covering of the cranium. The inner meningeal layer is the outermost brain covering and extends inferiorly to surround the spinal cord as the dural sheath. Separating the dura mater from the inferior arachnoid mater is a narrow cavity called the 
subdural space. The arachnoid, for short, is a loosely embedded membrane of the meninges. Between the arachnoid and pia mater is another, much wider cavity labeled the subarachnoid space. This space is spanned by arachnoid extensions that secure the arachnoid to the underlying pia mater. The subarachnoid space is filled with cerebrospinal fluid (CSF) and contains the largest blood vessels supplying the brain. The CSF provides some nutrients to the brain and provides resistance to mechanical shock. The innermost intracranial soft tissue layer encapsulating the brain is the pia mater, a delicate connective tissue abundant with minute blood vessels.

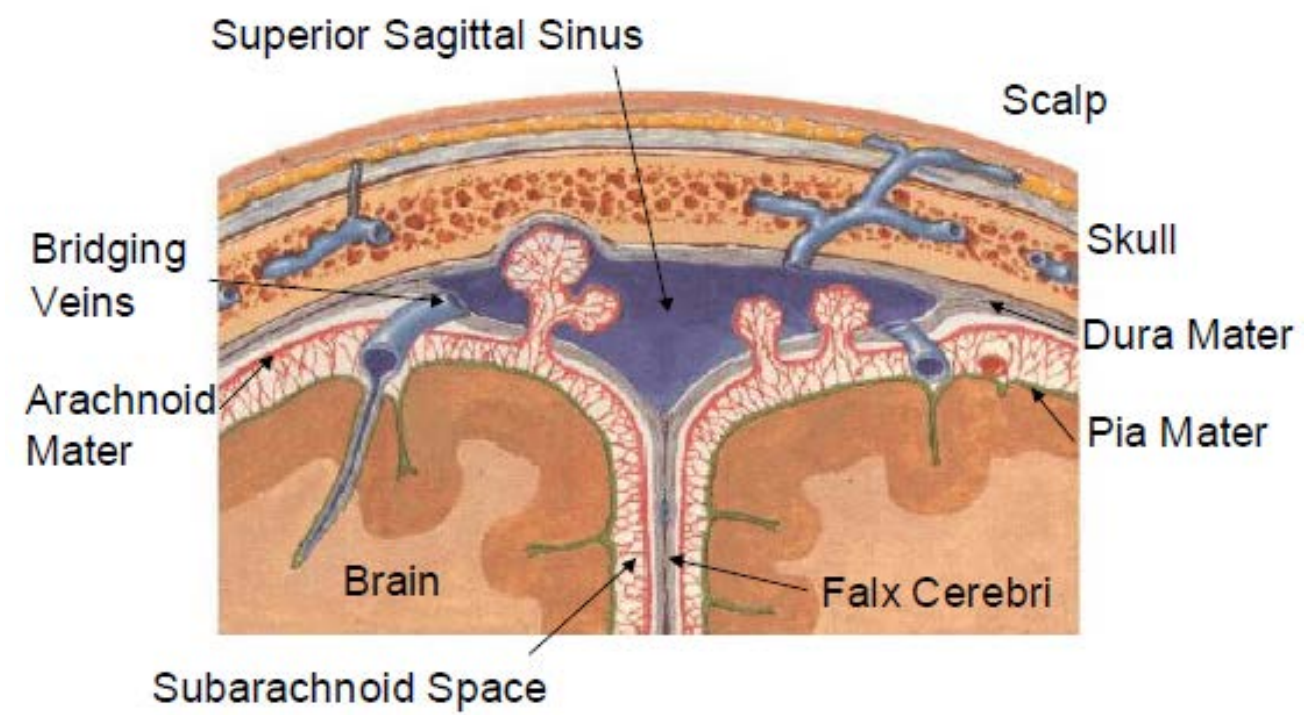

Figure 2.3 The cranial meninges (Arregui et al. 2006)

Finally, the central nervous system (Figure 2.4) consisting of the brain and spinal cord is located at the center of the head. The brain is enclosed within the skull, and it is responsible for controlling our body and providing individuals with their unique behaviors. The nervous tissue of the brain is quite soft and delicate, and even slight pressures can irreversibly injure the contained neurons. Structurally, the brain is made up of five components: cerebrum, cerebellum, 
midbrain, pons and medulla oblongata. In addition, it has 4 ventricles, 3 membranes, 2 glands, 12 cranial nerve pairs, and the cranial arteries and veins.

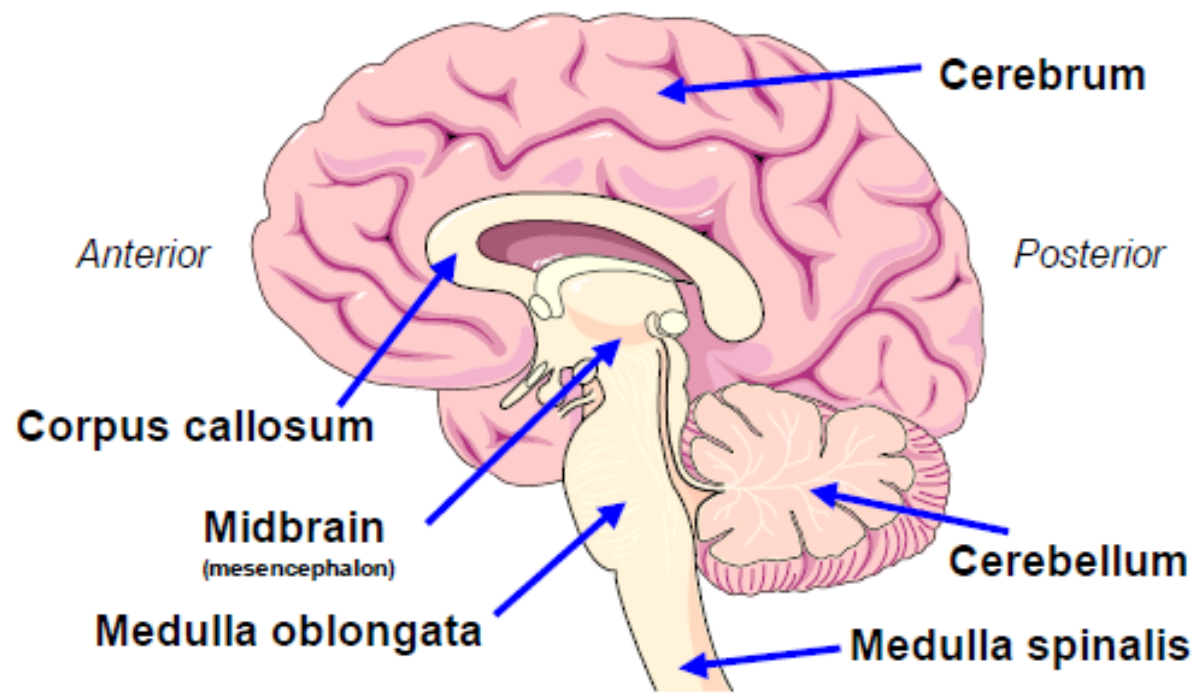

Figure 2.4 The central nervous system (Arregui et al. 2006)

\subsubsection{Pedestrian Accident Conditions}

Since pedestrian head response depends highly on the accident conditions (Chen et al. 2015; Watanabe et al. 2012; Han et al. 2012; Elliote et al. 2012; Subit et al. 2008), including multiple factors such as vehicle impact speed, vehicle type and geometry, human anthropometry, pedestrian initial position and direction to the vehicle, and their postures, the distribution of these accident conditions in field data and their effects on pedestrian response and injury are summarized here.

\section{Vehicle Impact Speed}

In-depth accident investigations in 5 countries (USA, Germany, Japan, Australia, and China) has shown that more than $60 \%$ of pedestrian accidents with an AIS $2+$ injury were under $40 \mathrm{~km} / \mathrm{h}$ vehicle impact speed (Chen et al. 2009; Mizuno 2005) (Figure 2.5). In this dataset, the peak of the frequency of vehicle impact speed for pedestrian accidents with AIS 2+ injury was 
found to be around $40 \mathrm{~km} / \mathrm{h}$, and when the speed is higher than $40 \mathrm{~km} / \mathrm{h}$, the frequency drastically decreases (Figure 2.6). The pedestrian AIS 2+ injury risk was found to be around $70 \%$ at 40 $\mathrm{km} / \mathrm{h}$ vehicle impact speed.

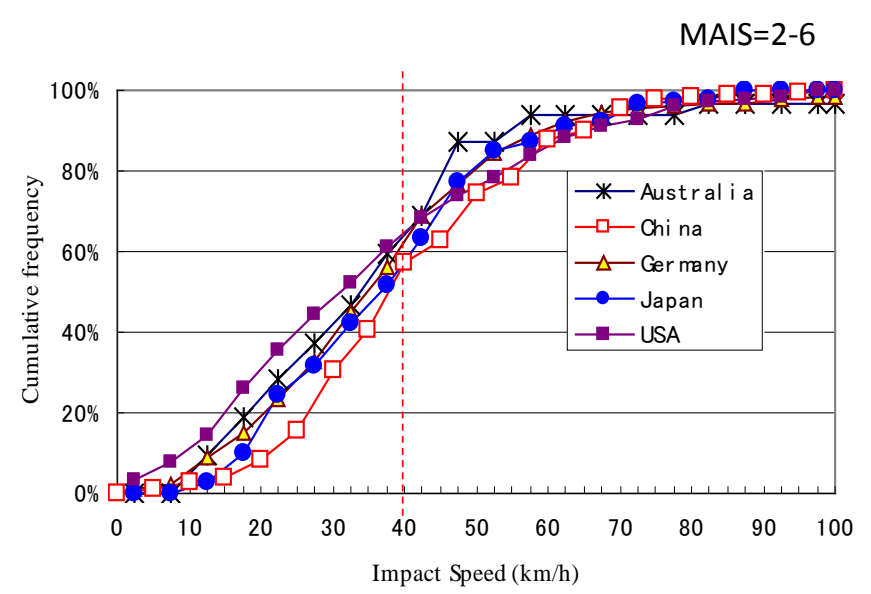

Figure 2.5 Cumulative vehicle impact speed distribution (Chen et al. 2009)

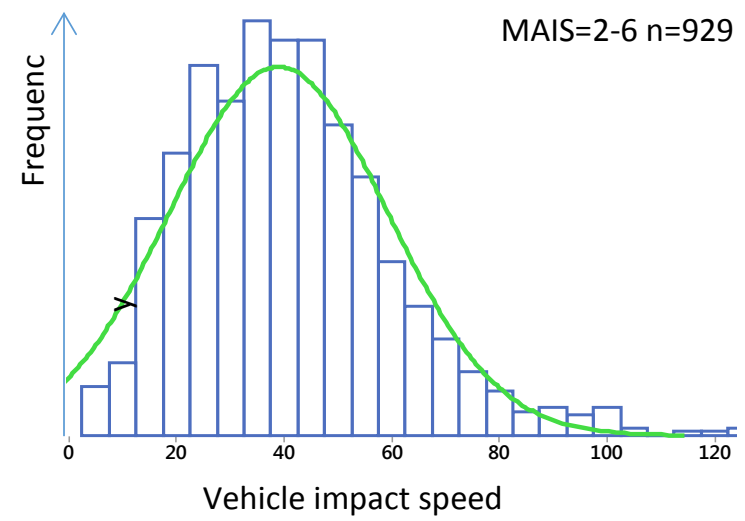

Figure 2.6 Frequency of vehicle impact speed in pedestrian accidents (Chen et al. 2009)

Rosén et al. (2009) queried the German In-Depth Accident Study (GIDAS) for pedestrian accidents during the years 1999-2007. In 490 cases of pedestrians hit by the front of a passenger car with an assessed impact speed, around $80 \%$ of pedestrian accidents with injury (AIS 1+) were under $40 \mathrm{~km} / \mathrm{h}$ vehicle impact speed (Figure 2.7). A strong dependence of pedestrian 
fatality risk on impact speed is present, with the risk at $50 \mathrm{~km} / \mathrm{h}$ being more than twice as high as the risk at $40 \mathrm{~km} / \mathrm{h}$ and more than five times higher than the risk at $30 \mathrm{~km} / \mathrm{h}$ (Figure 2.8).

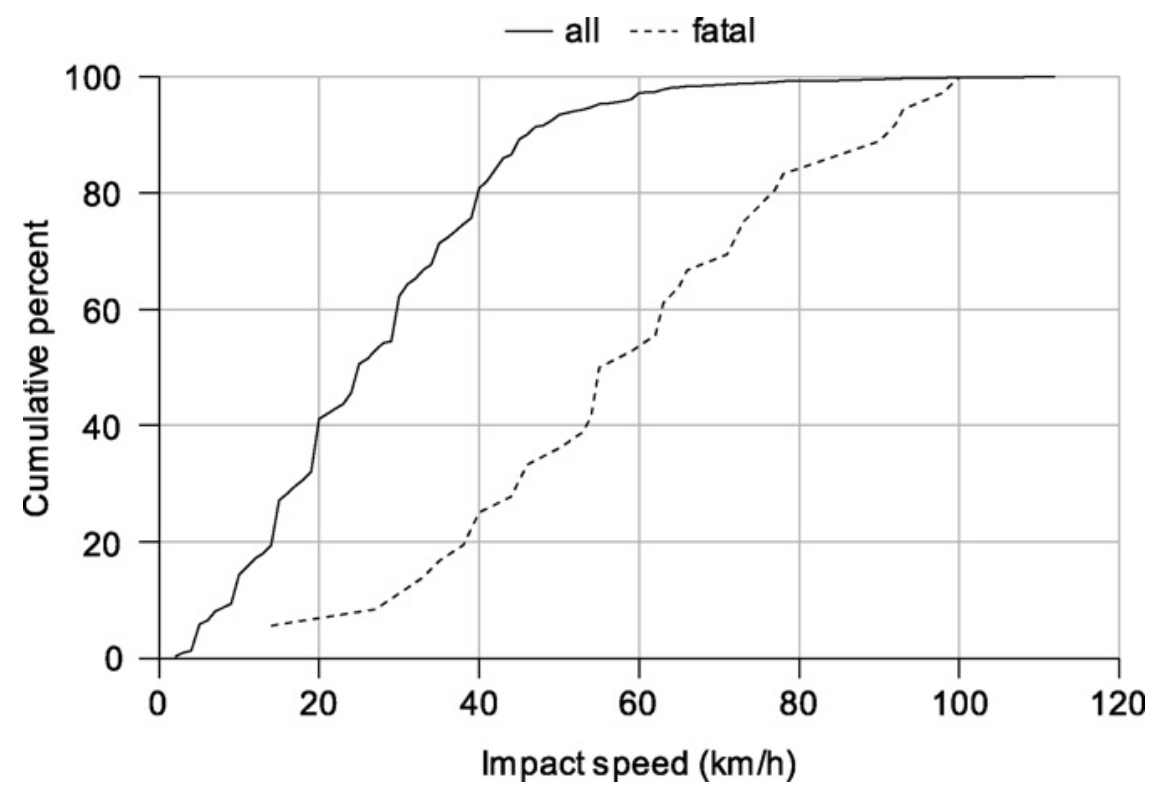

Figure 2.7 Frequency of vehicle impact speed in pedestrian accidents (Rosén et al. 2009)

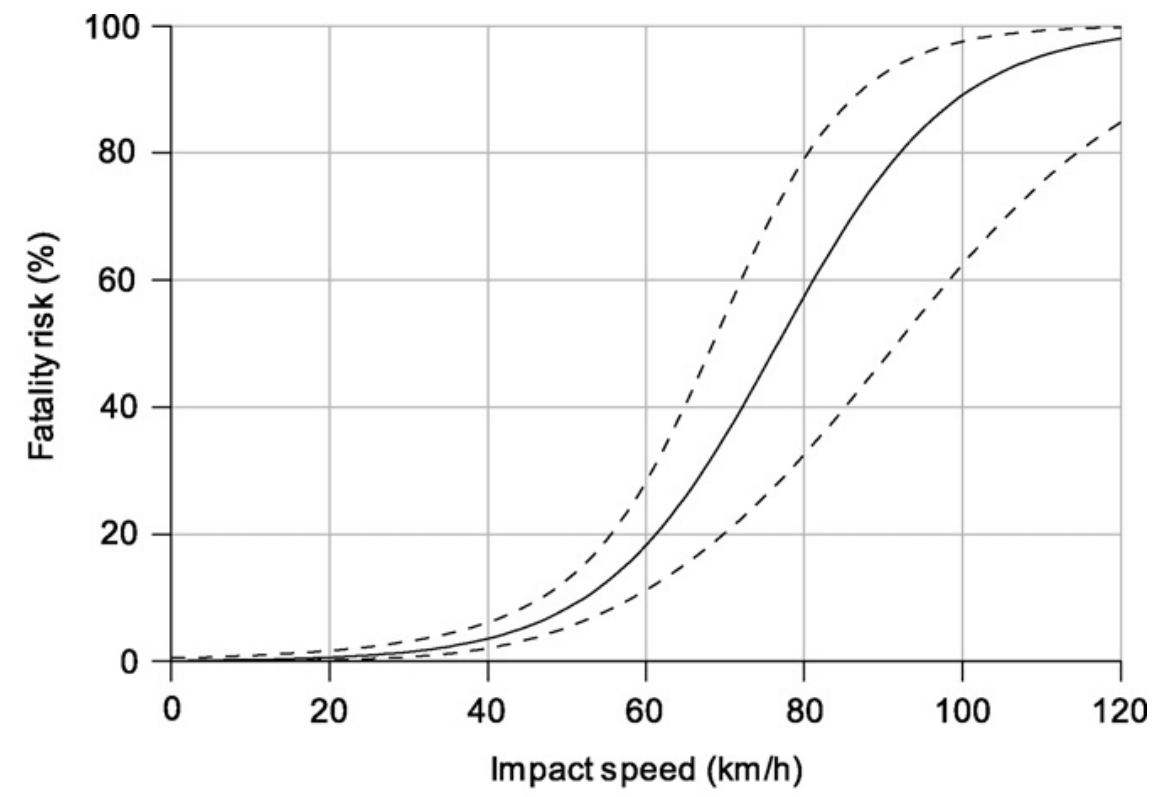

Figure 2.8 The fatality risk as a function of impact speed for adult pedestrians hit by the front of a passenger car. The dotted curves show approximate 95\% confidence limits. (Rosén et al. 2009) 
In the other in-depth accident study by Hamdane et al. (2014), based on the reconstruction of 100 real-world crashes in France and Australia involving a pedestrian and vehicle, 95\% of the vehicle travel speeds are distributed along a wide range from 20 to $60 \mathrm{~km} / \mathrm{h}$, with a peak frequency around $40 \mathrm{~km} / \mathrm{h}$, while the average vehicle impact speed is $32 \mathrm{~km} / \mathrm{h}$.

Watanabe et al. (2012) and Han et al. (2012) conducted simulation studies on the effect of vehicle impact speed on pedestrian kinematics and injury risk using Finite element vehicle model and THUMS PFEM. The collision speed was found to be one of the primary factors of pedestrian severe injuries in the simulation results, and a significant reduction of all injuries can be achieved for all vehicle types when the vehicle impact velocity is less than $30 \mathrm{~km} / \mathrm{h}$.

\section{Vehicle design}

There are hundreds of vehicle types which can be potentially involved in a pedestrian accident, and significant variations were found even within those types (Simms and Wood 2009). Previous studies showed that $66-82 \%$ of pedestrians were hit by passenger cars in Europe (Hardy et al. 2007). In the US, passenger cars are responsible for $68 \%$ of pedestrian injuries and $47 \%$ of pedestrian fatalities, while light trucks are responsible for $28 \%$ of pedestrian injuries and $41 \%$ of fatalities (Untaroiu 2005). In Japan, the ITARDA data in 2009 (Matsui et al. 2013) showed that in fatal accidents, the sedan was the most frequent source (41\%), light passenger car was the second most frequent (26\%), light cargo van was the third (16\%) followed by the minivan (13\%) and box van (4\%).

Accident data showed various vehicle types caused pedestrian injury distribution (Roudsari et al. 2005; Longhitano 2005; Lefler et al. 2004; Ballesteros et al. 2004). Roudsari et al. (2005) found chest and abdomen injuries were more common in pedestrians struck by LTVs. Ballesteros et al. (2004) revealed that compared to passenger vehicles, SUVs and pick-up trucks 
resulted in a higher percentage of injuries to the lower extremities above the knee. Longhitano (2005) used the PCDS data to compare pedestrian injury distribution of light trucks with those of passenger car. It was found that light truck vehicles caused $43 \%$ of AIS 3+ injuries to the lower extremities and $81 \%$ of AIS $3+$ injuries to the head and neck compared to $39 \%$ of injuries to the lower extremities and $71 \%$ of injuries to the head and neck caused by passenger cars.

Also, simulation studies (Watanabe et al. 2012; Han et al. 2012) using FE human models (THUMS) and detailed FE vehicle models showed different pedestrian kinematics and injury risk caused by vehicle types. Han et al. (2012) found vehicle designs with a short front-end and a wide windshield area can protect pedestrians from fatalities. Using parametric FE vehicle front models and pedestrian lower extremity models, Nie and Zhou (2015) verified that recent passenger cars with geometrical changes of front-end design after regulatory efforts can reduce pedestrian lower extremity injury risk.

\section{Pedestrian types, pre-impact location, direction, and posture}

In-depth accident investigations in 5 countries (USA, Germany, Japan, Australia, and China) showed that the 21-60 year old age group has the highest frequency of accident involvement (Chen et al. 2009; Mizuno 2005) (Table 2.1). In terms of sex distribution in the US in $2003,69 \%$ of pedestrian killed and $61 \%$ of pedestrians injured were male (Untaroiu 2005). While in the PCDS dataset in 2001 (Chidester et al 2001), males and females were almost evenly distributed, with a total of 266 males (51\%) and 255 females (49\%). Interestingly, the in-depth pedestrian accident data in China (Chen et al. 2009) also showed higher male injured (57.9\%) than female (42.1\%). Those accident data in 5 countries showed higher injury severity of older pedestrians at a given speed (Chen et al. 2009; Mizuno 2005). Similarly, Niebuhr et al. (2016) queried the GIDAS database for frontal car-to-pedestrian accidents for the years 1999-2013 and 
found 1426 cases, and they verified that older people compared with younger people have a higher MAIS 3+ injury risk at all collision speeds.

Table 2.1 Distributions of Pedestrian Impact by Ages and Countries (AIS1-6) \% (Chen et al. 2009)

\begin{tabular}{ccccccc}
\hline & China & IHRA & USA & Germany & Japan & Australia \\
\hline $0-5$ & 2 & 7.3 & 4 & 9 & 9 & 5 \\
$6-10$ & 4 & 14.1 & 11 & 15 & 20 & 14 \\
$11-15$ & 1.5 & 9.7 & 11 & 10 & 5 & 14 \\
$16-20$ & 6 & 6.6 & 7 & 7 & 3 & 6 \\
$21-60$ & 66.3 & 41.1 & 51 & 36 & 39 & 29 \\
$60+$ & 20.2 & 21.3 & 16 & 23 & 23 & 32 \\
\hline
\end{tabular}

An accident analysis in Europe (Hardy 2009) showed 60-77\% pedestrian were struck by the vehicle front. Similarly, in 2003, NHTSA estimated that $85 \%$ of pedestrians killed, and $80 \%$ of pedestrians injured were struck by the vehicle front end (Untaroiu 2005). Based on the reconstruction of 100 real-world crashes involving a pedestrian and vehicle in France and Australia (Hamdane et al. 2014), the pre-impact location of about $42 \%$ pedestrians are at the center (75\% of the vehicle width). In a volunteer test study (Soni et al. 2013), in $60 \%$ of cases, the pre-impact locations of younger adults were between the center and far-side the vehicle and $60 \%$ of elderly were in the middle of the vehicle.

The majority of pedestrians were struck laterally, and the percentage of pedestrians struck in this manner varied between 65\% (Hardy 2009) and 89\% (Simms and Wood 2009). In the APROSYS European In-Depth Pedestrian Database (Neal-Sturgess et al. 2007), 89\% of the pedestrians were hit on either the right (41\%) or left side (48\%). In the PCDS dataset in US (Chidester et al. 2001), 68\% of the pedestrians struck were orientated with their side to the striking vehicles, with $17 \%$ facing the vehicle and $10 \%$ facing away. In examining the leg 
orientation, $63 \%$ of the pedestrian had one leg forward and apart from the other leg at impact (Chidester et al. 2001).

These factors, including the initial direction, location, anthropometry, and pedestrian posture, were found to strongly affect the pedestrian response on the global kinematics and kinetics in the simulation studies and physical tests (Chen et al. 2015; Li et al. 2015; Soni et al. 2013; Elliott et al. 2012; Subit et al. 2008; Simms et al. 2006).

\subsubsection{Pedestrian Head Injury Biomechanics}

Biomechanics is an interdisciplinary subject in which the mechanics connect engineering to medical science (Cowin, 1989; Fung, 1993; Nahum and Melvin, 2001). From a medical point of view, the mechanics of tissue and organ injuries in vehicle traffic accidents is discussed in terms of treatment, healing, repair, and rehabilitation. From an engineering point of view, the mechanics of human-body injuries in vehicle traffic accidents is discussed in terms of causes of the injuries and the need of engineering designs to avoid accidental injuries. This section focuses on reviewing the pedestrian head injury biomechanics, including accidental head injury and their causes, pedestrian head kinematics, head injury mechanism, and injury criteria.

$\underline{\text { Head injury and their causes }}$

Among life-threatening head injuries in pedestrians, brain (intracranial) injuries are most common, followed by skull fractures (Harruff et al. 1998, Arregui-Dalmases et al. 2010, Leijdesdorff et al. 2014). Of 154 pedestrians with severe traumatic brain injury (sTBI, AIS 3+), 337 injuries were intracranial (89\% cerebrum, 64\% hemorrhage, 52\% contusion, multiple injuries per pedestrian possible) and 149 were skull fractures (Leijdesdorff et al. 2014). In 217 fatally injured pedestrians, 228 intracranial injuries were observed, of which, 120 were cerebral 
contusions / lacerations, 42 were brainstem / midbrain contusions / lacerations and 66 were subdural haematomae, and 150 skull fractures (Harruff et al. 1998).

Mizuno and Kajzer (2000) examined more than 100 pedestrian accidents of the police and in-depth accident databases of ITARDA (Institute for Traffic Accident Research and Data Analysis) in Japan. Figure 2.9 shows the distribution of head injuries classified by injury causes, type and severity. Brain injury is a main cause of the severe head injuries (AIS 3-6). On the other hand, for the minor injuries (AIS 1, 2), the percentages of bruises and lacerations are high. Especially, in contact with the windscreen, the pedestrian is likely to sustain bruises and lacerations unless impact locations are near the windscreen frame. Skull fractures occur frequently when a pedestrian's head contacts with a stiff part such as an A pillar, windscreen frame or the ground. In accidents with bonnet-type cars, the windscreen frame and A pillar were found to have a high potential to cause severe head injury (17\%) followed by the hood (11\%) and the fender (6\%). For minor injuries, the windscreen showed the highest frequency (32\%) among the injury causes of all vehicle parts. 


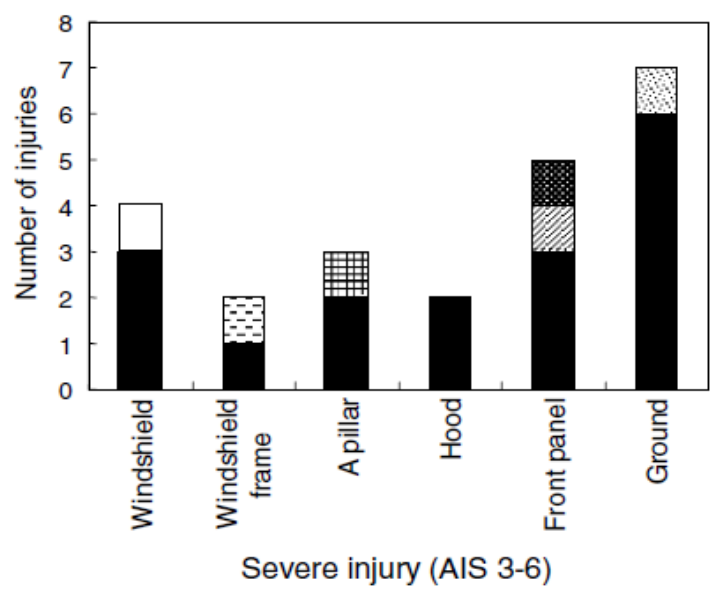

\begin{tabular}{|l|}
\hline Others \\
/Unknown \\
Concussion \\
Abrasion \\
Nerve injury \\
Laceration \\
B Bruise \\
S Skull fracture \\
B Bessel injury \\
B Cerebellum stem injury \\
injury \\
Brain injury
\end{tabular}

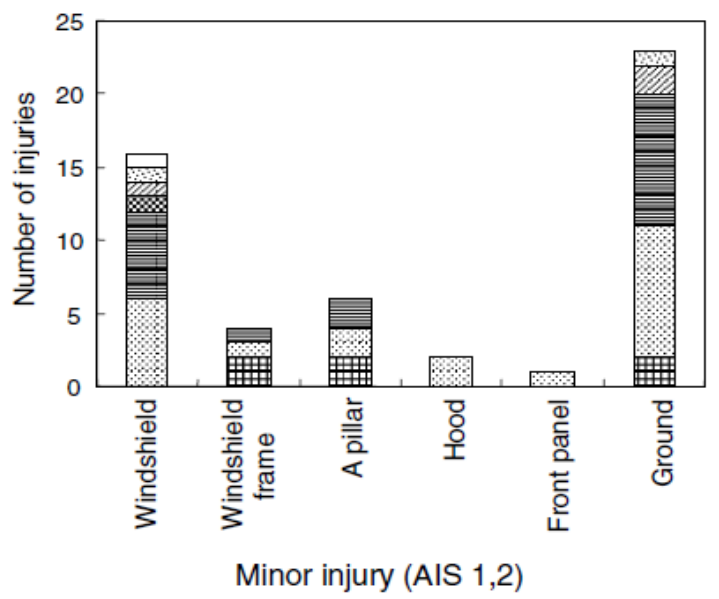

Figure 2.9 Head injury types and injury causes (Mizuno and Kajzer, 2000)

According to the in-depth accident investigation in IHRA countries (Mizuno 2003), the windshield and hood surface are the main injury resources for pedestrian head injury (Table 2.2). Similarly, an accident investigation in China with 200 accident cases (Chen et al. 2009), the windshield and bonnet surface were found to be the leading sources for pedestrian head injury. 
Table 2.2 IHRA Pedestrian Injuries by Body Region and Vehicle Contact Source - All Age Groups; AIS 2-6 (Mizuno. 2003)

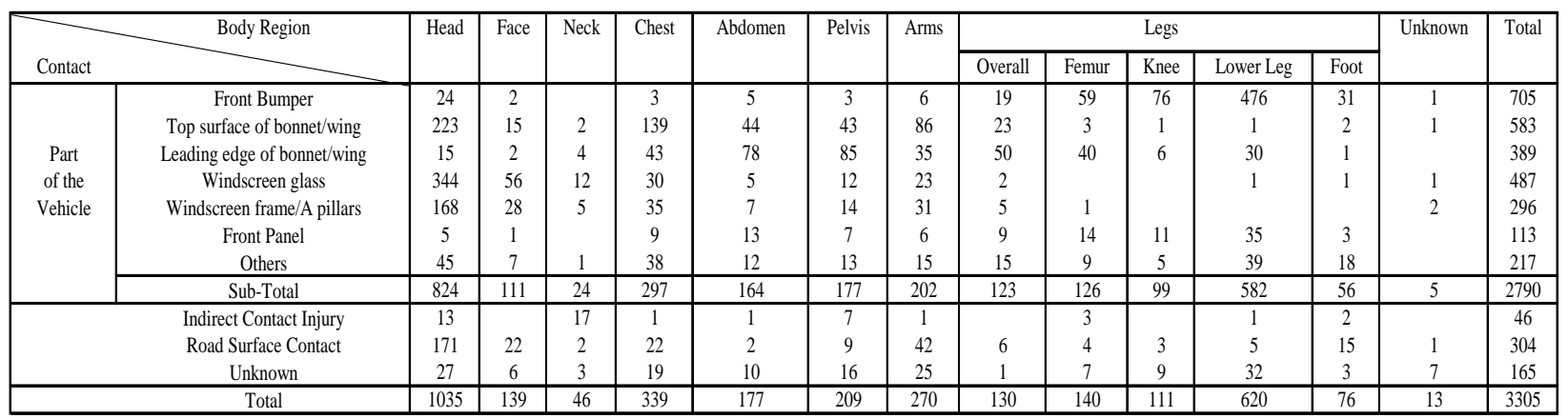

\section{$\underline{\text { Head Kinematics }}$}

Fatal and severe head injuries are most frequently caused by head impact with the bonnet and/or windshield frame. The bonnet rear and scuttle, the bonnet-fender, the rigid accessory to the engine compartment and the windshield frame areas are stiff in current car designs. The head impact conditions consist of head impact speed relative to the car, head impact angle relative to the car, and head impact location. The head impact speed and angle are calculated by the following formulas, where $\mathrm{V}_{\mathrm{y}}$ is the relative head horizontal velocity against the vehicle and $\mathrm{V}_{\mathrm{z}}$ is the relative head vertical velocity against the vehicle (Chen et al. 2009). The head impact location is measured with wrap around distance (WAD).

$$
\begin{aligned}
& V=\left(V_{y}^{2}+V_{z}^{2}\right)^{1 / 2} \\
& \alpha=\arctan \left(V_{z} / V_{y}\right)
\end{aligned}
$$




\section{$\underline{\text { Head Injury Mechanism }}$}

Common pedestrian head injury patterns are skull fracture and brain injuries (Yang 2005). Clinically, brain injuries can be classified in two broad categories: focal injuries and diffuse injuries. The focal injuries consist of epidural hematomas (EDH), subdural hematomas (SDH), intracerebral hematomas (ICH), and contusions (coup and countercoup). The diffuse injuries consist of brain swelling, concussion, and diffuse axonal injury (DAI).

When the head of a pedestrian is struck by a car front, three injury mechanisms can take place because of: the concentrated compressive force, the viscous loading within the skull and the inertial loading to the head/brain (Table 2.3). A similar description on head injury mechanisms was shown in Figure 2.10 with different colors (Gennarelli et al. 1989; Arregui 2006).

Table 2.3 Summary of the typical head/brain injuries and related injury mechanisms (Yang 2005)

\begin{tabular}{|c|c|}
\hline Head injuries & Mechanisms \\
\hline Skull fractures & Contact forces \\
\hline \multicolumn{2}{|c|}{ Intracranial vascular injuries due to ruptured arteries and/or bridging veins } \\
\hline Coup contusion & Occur at the impact point due to contact forces \\
\hline Contrecoup contusion & $\begin{array}{l}\text { Occur at remote site from the impact due to contact force and } \\
\text { deceleration }\end{array}$ \\
\hline Extradural hematoma & Contact force at the impact location \\
\hline Subdural hematoma & Head translational and rotational acceleration \\
\hline \multicolumn{2}{|c|}{ Brain neurological injuries due to tearing of neuronal axons in the brain tissues } \\
\hline $\begin{array}{l}\text { Concussion } \\
\text { Diffuse axonal injury }\end{array}$ & $\begin{array}{l}\text { Rotational motion, relative motion between skull and brain } \\
\text { Rotational motion }\end{array}$ \\
\hline
\end{tabular}



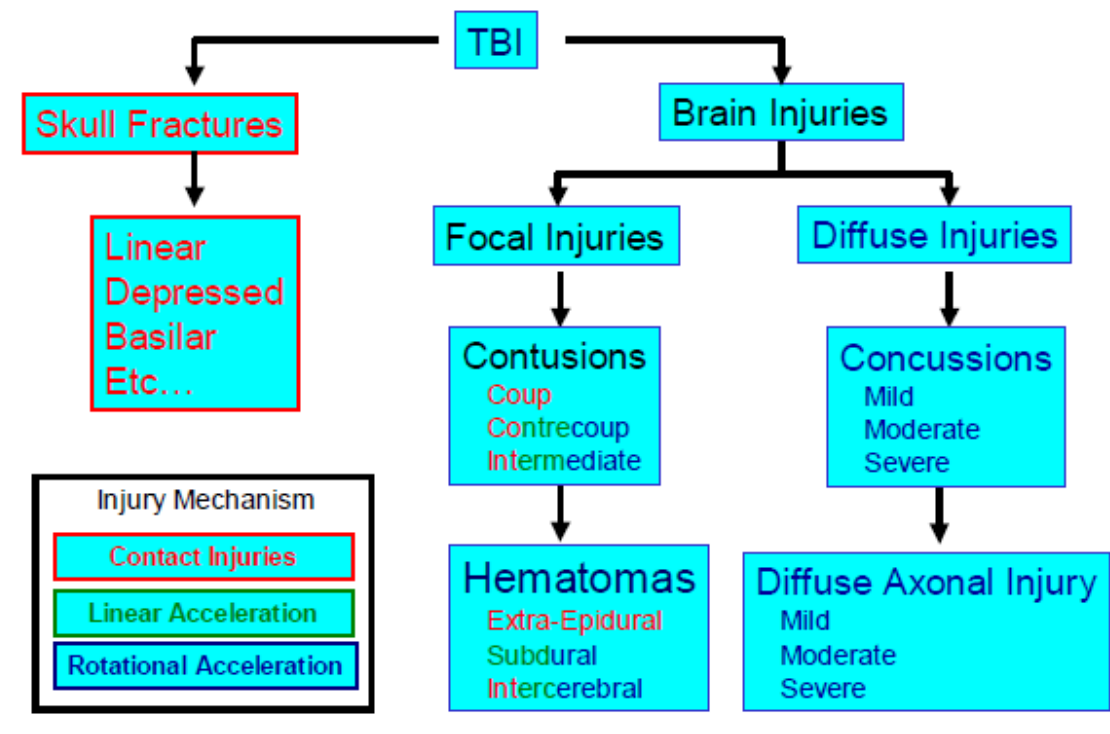

Figure 2.10 Head injuries versus injury mechanisms (Gennarelli et al. 1989; Arregui 2006)

The skull fracture depends mainly on the impact location on the skull and the contact area, such as the bonnet top and windshield frame, and when the impact force exceeds the tolerance level cranial bone fracture could occur. The skull fracture can occur with or without brain injury.

Both the diffuse injuries and the focal injuries may arise from either inertial loading of the head or local deformations in the skull. When the head is subjected to inertial loading in an acceleration field, there will be a relative movement between the skull and the brain. The brain injuries, such as DAI, can result from a high shearing strain and strain rate. The pedestrian head contact with the car front is subjected to both a direct impact force and an acceleration or deceleration, so that the pedestrian can have a complex skull/brain injury from the combined impact responses.

\section{$\underline{\text { Head Injury Criteria }}$}

Traumatic injuries occur when the mechanical tolerance limits of body structures are exceeded. Injuries are generally believed to result from excess strain induced by direct or indirect (i.e., inertial) loading and the most important advance in biomechanics has been the development 
of injury criteria relating the probability of trauma to mechanical parameters which can be measured using instrumented crash test dummies or cadavers, or evaluated in a numerical model. Without injury criteria, the severity of trauma in a staged test or an accident reconstruction cannot be evaluated.

The head injury covers many potential traumatic events and no single mechanical parameter can predict all of them. However, so far, there is only one head injury criteria which is widely used, and this is the Head Injury Criterion (HIC), which is based solely on the time history of linear acceleration of the center of gravity of the head. The HIC was developed from the Wayne State Concussive Tolerance Curve (Simms et al. 2009), which showed that the linear acceleration required for skull fracture is inversely related to impact duration. Since skull fracture is correlated with moderate concussion, the WSTC was proposed as a predictor for head injury. HIC includes the effect of acceleration time history a(t) and duration of the acceleration, and it is defined as:

$$
H I C=\left\{\left(t_{2}-t_{1}\right)\left[\frac{1}{t_{2}-t_{1}} \int_{t_{1}}^{t_{2}} a(t) d t\right]^{2.5}\right\}_{\max }
$$

Where $t_{1}$ and $t_{2}$ are the initial and final times (in seconds) of the interval during which HIC attains a maximum value. When the acceleration is expressed in g's, a HIC value of 1000 has an approximately 50\% probability of skull fracture, and is specified as the level for onset of severe head injury. The time duration of HIC is limited to a specific value, usually 15 or 36 ms.

The HIC is very sensitive to high frequency noise in the head acceleration measurement, and the 3ms criterion, also based on the WSTC, was therefore developed. The $3 \mathrm{~ms}$ threshold is 
the maximum acceleration sustained for at least 3 milliseconds, and a threshold of $80 \mathrm{~g}$ has been proposed for skull fracture (Got et al. 1978).

Cadaver tests data showed good correlation between head injury severity and HIC (McIntosh et al. 1993; MacLaughlin et al. 1993), and pedestrian accident reconstruction for adult and child also showed strong correlation between HIC score and death and injury (Kessler and Monk 1991; Yao et al. 2007). However, there have been no direct demonstrations of functional brain damage to accompany the skull fracture in the Wayne State tests (Simms and Wood, 2009), and the HIC has been frequently criticized.

A major flaw in the HIC is that it only accounts for linear acceleration. This is appropriate for skull fracture predictions, but ignoring the effect of rotational kinematics makes the HIC theoretically unsuitable for shear strain induced brain injury prediction. In 1943, Holbourn proposed one of the most comprehensive theories for a brain injury mechanism, using the fundamental laws of motion to describe the interaction between the skull and brain during a head impact (Holbourn 1943). Combined with the near incompressible nature of brain tissue, Holbourn's theory makes rotational kinematics seem well suited for studying closed-head brain injuries. After that, numerous studies have focused on the development of rotational-based brain injury tolerance.

More recently, the FE models of the human head provided the most suitable tools for studying the complex, tissue-level mechanical response of the brain during a head impact (Takhounts et al. 2013, 2008). Two strain-based brain injury metrics have been used in previous computational studies to categorize brain injury: maximum principle strain (MPS) and Cumulative Strain Damage Measure (CSDM) (Takhounts et al. 2013). Gabler et al. (2016) assessed fifteen kinematic-based brain injury metrics by comparing their correlation to tissue- 
level strain responses obtained from 660 head impacts simulated in two separate human head FE models (SIMon and GHBMC head model) (Takhounts et al. 2003, 2008), and two angular velocity-based metrics, the Brain Injury Criterion (BrIC) and Rotational Velocity Change Index (RVCI), were found to have the highest overall correlation with closed head injuries. The BrIC, is the updated criterion to the original BRIC formulation after finding that angular velocity alone was sufficient to predict FE model strains (Takhounts et al. 2013), and is formulated using the maximum magnitudes of the three orthogonal head angular velocity components:

$$
\mathrm{BrIC}=\sqrt{\left(\frac{\omega_{x}}{\omega_{x \mathrm{cr}}}\right)^{2}+\left(\frac{\omega_{y}}{\omega_{y \mathrm{cr}}}\right)^{2}+\left(\frac{\omega_{z}}{\omega_{z \mathrm{cr}}}\right)^{2}}
$$

Where $\omega_{\text {icr }}$ are directionally dependent critical values that were determined using $\mathrm{E}$ modeling (Takhounts et al. 2013). The critical values for three-dimensional angular velocities were chosen such that a BrIC value of 1.0 corresponded to a risk of $50 \%$ for AIS4+ brain injury. Based on critical values derived from CSDM and maximum principal strain (MPS), the suggested angular velocity thresholds $\omega_{i, \text { crit }}$ were $\omega_{x, \text { crit }}=66.25 \mathrm{rad} / \mathrm{s}, \omega_{y, \text { crit }}=56.45 \mathrm{rad} / \mathrm{s}$ and $\omega_{z, c r i t}=42.87 \mathrm{rad} / \mathrm{s}$.

\subsection{Method and Tools for Pedestrian Head Injury Research}

To understand the pedestrian injury mechanism and reduce the pedestrian injury risk, numerous efforts were made during the last decades, including physical tests, mathematical modeling, regulation development, and improving vehicle design. This section comprehensively reviews the methods and tools that have been used and presents their advantages and disadvantages, discusses pedestrian regulation development and its limitation, and countermeasures made in the industry. 


\subsubsection{Physical Test}

Biomechanical tests on component and full-scale level are important to improve the understanding of pedestrian kinematics and injuries as well as for the evaluation of test tools. Traditionally, many full-scale pedestrian cadaver tests (Paas et al. 2016; Forman et al. 2015; Kerrigan et al. 2005, 2007; Subit et al. 2008) and head (Yoganandan et al. 2004) or lower extremity (Kerrigan et al. 2008) component test were conducted. Recently, due to ethical concerns and cost restrictions for biomechanical tests, crash test dummies, impactors and human body models were developed, validated, and widely used, though their biofidelity evaluation relies heavily on the PMHS test data. In the future, once fully validated, these crash dummies and mathematical models might be expected to replace biomechanical tests.

\section{Full-scale test}

Pedestrian full-scale PMHS tests began in the 1970s, Pritz et al. (1975) examined the influence of vehicle design on pedestrian injury. After that, other notable early full-scale pedestrian PMHS tests have been conducted before 2000s by Césari et al. (1980), and Ishikawa et al. (1993), etc. Recent PMHS tests provided more detailed data on pedestrian anthropometry, initial position and posture, and time history of cadaver response. Subit et al. (2008) studied the kinematics and injuries of four PMHSs, where two short and two tall subjects were impacted by a small city car (Figure 2.11) and a mid-sized sedan. The study focused on the pelvis and upper body kinematics, which were found to depend on subject size and vehicle front geometry. Forman et al. (2015) conducted three PMHS tests against a generic buck to develop normalized trajectory corridors for the biofidelity evaluation of dummy or HBM. Detailed three dimensional data were provided in these tests. In the other study, Pass et al. (2016) also provided detailed 
three dimensional pedestrian initial positions and response, and unlike most of the previous tests the arms of the pedestrian were not bounded.
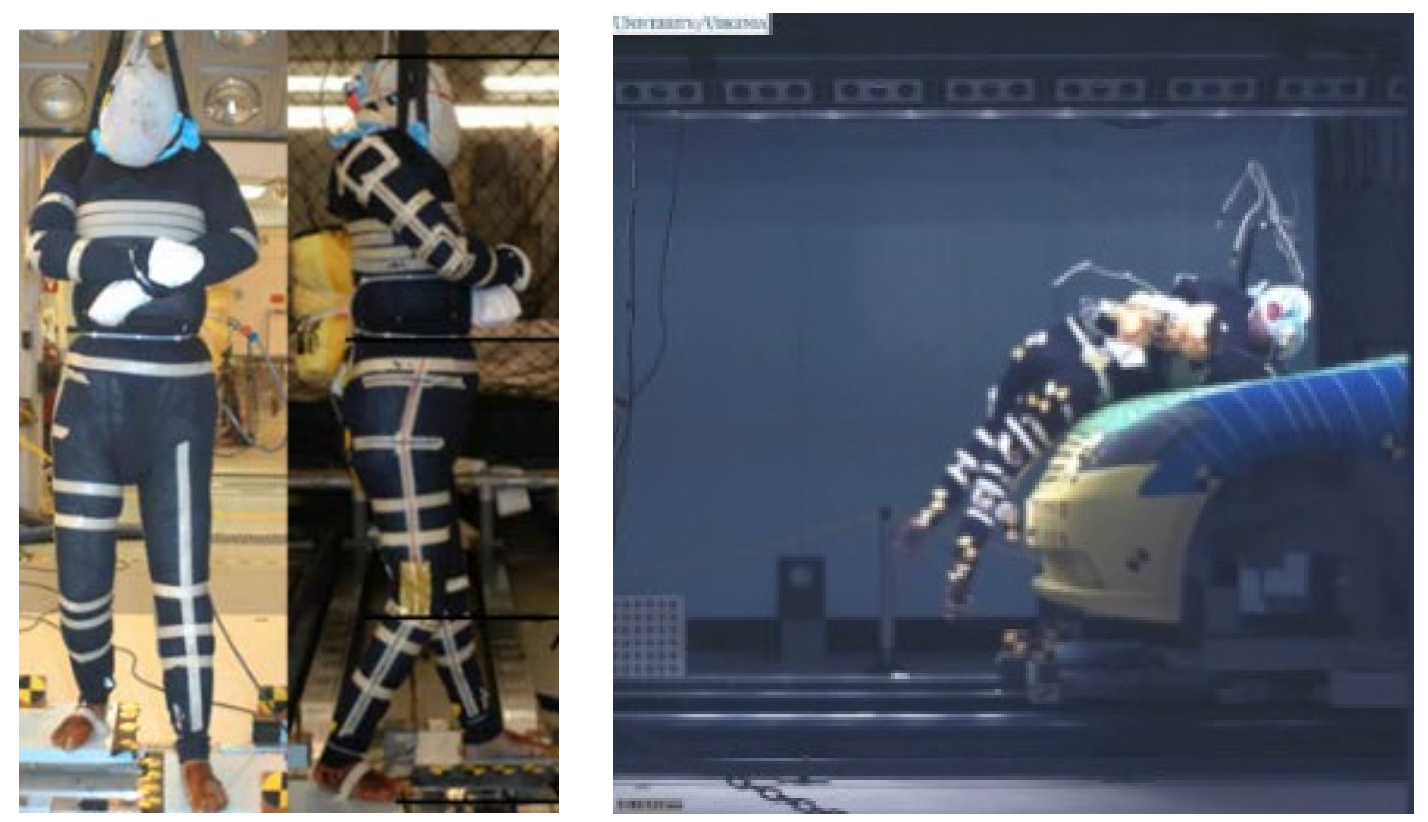

Figure 2.11 Full-scale vehicle-pedestrian cadaver test (Subit et al. 2008)

Full-scale PMHS tests can be used to reconstruct pedestrian kinematics and, to some levels, injuries of pedestrian. One of the most notable contributions of these full-scale PMHS tests, especially those most recent ones, is that they provided detailed and biofidelic data for evaluation of pedestrian crash dummy and mathematical human models. Nevertheless, there are still many issues and limitations of PMHS test data, including the high cost and ethical issues. Also, they provided relatively small sample sizes, and all of these tests focused on only specific impact scenarios such as pure lateral orientation, and the arm of most of cadavers in the tests were bounded while this is not the same with the real accidents. Finally, human subjects cannot be repeatedly used for impact, and huge variability among subjects, including anthropometry and bone quality makes it not suitable for sensitivity study. These limitations make it difficult to use to evaluate the modifications in vehicular frontal structure to reduce pedestrian injury risk. 
Another way to assess the vehicle aggressiveness to pedestrian was the development of new pedestrian dummies. One of the physical test devices in pedestrian safety is the Hybrid III pedestrian dummy, which is updated from the occupant dummy by making modification in the pelvis, lower torso, and knee region. Autoliv and Chalmers University of Technology developed the Chalmers-Autoliv adult pedestrian dummy and The 6 years old (6YO) pedestrian dummy. The head and neck of the adult dummy are adapted from Eurosid-1, the thorax and lumbar spine from US-SID, the pelvis from Hybrid II and lower extremity parts are taken from Hybrid III. A standing position pelvis was added and the knee joints were redesigned and assigned the bending characteristics corresponding to the EEVC WG17 legform impactor to achieve humanlike lateral bending. The dummy was validated against mathematical human body pedestrian model and showed comparable results (Bjorklund and Zheng, 2001; Yao, 2010). The child dummy was based on a 6YO Hybrid III dummy which was equipped with a standard neck originally designed for frontal impact tests (Hayamizu et al., 2002). The neck was modified to provide higher biofidelity to the neck in lateral bending.

Honda developed and manufactured the POLAR II pedestrian dummy (Figure 2.), which is modified from the THOR occupant dummy in its body structure to reproduce human body kinematics for $30 \mathrm{~km} / \mathrm{h}$ to $50 \mathrm{~km} / \mathrm{h}$ impact velocities. POLAR II has instruments throughout the body to measure the risk of injury. In the injury prone lower leg area, it has a detailed knee design that incorporates femoral condyles, meniscus, tendons and ligaments (Akiyama et al., 1999). Kerrigan et al. (2005) and (2007) investigated the kinematics of PMHSs and the Polar-II dummy in impacts against two different mid-size sedan cars and an SUV (Figure 2.12) in an attempt to establish kinematic corridors by scaling time, as well as the trajectories for each body segment. One of their findings was that the PMHSs generally showed longer Wrap Around 
Distances (WADs) than the dummy, and which they attributed partly to the PMHSs' tendency to slide more over the bonnet than the dummy, and partly to the lack of muscle tension in the PMHSs. The main reasons for a greater lateral bending stiffness in the dummy compared with the PMHSs appears to have been dummy durability and previous dummy component design (Akiyama et al. 2001) although it can also be argued that due to muscle tension, living humans probably have a greater lateral bending stiffness than PMHSs. While the PMHSs' heads lagged behind the upper torso during the upper body rotation over the bonnet towards the vehicle, the head lag was not as pronounced in the dummy, which Kerrigan et al. (2005) attributed to a greater neck bending stiffness in the dummy compared with the PMHSs.
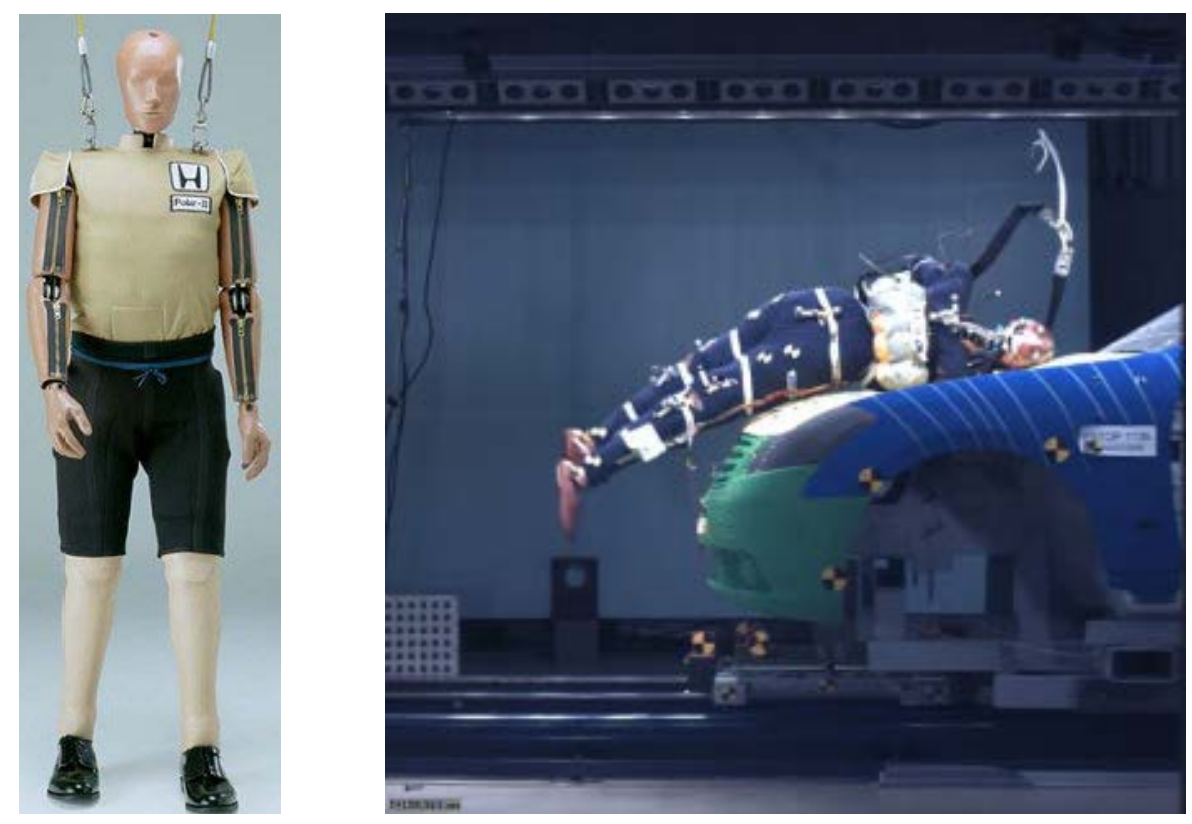

Figure 2.12 Polar II Pedestrian Crash Test Dummy and full-scale vehicle-pedestrian dummy test (Kerrigan et al. 2005)

The pedestrian crash dummy provided a repeatable tool for full-scale impact to evaluate vehicle passive safety. However, their biofidelity needs to be more comprehensively evaluated and improved before being more widely used. Also, the physical dummies can only reflect 
several very specific anthropometries of pedestrian, which is a big issue because pedestrian response highly depends on their anthropometry (Paas et al 2015; Kerrigan et al 2007; Subit et al. 2008).

\section{$\underline{\text { Pedestrian Sub-system Test Procedure }}$}

To reduce the number of pedestrian fatalities, the pedestrian sub-system test procedures were proposed and used to assess the vehicle aggressiveness. Unlike the occupant safety evaluation, the pedestrian full-scale dummy based test methods are not well suited for regulatory purpose, because they would require an unrealistically large number of tests to cover all possible contact areas due to the range of pedestrian stature and vehicle width. The sub-system test methods consist of a set of individual tests, each representing one of the contacts that occur in a vehicle-pedestrian accident. Collectively, the family of sub-system tests represents all of the significant contacts within an accident that are likely to result in serious or fatal injuries to the pedestrian.

The current sub-system test procedure has been developed for more than 20 years. The European Enhanced Vehicle-safety Committee (EEVC) pedestrian test methods were first developed by Working Group 10 (EEVC WG10) and then further refined by Working Group 17 (EEVC WG17). The pedestrian safety tests proposed by EEVC include Lower legform to bumper test, Upper legform to hood leading edge test, Child headform impact to hood top, and Adult headform to hood top (Figure 2.13). Essentially the test concerning the level of protection for the head consists of firing a child headform into the front part of the bonnet top and an adult head into the rear part of the bonnet top. The child headform has a mass of $2.5 \mathrm{~kg}$ and is fired at a velocity of $11.1 \mathrm{~m} / \mathrm{s}$ and at an angle of 50 degrees (relative to the horizontal) into the child zone (1000 to $1500 \mathrm{~mm}$ WAD (wrap around distance)). The adult headform has a mass of $4.8 \mathrm{~kg}$ and 
is fired at a velocity of $11.1 \mathrm{~m} / \mathrm{s}$ and at an angle of 65 degrees (relative to the horizontal) into the adult zone (1500 to $2100 \mathrm{~mm}$ WAD). For both the child and the adult, the head protection criteria are for the Head Injury Criterion (HIC) to be 1000.

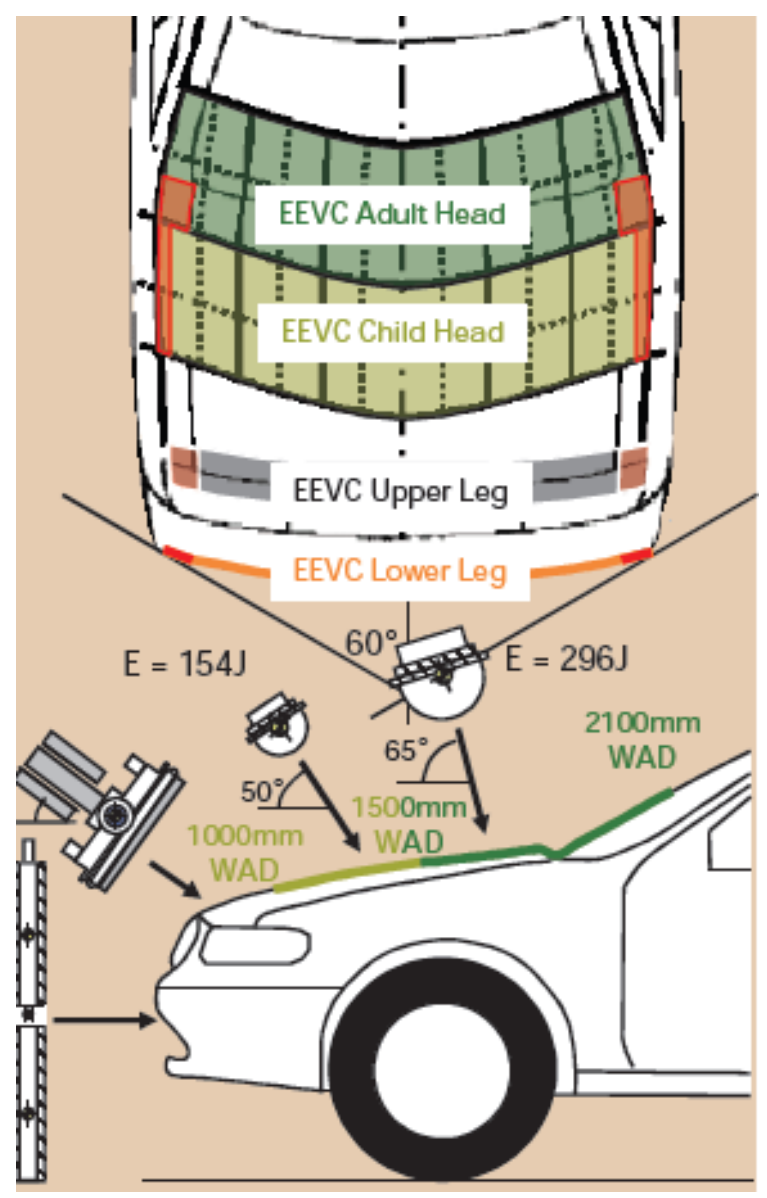

Figure 2.13 EEVC test setups and impact zones

ISO WG2 has also produced sub-system head test methods which are an adaptation of the EEVC test methods. These ISO headform specifications were used as the basis of the draft International Harmonised Research Activities - Pedestrian Safety Working Group (IHRA PSWG) head test method and the IHRA specification for the $3.5 \mathrm{~kg}$ child and $4.5 \mathrm{~kg}$ adult headforms is essentially the same as that of ISO. 
There are different regulations for pedestrian safety and some rating systems for consumer rating of vehicles. Some of the regulations are European Union Directive also known as European Legal, Japan Technical Standard (Japan Legal) and Global Technical Regulation (GTR). Apart from these regulations for pedestrian safety, there are some rating tests developed by Euro-NCAP (European New Car Assessment program), US NCAP, Japan NCAP and Australian NCAP. The test procedures of these Legal and NCAP are all based on the testing methods of these three working groups, with some differences on details.

Under the GTR, head impact tests are performed on the bonnet of the vehicle, but not the windscreen or A-pillar. The side borders of the testable area are defined by drawing reference lines, based on the angle of the vehicle surface. The testable area generally includes the bonnet top, but may also partially include the top of the front wheel guards. The testable area is divided into two sections, based on a specified 'wrap-around distance'. The wrap-around distance is measured from the ground at the front of the vehicle, with a flexible measuring tape along the vehicle surface. The area that lies between wrap-around distances of 1000 and $1700 \mathrm{~mm}$ is tested with a $3.5 \mathrm{~kg}$ 'child' headform at an angle of $50^{\circ}$ to the horizontal. The area between 1700 and $2100 \mathrm{~mm}$ is tested with a $4.5 \mathrm{~kg}$ 'adult' headform at an angle of $65^{\circ}$ to the horizontal. These wrap-around distances define the areas that the head of a pedestrian is likely to strike in a typical collision.

The most potentially harmful locations are selected within the testable area, and are tested with the appropriate headform. A minimum of nine headform tests are performed in each of the child and adult test areas. Any test that results in a HIC of less than 1000 is considered to pass the GTR requirements. Before testing begins, the manufacturer may nominate up to one third of the testable area to be defined as a so-called 'relaxation zone'. The relaxation zone may include 
any part of the testable area and does not need to be contiguous. The relaxation zone cannot consist of more than one half of the child testing area. For tests in the relaxation zone, the HIC may be up to 1700 and still pass the GTR requirements. In the remainder of the testable area, the HIC may not exceed 1000. The headform impact speed specified by the GTR is $35 \mathrm{~km} / \mathrm{h}$, which differs from the speed of $40 \mathrm{~km} / \mathrm{h}$ specified in the EuroNCAP protocol. In all other respects, the test conditions specified in the current EuroNCAP procedure are similar to the GTR. In the previous version of the EuroNCAP procedure, which was used up until the end of 2009, the child headform was $2.5 \mathrm{~kg}$ and the adult headform was $4.8 \mathrm{~kg}$, which differ from the GTR headform masses. In this previous version, the dividing line between child and adult was $1500 \mathrm{~mm}$, not the $1700 \mathrm{~mm}$ used in the GTR and the current EuroNCAP procedure.

The EEVC WG17 developed headform impactors for pedestrian safety tests of passenger cars for the European Commission. Earlier two different headform impactors for adult head and child head were developed with different sizes $(165 \mathrm{~mm}$ in diameter for adult and $135 \mathrm{~mm}$ in diameter for child). New headform impactors were developed with both headform of the same size with the adult head-form impactor of $4.5 \mathrm{~kg}$ and child head-form impactor of $3.5 \mathrm{~kg}$. Construction of the headform consists of an inner hollow sphere made of aluminum, an end plate made of aluminum and a skin constructed by PVC (polyvinyl chloride) and has mount for accelerometer to track head accelerations in three mutually perpendicular directions (Figure 2.14). These headform impactors are tested for biofedility (skin stiffness) using a drop test with a test corridor of 225-275 G for adult headform impactor and test corridor of 240-300 G for child headform. Figure 2.14 shows the construction details for the headform impactor. These headform impactors are good for parametric study in initial vehicle front-end design and to get some idea of head acceleration and HIC number in early phases. But these impactors being free motion 
headform can't predict the actual kinematics of neck-head response during impact and thus are limited in actual level of injury prediction.
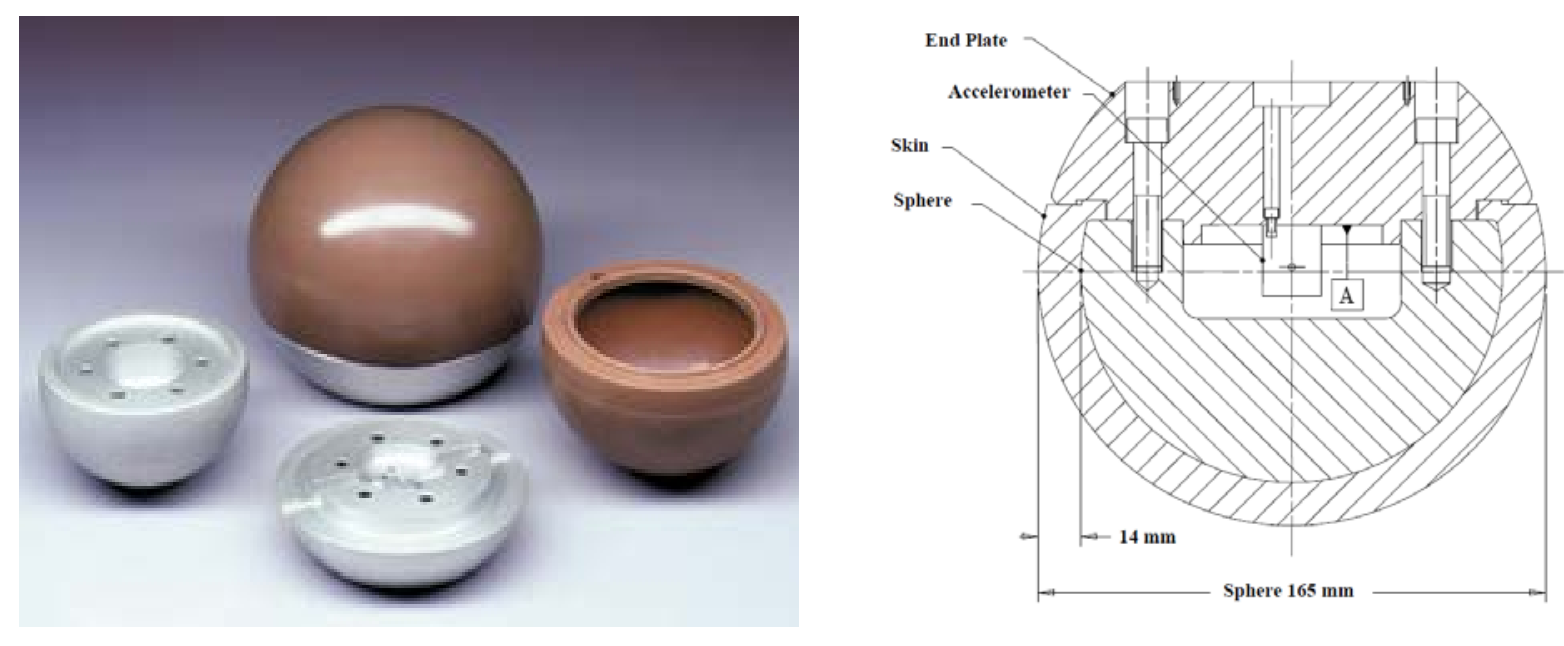

Figure 2.14 Adult headform impactor

Compared to the full-scale dummy test, the advantage of the sub-system test is that it can test all the potential impact locations, with better repeatability and much lower cost. However, there are two fundamental problems for the component test.

First, for the input of the test procedure, head impact conditions, including the impact location, impact speed, angle, and effective mass, cannot be obtained without the full-scale impact system. Therefore, there is the need to reproduce them in some other way. With this issue, the three working groups, EEVC (Janssen and Nieboer 1990; Glaeser 1991), ISO (Lawrence and Harris 1988; Ishikawa et al 1993), and IHRA (Mizuno 2003; Mizuno 2005) used PMHS tests and multi-body simulations to generate the head impact conditions as the input of pedestrian subsystem test procedure. Most of these studies were targeted at the pedestrian accidents with a vehicle travel speed of $40 \mathrm{~km} / \mathrm{h}$. However, those studies proposed inconsistent head impact conditions, and very wide ranges were observed with the head impact speed ranging from 0.4-1.5 times of vehicle travel speed, head impact angle from $48^{\circ}$ to $108^{\circ}$, and head effective mass from 
4.5 to $6.5 \mathrm{~kg}$, which challenged the selection of appropriate values to represent the realistic pedestrian head impact (Hardy et al. 2007). Due to these limitations, the current head impact conditions were deemed provisional (Hardy et al. 2007).

Second but more important, because of the complexity of pedestrian kinematics during vehicle impact, subsystem test procedures using local impactors are insufficient to evaluate the comprehensive level of protection potentially afforded by vehicle countermeasures. For example, the energy associated with a head hitting a car in the case of a head impactor is smaller than the mass of a full pedestrian (which includes torso, etc.). The pedestrian can pre-deform the hood with the body losing part of the efficiency. The change on the vehicle design to obtain better performance in the component test could actually also change the head impact conditions in the realistic full-scale impact, but this is not fully considered in the current test procedure. Other differences include the lack of head-neck interaction, head rotational kinematics, head impact geometry, headform biofidelity, and so on. These factors that are missed in the component test make the capability of the simplified two-dimensional component test to correctly represent the realistic full-scale impact questionable. Furthermore, a big potential problem might exist that better performing vehicles in current sub-system procedure will not necessarily obtain lower head injury risk in realistic full-scale pedestrian impact.

\subsubsection{Mathematical Models}

Both dummies and impactors are themselves highly simplified models of actual humans, which may lead to injury assessments that differ from those obtained in PMHS tests (Kerrigan et al. 2005, Kerrigan et al. 2008). Additionally, safety systems should protect a large range of the population, which can more easily be represented by HBMs, while anthropometric changes to a 
dummy are more difficult to introduce. In contrast, because HBMs are relatively easy to adjust and modify, they can be used to study many different impact conditions without causing physical damage to real vehicles, crash test dummies or PMHSs, which makes HBMs suitable tools for variation studies and for early stages of product development by car manufacturers. For all these reasons, HBMs are invaluable tools for pedestrian safety system development and evaluation.

In the last decades, more and more mathematical models were developed, validated, and widely used to investigate pedestrian injury mechanism and evaluate vehicle passive safety performance. These models are mainly based on two theories: multi-body or finite element method.

\section{Multi-Body Modeling}

Multi-Body (MB) models are usually composed of rigid body ellipsoids and planes with a point mass in their centers of gravity and with inertial properties assigned to them. These bodies are connected by joints with a lumped parameter joint stiffness, simulating the interaction between bones, muscles, and ligaments. Contact and penetration characteristics are approximated by idealized functions. This approach allows for low computation time. The level of detail in MB models is lower than in FE models, and their tissue-level injury prediction capabilities are limited. Compared to FEM models, multi-body models are much more efficient in predicting the gross motion of pedestrian body segments. It is therefore considered a valuable tool to evaluate the influence of vehicle front shape and stiffness properties on pedestrian injury risks.

Yang et al. (2000) developed a series of complete pedestrian models with a human-like knee joint and breakable leg model. The results from a sensitive study indicated that these pedestrian models are capable of predicting the human-like kinematics and impact responses of various body segments. Other MB pedestrian models developed include TNO pedestrian models 
(Automotive, T.N.O. 2001), JARI pedestrian models, NHTSA pedestrian models, and RARU pedestrian models (Neale et al. 2005) (Figure 2.15).

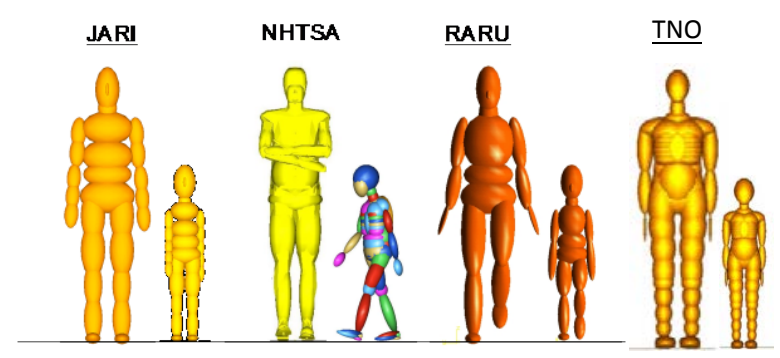

Figure 2.15 The multi-body pedestrian human models

These MB models provided useful tools to investigate the pedestrian global kinematics, and thus were widely used to study pedestrian head impact conditions by the three working groups mentioned above. In those three working groups, the most recent study on pedestrian head impact conditions is from the IHRA. In this study, the front shapes of passenger cars were investigated and categorized into three groups, Sedan, SUV (Sport Utility Vehicle) and 1-Box (One Box Vehicle), so that the effect of vehicle front shapes on the pedestrian impact were studied with computer simulations focusing on the head impact velocity, head impact angle, WAD (Wrap Around Distance) and head effective mass. Three MB human models were used, including JARI model, TNO model, and the RARU model. The results for the output parameters relating to head impacts with the bonnet and the windscreen are summarized in Table 2.4, for three categories of passenger cars. The average head impact speeds were found to be lower than vehicle travel speed in these simulations. 
Table 2.4 Summary of Parameter Study for Adult pedestrian (Mizuno 2003)

For Adult

\begin{tabular}{|c|c|c|c|c|c|c|}
\hline \multirow[t]{3}{*}{$\begin{array}{l}\text { Shape } \\
\text { Corridor }\end{array}$} & \multicolumn{6}{|c|}{$\begin{array}{c}\text { Car impact speed } \\
30 \mathrm{~km} / \mathrm{h}\end{array}$} \\
\hline & \multicolumn{3}{|c|}{$\begin{array}{l}\text { Impact Velocity } \\
(\mathrm{km} / \mathrm{h})\end{array}$} & \multicolumn{3}{|c|}{$\begin{array}{l}\text { Impact Angle } \\
\text { (deg.) }\end{array}$} \\
\hline & Bonnet & Windsheld & BLE/Grille & Bonnet & Windsheld & BLE/Grille \\
\hline $\begin{array}{l}\text { Sedan + } \\
\text { SUV } \\
\text { One box }\end{array}$ & $\begin{array}{rrr}23.7 & +/- & 6.0 \\
26.4 & +/- & 3.6 \\
& \text { nc } & \end{array}$ & $\begin{array}{rrr}27.3 & +/- & 5.4 \\
& \text { nc } & \\
20.4 & +/- & 3.6\end{array}$ & $\begin{array}{l}\text { nc } \\
\text { nc } \\
\text { nc }\end{array}$ & $\begin{array}{ccc}78.3 & +/- & 5.6 \\
73.8 & +/- & 21.5 \\
& n c & \end{array}$ & $\begin{array}{ccc}48.8 & +/- & 9.9 \\
& n c & \\
55.1 & +/- & 10.4\end{array}$ & $\begin{array}{l}\text { nc } \\
\text { nc } \\
\text { nc }\end{array}$ \\
\hline
\end{tabular}

\begin{tabular}{|c|c|c|c|c|c|c|}
\hline \multirow[t]{3}{*}{$\begin{array}{l}\text { Shaep } \\
\text { Corridor }\end{array}$} & \multicolumn{6}{|c|}{$\begin{array}{c}\text { Car impact speed } \\
40 \mathrm{~km} / \mathrm{h}\end{array}$} \\
\hline & \multicolumn{3}{|c|}{$\begin{array}{l}\text { Impact Velocity } \\
(\mathrm{km} / \mathrm{h})\end{array}$} & \multicolumn{3}{|c|}{$\begin{array}{l}\text { Impact Angle } \\
\text { (deg.) }\end{array}$} \\
\hline & Bonnet & Windsheld & BLE/Grille & Bonnet & Windsheld & BLE/Grille \\
\hline $\begin{array}{l}\text { Sedan + } \\
\text { SUV } \\
\text { One box }\end{array}$ & $\begin{array}{rrr}30.4 & +/- & 7.2 \\
30.8 & +/- & 8.8 \\
\text { nc } & \end{array}$ & $\begin{array}{rrr}35.2 & +/- & 6.8 \\
& \text { nc } & \\
29.6 & +/- & 3.2 \\
\end{array}$ & $\begin{array}{l}\mathrm{nc} \\
\mathrm{nc} \\
\mathrm{nc}\end{array}$ & $\begin{array}{ccc}66.0 & +/- & 14.0 \\
76.7 & +/- & 22.2 \\
& \text { nc } & \end{array}$ & $\begin{array}{ccc}38.4 & +/- & 10.9 \\
& \text { nc } & \\
47.3+/- & 9.6\end{array}$ & $\begin{array}{l}\text { nc } \\
\text { nc } \\
\text { nc }\end{array}$ \\
\hline
\end{tabular}

\begin{tabular}{|c|c|c|c|c|c|c|}
\hline \multirow[t]{3}{*}{$\begin{array}{l}\text { Shaep } \\
\text { Corridor }\end{array}$} & \multicolumn{6}{|c|}{$\begin{array}{c}\text { Car impact speed } \\
50 \mathrm{~km} / \mathrm{h}\end{array}$} \\
\hline & \multicolumn{3}{|c|}{$\begin{array}{l}\text { Impact Velocity } \\
(\mathrm{km} / \mathrm{h})\end{array}$} & \multicolumn{3}{|c|}{$\begin{array}{l}\text { Impact Angle } \\
\text { (deg.) }\end{array}$} \\
\hline & Bonnet & Windsheld & BLE/Grille & Bonnet & Windsheld & BLE/Grille \\
\hline $\begin{array}{l}\text { Sedan + } \\
\text { SUV } \\
\text { One box }\end{array}$ & $\begin{array}{ccc}37.5 & +/- & 9.5 \\
39.5 & +/- & 11.0 \\
& n c & \end{array}$ & $\begin{array}{rrr}46.5 & +/- & 11.0 \\
& \text { nc } & \\
43.0+/- & 6.0\end{array}$ & $\begin{array}{l}\mathrm{nc} \\
\mathrm{nc} \\
\mathrm{nc}\end{array}$ & $\begin{array}{ccc}56.8 & +/- & 11.5 \\
73.5 & +/- & 25.2 \\
& n c & \end{array}$ & $\begin{array}{ccc}33.5 & +/- & 11.3 \\
& \text { nc } & \\
38.4+/- & 12.3\end{array}$ & $\begin{array}{l}\text { nc } \\
\text { nc } \\
\text { nc }\end{array}$ \\
\hline
\end{tabular}

*nc: No Contact

** Linear interpretation to be used to determine impact conditions for in-between speeds if required.

In the other study, Chen et al. (2009) conducted similar simulations using only JARI pedestrian model, while the vehicle front shapes were derived from an investigation of Chinese pedestrian accidents. The head impact speeds were found to be very close to vehicle traveling speeds in this study (Table 2.5 - 2.6). 
Table 2.5 Pedestrian Head Impact Conditions (Adult, 40km/h) (Chen et al. 2009)

\begin{tabular}{|c|c|c|c|c|c|c|c|c|c|}
\hline & \multicolumn{3}{|c|}{ Sedan } & \multicolumn{3}{|c|}{ SUV } & \multicolumn{3}{|c|}{ 1-Box } \\
\hline & Lower & Middle & Upper & Lower & Middle & Upper & Lower & Middle & Upper \\
\hline $\begin{array}{l}\text { Head impact } \\
\text { location }\end{array}$ & $\begin{array}{l}\text { Wind } \\
\text { screen }\end{array}$ & $\begin{array}{l}\text { Wind } \\
\text { screen }\end{array}$ & $\begin{array}{l}\text { Wind } \\
\text { screen }\end{array}$ & bonnet & bonnet & bonnet & $\begin{array}{l}\text { Wind } \\
\text { screen }\end{array}$ & $\begin{array}{l}\text { Wind } \\
\text { screen }\end{array}$ & $\begin{array}{l}\text { Wind } \\
\text { screen }\end{array}$ \\
\hline $\begin{array}{l}\text { Head impact } \\
\text { time(ms) }\end{array}$ & 149.7 & $\begin{array}{l}129.1 \\
\mathbf{1 3 2 . 0}\end{array}$ & 108.4 & 109.4 & $\begin{array}{l}97.3 \\
\mathbf{9 8 . 9}\end{array}$ & 79.4 & 79.0 & $\begin{array}{l}62.8 \\
63.8\end{array}$ & 50.7 \\
\hline $\begin{array}{l}\text { Head impact } \\
\text { velocity }(\mathrm{km} / \mathrm{h})\end{array}$ & 43.3 & $\begin{array}{l}37.7 \\
35.5\end{array}$ & 40.0 & 41.1 & $\begin{array}{l}39.5 \\
38.4\end{array}$ & 41.5 & 36.1 & $\begin{array}{l}35.5 \\
\mathbf{3 4 . 3}\end{array}$ & 24.8 \\
\hline $\begin{array}{l}\text { Head impact } \\
\text { angle }\left(^{\circ}\right)\end{array}$ & 57.1 & $\begin{array}{l}44.8 \\
50.0\end{array}$ & 40.7 & 69.1 & $\begin{array}{l}84.1 \\
\mathbf{8 5 . 0}\end{array}$ & 97.3 & 31.2 & $\begin{array}{l}45.2 \\
45.4\end{array}$ & 66.6 \\
\hline HIC & 925.1 & $\begin{array}{l}844.5 \\
870.7\end{array}$ & 949.2 & 2186.8 & $\begin{array}{l}2073.3 \\
1932.9\end{array}$ & 2542.9 & 1103.0 & $\begin{array}{l}1296.7 \\
\mathbf{1 2 3 9 . 3}\end{array}$ & 1007.2 \\
\hline $\mathrm{WAD}(\mathrm{m})$ & 2.204 & $\begin{array}{l}2.063 \\
2.044\end{array}$ & 1.908 & 1.853 & $\begin{array}{l}1.733 \\
\mathbf{1 . 7 2 9}\end{array}$ & 1.593 & 1.773 & $\begin{array}{l}1.685 \\
\mathbf{1 . 6 8 6}\end{array}$ & 1.591 \\
\hline $\begin{array}{l}\text { Head effective } \\
\text { mass(kg) }\end{array}$ & 4.95 & $\begin{array}{l}4.23 \\
4.07\end{array}$ & 3.93 & 3.32 & $\begin{array}{l}3.30 \\
3.27\end{array}$ & 3.04 & 3.63 & $\begin{array}{l}3.30 \\
3.32\end{array}$ & 2.08 \\
\hline
\end{tabular}

*the bold values are simulation result of braking during impact, ——head contact several location of vehicle

Table 2.6 Pedestrian Head Impact Conditions (for Adult and Child, 30km/h and 50km/h) (Chen et al. 2009)

\begin{tabular}{|c|c|c|c|c|c|c|}
\hline & \multicolumn{3}{|c|}{$30 \mathrm{~km} / \mathrm{h}$} & \multicolumn{3}{|c|}{$50 \mathrm{~km} / \mathrm{h}$} \\
\hline & Sedan(middle) & SUV(middle) & $\begin{array}{c}\text { 1-Box } \\
\text { (middle) }\end{array}$ & $\begin{array}{c}\text { Sedan } \\
\text { (middle) }\end{array}$ & SUV(middle) & $\begin{array}{c}\text { 1-Box } \\
\text { (middle) }\end{array}$ \\
\hline $\begin{array}{l}\text { Head impact } \\
\text { location }\end{array}$ & Windscreen & bonnet & Windscreen & Windscreen & bonnet & Windscreen \\
\hline $\begin{array}{l}\text { Head impact } \\
\text { time(ms) }\end{array}$ & 158.5 & 122.7 & 80.9 & 105.3 & 81.5 & 50.9 \\
\hline $\begin{array}{c}\text { Relative } \\
\text { impact } \\
\text { velocity }(\mathrm{km} / \mathrm{h})\end{array}$ & 31.4 & 30.2 & 25.9 & 48.0 & 48.8 & 45.9 \\
\hline $\begin{array}{c}\text { Relative } \\
\text { impact } \\
\text { angle }\left(^{\circ}\right)\end{array}$ & 60.8 & 86.0 & 50.4 & 38.2 & 79.1 & 37.0 \\
\hline HIC & 788.1 & 1090.4 & 773.3 & 1007.5 & 3852.2 & 2072.7 \\
\hline $\mathrm{WAD}(\mathrm{m})$ & 1.960 & 1.677 & 1.660 & 2.086 & 1.789 & 1.711 \\
\hline $\begin{array}{c}\text { Head effective } \\
\text { mass(kg) }\end{array}$ & 3.88 & 3.08 & 3.35 & 4.66 & 3.25 & 3.30 \\
\hline
\end{tabular}

These simulation studies using MB models provided valuable reference for generating pedestrian head impact conditions for sub-system test procedure. However, there are many 
limitations in those studies. First, multi-body human models used in those studies (JARI model, NHTSA model, RARU model, and TNO model) were found to have serious problems with regard to the biofidelity of the shoulder (Hardy et al. 2007), which was found to greatly influence head response (Chen et al. 2016; Neal et al. 2005). Second, at the time of those studies (EEVC (1990-1991), ISO (1988-1993), IHRA (2003-2005)), multi-body vehicle models with very simplified geometry and stiffness were used, and these vehicle models were based on the oldfashioned front shapes which were more sharp and protruding. Third, those studies didn’t fully consider the effect of pedestrian initial position and anthropometry, while these factors were found to have great effect on head response. These limitations could affect the accuracy of the generated head impact conditions.

Finally, but most importantly, the simplified MB human models and vehicle models cannot be used to further study the potential problem posed above: whether better performing vehicles in current sub-system procedure will obtain lower head injury risk in realistic full-scale pedestrian impact. To answer this question and find the reasons behind it, the effect of those factors on head response, such as vehicle pre-deformation caused by torso, head-neck interaction, head rotational kinematics, and head impact geometry need to be accurately modeled and understood.

\section{Finite Element Modeling}

Recently, the pedestrian finite element models (PFEM) were developed, validated, and widely used to study the pedestrian biomechanics. Compared to MB models, FE models consist of deformable elements and can be used to predict injury based on tissue level criteria by calculating variables such as stress, strain, and strain rate. The FE method allows for modelling of complex geometries and using advanced material laws, and provides a high level of detail. 
With FE models, the load path through the human body during an impact can be quantified at the tissue level.

Maeno and Hasegawa (2001) developed a finite element human model, known as the Total Human Model for Safety (THUMS) (Figure 2.16), and demonstrated its application in pedestrian impact simulation. This model is a commercially available full-body FE model developed jointly by Toyota Motor Corporation and Toyota Central R \& D Labs Inc (Watanabe et al. 2012; TMC 2011). Other existing full-body pedestrian FE models are the simplified pedestrian GHBMC models (Untaroiu 2015), the pedestrian HUMOS2-model developed during the EC-funded HUMOS1 and HUMOS2 projects (Vezin and Verriest 2005), and the JAMA pedestrian model (Sugimoto and Yamazaki 2005).

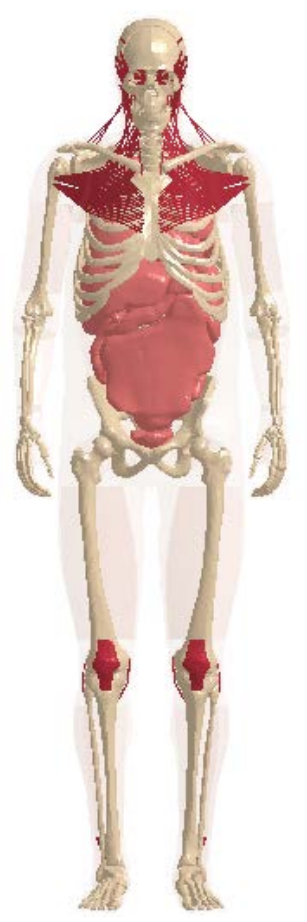

Figure 2.16 THUMS AM50 Pedestrian Model

In these PFEMs, the THUMS model is most widely used to investigate the biomechanics in vehicle-pedestrian impact (Snedeker et al. 2003; Watanabe et al. 2012; Han et al. 2012; Chen 
et al. 2015). The main releases of THUMS that have so far been made available are version 1 , launched in the year 2000, version 3, launched in 2006, and the latest, version 4, launched in 2010. In comparison with earlier versions of THUMS, version 4 has a refined mesh, includes internal organs and more solid-element muscles that provide damping in an impact. The THUMS pedestrian version 4 has been developed using new computed tomography (CT) scans although the head model of version 3 was re-used with a refined mesh (TMC 2011).

The PFEM together with detailed vehicle FE model provided ideal tools to further investigate the pedestrian biomechanics, including the head impact conditions, head injury risk, and the vehicle passive safety assessment. Also, these models can be used to study whether better performing vehicles in current sub-system procedure will obtain lower head injury risk in realistic full-scale pedestrian impact, and made it possible to study the complex effect of those factors on head response, including vehicle pre-deformation caused by torso, head-neck interaction, head rotational kinematics, and head impact geometry.

However, as more accurate, detailed, and complex models are expected to be used to predict injury at tissue level, the PFEM must be validated both in terms of global kinematics and the local response on kinetics and injury prediction at tissue level.

Previous evaluations of THUMS PFEM have been mainly focused on the component level, and generally demonstrates biofidelic responses (Maeno et al. 2001; Iwamoto et al. 2002; TMC 2011; Poulard et al. 2015), though THUMS v4.0 spine appeared to be stiffer than the volunteers’ during shoulder impact (Pass et al. 2015).

The response of the human body to vehicle impact has been extensively studied using PMHS (Snedeker et al. 2005, Kerrigan et al. 2007; Subit et al. 2008; Paas et al. 2015; Forman et al. 2015), providing primary source data for the development and validation of PFEM. Since the 
strain is used as failure criteria for PFEM, the evaluation of PFEM to ensure proper quantification of strain distribution is very important. However, early studies have rarely monitored bone strains during pedestrian impact tests (Snedeker et al. 2005). Also, during the impact with the vehicle, the PMHS biomechanical response was found to be greatly influenced by its anthropometry (Subit et al. 2008; Paas et al. 2012). Consequently, evaluating PFEM biofidelity using PMHS is challenging as the difference in anthropometry between PMHS and PFEM could limit the model's capability to accurately capture cadaveric responses. Watanabe et al. (2012) and Paas et al. (2015) evaluated THUMS (v4.0) against PMHS test data, but their approaches, global geometrical scaling of PFEM (scale on PMHS stature and weight) didn’t consider the local differences in anthropometry such as pelvic height and knee height, which could reduce the model's capability to reproduce the experimental kinematics. Recently, Poulard et al. (2016) used geometric personalization via morphing to modify THUMS (v4.01) geometry to match the specific PMHS anatomy used by Subit et al. (2008), and the morphed THUMS has been shown to better predict cadaveric trajectories than globally scaled THUMS, highlighting the need for geometrical personalization. However, these model evaluations focused on subject trajectories and no analysis was performed on the other sets of reported data; velocity, accelerations, strains, and injuries.

Finally, the subjects used in the PMHS tests are generally very old (Paas et al. 2016; Subit et al. 2008; Kerrigan et al. 2005), and these aged subjects might have lower bone density, which might change their bone material properties and injury threshold (Watanabe et al. 2012). In addition, many injuries were observed in the tests (Subit et al. 2008), while previous model evaluation studies did not enable these tissue failures in the simulation (Chen et al.2017; Paas et 
al. 2016), so the effect of the tissue failure on the following pedestrian response needs to be verified.

Therefore, further evaluation on the THUMS biofidelity is needed on the segmental velocity, accelerations, local strains, and injuries, with full consideration of the anthropometry dependence of pedestrian response in the model evaluation. Furthermore, the effect of tissue failure during impact needs to be verified.

\subsubsection{Countermeasures}

Basically, passive safety countermeasures to protect the pedestrian's head are to reduce vehicle stiffness and increase deformation space. Previous studies focused on the vehicle design improvement towards better performance in the sub-system test procedure. Kerkeling et al. (2005) discussed the hood design and hood hinge concept for pedestrian protection, including designing the hood inner panel with a more uniform stiffness distribution. Based on investigations by simulation and physical impact tests, they came to the conclusion that two options will work for an inlaid hood with a sheet metal design: reduced gages for a steel hood, or aluminum hood. For the hood mountings like hood hinge, the suggestion was made to design with deformable parts and a collapse mechanism with low vertical stiffness. Similarly, Wanke et al. (2005) proposed to increase hood height to ensure deformation space, using a thin steel hood, deformable hood hinge, and deformable multi-part plastic cowl system. Kalliske and Friesen, (2001) replaced the conventional $0.75 \mathrm{~mm}$ metal outer panel with a sheet of $0.50 \mathrm{~mm}$ while the reinforced structure was not changed. They found this change can reduce the stiffness of the bonnet to obtain better performance in head impactor tests. Liu et al. (2009) presented a sandwich hood design that has 
the potential to improve the hood's ability to absorb the impact energy of a pedestrian's head with a relatively small underhood clearance.

Mizuno et al. (2001) reported that the A-pillar produces remarkably high HIC values due to its excessive rigidity in modern vehicles. The HIC value of 1,000 is associated with a dynamic deformation of $76 \mathrm{~mm}$ for the windshield area. Thus, in order to limit the HIC below 1,000, a dynamic deformation greater than $76 \mathrm{~mm}$ is thought to be necessary (Mizuno et al. 2001). However, it is unfeasible to ensure such a deformation in the A-pillar area while maintaining enough structure strength in frontal and roll-over collisions. Therefore, a pair of A-pillar airbags (Figure 2.17) has been introduced by Autoliv R\&D to enhance the safety performance for pedestrian head protection.
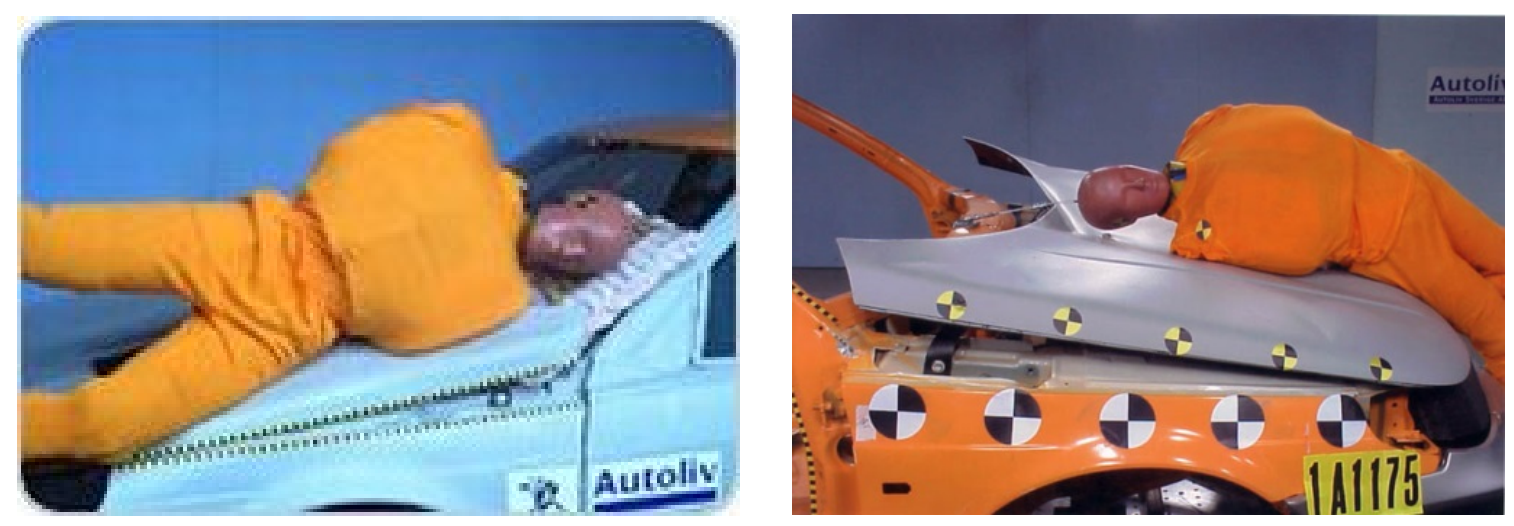

Figure 2.17 Pedestrian airbag and pop-up hood system (Fredriksson et al., 2001)

Fredriksson et al. (2001) developed a protection system, pop-up hood system, to decrease the severity of head-to-bonnet impacts. The system is enabled at the impact by a sensor located in the bumper, and was tested by a headform impacting the bonnet at various locations and speeds up to $50 \mathrm{~km} / \mathrm{h}$, as well as with a complete car front on a sled impacting a pedestrian dummy. In headform tests at $40 \mathrm{~km} / \mathrm{h}$, the system decreased the HIC values to acceptable levels $(<1000)$ in all test points for the lifting bonnet, including the headform contact locations above 
where the bonnet was lifted. In the $50 \mathrm{~km} / \mathrm{h}$ headform test above the bonnet's stiffest point, a large reduction of the HIC value was achieved. Also, Nagatomi et al. (2005) conducted full-scale dummy tests to study the effect of the pop-up hood on injury parameters and kinematics using the POLAR pedestrian dummy, and confirmed the effectiveness of the pop-up hood system by the significant reduction of HIC values in impact tests.

There are also many active safety technologies developed for pedestrian protection, including pedestrian detection by sensor, auto-brake system, and so on. Recently, more studies have focused on the integration of active safety and passive safety technology. Fredriksson et al. (2011) conducted a parameter study using finite element models of pedestrian dummy and generic passenger vehicles to study the potential of active, passive, and integrated (combined active and passive) safety systems in reducing pedestrian upper body loading in typical impact configurations. Three countermeasures were modeled: an active autonomous braking system, a passive deployable countermeasure including a deployable hood, lifting $100 \mathrm{~mm}$ in the rear, and a lower windshield air bag, and an integrated system combining the active and passive systems. This study found both the active (autonomous braking) and passive deployable system studied had a potential to decrease pedestrian upper body loading. An integrated pedestrian safety system combining the active and passive systems increased the potential of the individual systems in reducing pedestrian head and chest loading.

\subsection{Summary of Background}

The literature survey reviewed the pedestrian accidents, pedestrian head injury biomechanics, method and tools to study pedestrian injury, current sub-system test procedure, and those countermeasures including passive and active safety technologies. 
One of the most notable characteristics in the field of pedestrian passive safety is the regulation and evaluation method of the sub-system test, instead of full-scale impact. Although the debate and critique on the limitations of simplified sub-system test procedure has lasted for decades (Kerrigan et al. 2008; Hardy et al. 2007), it is still selected as the only testing method to evaluate vehicle aggressiveness due to its major advantages, including repeatability, ease of use, and low cost. Consequently, the industry is focusing on optimizing their vehicle designs towards this component test procedure, instead of the realistic full-scale pedestrian impact. This poses an important question regarding how accurately and to what degree the sub-system test procedure can represent the full-scale impact.

To the best of the author's knowledge, no comprehensive study has been performed to answer this question, partly due to the limited power of the methods and tools previously used. The cadaver test cannot serve this purpose because they are not repeatable, and each subject has different intrinsic conditions which made the parametric study not feasible. The dummy test cannot do this either, because testing the various vehicle designs involves many tests at a huge cost, and the anthropometry available on the physical dummy is very limited. Finally, the multibody models cannot accurately model the vehicle deformation, head-neck interaction, and head impact geometry.

The FE human model and vehicle model provide high accuracy and enough complexity to answer this question, but before this model can be used its biofidelity needs to be further evaluated. Therefore, the literature survey has identified a need for answering the following three questions:

Question 1 - How much can the current sub-system test represent the full-scale pedestrian impact? 
Question 2 - Why does or does not the sub-system test accurately capture the pedestrian head response in full-scale impact?

Question 3 - Is the THUMS PFEM biofidelic to capture the pedestrian response in fullscale impact? 


\section{Chapter 3 Biofidelity Evaluation of Geometrically Personalized}

\section{PFEM against PMHS Test Data}

Previous literature reviews have identified the need to further evaluate the THUMS PFEM biofidelity before using it to investigate the pedestrian head response in full-scale impact. In this chapter, the THUMS PFEM were geometrically personalized and then comprehensively evaluated against four cadaver tests results. Also, the effect of tissue failure during impact on pedestrian kinematics and injury risk was verified.

\subsection{Introduction}

Pedestrian fatalities each year worldwide highlights the inherent need to understand pedestrian injuries associated with vehicle-pedestrian impact. Epidemiology has shown that the head, lower extremity, thorax, and pelvis are the most common pedestrian injury regions, and their injury sources are mainly windshield, bumper, hood, and bonnet leading edge (BLE) (Chen et al. 2009).

Pedestrian finite element models (PFEM) offer some promising advantages as advanced injury prediction tools in vehicle-pedestrian impacts: detailed anatomical information, improved material modes and injury prediction at the tissue level. To be considered valid (within the assumptions and limitations of the model), the ability of the PFEM to predict biofidelic response and injury needs to be evaluated during the development of the model.

One of the existing PFEMs is the Total Human Model for Safety (THUMS) pedestrian model (TMC 2011), which has been widely used to investigate the biomechanics in vehicle- 
pedestrian impact (Snedeker et al. 2003; Watanabe et al. 2012; Han et al. 2012; Chen et al. 2015). Previous evaluations of THUMS PFEM have been mainly focused on the component level, and generally demonstrates biofidelic responses (Maeno et al. 2001; Iwamoto et al. 2002; TMC 2011; Poulard et al. 2015), though THUMS v4.0 spine appeared to be stiffer than the volunteers' during shoulder impact (Paas et al. 2015).

The response of the human body to vehicle impact has been extensively studied using PMHS (Snedeker et al. 2005, Kerrigan et al. 2007; Subit et al 2008; Paas et al. 2015; Forman et al. 2015), providing primary source data for the development and validation of PFEM. Since the strain is used as failure criteria for PFEM, the evaluation of PFEM to ensure proper quantification of strain distribution is very important. However, early studies have rarely monitored bone strains during pedestrian impact tests (Snedeker et al. 2005). Recently, four PMHS were subjected to $40 \mathrm{~km} / \mathrm{h}$ full-scale pedestrian impact tests using a mid-size sedan buck (Subit et al. 2008). Digital and laser scanning devices characterized the subject and the environment and extensive measurements were taken during the impact, including segmental kinematics and local strains in the bony structures. These methodological advances permit greater description of boundary conditions allowing modelers to properly evaluate a PFEM.

During the impact with the vehicle, the PMHS biomechanical response was found to be greatly influenced by its anthropometry (Subit et al. 2008; Paas et al. 2012). Consequently, evaluating PFEM biofidelity using PMHS is challenging as the difference in anthropometry between PMHS and PFEM could limit the model's capability to accurately capture cadaveric responses. Watanabe et al. (2012) and Paas et al. (2015) evaluated THUMS (v4.0) against PMHS test data, but their approaches, global geometrical scaling of PFEM (scale on PMHS stature and weight) didn't consider the local differences in anthropometry such as pelvic height and knee 
height, which could reduce the model's capability to reproduce the experimental kinematics. Recently, Poulard et al. (2016) used geometric personalization via morphing to modify THUMS (v4.01) geometry to match the specific PMHS anatomy used by Subit et al. (2008), and the morphed THUMS has been shown to better predict cadaveric trajectories than globally scaled THUMS highlighting the need for geometrical personalization. However, these model evaluations focused on subject trajectories and no analysis was performed on the others sets of reported data: velocity, accelerations, strains, and injuries.

Finally, the subjects used in the PMHS tests are generally very old (Paas et al. 2016; Subit et al. 2008; Kerrigan et al. 2005), and these aged subjects might have lower bone density, which might change their bone material properties and injury threshold (Watanabe et al. 2012). Also, many injuries were observed in the tests (Subit et al. 2008), while previous studies did not enable these tissue failures in the simulation (Chen et al.2017; Paas et al. 2016), so the effect of the tissue failure on the following pedestrian response needs to be verified.

The goal of this study was to comprehensively evaluate the capability of geometrically personalized THUMS PFEM capturing cadaveric response in full-scale vehicle-pedestrian impact, and study the effect of the tissue failure on the following pedestrian response.

\subsection{Evaluation of Morphed Models Against Mid-Size Sedan}

\subsubsection{Method}

\section{$\underline{\text { Test data }}$}

Two male PMHS of different anthropometries (PMHS 1: 154 cm, 72.6 kg; PMHS 2: 183 cm, $114 \mathrm{~kg}$ ) were impacted laterally by a mid-size sedan buck traveling at $40 \mathrm{~km} / \mathrm{h}$. All subjects were in a walking posture with the left foot ahead and the right foot behind the body, and the 
arms bounded together at the wrists anterior to the thorax/abdomen. The postures were acquired from pre-test photographs and measurements of landmark heights and distances.

The details about vehicle profile, subjects' anthropometry, instrumentation, data processing, and injury report were published in previous literatures (Untaroiu et al. 2007; Subit et al. 2008; Poulard et al. 2016) and are included in the Appendix 3.1-3.4.

Two-dimensional kinematic trajectories were obtained for the head, spine, pelvis, and the lower extremities using high-speed videos. Trajectories were assessed relative to the vehicle in a vehicle coordinate system (VCS) (Figure 3.1, Figure 3.2). Additional subject instrumentation included six-axis accelerometers located in the posterior head, spine, pelvis, femur and ankle. The subjects were also instrumented with four strain gages glued to the mid-shaft cross section of the struck-side (right) tibia and the femora bilaterally (Untaroiu et al. 2007). The gauge locations are listed in Appendix 3.3. Detailed autopsy reports on injuries are listed in Appendix

\section{4 .}

\section{Morphed pedestrian models and setup}

The AM50 THUMS model was first positioned to match the posture of each PMHS using body angles converted from the experimental measurement. Two geometrically personalized PFEMs were created via morphing to the anthropometric specifications of the PMHS. The morphing procedure was based on a Dual Kriging interpolation process using 144 control points constructed from external anthropometric measurements taken from CT scans (Poulard et al. 2016). The morphed models were previously evaluated using eleven component-level loading cases relevant for the biomechanics of pedestrian impact and the models were deemed biofidelic (Poulard et al. 2015), and were then positioned according to the measurements acquired prior to 
impact (Figure 3.1). Detailed anthropometry parameters including body region height and mass distribution are listed in Appendix 3.1.
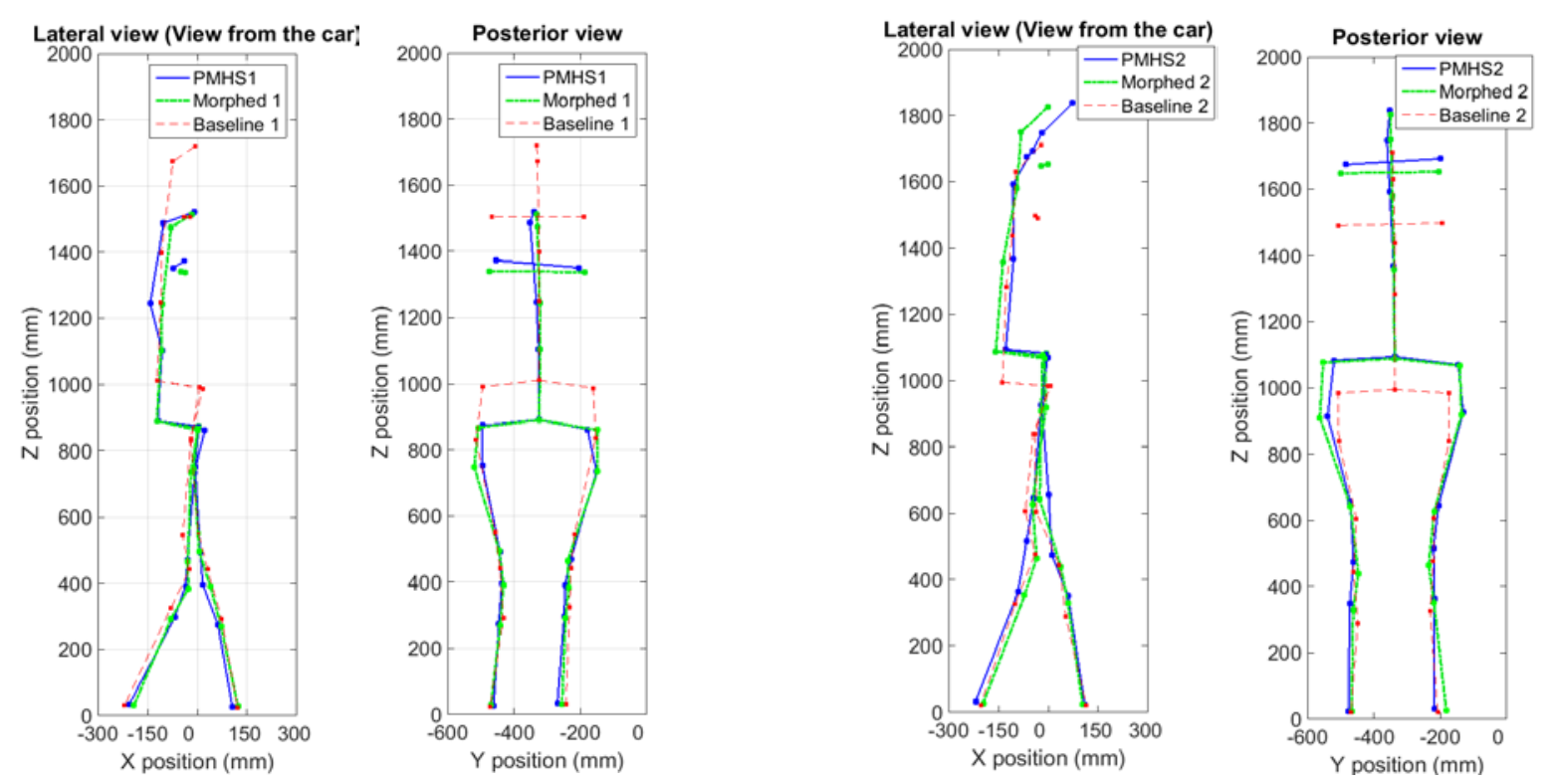

Figure 3.1 Initial positions. Lateral and posterior view of subject 1 (left) and subject 2 (right)

Baseline and morphed models were integrated with a validated FE model (Watanabe et al. 2012) of the mid-size sedan buck used in Subit et al. (2008) (Figure 3.2). The front-end structures included engine hood, upper/lower grill, bumper assembly, and other structures relevant to pedestrian impact. The vehicle model was subjected to the prescribed velocity history obtained in each test. The friction coefficient between the HBM and the vehicle was 0.5, and 0.6 between the feet and ground (Chen et al. 2009, Chen et al. 2015; Watanabe et al. 2012).

The simulations were carried out with LS-Dyna R7.1.1 (LSTC, Livermore, CA, USA), and hosted on a 4-node cluster (Dual Opteron 6238, 12 cores/node, 64 GB/node). The pre- and post-processing was done in LS-PREPOST (v4.1, LSTC, Livermore, CA, USA), and scripts were written in Matlab (R2012b, The MathWorks Inc., Natick, MA, USA). 

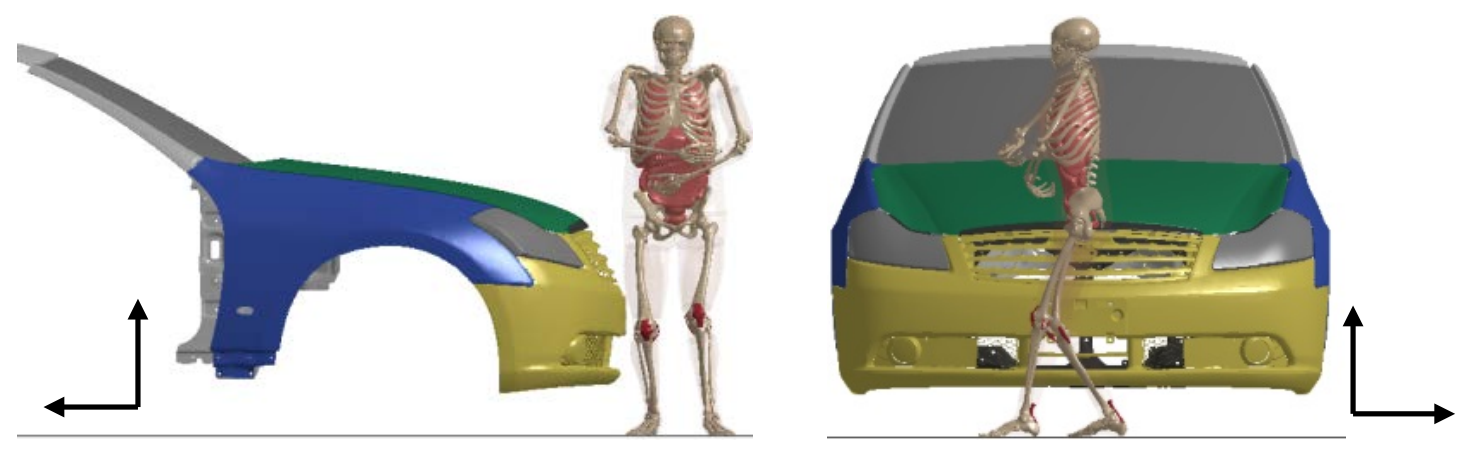

Figure 3.2 Simulation set up (Morphed model 1)

\section{Model instrumentation}

The trajectories of the head, spine, pelvis, right knee (KneeR), and right heel (HeelR) were assessed in the vehicle coordinate system (VCS). Triaxial accelerations were measured by derivation of the velocity measured on a node attached to the center of the bony location (head, T1/T2, T8/T9, L5, pelvis, femur, and ankle). To be compared with test results, model strains were measured by calculating the distances of the external nodes of the corresponding elements on the cortical bones.

Simulation data was computed at $1000 \mathrm{~Hz}$ for displacement and velocity and $10 \mathrm{KHz}$ for acceleration and strain and digitally filtered with a CFC180 SAE channel class filter as it was in the experiments.

\section{Quantitative assessment of the model response}

The CORrelation and Analysis (CORA) method version 3.6.1 (Gehre et al. 2009) was applied to compare the simulation and experimental curves. The end time of the evaluation interval for kinematics was 20 ms after the time of the first PMHS head-vehicle contact (101 ms for subject 1 and $145 \mathrm{~ms}$ for subject 2), while 100 ms was set for lower extremity strain. Since the evaluation is based on a direct comparison, the corridor rating was ignored and the weighting factors of the sub-scores was equally 0.333 for the phase, size and shape. Ratings for each body 
region were combined into an overall rating using mean value, and the student test was used to determine which sets of CORA sub-scores were significantly different from each other.

\section{Injury Prediction}

Injury was predicted deterministically based on strain threshold, and probabilistically using injury risk functions, and compared with the injuries reported in the necropsy. No element elimination was enabled, and the simulation end time for injury predictions was 20 ms after the time of the first head-vehicle contact.

Using the Probabilistic method (PM), injury criteria extracted from the literature were used to assess the risk of sustaining specific injuries (Table 3.1). Injury risk functions are listed in Appendix 3.5.

Table 3.1 Injury criteria

\begin{tabular}{|c|c|c|c|}
\hline Injury criteria & Predicted injury & Injury severity & Reference \\
\hline $\mathrm{HIC}$ & Skull fracture & $\begin{array}{l}\text { Moderate } \\
\text { (AIS2+) }\end{array}$ & Kuppa 2004 \\
\hline Chest deflection (lateral, frontal) & Rib fracture & Serious (AIS3+) & $\begin{array}{l}\text { Kuppa et al. 2003, } \\
\text { Kent et al. } 2005\end{array}$ \\
\hline Clavicle axial compressive force & Clavicle fracture & AIS2+ & Zhang et al. 2013 \\
\hline Pelvis deflection & Pelvis fracture & AIS2+ & Gunji et al. 2012 \\
\hline $\begin{array}{l}\text { Thigh moment (proximal, middle, } \\
\text { distal) }\end{array}$ & Femur fracture & AIS3+ & Kerrigan et al. 2004 \\
\hline Leg moment (middle) & $\begin{array}{l}\text { Tibia/Fibula } \\
\text { fracture }\end{array}$ & AIS2+ & Kerrigan et al. 2004 \\
\hline Upper tibia axial force & $\begin{array}{l}\text { Tibia plateau } \\
\text { fracture }\end{array}$ & AIS2+ & $\begin{array}{l}\text { Banglmaier et al. } \\
1999\end{array}$ \\
\hline $\begin{array}{l}\text { Knee bending angle and shear } \\
\text { displacement }\end{array}$ & $\begin{array}{l}\text { Knee } \quad \text { ligament } \\
\text { rupture }\end{array}$ & AIS2+ & $\begin{array}{l}\text { Ivarsson et al. 2004, } \\
\text { Mo et al. } 2014\end{array}$ \\
\hline $\begin{array}{l}\text { Ankle bending angle and lower tibia } \\
\text { axial load }\end{array}$ & Ankle injury & AIS2+ & Funk et al. 2002 \\
\hline
\end{tabular}

Using Deterministic method (DM), the maximum principal strain from post-processing was used to predict the cortical bone fractures and ligament failures. The thresholds were set to 
represent a population similar to the PMHS in terms of age (62 years old for both subjects) using values extracted from the literature.

Table 3.2 Strain threshold used in the deterministic method

\begin{tabular}{llll}
\hline Predicted injury & Body region & Threshold & Reference \\
\hline $\begin{array}{l}\text { Cortical bone fracture (skull, rib, scapula,clavicle, } \\
\text { vertebrae, femur, tibia/fibula, calcaneus,talus) }\end{array}$ & $\begin{array}{l}\text { Head, thorax, } \\
\text { shoulder, spine, thigh, }\end{array}$ & 1.5\% & $\begin{array}{l}\text { Yasuki et al. 2010; } \\
\text { Golman et al. 2014 }\end{array}$ \\
$\begin{array}{l}\text { Cortical bone fracture (pelvis) } \\
\text { Cruciate ligament rupture (ACL, PCL) }\end{array}$ & Pelvis & $1.0 \%$ & Snedeker et al. 2003 \\
Collateral ligament rupture (MCL, LCL) & Knee & $24 \%$ & Mo et al. 2014 \\
Capsular ligament rupture (CL) & Knee & $28 \%$ & Mo et al. 2014 \\
Other ligament rupture (ALL, PLL, LF, ISL) & Spine & $151 \%$ & Quinn et al. 2007 \\
Ankle ligaments rupture (ATFL, PTFL, CFL) & Foot & $50 \%$ & Bass et al. 2007; \\
\hline
\end{tabular}

\subsubsection{Results}

Full pedestrian impact sequences are available in Figure 3.3 and Figure 3.4. For PMHS 1 and morphed 1, the bumper contacted the right knee and the distal femur, and the lower bumper contacted the right lower leg (Figure 3.3, $0 \mathrm{~ms}$ ), so the right tibia and femur accelerated rapidly and reached peak value around $10 \mathrm{~ms}$ (Figure 3.7). At $15 \mathrm{~ms}$, the bumper contacted the left lower extremity and the left tibia and femur accelerated and reached peak value at 20 ms. The medial aspect of the right femur and right tibia was then placed in tension while the lateral aspect was placed in compression (Figure 3.33). On the contrary, for the left lower extremity, the lateral aspect was placed in tension while the medial aspect was placed in compression. The pelvis of the PMHS and morphed model impacted the BLE at 30 ms (Figure3.3, $30 \mathrm{~ms}$ ), while the pelvis of the baseline model went over the hood delaying its impact with the vehicle, and obtained lower pelvis peak velocity (10.2 m/s for baseline; $12.4 \mathrm{~m} / \mathrm{s}$ for morphed). As the vehicle continued, the pelvis and lower spine motion gradually induced lateral bending in the thoracic 
spine. The morphed model exhibited less lateral bending on the spine (Figure 3.3 and Figure 3.30, 60ms) and the neck (Figure 3.30, 80ms; Figure 3.3, 90ms). Due to the difference in pelvic impact location, the baseline model exhibited a delay in spine motion and caused the thoracic and cervical spine to remain straight longer than the morphed model (Figure 3.3, $60 \mathrm{~ms}$ ), which resulted in a lower velocity of the upper body (head, T1 and T8) (Figure 3.5), and then delayed lateral bending of the neck, and reduced head rotation (Figure 3.3, $90 \mathrm{~ms}$ ). Finally, morphed models displayed head impacting on the middle hood as in the experiments, while the head of the baseline model impacted on the cowl area (Figure 3.3, $120 \mathrm{~ms}$ ). Quantitatively, the morphed model captured better head impact timing (105 ms) than the baseline model (129 ms) with respect to PMHS 1 (101 ms).

For subject 2, lesser differences between the kinematics of the models were observed. Due to the higher pelvis than the BLE, the pelvis of all models slid over the hood (Figure 3.4, 40 ms). Again, the morphed model exhibited fewer laterals bending on the spine and the neck (Figure 3.4 and Figure 3.30, 80-120 ms), suggesting the slightly stiffer spine of THUMS than the PMHSs. With $72 \mathrm{~mm}$ lower pelvis $(1023 \mathrm{~mm})$ than PMHS and morphed model, the baseline model obtained higher pelvis velocity and acceleration (Figure 3.6 and Figure 3.8), and higher spine lateral bending and head rotation (Figure 3.4, 80-120 ms). Finally, the head of the morphed model (Morphed 2) impacted the windshield as in the experiments (PMHS 2), while the head of the baseline model (Baseline 2) impacted the cowl area (Figure 3.4, $160 \mathrm{~ms}$ ). Quantitatively, the morphed model captured better head impact timing (145 ms) than the baseline model (131 ms) with respect to the PMHS 2 (146 ms). 

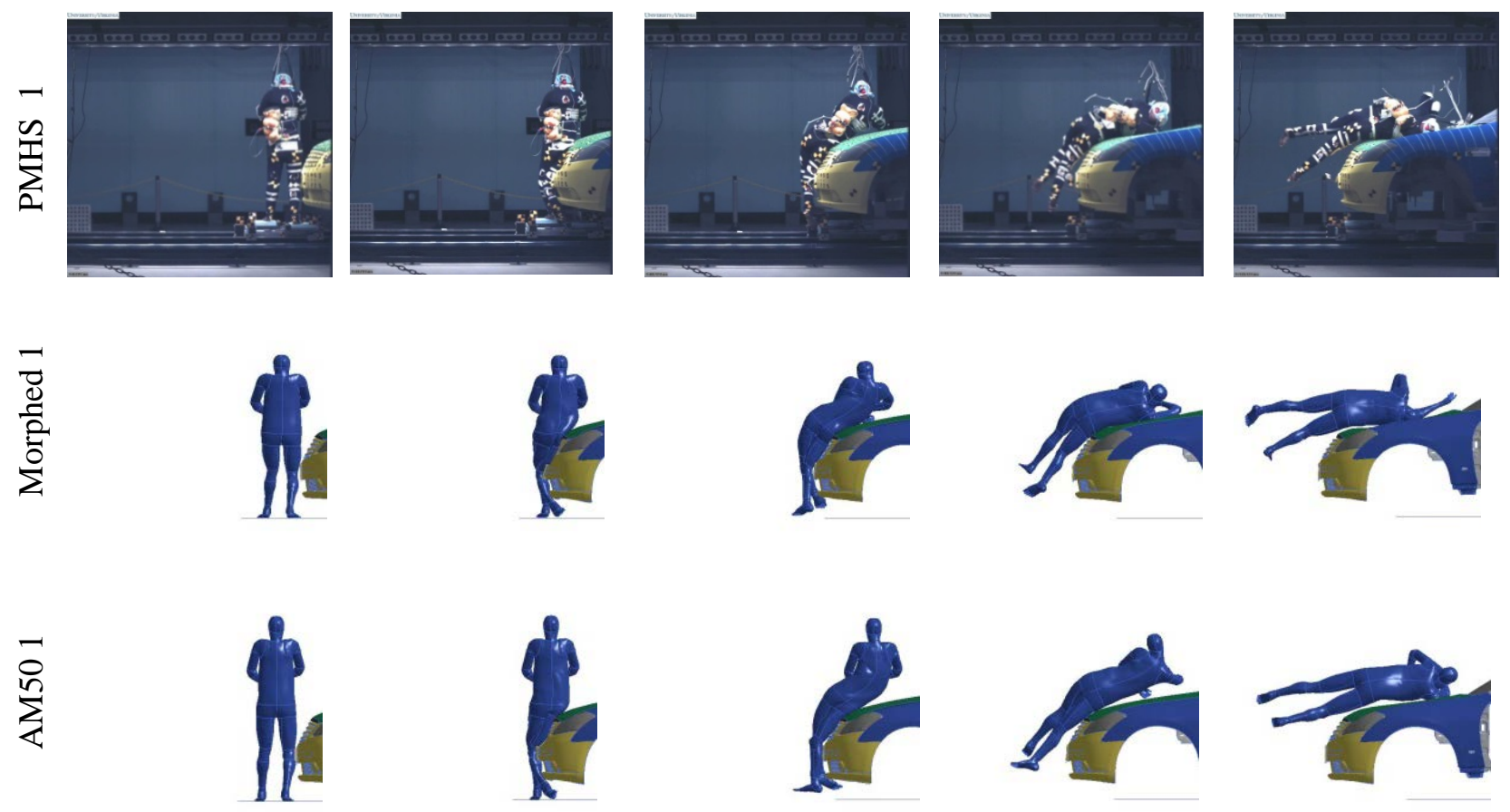

$0 \mathrm{~ms}$

$30 \mathrm{~ms}$

$60 \mathrm{~ms}$

$90 \mathrm{~ms}$

$120 \mathrm{~ms}$

Figure 3.3 Pedestrian impact sequences of PMHS and models (subject 1).
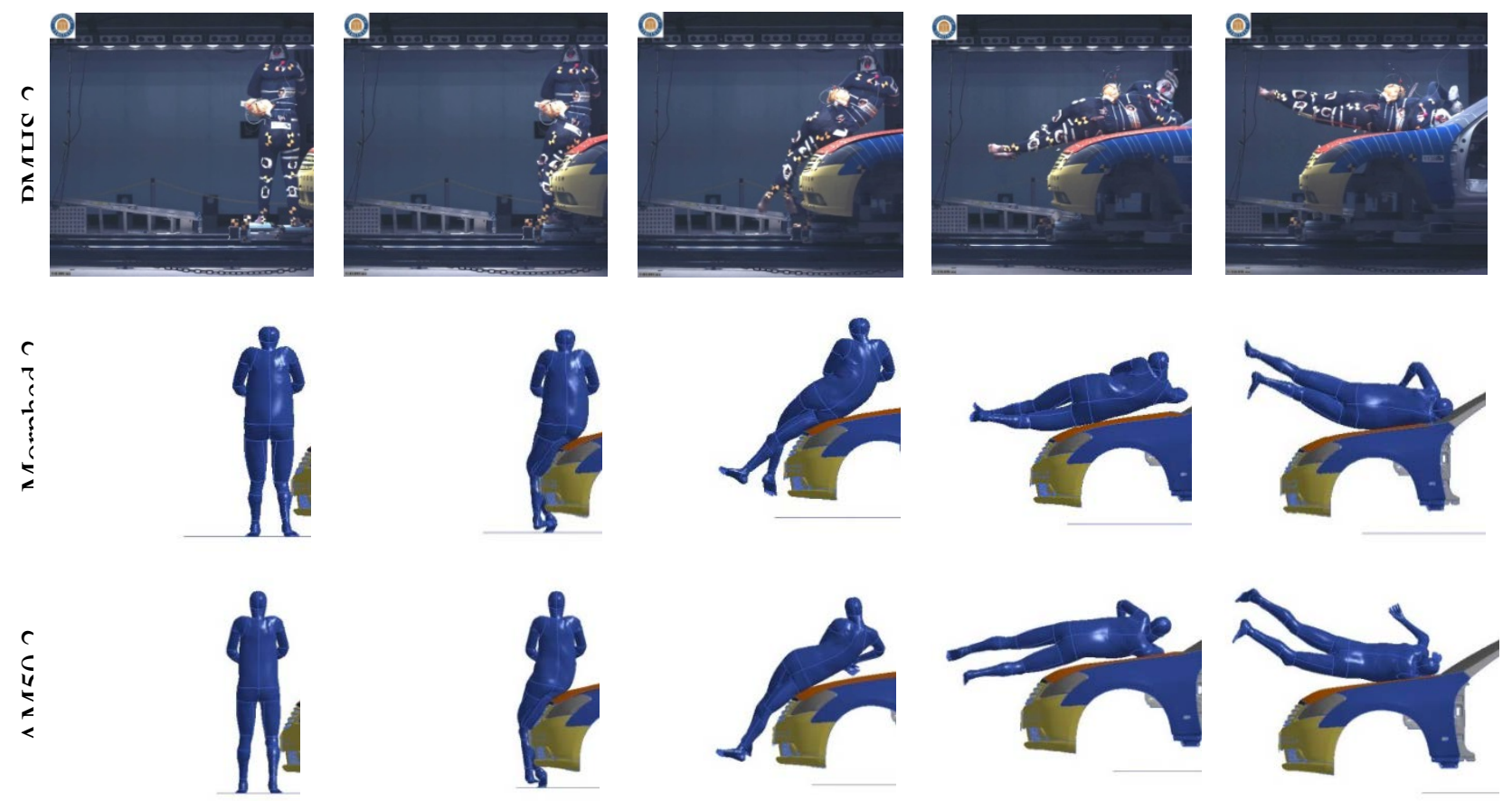

$0 \mathrm{~ms}$

$40 \mathrm{~ms}$

$80 \mathrm{~ms}$

$120 \mathrm{~ms}$

$160 \mathrm{~ms}$

Figure 3.4 Pedestrian impact sequences of PMHS and models (subject 2). 
The morphed models obtained higher CORA scores than the baseline models (morphed 1: 0.974, baseline 1: 0.886; morphed 2: 0.975 , baseline 2: 0.944 , see Table 3 ) on time history of body resultant velocities with respect to test results (Figure 3.5 - Figure 3.6). Significant differences in CORA sub-scores were observed between morphed and baseline models for the size (difference=0.158, $\mathrm{p}<0.001$, see Table 8).

The resultant acceleration time histories predicted by the morphed models were similar to the acceleration observed in the experiments $(\mathrm{CORA}=0.862)$ (Figure 3.7 - Figure 3.8), while baseline models showed less consistent time histories and lower average CORA score (0.822).

The strain time histories predicted by the morphed models were similar to those observed in the experiments with regards to the magnitude though the difference was observed with regards to timing (Figure 3.34 - Figure 3.34), while baseline models showed inconsistent magnitudes and lower CORA scores $(0.659 \pm 0.005)$ than morphed models $(0.704 \pm 0.069)$.

Table 3.3 Model evaluation results using the CORA method

\begin{tabular}{|c|c|c|c|c|c|c|c|c|}
\hline \multirow[b]{2}{*}{$\begin{array}{l}\text { Physical } \\
\text { value }\end{array}$} & \multicolumn{4}{|c|}{ Morphed 1} & \multicolumn{4}{|c|}{ Baseline 1} \\
\hline & $\begin{array}{l}\text { Cross } \\
\text { correlation }\end{array}$ & Size & $\begin{array}{l}\text { Phase } \\
\text { shift }\end{array}$ & $\begin{array}{l}\text { Total } \\
\text { rating }\end{array}$ & $\begin{array}{l}\text { Cross } \\
\text { correlation }\end{array}$ & Size & $\begin{array}{l}\text { Phase } \\
\text { shift }\end{array}$ & $\begin{array}{l}\text { Total } \\
\text { rating }\end{array}$ \\
\hline Displacement & 0.995 & 0.866 & 0.997 & 0.953 & 0.982 & 0.662 & 0.917 & 0.854 \\
\hline Velocity & 0.991 & 0.930 & 1.000 & 0.974 & 0.979 & 0.743 & 0.936 & 0.886 \\
\hline Acceleration & 0.935 & 0.743 & 0.876 & 0.851 & 0.929 & 0.739 & 0.939 & 0.869 \\
\hline Strain & 0.873 & 0.390 & 0.701 & 0.655 & 0.867 & 0.367 & 0.731 & 0.655 \\
\hline $\begin{array}{c}\begin{array}{c}\text { Physical } \\
\text { value }\end{array} \\
\end{array}$ & \multicolumn{4}{|c|}{ Morphed 2} & \multicolumn{4}{|c|}{ Baseline 2} \\
\hline Displacement & 0.978 & 0.781 & 0.942 & 0.901 & 0.996 & 0.866 & 0.991 & 0.951 \\
\hline Velocity & 0.992 & 0.967 & 0.967 & 0.975 & 0.992 & 0.838 & 1.000 & 0.944 \\
\hline Acceleration & 0.939 & 0.690 & 0.987 & 0.872 & 0.937 & 0.478 & 0.911 & 0.775 \\
\hline Strain & 0.866 & 0.463 & 0.931 & 0.753 & 0.831 & 0.317 & 0.841 & 0.663 \\
\hline
\end{tabular}


Table 3.4 CORA ratings (Subject 1)

\begin{tabular}{|c|c|c|c|c|c|c|c|c|c|}
\hline \multirow[b]{2}{*}{ Value } & \multirow[b]{2}{*}{ Location } & \multicolumn{4}{|c|}{ Morphed model 1} & \multicolumn{4}{|c|}{ Baseline model 1} \\
\hline & & $\begin{array}{c}\text { Cross } \\
\text { correlation }\end{array}$ & Size & $\begin{array}{c}\text { Phase } \\
\text { shift }\end{array}$ & $\begin{array}{c}\text { Total } \\
\text { rating }\end{array}$ & $\begin{array}{c}\text { Cross } \\
\text { correlation }\end{array}$ & Size & $\begin{array}{c}\text { Phase } \\
\text { shift }\end{array}$ & $\begin{array}{l}\text { Total } \\
\text { rating }\end{array}$ \\
\hline \multirow{12}{*}{ 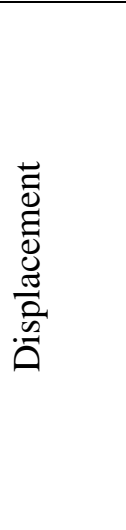 } & Head Y & 1.000 & 0.896 & 1.000 & 0.965 & 0.999 & 0.985 & 1.000 & 0.995 \\
\hline & Head Z & 0.999 & 0.966 & 1.000 & 0.988 & 0.995 & 0.470 & 1.000 & 0.822 \\
\hline & T1 Y & 1.000 & 0.975 & 1.000 & 0.992 & 0.999 & 0.766 & 1.000 & 0.922 \\
\hline & $\mathrm{T} 1 \mathrm{Z}$ & 0.998 & 0.889 & 1.000 & 0.962 & 0.988 & 0.851 & 1.000 & 0.946 \\
\hline & T8 Y & 1.000 & 0.925 & 1.000 & 0.975 & 0.998 & 0.629 & 1.000 & 0.876 \\
\hline & T8 Z & 0.996 & 0.921 & 1.000 & 0.973 & 0.985 & 0.534 & 1.000 & 0.840 \\
\hline & Pelvis Y & 1.000 & 0.952 & 1.000 & 0.984 & 0.998 & 0.558 & 1.000 & 0.852 \\
\hline & Pelvis Z & 0.994 & 0.659 & 1.000 & 0.885 & 0.964 & 0.211 & 1.000 & 0.725 \\
\hline & Knee R Y & 0.970 & 0.605 & 0.961 & 0.845 & 0.874 & 0.543 & 0.000 & 0.473 \\
\hline & Knee R Z & 0.998 & 0.887 & 1.000 & 0.962 & 0.998 & 0.851 & 1.000 & 0.949 \\
\hline & Heel R Y & 0.989 & 0.753 & 1.000 & 0.914 & 0.993 & 0.820 & 1.000 & 0.938 \\
\hline & Heel R Z & 0.996 & 0.965 & 1.000 & 0.987 & 0.994 & 0.731 & 1.000 & 0.908 \\
\hline \multirow{6}{*}{$\begin{array}{l}\stackrel{D}{0} \\
\frac{0}{0} \\
>\end{array}$} & Head & 0.997 & 0.891 & 1.000 & 0.962 & 0.978 & 0.650 & 1.000 & 0.876 \\
\hline & $\mathrm{T} 1$ & 0.990 & 0.896 & 1.000 & 0.962 & 0.984 & 0.707 & 1.000 & 0.897 \\
\hline & T8 & 0.980 & 0.961 & 1.000 & 0.980 & 0.953 & 0.599 & 1.000 & 0.850 \\
\hline & Pelvis & 0.987 & 0.959 & 1.000 & 0.982 & 0.972 & 0.750 & 0.618 & 0.780 \\
\hline & Knee R & 0.997 & 0.991 & 1.000 & 0.996 & 0.997 & 0.902 & 1.000 & 0.966 \\
\hline & Heel R & 0.993 & 0.883 & 1.000 & 0.958 & 0.992 & 0.848 & 1.000 & 0.947 \\
\hline \multirow{9}{*}{ 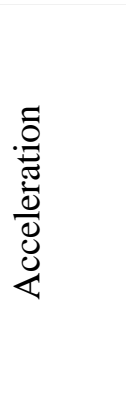 } & Head & 0.971 & 0.950 & 0.806 & 0.909 & 0.922 & 0.129 & 0.975 & 0.675 \\
\hline & $\mathrm{T} 1$ & 0.901 & 0.563 & 0.382 & 0.615 & 0.880 & 0.962 & 0.836 & 0.893 \\
\hline & Pelvis & 0.932 & 0.710 & 0.970 & 0.871 & 0.933 & 0.733 & 1.000 & 0.889 \\
\hline & Femur R & 0.893 & 0.674 & 1.000 & 0.856 & 0.928 & 0.725 & 0.936 & 0.863 \\
\hline & Femur L & 0.966 & 0.669 & 1.000 & 0.878 & 0.953 & 0.743 & 1.000 & 0.899 \\
\hline & Tibia L & 0.914 & 0.789 & 0.810 & 0.838 & 0.917 & 0.885 & 0.706 & 0.836 \\
\hline & Tibia R & 0.963 & 0.701 & 1.000 & 0.888 & 0.951 & 0.724 & 1.000 & 0.892 \\
\hline & Ankle L & 0.940 & 0.636 & 0.949 & 0.842 & 0.959 & 0.781 & 1.000 & 0.913 \\
\hline & Ankle R & 0.932 & 0.997 & 0.970 & 0.967 & 0.919 & 0.970 & 1.000 & 0.963 \\
\hline \multirow{7}{*}{ 急 } & LF 5 & 0.935 & 0.254 & 0.716 & 0.635 & 0.977 & 0.717 & 0.892 & 0.862 \\
\hline & LF 6 & 0.950 & 0.810 & 0.737 & 0.832 & 0.985 & 0.113 & 0.753 & 0.617 \\
\hline & LF 7 & 0.920 & 0.532 & 0.680 & 0.711 & 0.986 & 0.345 & 0.753 & 0.695 \\
\hline & LF 8 & 0.681 & 0.211 & 0.308 & 0.400 & 0.527 & 0.040 & 0.043 & 0.203 \\
\hline & RT 9 & 0.898 & 0.623 & 0.869 & 0.797 & 0.948 & 1.000 & 0.851 & 0.933 \\
\hline & RT 10 & 0.919 & 0.255 & 0.945 & 0.707 & 0.905 & 0.302 & 0.957 & 0.721 \\
\hline & RT 11 & 0.807 & 0.046 & 0.649 & 0.501 & 0.744 & 0.054 & 0.865 & 0.554 \\
\hline
\end{tabular}


Table 3.5 CORA ratings (Subject 2)

\begin{tabular}{|c|c|c|c|c|c|c|c|c|c|}
\hline \multirow[b]{2}{*}{ Value } & \multirow[b]{2}{*}{ Location } & \multicolumn{4}{|c|}{ Morphed model 2} & \multicolumn{4}{|c|}{ Baseline model 2} \\
\hline & & $\begin{array}{c}\text { Cross } \\
\text { correlation }\end{array}$ & Size & $\begin{array}{l}\text { Phase } \\
\text { shift }\end{array}$ & $\begin{array}{l}\text { Total } \\
\text { rating }\end{array}$ & $\begin{array}{c}\text { Cross } \\
\text { correlation }\end{array}$ & Size & $\begin{array}{c}\text { Phase } \\
\text { shift }\end{array}$ & $\begin{array}{c}\text { Total } \\
\text { rating }\end{array}$ \\
\hline \multirow{12}{*}{ 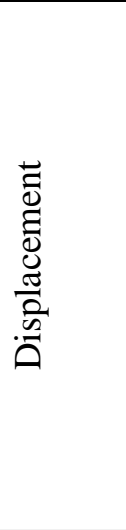 } & Head Y & 1.000 & 0.927 & 1.000 & 0.976 & 0.999 & 0.829 & 1.000 & 0.943 \\
\hline & Head Z & 0.998 & 0.950 & 1.000 & 0.983 & 0.995 & 0.888 & 1.000 & 0.961 \\
\hline & $\mathrm{T} 1 \mathrm{Y}$ & 1.000 & 0.943 & 1.000 & 0.981 & 1.000 & 0.894 & 1.000 & 0.965 \\
\hline & $\mathrm{T} 1 \mathrm{Z}$ & 0.996 & 0.901 & 1.000 & 0.966 & 0.997 & 0.826 & 1.000 & 0.941 \\
\hline & T8 Y & 1.000 & 0.813 & 1.000 & 0.937 & 1.000 & 0.988 & 1.000 & 0.996 \\
\hline & T8 Z & 0.992 & 0.384 & 1.000 & 0.792 & 0.995 & 0.938 & 1.000 & 0.978 \\
\hline & Pelvis Y & 0.999 & 0.815 & 1.000 & 0.938 & 1.000 & 0.882 & 1.000 & 0.961 \\
\hline & Pelvis Z & 0.764 & 0.633 & 0.308 & 0.568 & 0.985 & 0.570 & 1.000 & 0.852 \\
\hline & Knee R Y & 0.998 & 0.620 & 1.000 & 0.873 & 0.995 & 0.757 & 1.000 & 0.917 \\
\hline & Knee R Z & 1.000 & 0.728 & 1.000 & 0.909 & 0.999 & 0.942 & 1.000 & 0.980 \\
\hline & Heel R Y & 0.997 & 0.882 & 1.000 & 0.960 & 0.990 & 0.937 & 0.892 & 0.940 \\
\hline & Heel R Z & 0.999 & 0.777 & 1.000 & 0.926 & 0.997 & 0.935 & 1.000 & 0.978 \\
\hline \multirow{6}{*}{$\frac{\stackrel{D}{0}}{\frac{0}{0}}$} & Head & 0.996 & 0.992 & 1.000 & 0.996 & 0.995 & 0.741 & 1.000 & 0.912 \\
\hline & $\mathrm{T} 1$ & 0.987 & 0.980 & 1.000 & 0.989 & 0.983 & 0.839 & 1.000 & 0.941 \\
\hline & T8 & 0.994 & 0.965 & 1.000 & 0.986 & 0.992 & 0.732 & 1.000 & 0.908 \\
\hline & Pelvis & 0.981 & 0.919 & 0.802 & 0.901 & 0.993 & 0.828 & 1.000 & 0.940 \\
\hline & Knee R & 0.998 & 0.998 & 1.000 & 0.999 & 0.998 & 0.988 & 1.000 & 0.995 \\
\hline & Heel R & 0.995 & 0.950 & 1.000 & 0.981 & 0.993 & 0.901 & 1.000 & 0.965 \\
\hline \multirow{9}{*}{ 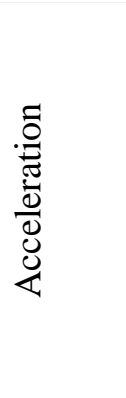 } & Head & 0.987 & 0.712 & 0.994 & 0.898 & 0.915 & 0.327 & 0.526 & 0.589 \\
\hline & $\mathrm{T} 1$ & 0.906 & 0.777 & 1.000 & 0.894 & 0.950 & 0.376 & 0.890 & 0.739 \\
\hline & Pelvis & 0.894 & 0.854 & 0.885 & 0.878 & 0.879 & 0.318 & 0.790 & 0.662 \\
\hline & Femur R & 0.957 & 0.515 & 1.000 & 0.824 & 0.931 & 0.330 & 1.000 & 0.753 \\
\hline & Femur L & 0.959 & 0.434 & 1.000 & 0.798 & 0.959 & 0.346 & 1.000 & 0.768 \\
\hline & Tibia L & 0.956 & 0.634 & 1.000 & 0.863 & 0.966 & 0.538 & 1.000 & 0.835 \\
\hline & Tibia R & 0.944 & 0.743 & 1.000 & 0.896 & 0.940 & 0.605 & 0.994 & 0.846 \\
\hline & Ankle L & 0.920 & 0.753 & 1.000 & 0.891 & 0.950 & 0.592 & 1.000 & 0.847 \\
\hline & Ankle R & 0.928 & 0.789 & 1.000 & 0.906 & 0.940 & 0.873 & 1.000 & 0.938 \\
\hline \multirow{7}{*}{ 急 } & LF 5 & 0.933 & 0.299 & 0.812 & 0.681 & 0.897 & 0.106 & 0.833 & 0.612 \\
\hline & LF 6 & 0.689 & 0.340 & 0.884 & 0.638 & 0.518 & 0.172 & 0.006 & 0.232 \\
\hline & LF 7 & 0.928 & 0.927 & 0.937 & 0.931 & 0.909 & 0.432 & 0.982 & 0.774 \\
\hline & LF 8 & 0.748 & 0.243 & 0.871 & 0.621 & 0.804 & 0.402 & 0.986 & 0.730 \\
\hline & RT 9 & 0.899 & 0.502 & 1.000 & 0.801 & 0.863 & 0.100 & 0.992 & 0.651 \\
\hline & RT 10 & 0.953 & 0.665 & 0.947 & 0.855 & 0.952 & 0.341 & 0.931 & 0.741 \\
\hline & RT 11 & 0.867 & 0.067 & 1.000 & 0.645 & 0.788 & 0.014 & 1.000 & 0.601 \\
\hline
\end{tabular}

Table 3.6 Difference of CORA sub-scores between morphed models and baseline models

\begin{tabular}{cccc}
\hline Difference (p value) & Cross correlation & Size & Phase shift \\
\hline Displacement & $-0.002(0.83)$ & $0.060(0.24)$ & $0.016(0.76)$ \\
Velocity & $0.005(0.1)$ & $0.158(0.00)$ & $0.015(0.67)$ \\
Acceleration & $0.004(0.57)$ & $0.108(0.12)$ & $0.006(0.88)$ \\
Strain & $0.021(0.28)$ & $0.089(0.31)$ & $0.034(0.61)$ \\
\hline
\end{tabular}



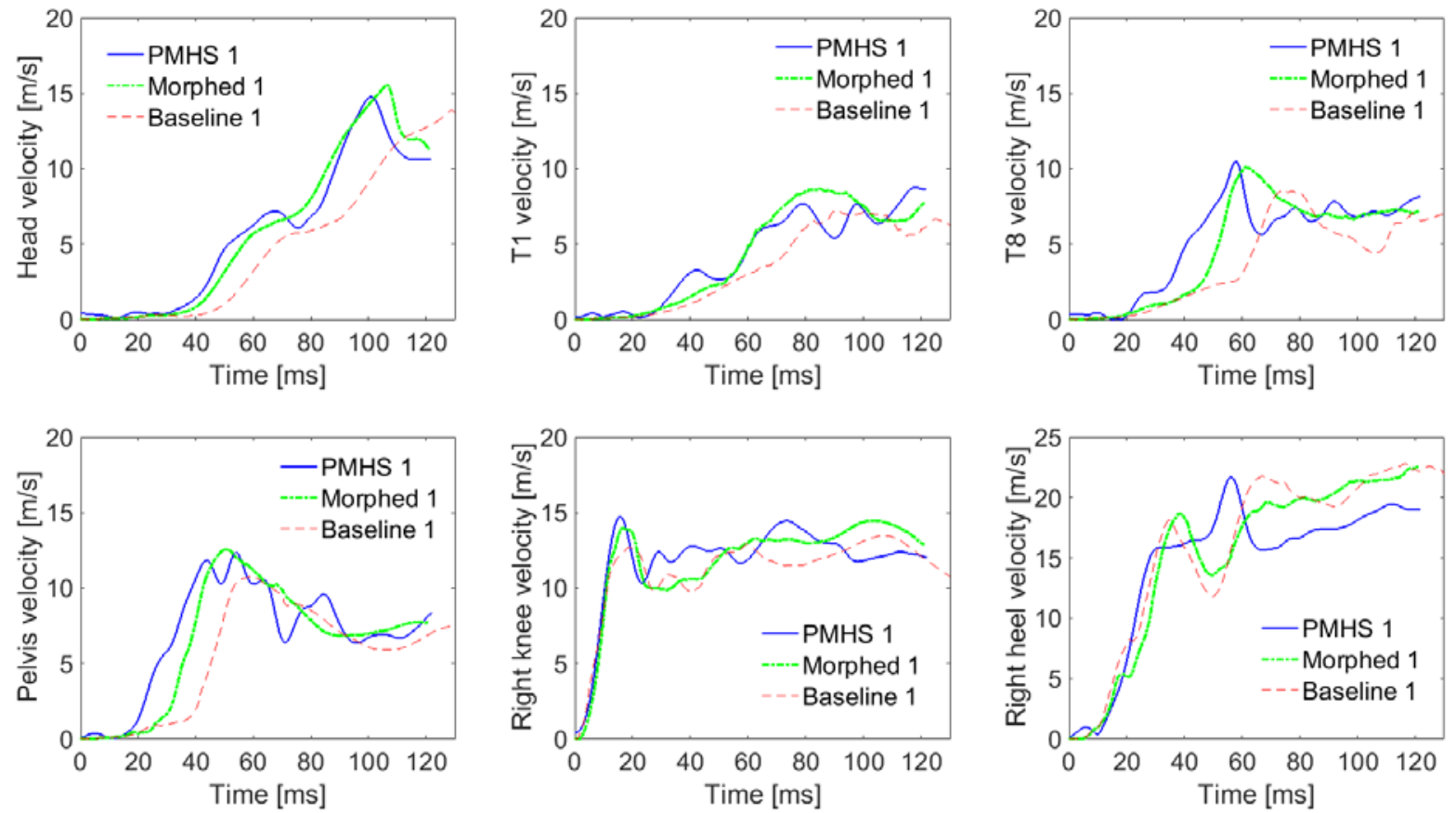

Figure 3.5 Time history of resultant velocity on each body region (subject 1)
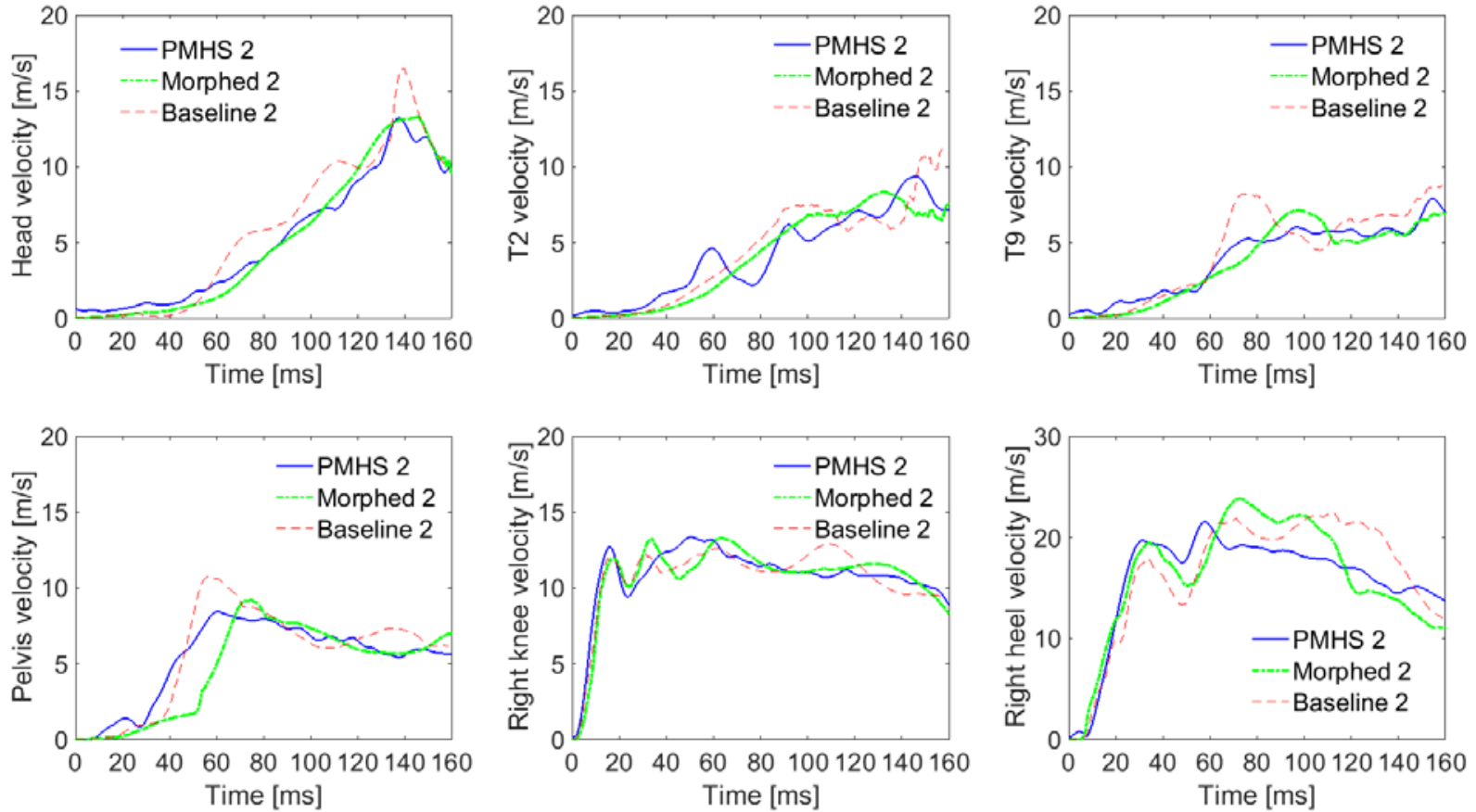

Figure 3.6 Time history of resultant velocity on each body region (subject 2) 

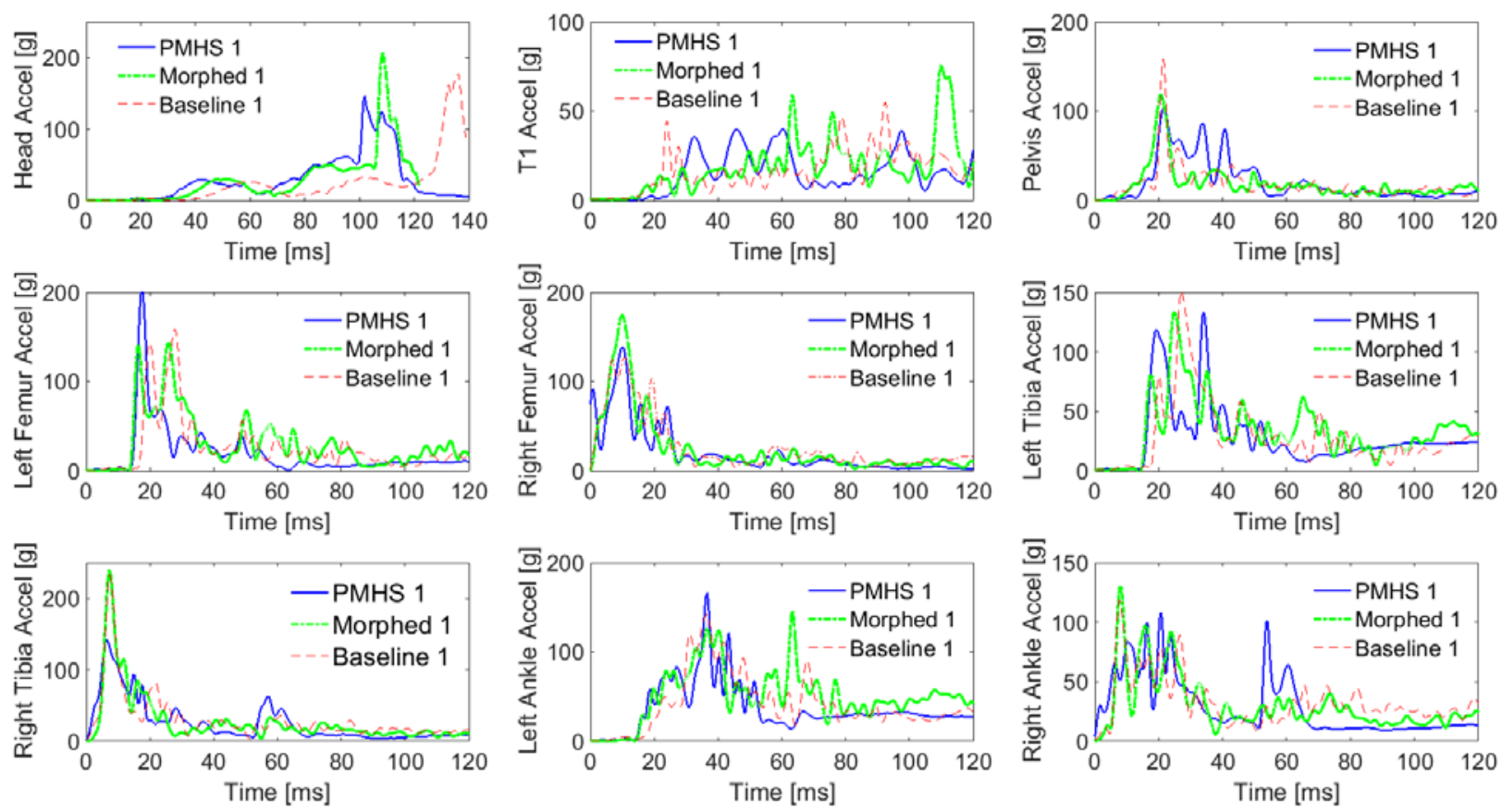

Figure 3.7 Time history of resultant acceleration on each body region (subject 1)
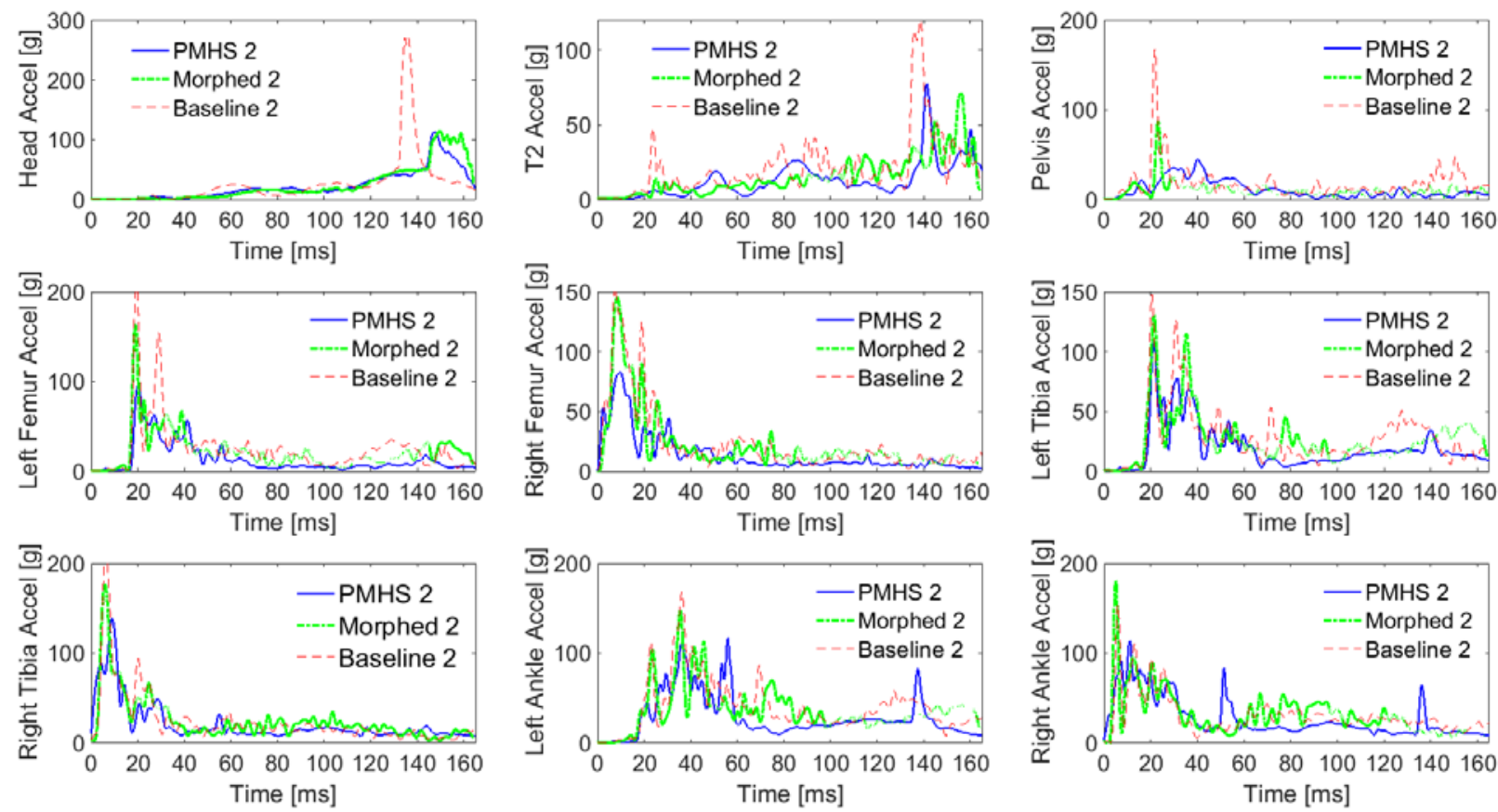

Figure 3.8 Time history of acceleration on each body region (subject 2) 
$\underline{\text { Injury }}$

Morphed models captured injury risks for most of the body regions (Table 3.7 - Table 3.8, and Figure 3.9 - Figure 3.10), while baseline models made different predictions especially for the head, thorax, and pelvis.

Table 3.7 Summary of predicted injury for PMHS 1, morphed model (Morphed 1) and baseline model (Baseline 1) using probabilistic method (PM) and deterministic method (DM) (Inconsistent model predictions with PMHS are bolded and underlined).

\begin{tabular}{|c|c|c|c|c|c|c|}
\hline \multirow{2}{*}{ Body region } & \multirow{2}{*}{ Predicted injury } & \multirow{2}{*}{ PMHS 1} & \multicolumn{2}{|c|}{ Morphed 1 } & \multicolumn{2}{|c|}{ Baseline 1} \\
\hline & & & PM & DM & $\mathbf{P M}$ & DM \\
\hline Head & Skull Fx. & No & $74.9 \%$ (AIS2+) & No & $80.8 \%$ (AIS2+) & No \\
\hline Neck (soft) & Ligament Rup. & C6-C7 all lig. Rup. & Not eva. & All Lig. Rup. & Not eva. & All Lig. Rup. \\
\hline Neck (bone) & Vertebrae Fx. & C6 SpProc.Fx. & Not eva. & No & Not eva. & No \\
\hline Thorax R & Rib Fx. & No & $1.1 \%(\mathrm{AIS3}+)$ & No & $11.8 \%(\mathrm{AIS3}+$ ) & $\frac{N o}{\text { No }}$ \\
\hline Thorax L & Rib Fx. & Rib 6 Fx. & $10.3 \%(\mathrm{AIS3}+$ ) & Rib 7 Fx. & $1.1 \%(\mathrm{AIS} 3+)$ & No \\
\hline Thorax M & Sternum Fx. & No & Not eva. & No & Not eva. & No \\
\hline Shoulder R & Clav/scap Fx. & No & $0 \%(\mathrm{AIS} 2+$ ) & Scap Fx. & $0 \%(\mathrm{AIS} 2+$ ) & Scap Fx. \\
\hline Shoulder L & Clav/scap fx. & No & $0 \%(\mathrm{AIS} 2+)$ & No & $0 \%(\mathrm{AIS} 2+)$ & No \\
\hline Spine (soft) & Ligament Rup. & T11-12 PLL torn & Not eva. & All Lig. Rup. & Not eva. & No \\
\hline Spine (bone) & Vertebrae Fx. & T11 Endplate inf. Fx. & Not eva. & No & Not eva. & No \\
\hline Pelvis R & Pelvis Fx. & Mult .Lat. Fx. & \multirow{2}{*}{$78.8 \%(\mathrm{AIS} 2+)$} & Pubic bone Fx. & \multirow{2}{*}{$\underline{41 \%(A I S 2+)}$} & Acetabulum pubic bone \\
\hline Pelvis L & Pelvis Fx. & No & & Acetabulum Fx. & & No \\
\hline R Thigh & Femur Fx. & No & $0 \%(\mathrm{AIS3}+$ ) & No & $1.7 \%(\mathrm{AIS} 3+)$ & Femur \\
\hline L Thigh & Femur Fx. & No & $0.1 \%(\mathrm{AIS3}+$ ) & No & $4.3 \%(\mathrm{AIS} 3+)$ & Femur \\
\hline R Leg & Tibia/fibula Fx. & No & $1.5 \%(\mathrm{AIS} 2+)$ & No & $3.1 \%(\mathrm{AIS} 2+)$ & $\overline{\text { No }}$ \\
\hline L Leg & Tibia/fibula Fx. & No & $0.1 \%(\mathrm{AIS} 2+$ ) & Fib. Fx. & $6.2 \%(\mathrm{AIS} 2+)$ & Fib. Fx. \\
\hline R Knee (soft) & Ligament Rup. & MCL ACL Rup. & $100 \%(\mathrm{AIS} 2+)$ & MCL ACL Rup. & $100 \%(\mathrm{AIS} 2+)$ & MCL ACL Rup. \\
\hline R Knee (bone) & Tibia plat. Fx. & Tib.Plat. Fx. (Ant Lat) & $0.9 \%$ (AIS2+) & No & $1.1 \%$ (AIS2+) & No \\
\hline L Knee (soft) & Ligament Rup. & No & $100 \%(\mathrm{AIS} 2+)$ & LCL ACL_Rup. & $100 \%(\mathrm{AIS} 2+)$ & LCL Rup. \\
\hline L Knee (bone) & Tibia plat. Fx. & Tib.Plat Fx. (LCL) & $1.3 \%$ (AIS2+) & No & $1.3 \%$ (AIS2+) & No \\
\hline R Ankle (soft) & Ligament Rup. & No & \multirow{2}{*}{$0.1 \%(\mathrm{AIS} 2+$ ) } & No & \multirow{2}{*}{$0 \%(\mathrm{AIS} 2+$ ) } & CFL PTFL \\
\hline R Ankle(bone) & Calcaneus/Talus Fx. & No & & No & & $\frac{\text { No }}{\text { No }}$ \\
\hline L Ankle (soft) & Ligament Rup. & No & \multirow{2}{*}{$\underline{100 \%}$ (AIS2+) } & CFL ATFL Rup. & \multirow{2}{*}{$\underline{99.8 \%}$} & CFL ATFL PTFL RUp. \\
\hline L Ankle (bone) & Calcaneus/Talus Fx. & No & & No & & No \\
\hline
\end{tabular}


Table 3.8 Summary of predicted injury for PMHS 2, morphed model (Morphed 2) and baseline model (Baseline 2) using probabilistic method (PM) and deterministic method (DM).

\begin{tabular}{|c|c|c|c|c|c|c|}
\hline \multirow{2}{*}{ Body region } & \multirow{2}{*}{ Predicted injury } & \multirow{2}{*}{ PMHS 2} & \multicolumn{2}{|c|}{ Morphed 2} & \multicolumn{2}{|r|}{ Baseline 2} \\
\hline & & & $\mathbf{P M}$ & DM & PM & DM \\
\hline Head & Skull Fx. & No & $53.4 \%$ (AIS2+) & No & $95.7 \%$ (AIS2+) & Skull \\
\hline Neck (soft) & Ligament Rup. & No & Not eva. & All Lig. Rup. & Not eva. & All Lig. Rup. \\
\hline Neck (bone) & Vertebrae Fx. & No & Not eva. & No & Not eva. & $\mathrm{C} 1$ \\
\hline Thorax R & Rib Fx. & Rib 2-6 Fx. & 81\% (AIS3+) & Rib 3 Fx. & $\underline{7.5 \%}$ (AIS3+) & $\overline{\text { No }}$ \\
\hline Thorax L & Rib Fx. & Rib 4-7 Fx. & $47.8 \%(\mathrm{AIS3}+$ ) & Rib 3 Fx. & $0.1 \%$ (AIS3+) & No \\
\hline Thorax M & Sternum Fx. & Sternal Fx. & Not eva. & No & Not eva. & No \\
\hline Shoulder R & Clav/scap Fx. & No & $0 \%(\mathrm{AIS} 2+)$ & Clav. Fx. & $0 \%(\mathrm{AIS} 2+)$ & Scap. Fx. \\
\hline Shoulder L & Clav/scap fx. & Scap. Fx. & $0 \%(\mathrm{AIS} 2+$ ) & Scap. Fx. & $0 \%(\mathrm{AIS} 2+)$ & No \\
\hline Spine (soft) & Ligament Rup. & T11-12 all Lig. Rup. & Not eva. & ALL Rup. & Not eva. & $\overline{\text { No }}$ \\
\hline Spine (bone) & Vertebrae Fx. & T12 Sup Endplate Fx. & Not eva. & T3 Fx. & Not eva. & $\underline{\underline{\text { No }}}$ \\
\hline Pelvis R & Pelvis Fx. & Mult.Lat.Comp.Fx. & \multirow{2}{*}{$\underline{11.2 \%(\mathrm{AIS} 2+)}$} & Acetabulum Fx. & \multirow{2}{*}{$\underline{32.7 \%(\text { AIS2+) }}$} & Acetabulum pubic bone Fx. \\
\hline Pelvis L & Pelvis Fx. & No & & No & & No \\
\hline R Thigh & Femur Fx. & No & $\underline{91 \%(\mathrm{AIS3}+)}$ & No & $4.1 \%(\mathrm{AIS} 3+)$ & Femur \\
\hline L Thigh & Femur Fx. & No & $\overline{5.5 \%(\mathrm{AIS3}+)}$ & No & $2.9 \%(\mathrm{AIS} 3+$ ) & $\overline{\text { Femur }}$ \\
\hline R Leg & Tibia/fibula Fx. & No & $6.8 \%(\mathrm{AIS} 2+)$ & No & $3.1 \%(\mathrm{AIS} 2+)$ & No \\
\hline L Leg & Tibia/fibula Fx. & No & $0.3 \%(\mathrm{AIS} 2+$ ) & Fib. Fx. & $6 \%(\mathrm{AIS} 2+)$ & Fib. Fx. \\
\hline R Knee (soft) & Ligament Rup. & No & $100 \%$ (AIS2+) & MCL ACL Rup. & $100 \%$ (AIS2+) & MCL ACL Rup. \\
\hline R Knee (bone) & Tibia plat. Fx. & No & $0.2 \%(\mathrm{AIS} 2+)$ & No & $1.5 \%(\mathrm{AIS} 2+)$ & No \\
\hline L Knee (soft) & Ligament Rup. & LCL Rup. & $100 \%(\mathrm{AIS} 2+)$ & LCL $\underline{\text { ACL Rup. }}$ & $100 \%(\mathrm{AIS} 2+)$ & LCL Rup. \\
\hline L Knee (bone) & Tibia plat. Fx. & No & $0 \%(\mathrm{AIS} 2+)$ & No & $1.2 \%(\mathrm{AIS} 2+)$ & No \\
\hline R Ankle (soft) & Ligament Rup. & No & \multirow{2}{*}{$0 \%(\mathrm{AIS} 2+$ ) } & PTFL Rup. & \multirow{2}{*}{$8.9 \%(\mathrm{AIS} 2+$ ) } & PTFL CFL Rup. \\
\hline R Ankle(bone) & Calcaneus/Talus Fx. & No & & No & & No \\
\hline L Ankle (soft) & Ligament Rup. & ATF Lig. Fx. & \multirow{2}{*}{$100 \%(\mathrm{AIS} 2+$ ) } & CFL ATFL Rup. & \multirow{2}{*}{$99.2 \%(\mathrm{AIS} 2+$ ) } & CFL ATFL Rup. \\
\hline L Ankle (bone) & Calcaneus/Talus Fx. & No & & No & & No \\
\hline
\end{tabular}

For subject 1, using the probabilistic method (PM), morphed 1 predicted consistent HIC value while baseline 1 predicted different HIC value (morphed 1: 1938, baseline 1:2210, PMHS 1: 1730) due to different head kinematics and impact location. Low risk of severe (AIS3+) chest injury was predicted for both models (Morphed 1: 10\%, Baseline 1: 12\%) in accordance to the experiments (no severe chest injury reported). Morphed 1 predicted high risk of moderate pelvic injury (78\%) which is consistent with the report, but baseline 1 underestimated the moderate pelvis injury risk (41\%). Consistent with PMHS 1, very low long bone fracture risks (lower than $6 \%$ ) in lower extremities were predicted by both models. Both models captured high knee injury risks in tests, but overestimated the left ankle injury risk.

Using the deterministic method (DM), morphed 1 predicted one rib fracture as in experiment, with similar location of fracture (Morphed 1: left seventh rib, PMHS 1: left sixth rib), while no rib fracture was predicted by the baseline 1 . Both models predicted consistent pelvic 
fracture with the experiments, but morphed 1 predicted bilateral fracture while only right pelvic was reported. No femur or tibia shaft fracture was predicted by morphed model, which is consistent with the experiments, but baseline 1 predicted bilateral femur fractures. Both models captured the PMHS knee ligament ruptures. Avulsion fractures on tibia plateaus of PMHS 1 were not observed in simulation but high ligament strains (higher than 28\%) were observed at similar locations for both models. Both models overestimated the left ankle injury risk and predicted a left fibula fracture close to ankle impact location which was not reported in the experiments, and morphed 2 predicted right ankle rupture not reported in the experiments.

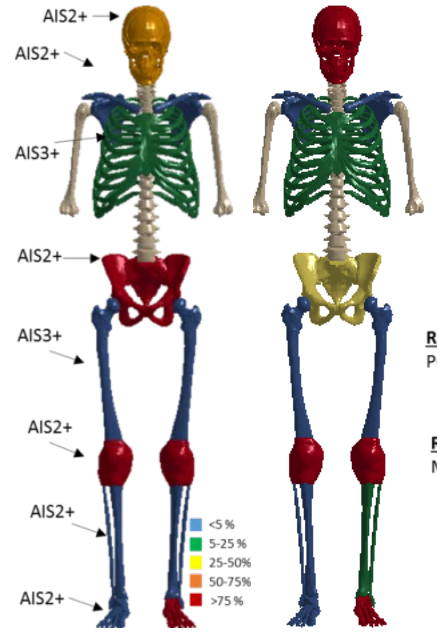

Morphed (PM) Baseline (PM)

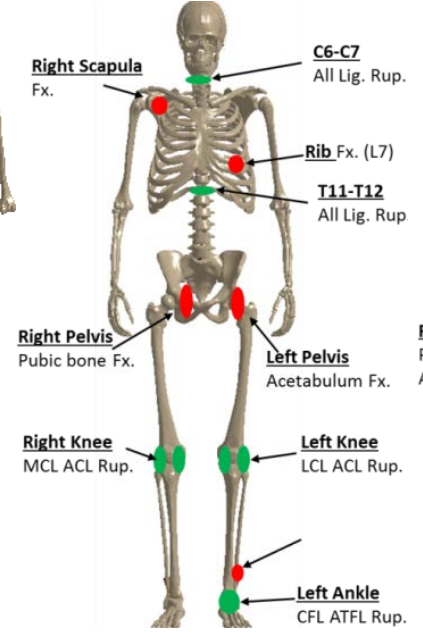

Morphed (DM)

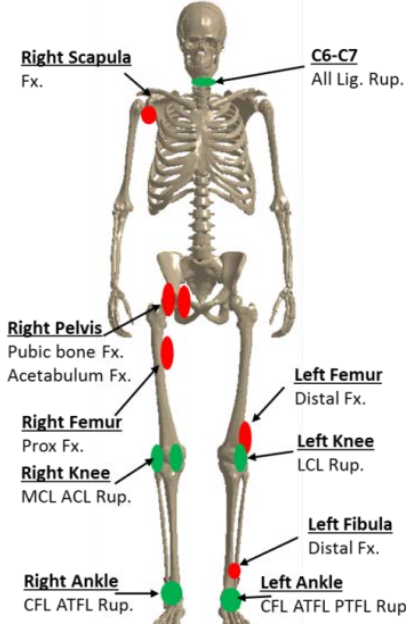

Baseline (DM)

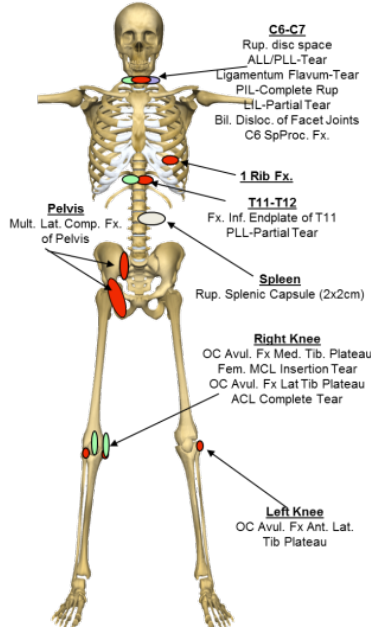

PMHS

Figure 3.9 Comparison of injury prediction of subject 1 for morphed model, baseline model, and PMHS injury from Subit et al. 2008

For subject 2, using the PM, morphed 2 predicted consistent HIC value while baseline 2 predicted different HIC value (morphed 2: 1136, baseline 2: 4530, PMHS 2: 924) due to different head kinematics and impact location (the head of baseline 2 impacted the windshield frame which is known to be the stiffest structure for pedestrian head impact). High risk (81\%) of severe (AIS3+) chest injury was predicted for morphed 2 in accordance with the experiments (9 
rib fractures), while the baseline model underestimated this risk at 8\%. Both models underestimated the risk of moderate pelvic injury (morphed 2: 11\%, Baseline 2: 33\%, PMHS 2: multiple lateral compression fracture). Both models generally predicted low risk of femur and tibia shaft fractures, which is consistent with test results, but morphed 1 overestimated the right femur injury risk. Both models captured high injury risks of the left knee in tests, but overestimated the injury risk of the right knee and left ankle.

Using the DM, morphed 2 did not predict but baseline 2 predicted the skull fracture which is not reported, mainly due to its stiffer head impact location. Both models underestimated the number of rib fractures (morphed 2: 2, baseline 2: 0, PMHS 2: 9). Morphed 2 captured a fracture of the left scapula as in the experiments but baseline 2 did not. Both models predicted pelvic fracture consistent with the experiments. No femur or tibia shaft fracture was predicted by morphed model, which is consistent with the experiments, but baseline 1 predicted bilateral femur fractures. Both models overestimated the PMHS right knee ligament ruptures. Comminuted and avulsion fracture on left fibula head and tibia plateau of PMHS 2 were not observed in simulation, but high ligament strains (higher than 28\%) were observed at similar locations for both models. Both models overestimated the ankle injury risks and predicted a left fibula fracture close to ankle impact location which was not reported in the experiments. 


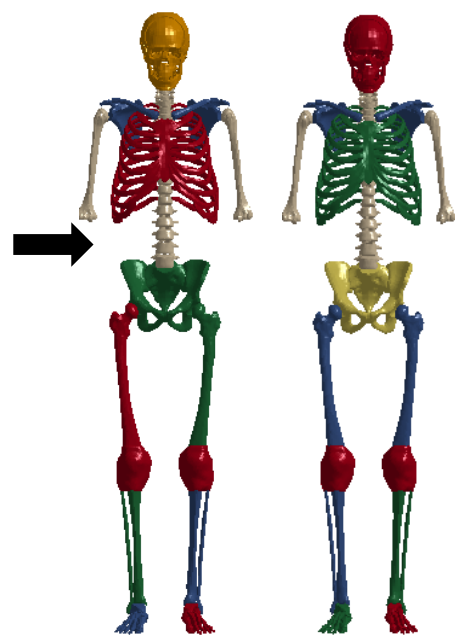

Morphed (PM) Baseline (PM)

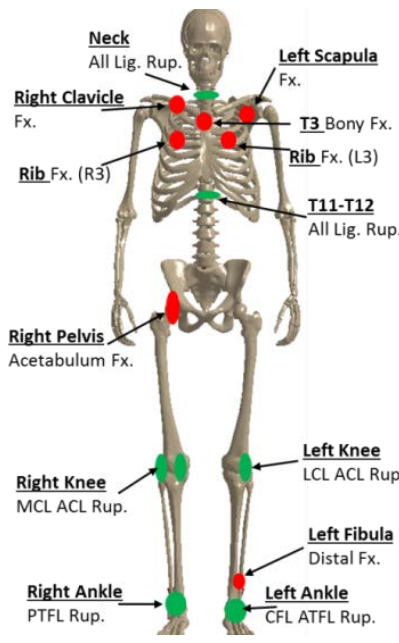

Morphed (DM)

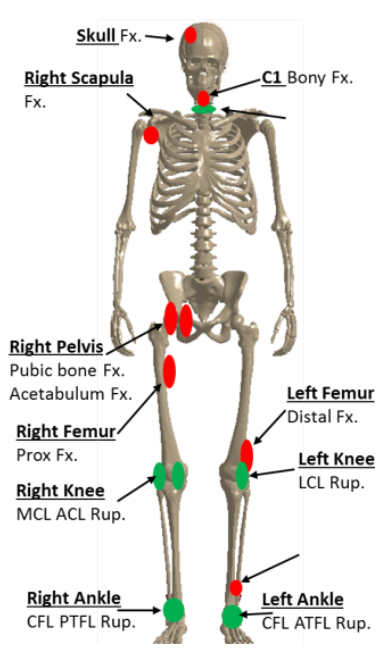

Baseline (DM)

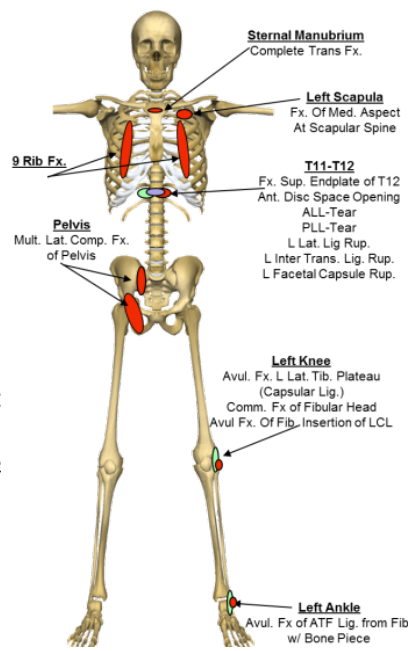

PMHS

Figure 3.10 Comparison of injury prediction of subject 2 for morphed model, baseline model, and PMHS injury from Subit et al. 2008

\subsubsection{Discussion}

The aim of this study was to evaluate the model capability of capturing the PMHS kinematics and injury risk. Injury prediction is a difficult task since it relies on not only the human model biofidelity and accuracy of the vehicle model, but also on the accuracy of the human model local anthropometry, initial positions, initial impact conditions, and the injury threshold, etc. Because some of these prerequisites were not satisfied, previous model evaluation works focused mainly on kinematics. Using the globally scaled models, the local anthropometry of the human model and accurate positioning cannot be guaranteed, which unavoidably reduced the model's capability to capture the kinematics. Since the trajectory decides the vehicle impact locations with varied local stiffness, and the impact velocity and angle caused by human rotation decide the impact energy, when the model kinematics are not close to those of the PMHS, its injury risk could cannot be fairly comparable. 
In this study, the model kinematics was evaluated and good matches were observed, including the trajectories and velocities of all body regions, and the magnitudes of simulated strains were found to be close to the test results. After these conditions were satisfied, the capabilities of the morphed models on capturing PMHS injuries were comprehensively evaluated.

\section{Evaluation of model response}

Watanabe et al. (2012) evaluated the model response against PMHS 2 using globally scaled THUMS 4.0 and the same sedan buck FE model as this study. With $71 \mathrm{~mm}$ higher initial global height, the globally scaled model observed a $73 \mathrm{~mm}$ shorter head excursion than did the cadaver test. In this study, the morphed model obtained a better matched pedestrian trajectory with the PMHS, especially on the head. With $6 \mathrm{~mm}$ lower initial global height than PMHS 2, the morphed model showed a $39 \mathrm{~mm}$ shorter head excursion than PMHS. Snedeker et al. (2005) found the early version THUMS 1.0 captured similar strains as the PMHS tests on cortical bone of lower extremities and pelvis, during full-scale impact with sedan and van. In this study, the morphed models received lower scores on strains than kinematics (Table 3.3 - Table 3.5), while time history curves showed consistent peak values with PMHS strains, which is important because the maximum strain was supposed to be used to predict injury risk.

Although any single metric is insufficient to provide a complete assessment of how well the simulated results match the experiments, the CORA method was found to provide the most comprehensive evaluation of the signal (Vavalle et al. 2013). Consequently, CORA scores were used to objectively evaluate the baseline and morphed models. The morphed PFEM responses showed good agreement in terms of shape and magnitude with the PMHS responses for the kinematics, accelerations and strains, with most CORA scores higher than 0.8 which was associated with a good fit between the model and the test. This choice of threshold 0.8 , while 
mostly arbitrary, was supported by previous studies (Vavalle et al. 2013, Poulard et al. 2014, Poulard et al. 2015). Interestingly, high CORA scores were also observed for the baseline models, especially on the displacement, despite simulation videos and time history curves showing fundamentally inconsistent trajectories and strain magnitudes with respect to test results (Figure 3.3 - Figure 3.8). Actually, the main problem of the baseline models is not in predicting PMHS displacement, but in capturing the trajectory (the positions of body regions in the vehicle coordinate system, see Figure 3.30) and velocity. Since the initial positions of those body regions (head, thorax, pelvis, etc.) are inconsistent with the PMHS, even given a perfect match displacement prediction, their final positions (impact locations on vehicle) will still be different from the PMHS. With the inconsistent velocity prediction (Figure 3.5 - Figure 3.6), the baseline models cannot predict the pedestrian impact conditions, and this makes them less able to predict injury, because incorrect vehicle impact locations cause different contact stiffness and inconsistent impact velocities cause different impact energy. Also, the student test showed the main differences in CORA sub-scores between morphed and baseline models were from the size (Table 3.6), while the same weighting factors were applied to the different CORA sub-scores (phase, size, correlation), so it would have been interesting to emphasize the size sub-score as higher variability was observed.

The analysis was performed up to $20 \mathrm{~ms}$ after the first head-vehicle contact to cover the main pedestrian response including head peak acceleration and vehicle-pedestrian interaction. Most injuries were assumed to have happened in this time period because the experimental setup was designed to avoid ground impact. Consequently, the biofidelity of THUMS PFEM during secondary impacts (ground impact) is beyond the scope of this study. 


\section{$\underline{\text { Injury prediction }}$}

In this study, both injury prediction methods captured injury results well with necropsy. Compared to the function-based method, the strain-based method predicts injury further to the more specific location, but it highly depends on injury thresholds, which were assumed to correlate with age and bone quality. In addition, the discrepancy on the phase shift between model strain and the PMHS showed the potential model improvement of capturing cortical bone strain for better use of the strain-based method.

Note that previous dummy tests (Matsui et al. 2005) and simulation studies (Han et al. 2012; Watanabe et al. 2012) showed very low thorax injury risk in sedan-pedestrian impact. Two reasons might have caused the serious thorax injuries observed in the current study and the experiments. First, in both the test and simulation, the elbows impacted the windshield area and didn’t provide any cushioning to protect the thorax, while elbow impacts were found to have great influence on head and thorax injury (Matsui et al. 2005; Paas et al. 2015; Chen et al. 2015, Chen et al. 2016). Second, the thorax impacted a stiff location on the vehicle between the cowl and windshield frame area and this increased the thorax injury risks.

Snedeker et al. (2005) found THUMS version 1.0 did well predicting PMHS pelvic fractures with $1 \%$ plastic strain threshold, while in this study morphed 2 captured fewer fractures than the PMHS tests using 1\% principle strain threshold, and underestimated the AIS 2+ risk of pelvic fractures as $11 \%$ using function-based prediction methods. The reasons could be the bone quality between the model and the PMHS, pelvis impact direction to the vehicle, or BLE height relative to the pelvis. Kerrigan et al. (2012) observed higher risk of cadaveric pelvic fractures in tests than in real world accidents, and attributed them to older subjects and pure lateral impact direction in experiments which was verified by simulation to be a significant factor influencing 
pelvic fracture risk (Chen et al. 2015). As the main injury resource for pelvis fracture in accidents (Chen et al. 2009), the BLE height relative to the pelvis is another significant factor (Simms et al. 2009). However, the high bone quality of PMHS 2 (BMD $0.975 \mathrm{~g} / \mathrm{cm} 2$ ) and good pelvis positioning (only $5 \mathrm{~mm}$ difference on pelvis height between morphed model and PMHS) should exclude these reasons. In test 2, the BLE was lower than the pelvis and no severe pelvis impact with the BLE was observed in the video, and the peak pelvis acceleration was under 40g (Figure 3.8) which is lower than the fracture tolerance of 130g for the US standard FMVSS 214 for pelvis in side impact, and also lower than 73g of reported peak acceleration of pelvic fracture in side impact tests on cadavers (Simms et al. 2009), so the reason for the serious PMHS pelvic fractures was unknown to the author.

The tibia plateau avulsion fractures observed in the test were not common in accidents (Kerrigan et al. 2012). It is suggested that the opening of the knee joint and a relatively low BMD (bone mineral density) in PMHS 1 (BMD $0.753 \mathrm{~g} / \mathrm{cm} 2$, Osteopenia) could be the cause in the experiments. With higher BMD, it was believed that the ligaments of PMHS 1 would tear instead of causing avulsion fractures. Though low BMD was not considered in the bone material of the human models in this study, consistent ligament injury locations in simulation and avulsion fractures on tibia plateau in test explained this mechanism.

Knee injuries, instead of long bone fractures, were observed in tests and simulations probably because the bumper height is very close to the knee height (Simms et al. 2009). Morphed 2 captured the avulsion fracture on the left ATFL ligament from fibula bone piece, and the injury mechanism was the left ankle inversion after the leg impacted with the bottom of the lower bumper. As a result of the leg impact in this area, morphed models predicted high strain on the left fibula close to the ATF. 
There is no consensus on strain threshold in previous studies. In this study, age-based 1.5\% threshold (Yasuki et al. 2010; Golman et al. 2014) was used for cortical bones of most body regions ( $1 \%$ for pelvis), and good injury predictions were observed for morphed models.

\section{Potential Model Improvement}

In this study, both morphed THUMS models exhibited less lateral bending on the spine and especially on the neck during the impact. By comparing with shoulder lateral impact tests with volunteers and cadavers, Paas et al. (2015) found the THUMS (v4.0) spine appeared to be stiffer than in the volunteers. Paas et al. (2015) evaluated the THUMS whole-body kinematics using recent 3-dimensional kinematics PMHS test data (Paas et al. 2015) and found that for the THUMS (v4.0) spine, and especially the neck, lateral bending was generally less pronounced than in the PMHSs. These studies supported the view that the THUMS spine might be stiffer than the cadavers. While the cadavers are known to lack muscle tone and thus are less stiff than a living human, the tissue failure could be a reason for softer spine in the tests since in both PMHS tests spine injuries were observed, but the influence of tissue failure on the pedestrian response has not been thoroughly investigated.

Knee ligament rupture is difficult to predict by strain, because previous literatures (Arnoux et al. 2008; Mo et al. 2014) showed great variability in knee ligament strain thresholds among studies from $7 \%$ to $60 \%$, and among ligament types due to different materials and injury mechanism since MCL failures are relevant to high bending values, while cruciate ligaments (especially ACL) mostly present high positive shearing values (Mo et al. 2014). But in this study both function-based (knee lateral bending angle) and strain-based methods overestimated the left knee ligament ruptures of morphed 2. Previous component evaluation on the lateral knee bending showed the knee moment angle responses of the baseline and morphed models were above the 
experimental data (Poulard et al. 2015). Therefore, both full-scale and component evaluation suggested potential improvement on the biofidelity of knee ligament.

Morphed models overestimated ligament ruptures in the ankle, and the reasons could be the biofidelity of ankle ligaments, the underestimated strain threshold, or an abnormal test result because video of test 1 showed great inversion of the left ankle but no injury was reported.

Future work should focus on those not well validated areas such as the spine, and soft tissues, including knee and ankle ligaments. Also, the influence of some intrinsic factors should be investigated, for example, the material properties varied by age and the relevance of bone quality on pedestrian response.

\subsubsection{Conclusion}

In this study, two morphed pedestrian finite element models (PFEM) were created to the anthropometric specifications of two obese PMHS used in a previous pedestrian impact study using a mid-size sedan. Kinematics and strain were compared to the experiments and injuries were predicted deterministically and using probalistic functions. The morphed THUMS models captured similar responses in terms of kinematics and injuries to those of the PMHSs, while the baseline models exhibited different response to PMHSs due to different anthropometries. Overall, THUMS v4.01 was deemed biofidelic on the extended data type reported and could be used as an advanced injury prediction tool in vehicle-pedestrian impact research and development, despite possible model improvements on the stiffness of the spine in lateral bending and further evaluation on the soft tissue. The findings of this study could be useful for improving model biofidelity for vehicle-pedestrian impact scenarios. 


\subsection{Evaluation of Morphed Models Against Small City Car}

The objective of this section is to evaluate the morphed THUMS PFEM biofidelity against the PMHS test data impacting with a small city car.

\subsubsection{Method}

Two male PMHS of different anthropometries (PMHS 3: 161 cm, 86.2 kg; PMHS 4: 182 $\mathrm{cm}, 46.3 \mathrm{~kg}$ ) were impacted laterally by a small city car buck traveling at $40 \mathrm{~km} / \mathrm{h}$. All subjects were in a walking posture with the left foot ahead and the right foot behind the body, and the arms bounded together at the wrists anterior to the thorax/abdomen. The postures were acquired from pre-test photographs and measurements of landmark heights and distances.

The details about vehicle profile, subjects' anthropometry, instrumentation, data processing, and injury report were published in previous literatures (Untaroiu et al. 2007; Subit et al. 2008; Poulard et al. 2016).

The same methods discussed in the chapter 3.2 were used in this section, on the model instrumentation, model setup, positioning, and CORA evaluation on segmental trajectory, velocity, acceleration, lower extremity strain, and two methods on injury prediction including probabilistic method and deterministic method.

\subsubsection{Results and Discussion}

The trajectories (Figure 3.11) and pedestrian impact sequences (Figure 3.12 - Figure 3.13) of both subjects indicated generally matched kinematical response of models against the PMHS data, especially on the upper body. Some differences were observed on the trajectory of subject 4 , especially on the lower extremity, while multiple fractures on the knee, tibia, and fibula were reported in the PMHS test 4 which could have been a major reason for this local difference on 
lower extremity trajectory. Compared with the pedestrian response against the mid-size sedan in the last section (Figure 3.3 and Figure 3.4), pedestrian impacted with the small city car (Figure 3.12 - Figure 3.13) always obtained less rotational kinematics, less sliding, and earlier head impact.
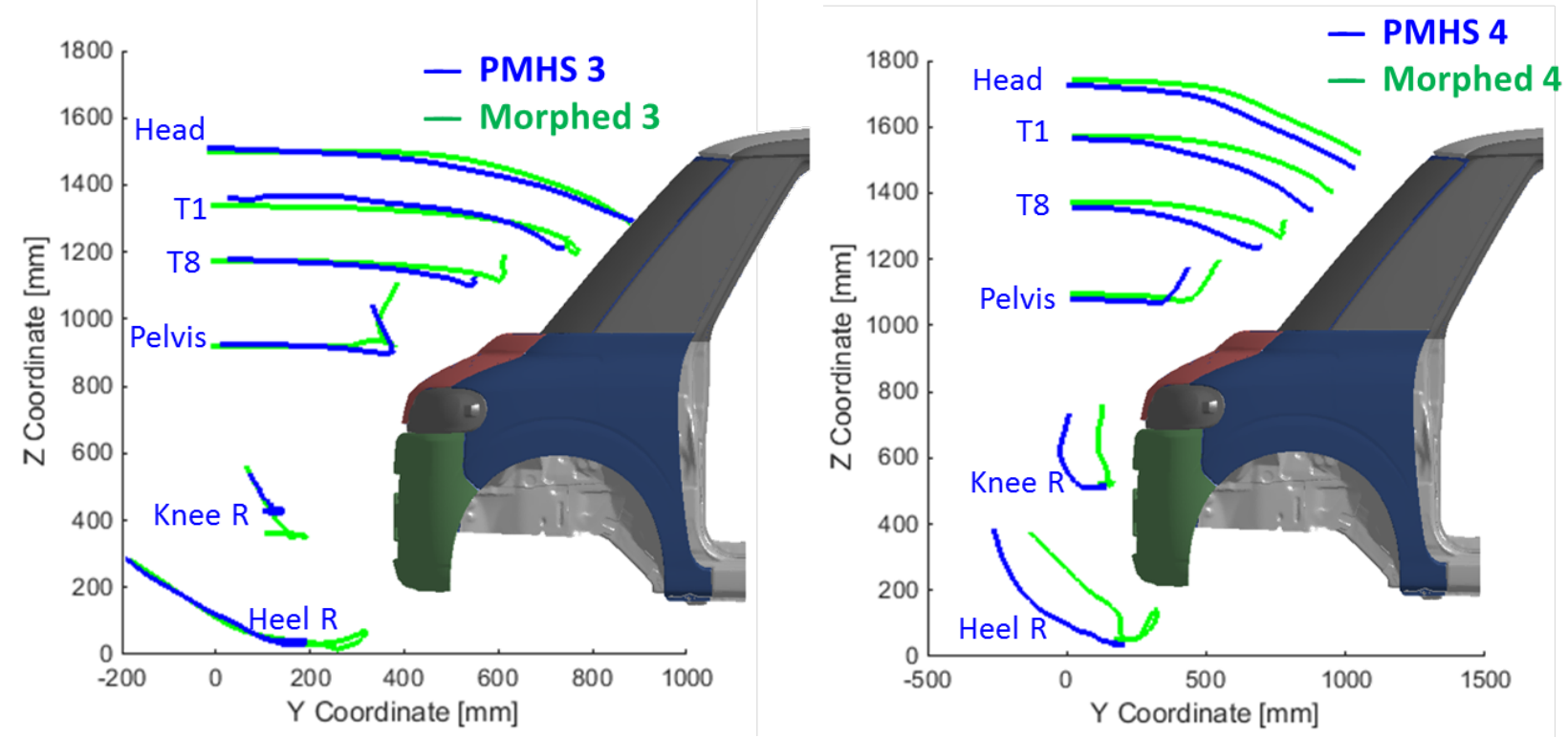

Figure 3.11 Trajectories in vehicle coordinate system (Left - subject 3, Right - subject 4).
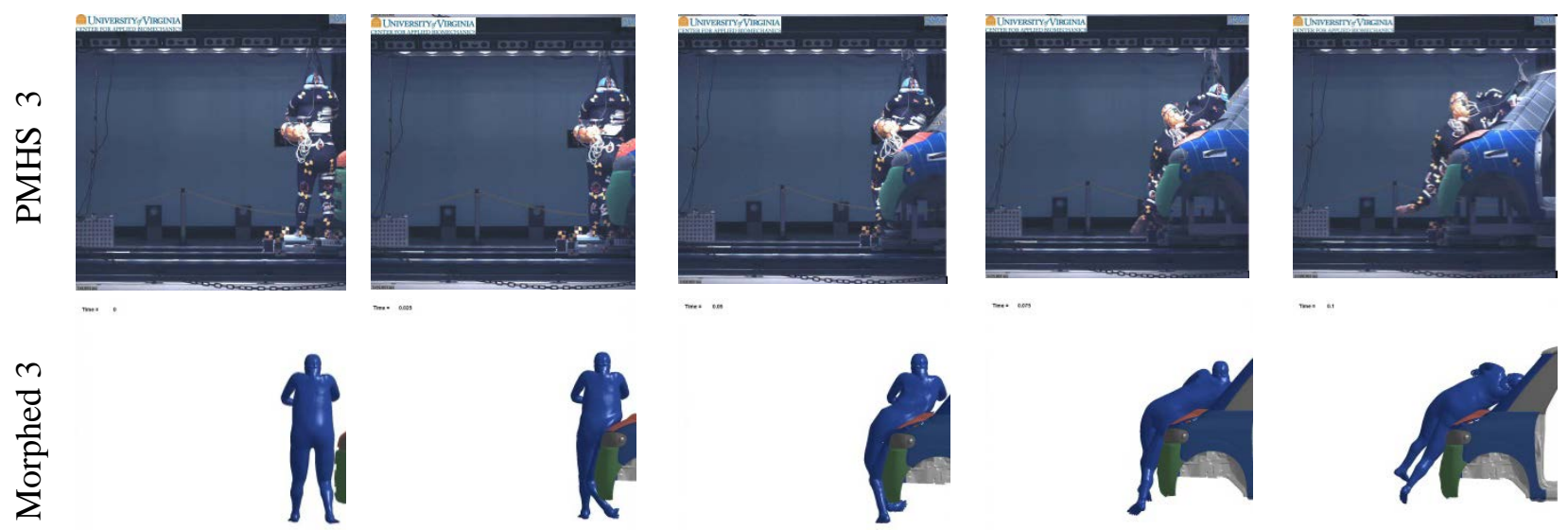

$0 \mathrm{~ms}$

$25 \mathrm{~ms}$

$50 \mathrm{~ms}$

$75 \mathrm{~ms}$

$100 \mathrm{~ms}$

Figure 3.12 Pedestrian impact sequences of PMHS and models (subject 3). 


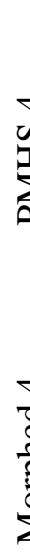
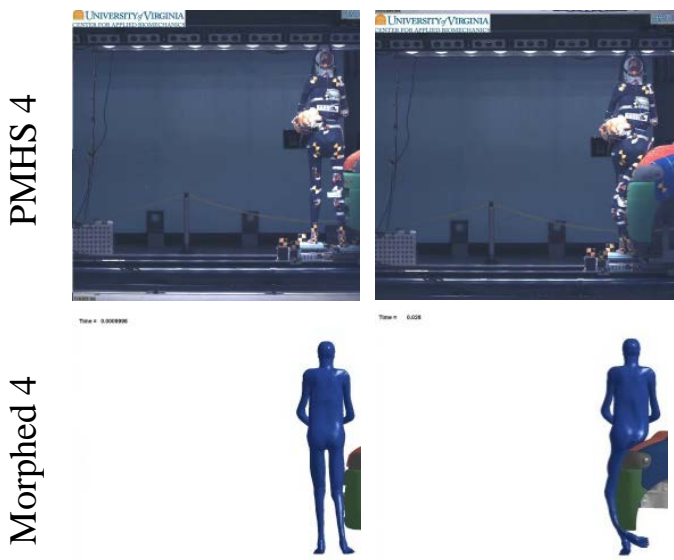

$0 \mathrm{~ms}$
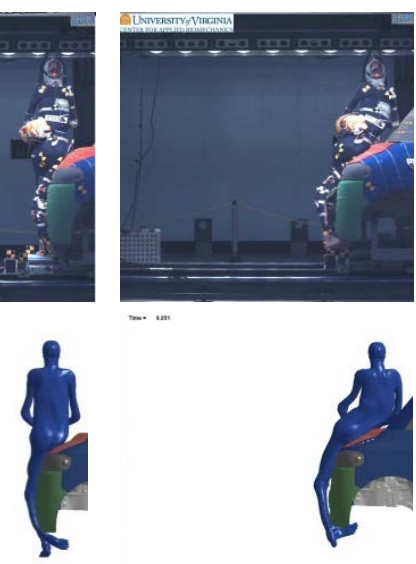

$25 \mathrm{~ms}$

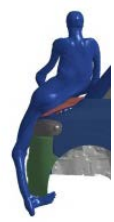

$50 \mathrm{~ms}$
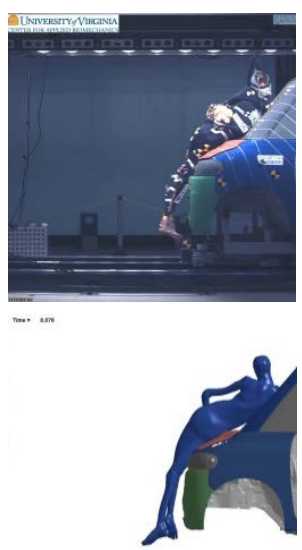

$75 \mathrm{~ms}$
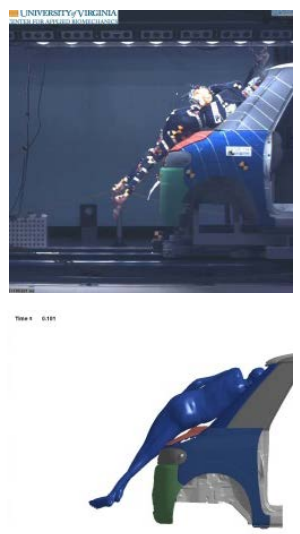

$100 \mathrm{~ms}$

Figure 3.13 Pedestrian impact sequences of PMHS and models (subject 4).

The morphed models obtained higher than 0.8 CORA scores with respect to the test results (see Table 3.9 - Table 3.10) on time history of resultant velocities, displacement, and acceleration of most body regions, while the strain time histories predicted by the morphed models were a little different to those observed in the experiments with regards to the magnitude and timing (Figure 3.18 - Figure 3.19), and lower CORA scores were observed on both subjects. This is also assumed to be due to the multiple fractures on the knee, tibia, and fibula reported in the PMHS test 4.

The time history curve of segmental trajectory, velocity, acceleration, and lower extremity strains (Figure 3.14 - Figure 3.19) were generally consistent with the CORA scores.

Table 3.9 Model evaluation results using CORA method (morphed 3, 4)

\begin{tabular}{ccccccccc}
\hline \multirow{2}{*}{$\begin{array}{c}\text { Physical } \\
\text { value }\end{array}$} & \multicolumn{9}{c}{ Morphed 3 } & $\begin{array}{c}\text { Morphed 4 } \\
\text { shift }\end{array}$ & Size & $\begin{array}{c}\text { Cross } \\
\text { correlation }\end{array}$ & $\begin{array}{c}\text { Total } \\
\text { rating }\end{array}$ & $\begin{array}{c}\text { Phase } \\
\text { shift }\end{array}$ & Size & $\begin{array}{c}\text { Cross } \\
\text { correlation }\end{array}$ & $\begin{array}{c}\text { Total } \\
\text { rating }\end{array}$ \\
\hline Displacement & 0.963 & 0.657 & 0.979 & 0.866 & 0.890 & 0.683 & 0.945 & 0.839 \\
Velocity & 1.000 & 0.771 & 0.993 & 0.921 & 1.000 & 0.850 & 0.992 & 0.947 \\
Acceleration & 0.832 & 0.447 & 0.930 & 0.737 & 0.936 & 0.631 & 0.920 & 0.829 \\
\hline
\end{tabular}




\begin{tabular}{ccccccccc}
\hline Strain & 0.660 & 0.406 & 0.805 & 0.624 & 0.576 & 0.316 & 0.872 & 0.588 \\
\hline
\end{tabular}

Table 3.10 CORA ratings (Subject 3, 4)

\begin{tabular}{|c|c|c|c|c|c|c|c|c|c|}
\hline \multirow[b]{2}{*}{$\begin{array}{l}\text { Physical } \\
\text { value }\end{array}$} & \multirow[b]{2}{*}{ Location } & \multicolumn{4}{|c|}{ Morphed 3} & \multicolumn{4}{|c|}{ Morphed 4} \\
\hline & & $\begin{array}{l}\text { Phase } \\
\text { shift }\end{array}$ & Size & $\begin{array}{c}\text { Cross } \\
\text { correlation }\end{array}$ & $\begin{array}{l}\text { Total } \\
\text { rating }\end{array}$ & $\begin{array}{l}\text { Phase } \\
\text { shift }\end{array}$ & Size & $\begin{array}{c}\text { Cross } \\
\text { correlation }\end{array}$ & $\begin{array}{l}\text { Total } \\
\text { rating }\end{array}$ \\
\hline \multirow{12}{*}{ 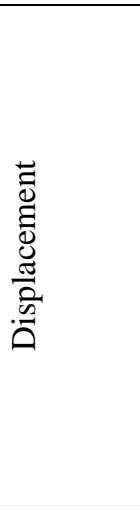 } & Head Y & 1.000 & 0.903 & 0.997 & 0.967 & 1.000 & 0.889 & 0.998 & 0.962 \\
\hline & Head Z & 1.000 & 0.957 & 0.999 & 0.985 & 1.000 & 0.876 & 0.999 & 0.958 \\
\hline & $\mathrm{T} 1 \mathrm{Y}$ & 1.000 & 0.776 & 0.998 & 0.925 & 1.000 & 0.824 & 0.999 & 0.941 \\
\hline & $\mathrm{T} 1 \mathrm{Z}$ & 1.000 & 0.791 & 0.993 & 0.928 & 1.000 & 0.524 & 0.998 & 0.841 \\
\hline & T8 Y & 1.000 & 0.670 & 0.998 & 0.889 & 1.000 & 0.732 & 0.999 & 0.910 \\
\hline & T8 Z & 0.633 & 0.461 & 0.982 & 0.692 & 0.684 & 0.454 & 0.985 & 0.708 \\
\hline & Pelvis Y & 1.000 & 0.890 & 0.999 & 0.963 & 1.000 & 0.643 & 0.998 & 0.880 \\
\hline & Pelvis Z & 1.000 & 0.332 & 0.959 & 0.764 & 1.000 & 0.714 & 0.999 & 0.904 \\
\hline & Knee R Y & 0.920 & 0.198 & 0.943 & 0.687 & 0.000 & 0.226 & 0.505 & 0.244 \\
\hline & Knee R Z & 1.000 & 0.333 & 0.993 & 0.775 & 1.000 & 0.949 & 0.998 & 0.982 \\
\hline & Heel R Y & 1.000 & 0.736 & 0.907 & 0.881 & 1.000 & 0.471 & 0.869 & 0.780 \\
\hline & Heel R Z & 1.000 & 0.836 & 0.978 & 0.938 & 1.000 & 0.893 & 0.988 & 0.960 \\
\hline \multirow{6}{*}{$\begin{array}{l}\stackrel{3}{0} \\
\frac{0}{0} \\
\end{array}$} & Head & 1.000 & 0.643 & 0.990 & 0.878 & 1.000 & 0.666 & 0.991 & 0.886 \\
\hline & $\mathrm{T} 1$ & 1.000 & 0.681 & 0.992 & 0.891 & 1.000 & 0.987 & 0.991 & 0.993 \\
\hline & $\mathrm{T} 8$ & 1.000 & 0.871 & 0.994 & 0.955 & 1.000 & 0.696 & 0.991 & 0.896 \\
\hline & Pelvis & 1.000 & 0.785 & 0.993 & 0.926 & 1.000 & 0.936 & 0.996 & 0.977 \\
\hline & Knee R & 1.000 & 0.811 & 0.993 & 0.935 & 1.000 & 0.933 & 0.993 & 0.975 \\
\hline & Heel R & 1.000 & 0.836 & 0.995 & 0.944 & 1.000 & 0.879 & 0.990 & 0.956 \\
\hline \multirow{9}{*}{ 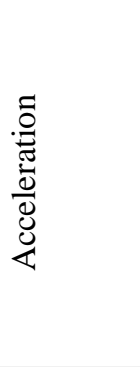 } & Head & 0.670 & 0.778 & 0.967 & 0.805 & 1.000 & 0.688 & 0.937 & 0.875 \\
\hline & $\mathrm{T} 1$ & 0.622 & 0.283 & 0.951 & 0.619 & 0.820 & 0.422 & 0.903 & 0.715 \\
\hline & Pelvis & 0.859 & 0.747 & 0.909 & 0.838 & 1.000 & 0.723 & 0.903 & 0.875 \\
\hline & Femur L & 0.820 & 0.279 & 0.953 & 0.684 & 0.868 & 0.466 & 0.969 & 0.768 \\
\hline & Femur R & 0.921 & 0.233 & 0.921 & 0.692 & 1.000 & 0.392 & 0.918 & 0.770 \\
\hline & Tibia L & 0.859 & 0.379 & 0.949 & 0.729 & 0.943 & 0.577 & 0.921 & 0.813 \\
\hline & Tibia R & 0.886 & 0.462 & 0.906 & 0.751 & 0.987 & 0.524 & 0.927 & 0.813 \\
\hline & Ankle L & 0.965 & 0.382 & 0.933 & 0.760 & 0.917 & 0.950 & 0.918 & 0.928 \\
\hline & Ankle R & 0.886 & 0.482 & 0.884 & 0.751 & 0.886 & 0.940 & 0.880 & 0.902 \\
\hline \multirow{12}{*}{ 䨠 } & LF 1 & 0.929 & 0.202 & 0.921 & 0.684 & 0.969 & 0.064 & 0.835 & 0.623 \\
\hline & LF 2 & 1.000 & 0.843 & 0.745 & 0.862 & & & & \\
\hline & LF 3 & 0.914 & 0.642 & 0.776 & 0.777 & 0.708 & 0.175 & 0.927 & 0.603 \\
\hline & LF 4 & 0.951 & 0.740 & 0.868 & 0.853 & 0.000 & 0.434 & 0.688 & 0.374 \\
\hline & RF 5 & 0.600 & 0.033 & 0.856 & 0.496 & 0.798 & 0.654 & 0.927 & 0.793 \\
\hline & RF 6 & 0.565 & 0.355 & 0.905 & 0.608 & 0.769 & 0.405 & 0.917 & 0.697 \\
\hline & RF 7 & 0.000 & 0.003 & 0.501 & 0.168 & 0.843 & 0.724 & 0.776 & 0.781 \\
\hline & RF 8 & 0.580 & 0.543 & 0.921 & 0.681 & 0.518 & 0.549 & 0.956 & 0.675 \\
\hline & RT 9 & 0.863 & 0.680 & 0.929 & 0.824 & 0.353 & 0.069 & 0.907 & 0.443 \\
\hline & RT 10 & 0.863 & 0.391 & 0.921 & 0.725 & 0.310 & 0.231 & 0.909 & 0.483 \\
\hline & RT 11 & & & & & 0.749 & 0.020 & 0.913 & 0.561 \\
\hline & RT 12 & 0.000 & 0.032 & 0.518 & 0.183 & 0.320 & 0.146 & 0.833 & 0.433 \\
\hline
\end{tabular}



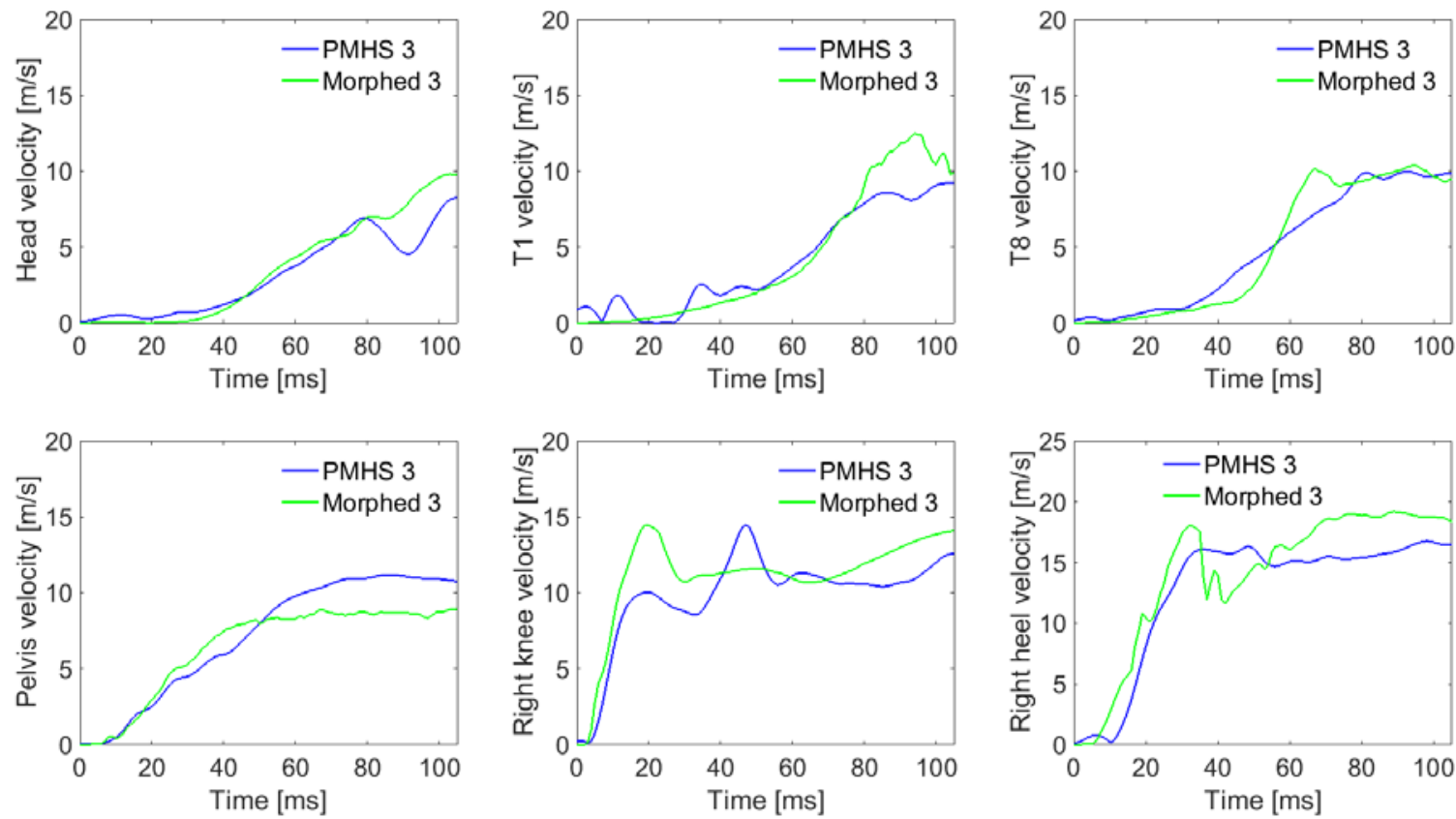

Figure 3.14 Time history of resultant velocity on each body region (subject 3)
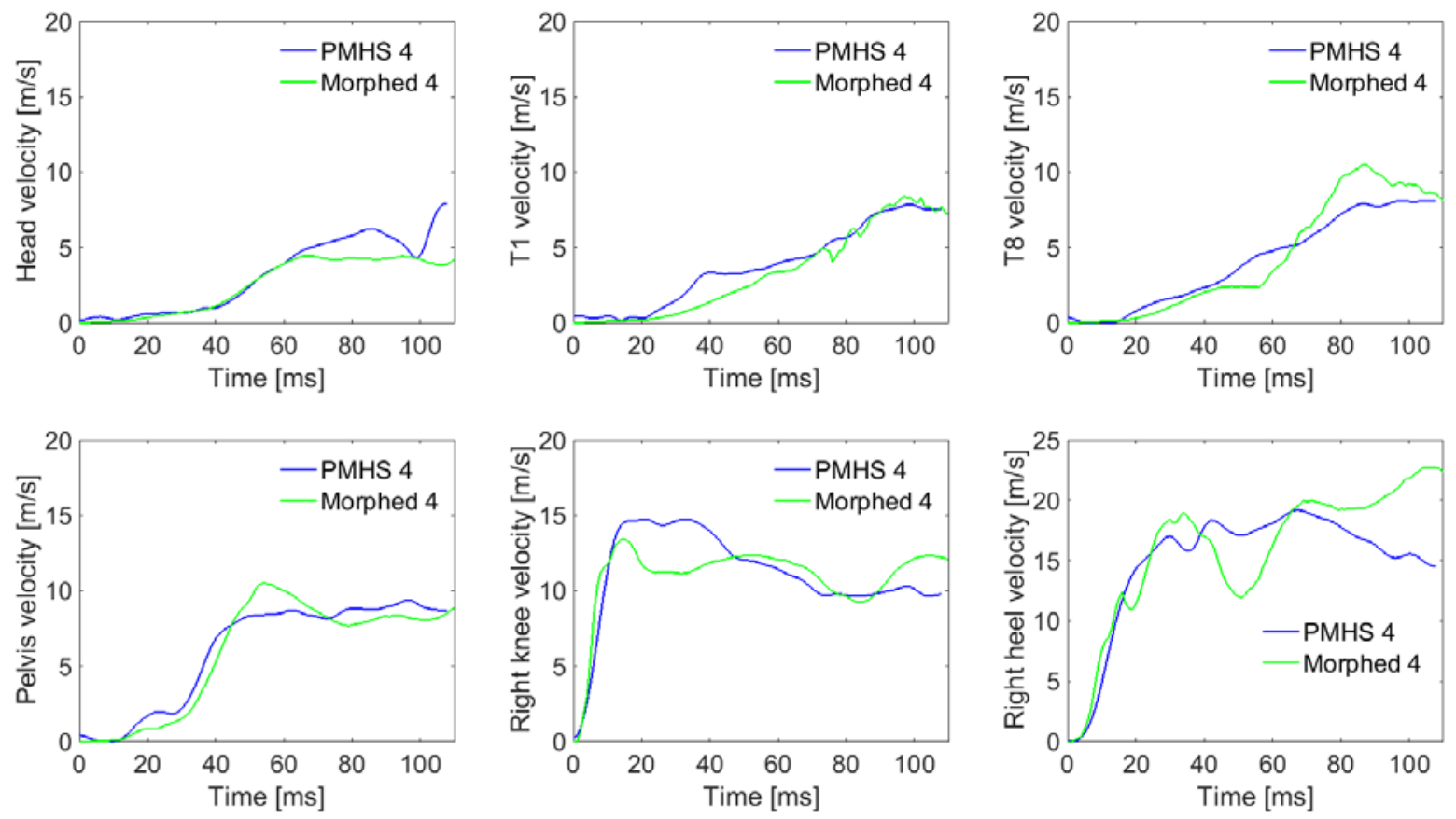

Figure 3.15 Time history of resultant velocity on each body region (subject 4) 

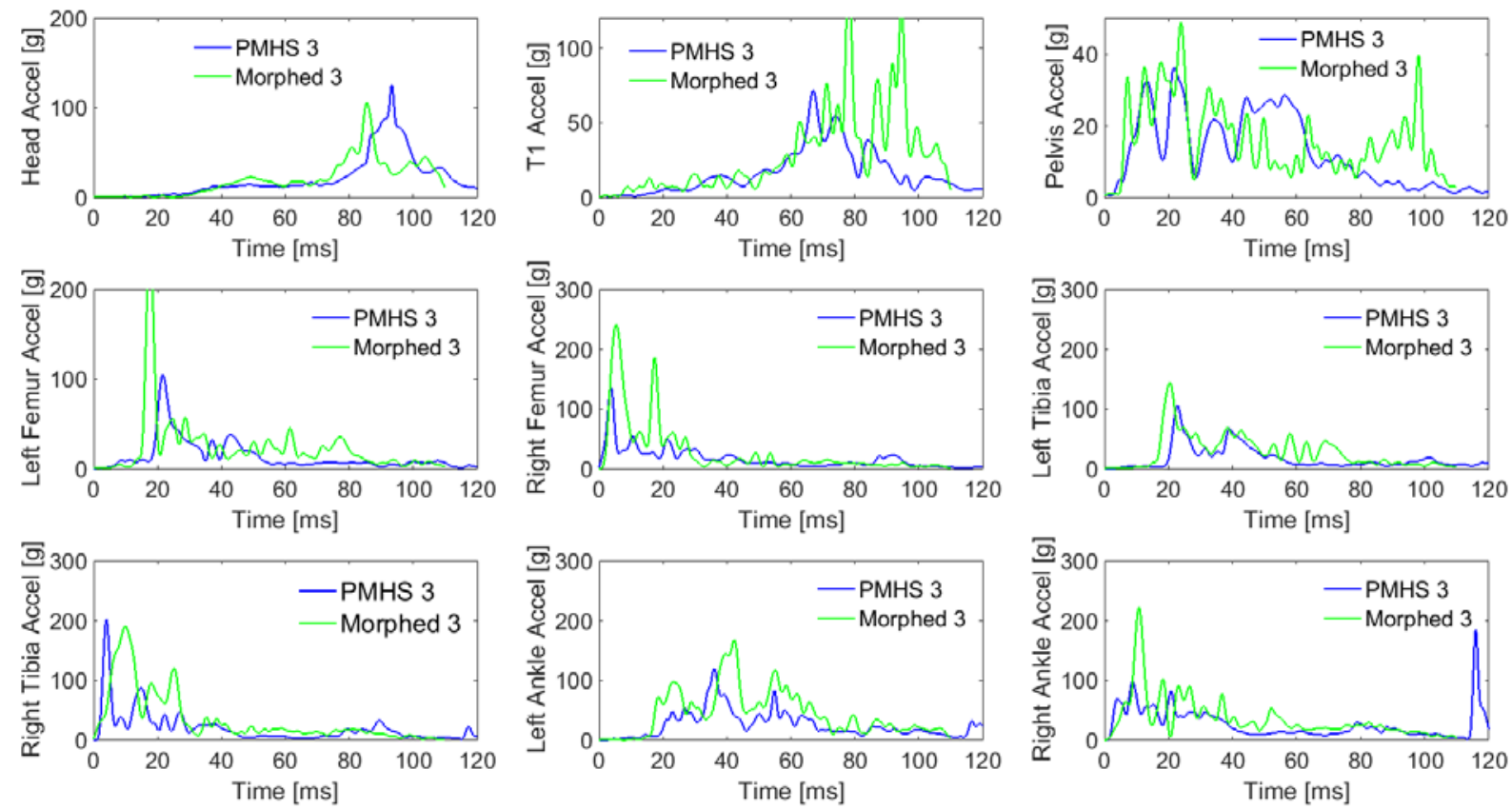

Figure 3.16 Time history of resultant acceleration on each body region (subject 3)
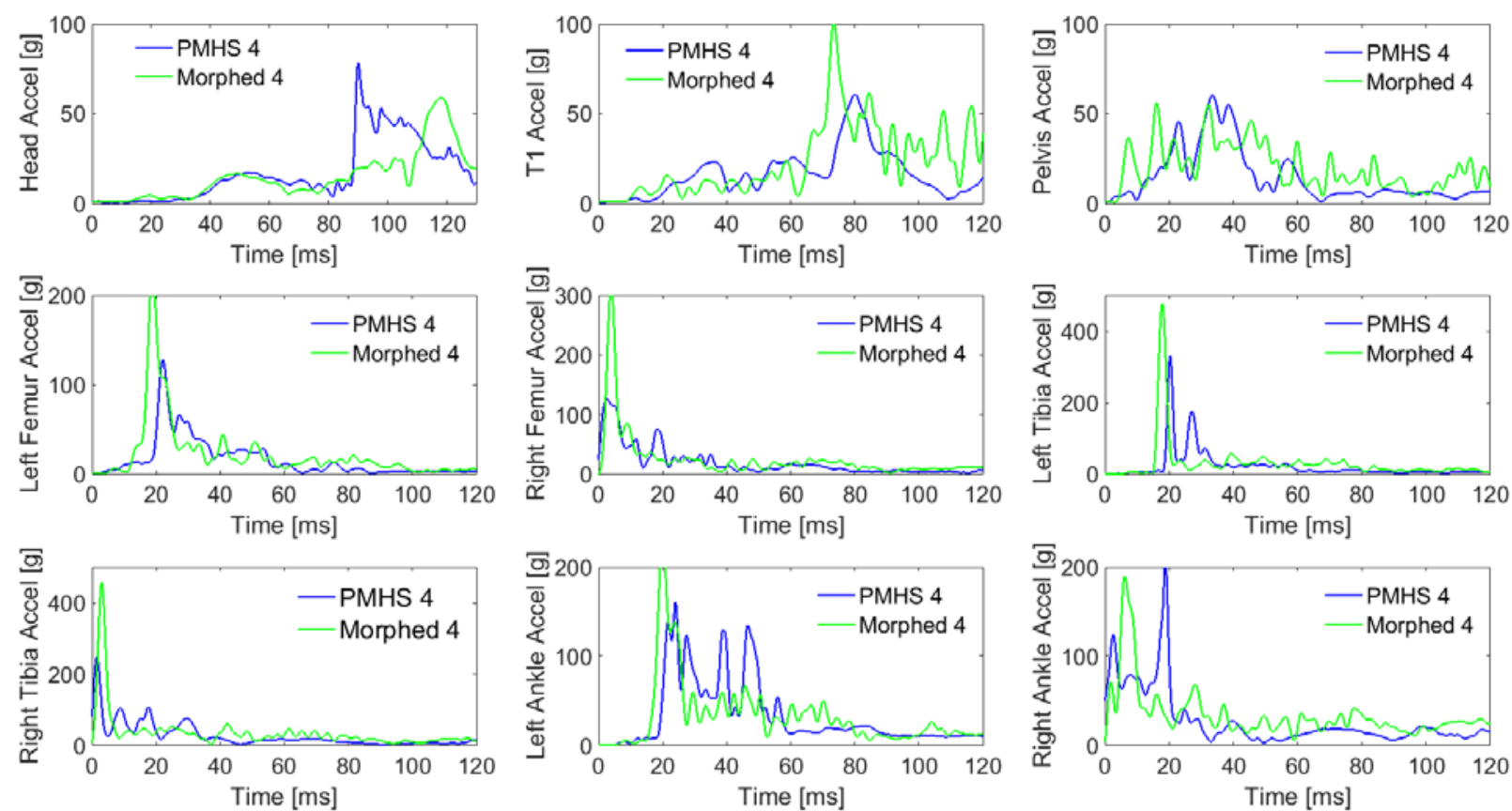

Figure 3.17 Time history of resultant acceleration on each body region (subject 4) 

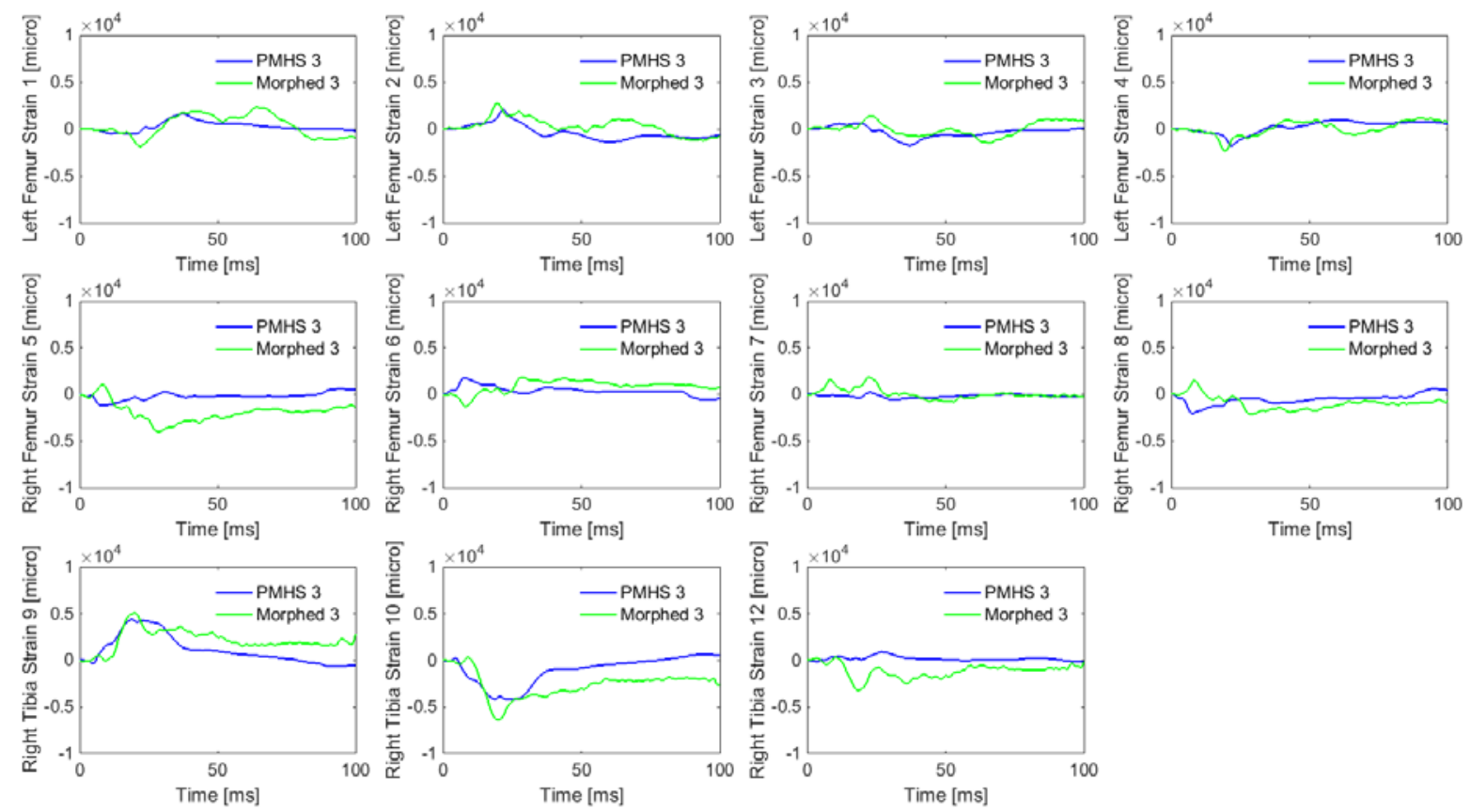

Figure 3.18 Time history of lower extremity strain of subject 3
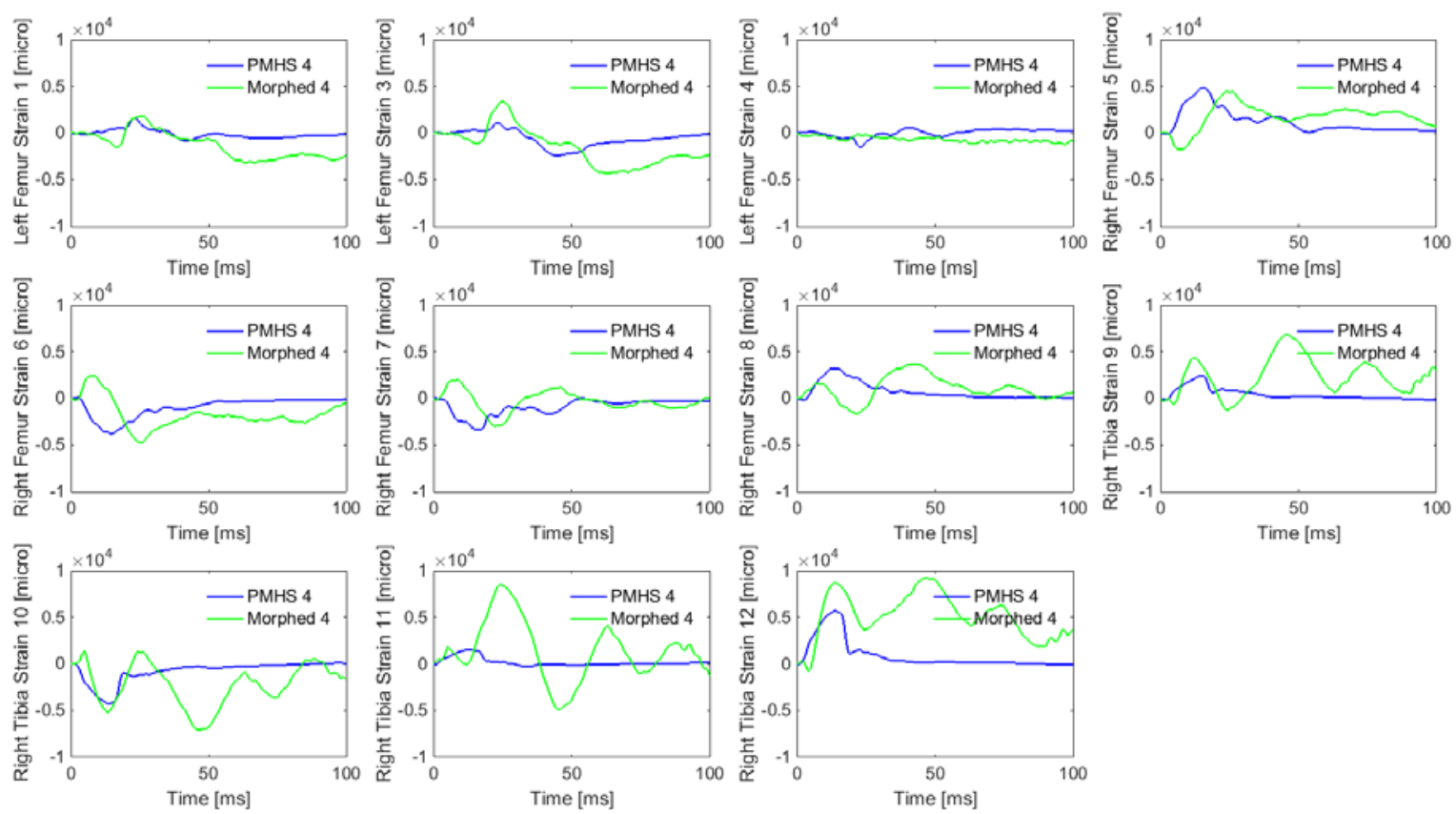

Figure 3.19 Time history of lower extremity strain of subject 4 
Morphed models generally captured injury risks of the body regions of both subjects (Table 3.11 - Table 3.12, and Figure 3.20- Figure 3.21).

For subject 3, using the probabilistic method (PM), morphed 3 predicted consistent injury risk on head and lower extremity, while slightly overestimated the pelvic injury risk and underestimated the rib fractures. Using the deterministic method (DM), morphed 3 captured neck ligament rupture, and ligament ruptures on right knee and left ankle, but predicted less rib fractures as in the experiment, and overestimated the shoulder fracture, and ligament ruptures on left knee and right ankle.

For subject 4, using the probabilistic method (PM), morphed 4 predicted consistent injury risk on head, thorax, and lower extremity, while underestimated the pelvic injury risk. Using the deterministic method (DM), morphed 4 captured left knee and right leg injury risk, but predicted less rib fractures and pelvic fractures as in the experiment, and overestimated the right femur and left leg fracture, and ankle ligament ruptures on both sides.

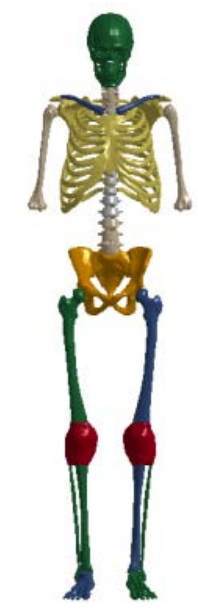

Morphed 3 (PM)

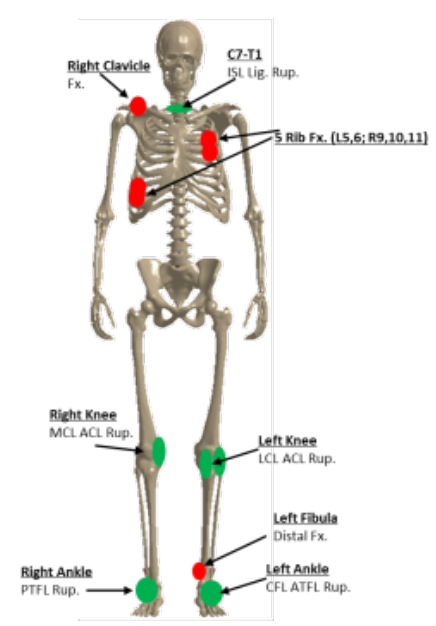

Morphed 3 (DM)

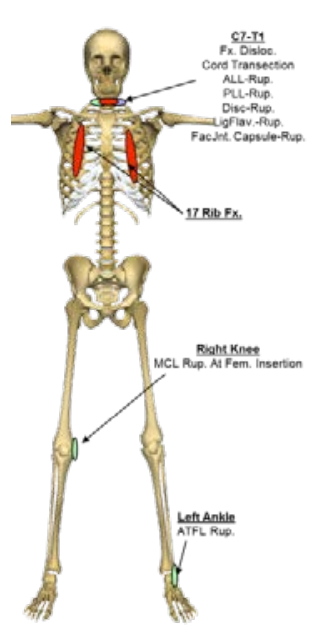

PMHS 3

Figure 3.20 Comparison of injury prediction of subject 3 for morphed model and PMHS injury from Subit et al. 2008 


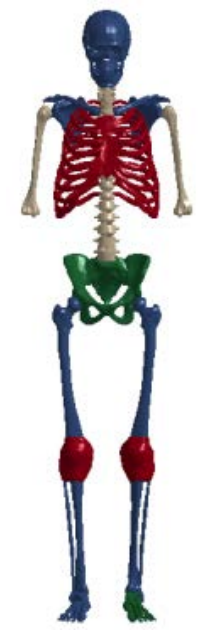

Morphed 4 (PM)

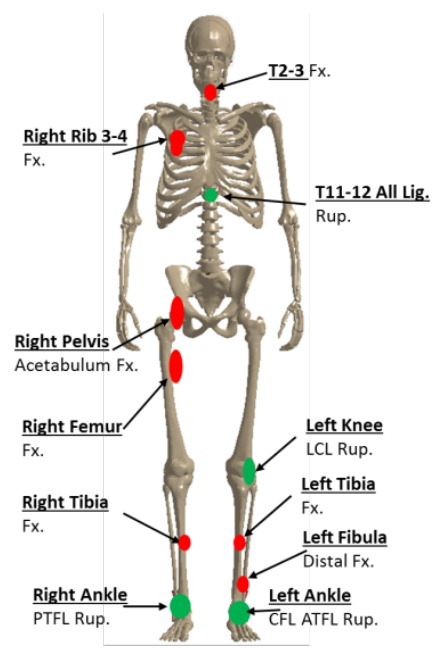

Morphed 4 (DM)

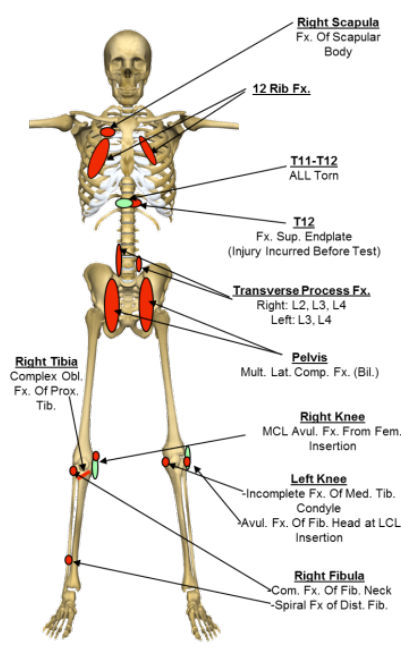

PMHS 4

Figure 3.21 Comparison of injury prediction of subject 4 for morphed model and PMHS injury from Subit et al. 2008

Table 3.11 Summary of predicted injury for PMHS 3 and Morphed 3 using probabilistic (PM) and deterministic method (DM) (Model prediction inconsistent with PMHS are bolded and underlined).

\begin{tabular}{|c|c|c|c|c|}
\hline \multirow{2}{*}{ Body region } & \multirow{2}{*}{ Predicted injury } & \multirow{2}{*}{ PMHS 3} & \multicolumn{2}{|c|}{ Morphed 3} \\
\hline & & & $\mathbf{P M}$ & $\mathbf{D M}$ \\
\hline Head & Skull Fx. & No & $10.6 \%(\mathrm{AIS} 2+)$ & No \\
\hline Neck (soft) & Ligament Rup. & C7-T1 all lig. Rup. & Not eva. & All Lig. Rup. \\
\hline Neck (bone) & Vertebrae Fx. & No & Not eva. & No \\
\hline Thorax R & Rib Fx. & Rib 2-11 Fx & $32.8 \%($ AIS3+) & Rib 8- $10 \mathrm{Fx}$ \\
\hline Thorax L & Rib Fx. & Rib 4-7 Fx. & $16.1 \%$ (AIS3+) & Rib 5, 6 Fx. \\
\hline Thorax M & Sternum Fx. & No & Not eva. & No \\
\hline Shoulder R & Clav/scap Fx. & No & $0 \%(\mathrm{AIS} 2+)$ & Clavicle \\
\hline Shoulder L & Clav/scap fx. & No & $0 \%(\mathrm{AIS} 2+)$ & No \\
\hline Spine (soft) & Ligament Rup. & No & Not eva. & All Lig. Rup. \\
\hline Spine (bone) & Vertebrae Fx. & No & Not eva. & No \\
\hline Pelvis R & Pelvis Fx. & No & \multirow{2}{*}{$51.8 \%($ AIS2+) } & No \\
\hline Pelvis L & Pelvis Fx. & No & & No \\
\hline R Thigh & Femur Fx. & No & $6.0 \%(\mathrm{AIS} 3+)$ & No \\
\hline L Thigh & Femur Fx. & No & $0.6 \%(\mathrm{AIS3}+)$ & No \\
\hline R Leg & Tibia/fibula Fx. & No & $8.3 \%(\mathrm{AIS} 2+)$ & No \\
\hline L Leg & Tibia/fibula Fx. & No & $0.2 \%(\mathrm{AIS} 2+)$ & Fib. Fx. \\
\hline R Knee (soft) & Ligament Rup. & MCL Rup. & $100 \%(\mathrm{AIS} 2+)$ & $\overline{\text { MCL Rup. }}$ \\
\hline R Knee (bone) & Tibia plat. Fx. & No & $0.4 \%(\mathrm{AIS} 2+)$ & No \\
\hline L Knee (soft) & Ligament Rup. & No & $100 \%$ (AIS2+) & LCL ACL Rup. \\
\hline L Knee (bone) & Tibia plat. Fx. & No & $0.6 \%(\mathrm{AIS} 2+)$ & No \\
\hline R Ankle (soft) & Ligament Rup. & No & \multirow{2}{*}{$0.0 \%(\mathrm{AIS} 2+)$} & PTFL Rup. \\
\hline R Ankle(bone) & Calcaneus/Talus Fx. & No & & No \\
\hline L Ankle (soft) & Ligament Rup. & ATFL Rup. & \multirow{2}{*}{$9.8 \%(\mathrm{AIS} 2+)$} & CFL ATFL Rup. \\
\hline L Ankle (bone) & Calcaneus/Talus Fx. & No & & No \\
\hline
\end{tabular}


Table 3.12 Summary of predicted injury for PMHS 4 and Morphed 4 using probabilistic (PM) and deterministic method (DM) (Model prediction inconsistent with PMHS are bolded and underlined).

\begin{tabular}{ccccc}
\hline \multirow{2}{*}{ Body region } & \multirow{2}{*}{ Predicted injury } & PMHS 4 & PM & Morphed 4 \\
\cline { 3 - 5 } Head & Skull Fx. & No & $3.2 \%$ (AIS2+) & No \\
Neck (soft) & Ligament Rup. & No & Not eva. & No \\
Neck (bone) & Vertebrae Fx. & No & Not eva. & C1 Fx. \\
Thorax R & Rib Fx. & Rib 1,3,4,6,12 Fx. & $92.0 \%$ (AIS3+) & Rib 3, 4 Fx \\
Thorax L & Rib Fx. & Rib 2-6 Fx. & $1.1 \%$ (AIS3+) & No \\
Thorax M & Sternum Fx. & No & Not eva. & No \\
Shoulder R & Clav/scap Fx. & Scap. Fx. & $0.0 \%$ (AIS2+) & Scap Fx. \\
Shoulder L & Clav/scap fx. & No & $0.0 \%$ (AIS2+) & No \\
Spine (soft) & Ligament Rup. & T11-12 All Rup. & Not eva. & T11-12 All Lig. Rup. \\
Spine (bone) & Vertebrae Fx. & T12 L2-4 Fx. & Not eva. & T2-3 Fx. \\
Pelvis R & Pelvis Fx. & Mult. Fx. & $\mathbf{7 . 7 \% ( A I S 2 + )}$ & Acetabulum Fx. \\
Pelvis L & Pelvis Fx. & Mult. Fx. & \multirow{2}{*}{ No } \\
R Thigh & Femur Fx. & No & $0.1 \%$ (AIS3+) & Femur Fx. \\
L Thigh & Femur Fx. & No & $0.0 \%$ (AIS3+) & No \\
R Leg & Tibia/fibula Fx. & Fib. Fx. & $0.1 \%$ (AIS2+) & Tib. Fx. \\
L Leg & Tibia/fibula Fx. & No & $0.0 \%$ (AIS2+) & $\underline{\text { Tib. Fib. Fx. }}$ \\
R Knee (soft) & Ligament Rup. & MCL Rup. & $100 \%$ (AIS2+) & No \\
R Knee (bone) & Tibia plat. Fx. & No & $8.3 \%$ (AIS2+) & No \\
L Knee (soft) & Ligament Rup. & LCL Rup. & $100 \%$ (AIS2+) & LCL Rup. \\
L Knee (bone) & Tibia plat. Fx. & No & $4.3 \%$ (AIS2+) & No \\
R Ankle (soft) & Ligament Rup. & No & $5.1 \%$ (AIS2+) & $\underline{\text { PTFL Rup. }}$ \\
R Ankle(bone) & Calcaneus/Talus Fx. & No & No \\
L Ankle (soft) & Ligament Rup. & No & $20.4 \%$ (AIS2+) & $\underline{\text { CFL ATFL Rup. }}$ \\
L Ankle (bone) & Calcaneus/Talus Fx. & No & No \\
\hline
\end{tabular}

\subsubsection{Conclusion}

In this study, two morphed pedestrian finite element models (PFEM) were created to the anthropometric specifications of one obese and one skinny PMHS used in a previous pedestrian impact study using a small city car. Kinematics and strain were compared to the experiments and injuries were predicted deterministically and using probabilistic functions. The morphed THUMS models captured similar responses in terms of kinematics and injuries to those of the PMHSs, while the local differences on lower extremity response of the skinny subject were assumed to be due to the massive local tissue failure. Overall THUMS v4.01 was deemed 
biofidelic on the extended data type reported despite possible model improvements and further evaluation needed to more accurately capture the pedestrian injury risks.

\subsection{Effect of Enabling Tissue Failure}

The objective of this study is to computationally investigate the influence of pedestrian bone mineral density and enabling tissue failure on its kinematics and injury risk. The results showed that the pedestrian kinematics is insensitive to bone quality, while enabling tissue failure only has local effect on kinematics of severe injured area (lower extremities), but has little effect on the pedestrian upper body kinematics. Also, the pedestrian whole body injury risk didn't show significant sensitivity on the bone quality or enabling the tissue failure.

\subsubsection{Background}

Previous PFEM model evaluation using personalized geometry models (Chen et al. 2017) considered the effect of specific anthropometry of different subjects, but there are still some factors that have not been considered in the model evaluation study, including the bone quality and the potential tissue failure.

The subjects used in the PMHS tests are generally very old (Paas et al. 2016; Subit et al. 2008; Kerrigan et al. 2005), and these aged subjects might have lower bone density, which might change their bone material properties and injury threshold (Watanabe et al. 2012). Also, many injuries were observed in the tests (Subit et al. 2008), while previous studies didn't enable these tissue failures in the simulation (Chen et al. 2017; Paas et al. 2016), so the effect of the tissue failure on the following pedestrian response needs to be verified.

A previous study (Dunmore et al. 2005) on the effect of bone quality and fracture on pedestrian response only focused on knee injury risk, and used simplified MADYMO human and 
vehicle models. Recently, the pedestrian finite element model (PFEM) offers some promising advantages as advanced injury prediction tools in vehicle-pedestrian impacts: detailed anatomical information, improved material modes and injury prediction at the tissue level. One of the existing PFEMs is the Total Human Model for Safety (THUMS) pedestrian model (TMC 2011), which has been comprehensively validated at the component level (Maeno et al. 2001; Iwamoto et al. 2002; TMC 2011; Poulard et al. 2015) and full-scale level (Watanabe et al. 2012; Paas et al. 2015; Poulard et al. 2016; Chen et al. 2017), and widely used to investigate the biomechanics in vehicle-pedestrian impact (Snedeker et al. 2003; Watanabe et al. 2012; Han et al. 2012; Chen et al. 2015). This model provides a more advanced tool for further investigation on the effect of pedestrian bone mineral density and enabling tissue failure on its kinematics and injury risk.

The objective of this study is to computationally investigate the influence of pedestrian bone mineral density and enabling tissue failure on its kinematics and injury risk.

\subsubsection{Method}

\section{Baseline models}

Two previously published simulation results (Chen et al. 2017) are used as the baseline responses. Those two simulations were to model two male PMHS with different anthropometries, age, and bone density (subject 1: $154 \mathrm{~cm}, 72.6 \mathrm{~kg}, 62$ years old, osteopenia; subject 2: $183 \mathrm{~cm}$, $114 \mathrm{~kg}$, 62 years old, normal) impacted laterally by a mid-size sedan buck traveling at $40 \mathrm{~km} / \mathrm{h}$. Both subjects were in a walking posture with the left foot ahead and the right foot behind the body, and the arms bounded together at the wrists anterior to the thorax/abdomen. The postures were acquired from pre-test photographs and measurements of landmark heights and distances. 
The details about vehicle profile and vehicle model validation, subjects’ anthropometry, instrumentation, data processing, and injury report were published in previous literatures (Untaroiu et al. 2007; Subit et al. 2008; Watanabe et al. 2012; Poulard et al 2016; Chen et al. 2017).

The baseline models used the material properties of original THUMS model, and didn’t enable the tissue failure during the vehicle-pedestrian impact.

\section{$\underline{\text { Simulation matrix }}$}

Six additional simulations were performed to investigate the effect of bone quality and tissue failure. The effect of bone density was studied by varying young's modulus of the cortical bone. Since the original THUMS model was reported to model a young male (Wanatabe et al. 2012), it was assumed that the bone quality will represent the same population. Consequently, to account for a decrease in bone quality as reported in vulnerable populations, the young's modulus was proportionally reduced by $10 \%$ on all cortical bones of the model.

The effect of tissue failure was studied by enabling the element deletion of cortical bones, when beyond the defined threshold of $0.88 \%$ plastic strain on cortical bones (Untaroiu et al. 2005; Nie et al. 2016). Three levels of enabling tissue failure were performed: No enabling (baseline models); Enabling of lower extremity failures, including knee ligament rupture, femur fractures, and tibia/fibula fractures; Enabling of lower extremity failures, pelvic fracture, and rib fractures. The corresponding simulation matrix was listed in Table 3.13. 
Table 3.13 Simulation matrix

\begin{tabular}{cccc}
\hline Case No. & Subject & Tissue failure & Bone quality (young's modulus) \\
\hline 1 & 1 (shorter) & No enabling & baseline \\
2 & 1 & No enabling & $10 \%$ lower on cortical bone \\
3 & 1 & Lower extremities & baseline \\
4 & 1 & Lower extremities + pelvis + rib & baseline \\
5 & 2 (taller) & No enabling & baseline \\
6 & 2 & No enabling & $10 \%$ lower on cortical bone \\
7 & 2 & Lower extremities & baseline \\
8 & 2 & Lower extremities + pelvis + rib & baseline \\
\hline
\end{tabular}

\section{$\underline{\text { Response comparison }}$}

The whole-body kinematics was compared with the baseline models, including segmental trajectory, velocity, and acceleration. The trajectories of the head, spine, pelvis, right knee (KneeR), and right heel (HeelR) were assessed in the vehicle coordinate system (VCS) (Figure 3.22). Triaxial accelerations were measured by derivation of the velocity measured on a node attached to the center of the bony location (head, T1/T2, T8/T9, L5, pelvis, femur, and ankle). Simulation data was computed at $1000 \mathrm{~Hz}$ for displacement and velocity and $10 \mathrm{KHz}$ for acceleration and digitally filtered with a CFC180 SAE channel class filter as it was in the experiments.
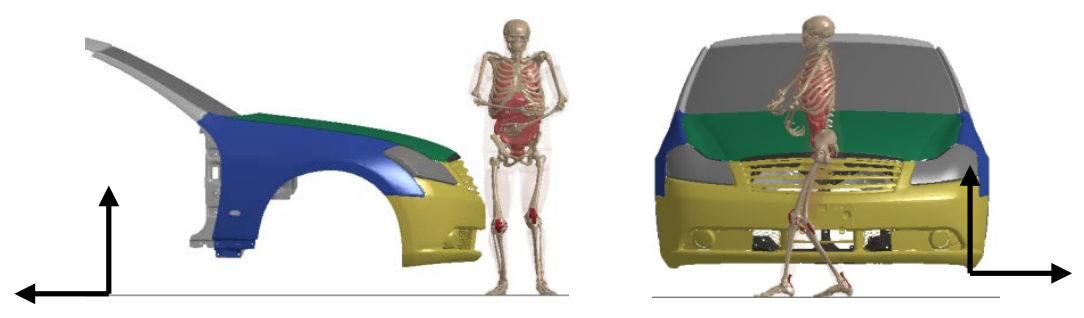

Figure 3.22 Simulation set up (subject 1)

Injury was predicted probabilistically using injury risk functions. Injury criteria extracted from the literature were used to assess the risk of sustaining specific injuries (Table 3.14). Injury 
risk functions can be seen in previous literature (Chen et al. 2017). The simulation end time for injury predictions was $20 \mathrm{~ms}$ after the time of the first head-vehicle contact.

Table 3.14 Injury criteria

\begin{tabular}{|c|c|c|c|}
\hline Injury criteria & Predicted injury & Injury severity & Reference \\
\hline HIC & Skull fracture & $\begin{array}{l}\text { Moderate } \\
\text { (AIS2+) }\end{array}$ & Kuppa 2004 \\
\hline Chest deflection (lateral, frontal) & Rib fracture & Serious (AIS3+) & $\begin{array}{l}\text { Kuppa et al. 2003, } \\
\text { Kent et al. } 2005\end{array}$ \\
\hline Pelvis deflection & Pelvis fracture & AIS2+ & Gunji et al. 2012 \\
\hline Thigh moment (proximal, middle, distal) & Femur fracture & AIS3+ & Kerrigan et al. 2004 \\
\hline Leg moment (middle) & Tibia/Fibula fracture & AIS2+ & Kerrigan et al. 2004 \\
\hline Upper tibia axial force & Tibia plateau fracture & AIS2+ & $\begin{array}{l}\text { Banglmaier et al. } \\
1999\end{array}$ \\
\hline Knee bending angle and shear displacement & $\begin{array}{l}\text { Knee } \\
\text { rupture }\end{array}$ & AIS2+ & $\begin{array}{l}\text { Ivarsson et al. 2004, } \\
\text { Mo et al. } 2014\end{array}$ \\
\hline $\begin{array}{l}\text { Ankle bending angle and lower tibia axial } \\
\text { load }\end{array}$ & Ankle injury & AIS2+ & Funk et al. 2002 \\
\hline
\end{tabular}

\subsubsection{Results}

\section{$\underline{\text { Response }}$}

Figure 3.23 - Figure 3.27 showed the segmental trajectory, velocity and acceleration for subject 1 and subject 2. In both cases, when decreasing the Young modulus by 10\%, the segmental trajectory, velocity and acceleration didn't show apparent change, which suggested the insensitivity of bone quality on the pedestrian kinematics.

In both cases, when enabling all tissue failures, the knee ligament ruptures, fibula fractures, pelvic fractures and rib fractures were observed. The segmental trajectory, velocity, and acceleration of lower limbs showed slight changes due to knee ligament ruptures and fibula fractures, but the kinematics of pelvis upper body didn’t show apparent change, which suggested enabling tissue failure only has slight local effect (lower extremities), but is insensitive on the pedestrian upper body kinematics. 
Also, when enabling all the tissue failure the differences of head impact conditions were small, including head impact timing, wrap around distance, head impact speed, and head impact angle, as shown in Table 3.15 .
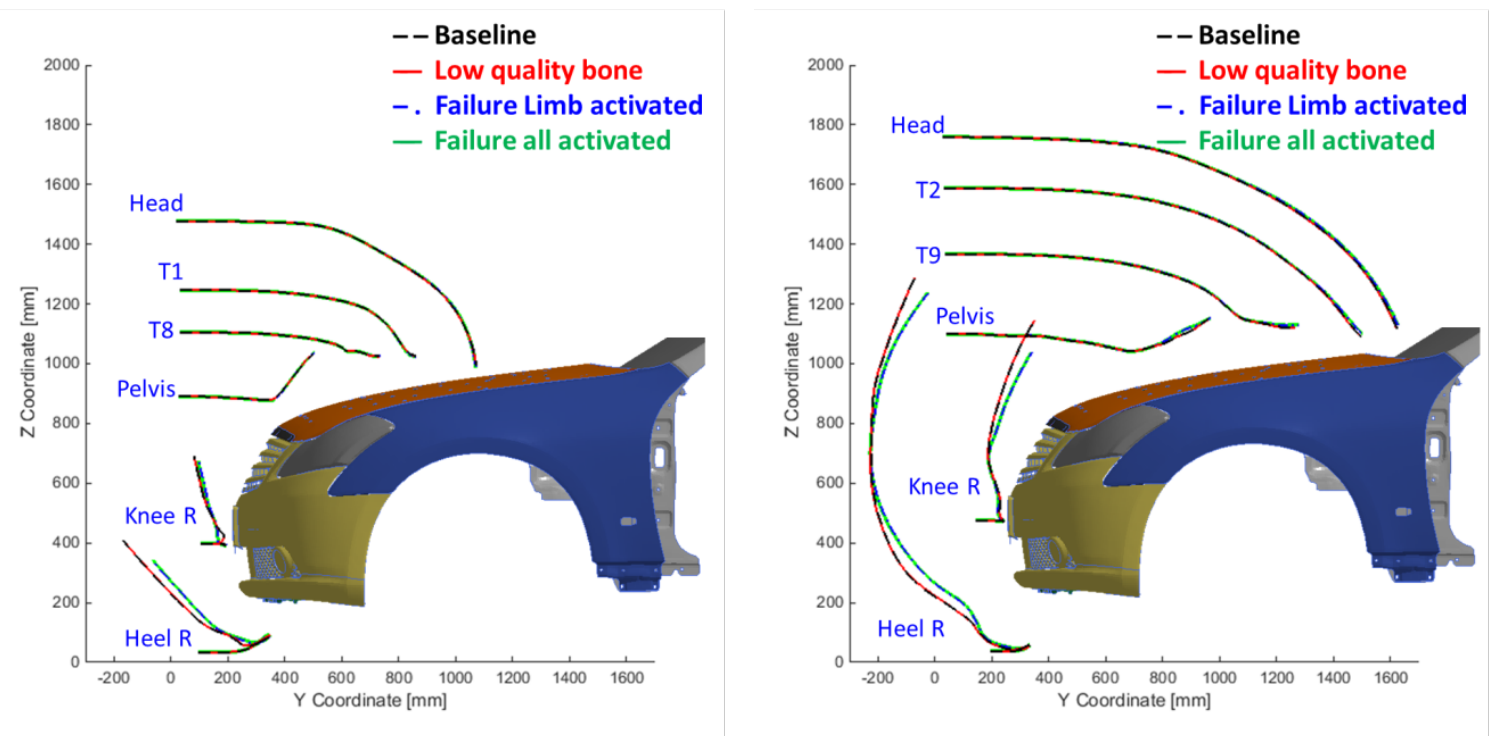

Figure 3.23 Trajectories in vehicle coordinate system (Left - subject 1, Right - subject 2).

Table 3.15 Differences on head impact conditions (between no enabling and all enabled situations)

\begin{tabular}{ccccc}
\hline Case & $\begin{array}{c}\text { Head impact } \\
\text { timing }\end{array}$ & WAD & $\begin{array}{c}\text { Head impact } \\
\text { speed }\end{array}$ & $\begin{array}{c}\text { Head impact } \\
\text { angle }\end{array}$ \\
\hline 1 & $0.4 \mathrm{~ms}$ & $0 \mathrm{~mm}$ & $-0.7 \mathrm{~m} / \mathrm{s}$ & $0.0^{\circ}$ \\
2 & $0.6 \mathrm{~ms}$ & $0 \mathrm{~mm}$ & $0.0 \mathrm{~m} / \mathrm{s}$ & $-0.9^{\circ}$ \\
\hline
\end{tabular}



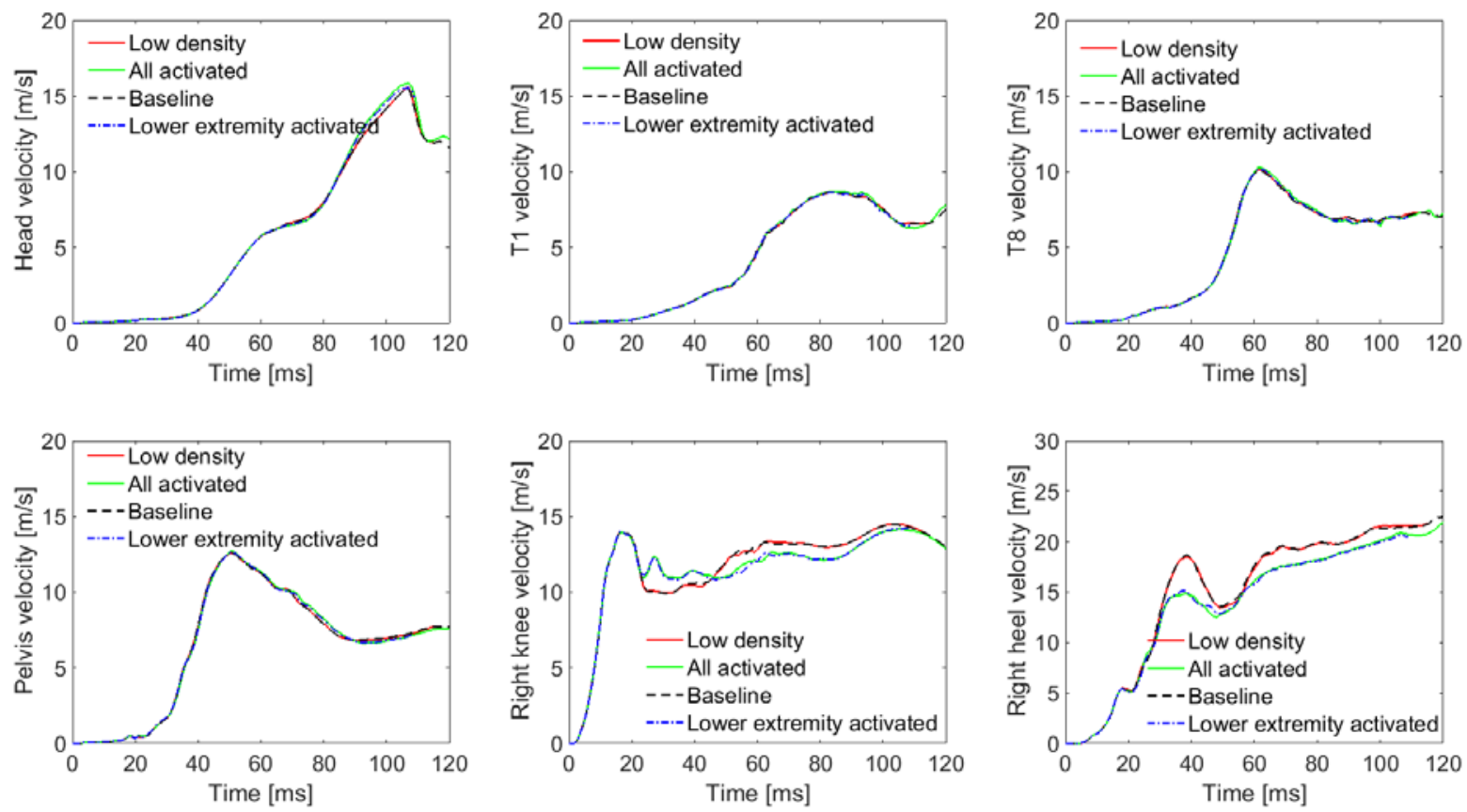

Figure 3.24 Time history of resultant velocity on each body region (subject 1).
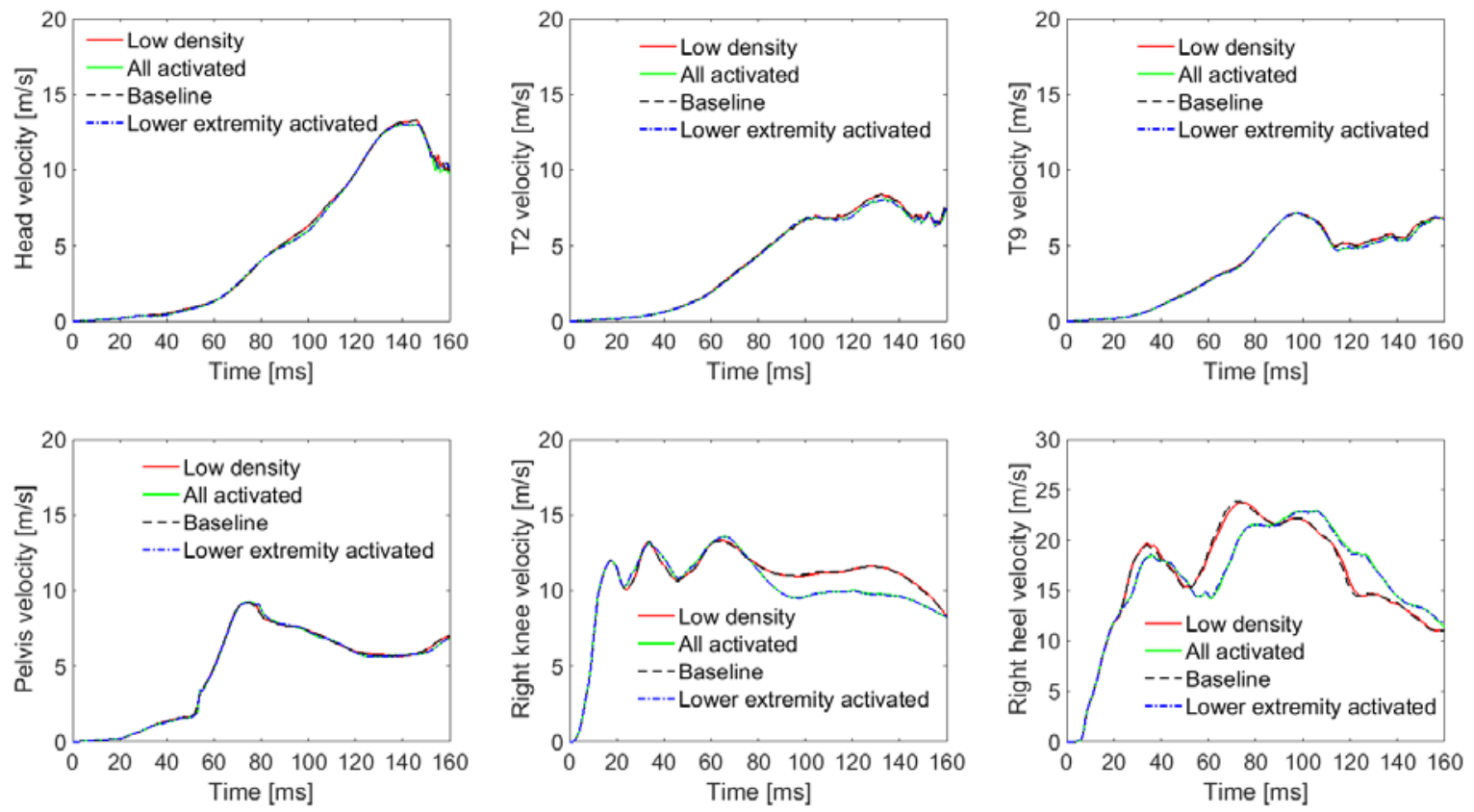

Figure 3.25 Time history of resultant velocity on each body region (subject 2). 

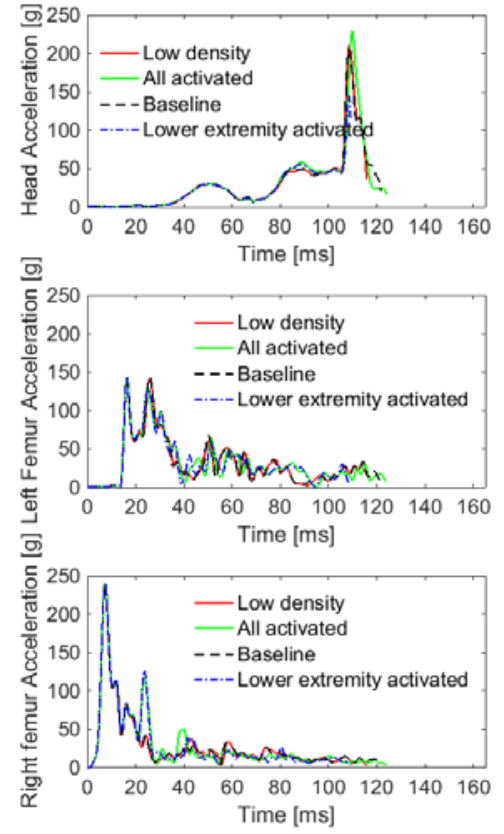
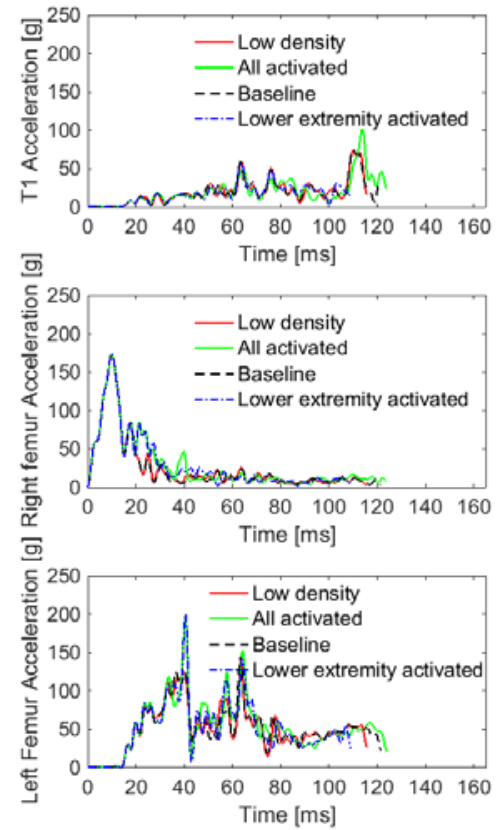
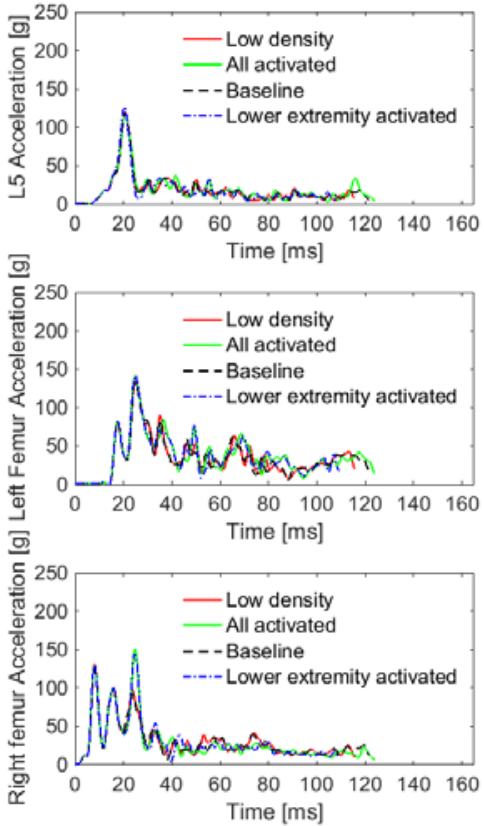

Figure 3.26 Time history of resultant acceleration on each body region (subject 1).
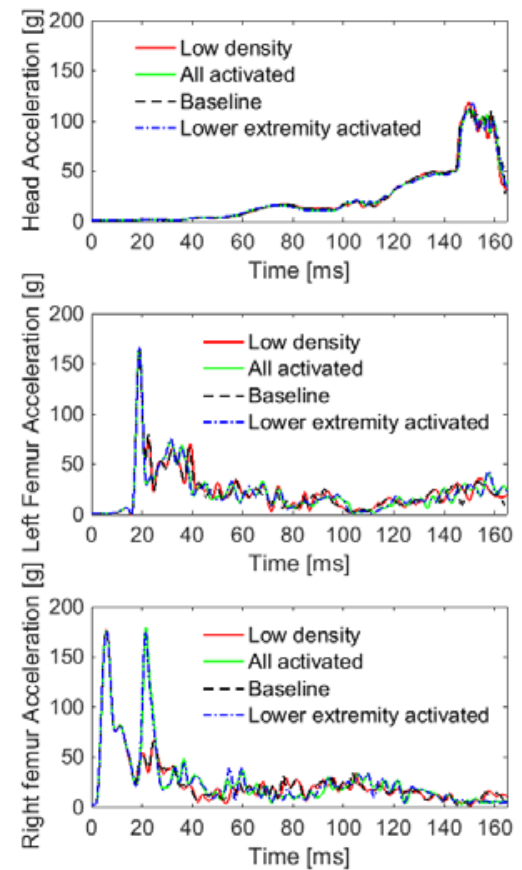
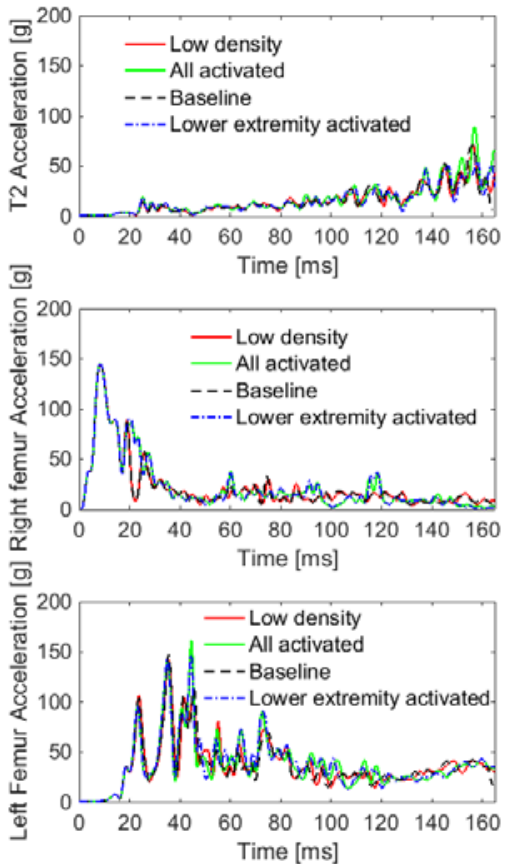
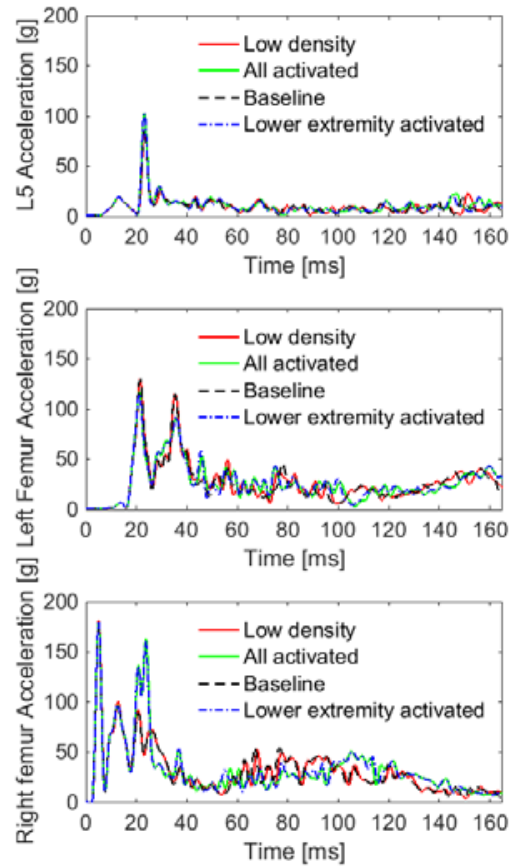

Figure 3.27 Time history of resultant acceleration on each body region (subject 2). 
$\underline{\text { Injury }}$

Table 3.16 - Table 3.17 showed predicted injury of both subjects for baseline simulation, simulation of low quality bone and failure all enabled using probabilistic method (PM). In both cases, when lowering the bone quality or enabling the tissue failure, the whole body injury risk didn't show apparent change, which suggested the insensitivity of bone quality and tissue failure on the pedestrian injury risk.

Table 3.16 Summary of predicted injury of subject 1, for baseline simulation, simulation of low quality bone and failure all enabled using probabilistic method (PM)

\begin{tabular}{|c|c|c|c|c|c|}
\hline Body region & Predicted injury & Baseline & $\begin{array}{c}\text { Low quality } \\
\text { bone }\end{array}$ & $\begin{array}{c}\text { Failure limb } \\
\text { enabled }\end{array}$ & $\begin{array}{c}\text { Failure all } \\
\text { enabled }\end{array}$ \\
\hline Head & Skull Fx. & $\begin{array}{c}74.9 \% \\
\text { (AIS2+) }\end{array}$ & $87.6 \%($ AIS2+) & 74.9\% (AIS2+) & $82.6 \%(\mathrm{AIS} 2+)$ \\
\hline Thorax R & Rib Fx. & $\begin{array}{c}1.1 \% \\
(\mathrm{AIS} 3+)\end{array}$ & $1.1 \%(\mathrm{AIS} 3+)$ & $1.0 \%(\mathrm{AIS3}+)$ & $1.0 \%(\mathrm{AIS}+)$ \\
\hline Thorax L & Rib Fx. & $\begin{array}{c}10.3 \% \\
\text { (AIS3+) }\end{array}$ & $11.5 \%(\mathrm{AIS3}+)$ & $14.2 \%(\mathrm{AIS3}+)$ & $17.8 \%\left(\mathrm{AIS3}^{+}\right)$ \\
\hline Pelvis & Pelvis Fx. & $\begin{array}{l}78.2 \% \\
\text { (AIS2+) }\end{array}$ & $86.1 \%(\mathrm{AIS} 2+)$ & $74.9 \%(\mathrm{AIS} 2+)$ & $78.2 \%(\mathrm{AIS} 2+)$ \\
\hline R Thigh & Femur Fx. & $0 \%\left(\mathrm{AIS3}^{+}\right)$ & $0 \%(\mathrm{AIS3}+)$ & $0 \%(\mathrm{AIS3}+$ ) & $0 \%(\mathrm{AIS} 3+$ ) \\
\hline L Thigh & Femur Fx. & $\begin{array}{l}0.1 \% \\
\text { (AIS3+) }\end{array}$ & $0.0 \%(\mathrm{AIS3}+)$ & $0.1 \%\left(\mathrm{AIS3}^{+}\right)$ & $0.1 \%(\mathrm{AIS}+$ ) \\
\hline R Leg & Tibia/fibula Fx. & $\begin{array}{c}1.5 \% \\
(\mathrm{AIS} 2+)\end{array}$ & $1.4 \%(\mathrm{AIS} 2+)$ & $0.6 \%(\mathrm{AIS} 2+)$ & $1.5 \%(\mathrm{AIS} 2+)$ \\
\hline L Leg & Tibia/fibula Fx. & $\begin{array}{c}0.1 \% \\
\text { (AIS2+) }\end{array}$ & $0.0 \%(\mathrm{AIS} 2+$ ) & $0.1 \%(\mathrm{AIS} 2+)$ & $0.6 \%(\mathrm{AIS} 2+)$ \\
\hline $\begin{array}{l}\text { R Knee } \\
\text { (soft) }\end{array}$ & Ligament Rup. & $\begin{array}{c}100 \% \\
\text { (AIS2+) }\end{array}$ & $100 \%(\mathrm{AIS} 2+)$ & $100 \%(\mathrm{AIS} 2+)$ & $100 \%(\mathrm{AIS} 2+)$ \\
\hline $\begin{array}{l}\text { R Knee } \\
\text { (bone) }\end{array}$ & Tibia plat. Fx. & $\begin{array}{c}0.9 \% \\
\text { (AIS2+) }\end{array}$ & $0.3 \%(\mathrm{AIS} 2+)$ & $0.4 \%(\mathrm{AIS} 2+)$ & $0.4 \%(\mathrm{AIS} 2+$ ) \\
\hline $\begin{array}{l}\text { L Knee } \\
\text { (soft) }\end{array}$ & Ligament Rup. & $\begin{array}{l}100 \% \\
\text { (AIS2+) }\end{array}$ & $100 \%(\mathrm{AIS} 2+)$ & $100 \%(\mathrm{AIS} 2+)$ & $100 \%(\mathrm{AIS} 2+)$ \\
\hline $\begin{array}{l}\text { L Knee } \\
\text { (bone) }\end{array}$ & Tibia plat. Fx. & $\begin{array}{c}1.3 \% \\
\text { (AIS2+) }\end{array}$ & $0.6 \%(\mathrm{AIS} 2+)$ & $0.6 \%(\mathrm{AIS} 2+$ ) & $0.6 \%(\mathrm{AIS} 2+)$ \\
\hline R Ankle & $\begin{array}{c}\text { Ligament Rup., bone } \\
\text { Fx. }\end{array}$ & $\begin{array}{c}0.1 \% \\
\text { (AIS2+) }\end{array}$ & $0.1 \%(\mathrm{AIS} 2+)$ & $0.0 \%(\mathrm{AIS} 2+$ ) & $0.0 \%(\mathrm{AIS} 2+$ ) \\
\hline L Ankle & $\begin{array}{c}\text { Ligament Rup., bone } \\
\text { Fx. }\end{array}$ & $\begin{array}{c}100 \% \\
(\mathrm{AIS} 2+) \\
\end{array}$ & $100 \%(\mathrm{AIS} 2+)$ & $100 \%(\mathrm{AIS} 2+)$ & $100 \%(\mathrm{AIS} 2+$ ) \\
\hline
\end{tabular}


Table 3.17 Summary of predicted injury of subject 2, for baseline simulation, simulation of low quality bone, failure limb enabled, and failure all enabled using probabilistic method (PM)

\begin{tabular}{|c|c|c|c|c|c|}
\hline Body region & Predicted injury & Baseline & $\begin{array}{l}\text { Low quality } \\
\text { bone }\end{array}$ & $\begin{array}{c}\text { Failure limb } \\
\text { enabled }\end{array}$ & $\begin{array}{c}\text { Failure all } \\
\text { enabled }\end{array}$ \\
\hline Head & Skull Fx. & $\begin{array}{l}53.4 \% \\
(\mathrm{AIS} 2+)\end{array}$ & $52.5 \%(\mathrm{AIS} 2+)$ & $52.5 \%(\mathrm{AIS} 2+)$ & $53.8 \%($ AIS2+) \\
\hline Thorax R & Rib Fx. & $81 \%(\mathrm{AIS3}+$ ) & 76.4\% (AIS3+) & 72.3\% (AIS3+) & $78.7 \%(\mathrm{AIS3}+)$ \\
\hline Thorax L & Rib Fx. & $\begin{array}{c}47.8 \% \\
\text { (AIS3+) }\end{array}$ & $62.5 \%(\mathrm{AIS3}+)$ & $52.6 \%(\mathrm{AIS3}+)$ & $59.4 \%\left(\mathrm{AIS3}^{+}\right)$ \\
\hline Pelvis & Pelvis Fx. & $\begin{array}{c}11.2 \% \\
\text { (AIS2+) }\end{array}$ & $11.7 \%(\mathrm{AIS} 2+$ ) & $18.7 \%(\mathrm{AIS} 2+)$ & $17.5 \%(\mathrm{AIS} 2+)$ \\
\hline R Thigh & Femur Fx. & $91 \%(\mathrm{AIS3}+)$ & $89.4 \%$ (AIS3+) & 92.9\% (AIS3+) & $92.1 \%(\mathrm{AIS3}+)$ \\
\hline L Thigh & Femur Fx. & $\begin{array}{c}5.5 \% \\
\text { (AIS3+) }\end{array}$ & $4.9 \%(\mathrm{AIS3}+)$ & $3.5 \%(\mathrm{AIS3}+$ ) & $3.8 \%(\mathrm{AIS} 3+)$ \\
\hline R Leg & Tibia/fibula Fx. & $\begin{array}{c}6.8 \% \\
\text { (AIS2+) }\end{array}$ & $6.9 \%(\mathrm{AIS} 2+)$ & $4.5 \%$ (AIS2+) & $4.6 \%(\mathrm{AIS} 2+)$ \\
\hline L Leg & Tibia/fibula Fx. & $\begin{array}{c}0.3 \% \\
\text { (AIS2+) }\end{array}$ & $0.3 \%(\mathrm{AIS} 2+)$ & $0.3 \%(\mathrm{AIS} 2+)$ & $0.3 \%(\mathrm{AIS} 2+)$ \\
\hline $\begin{array}{l}\text { R Knee } \\
\text { (soft) }\end{array}$ & Ligament Rup. & $\begin{array}{l}100 \% \\
\text { (AIS2+) }\end{array}$ & $100 \%(\mathrm{AIS} 2+)$ & $100 \%(\mathrm{AIS} 2+)$ & $100 \%(\mathrm{AIS} 2+)$ \\
\hline $\begin{array}{l}\text { R Knee } \\
\text { (bone) }\end{array}$ & Tibia plat. Fx. & $\begin{array}{c}0.2 \% \\
(\mathrm{AIS} 2+)\end{array}$ & 13.3\% (AIS2+) & $0.8 \%$ (AIS2+) & $0.8 \%$ (AIS2+) \\
\hline $\begin{array}{l}\text { L Knee } \\
\text { (soft) }\end{array}$ & Ligament Rup. & $\begin{array}{c}100 \% \\
\text { (AIS2+) }\end{array}$ & $100 \%(\mathrm{AIS} 2+)$ & $100 \%$ (AIS2+) & $100 \%(\mathrm{AIS} 2+$ ) \\
\hline $\begin{array}{l}\text { L Knee } \\
\text { (bone) }\end{array}$ & Tibia plat. Fx. & $0 \%(\mathrm{AIS} 2+)$ & $0.4 \%(\mathrm{AIS} 2+)$ & $0.4 \%(\mathrm{AIS} 2+$ ) & $0.4 \%(\mathrm{AIS} 2+)$ \\
\hline R Ankle & $\begin{array}{c}\text { Ligament Rup., bone } \\
\text { Fx. }\end{array}$ & $0 \%(\mathrm{AIS} 2+$ ) & $0 \%(\mathrm{AIS} 2+)$ & $0 \%(\mathrm{AIS} 2+$ ) & $0 \%(\mathrm{AIS} 2+$ ) \\
\hline L Ankle & $\begin{array}{c}\text { Ligament Rup., bone } \\
\text { Fx. }\end{array}$ & $\begin{array}{c}100 \% \\
(\mathrm{AIS} 2+)\end{array}$ & $100 \%(\mathrm{AIS} 2+)$ & $100 \%$ (AIS2+) & $100 \%(\mathrm{AIS} 2+)$ \\
\hline
\end{tabular}

\subsubsection{Conclusion}

The pedestrian kinematics is insensitive to bone quality, while enabling tissue failure only has the local effect on kinematics of severe injured area (lower extremities), but has little effect on the pedestrian upper body kinematics. Also, the pedestrian whole body injury risk didn’t show significant sensitivity on the bone quality or enabling the tissue failure. 


\subsection{Appendix for Chapter 3}

\subsubsection{Anthropometry Parameters and Positioning}

Table 3.18 Detailed anthropometry parameters (errors with respect to PMHS are in bracket).

\begin{tabular}{ccccccccccc}
\hline & \multicolumn{3}{c}{ Height $(\mathbf{m m})$} & \multicolumn{7}{c}{ Mass (kg) } \\
\cline { 2 - 11 } PMHS 1 & Knee & Pelvic & Standing & Body & Head & Torso & Pelvis & Upper limb & Thigh & Leg \\
\cline { 2 - 11 } B97 & 891 & 1520 & 72.6 & - & - & - & - & - & - \\
Baseline 1 & $475(78)$ & $1021(130)$ & $1723(203)$ & $77.0(4.4)$ & 4.3 & 23.8 & 7.7 & 3.0 & 6.5 & 4.3 \\
Morphed 1 & $392(-5)$ & $891(0)$ & $1520(0)$ & $70.3(-2.3)$ & 4.9 & 21.7 & 6.9 & 3.3 & 5.1 & 3.5 \\
PMHS 2 & 516 & 1095 & 1838 & 114 & - & - & - & - & - & - \\
Baseline 2 & $490(-26)$ & $1023(-72)$ & $1727(-111)$ & $77.0(-37)$ & 4.3 & 23.8 & 7.7 & 3.0 & 6.5 & 4.3 \\
Morphed 2 & $502(-14)$ & $1092(-3)$ & $1832(-6)$ & $118(4)$ & 5.2 & 40.0 & 14.4 & 5.0 & 11.2 & 4.8 \\
\hline
\end{tabular}

- Not reported

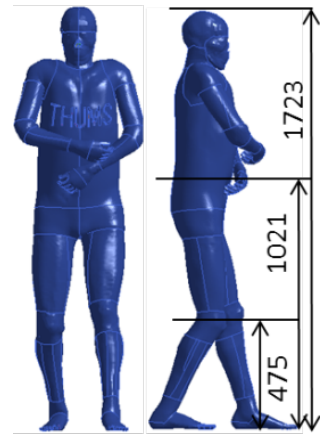

AM50-1

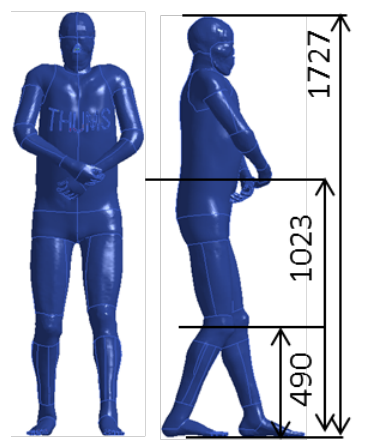

AM50-2

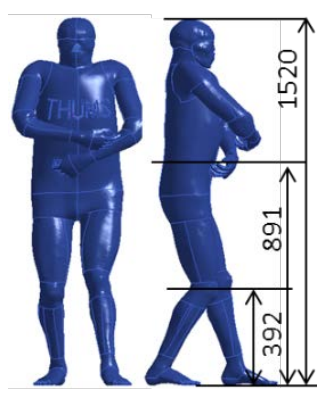

Morphed 1

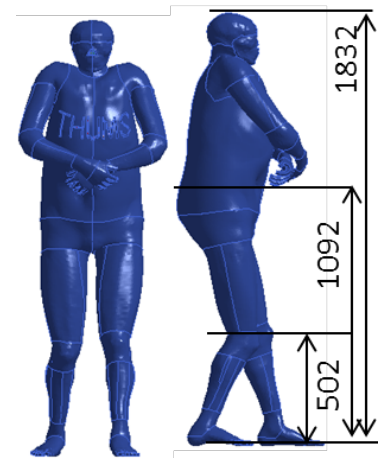

Morphed 2

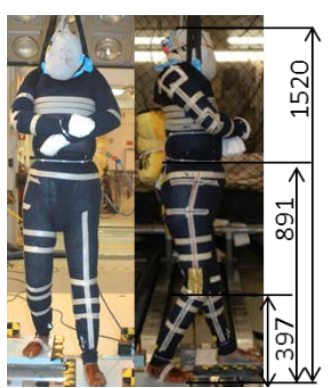

PMHS 1

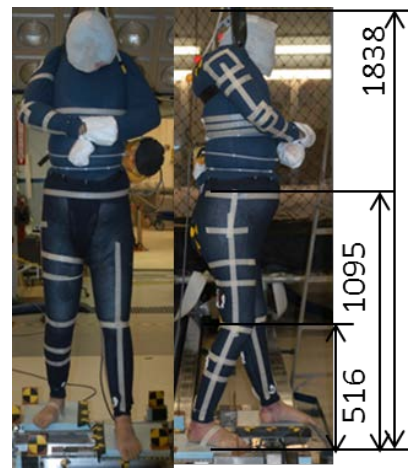

PMHS 2

Figure 3.28 Initial positions of PMHS, morphed model and AM50 model 


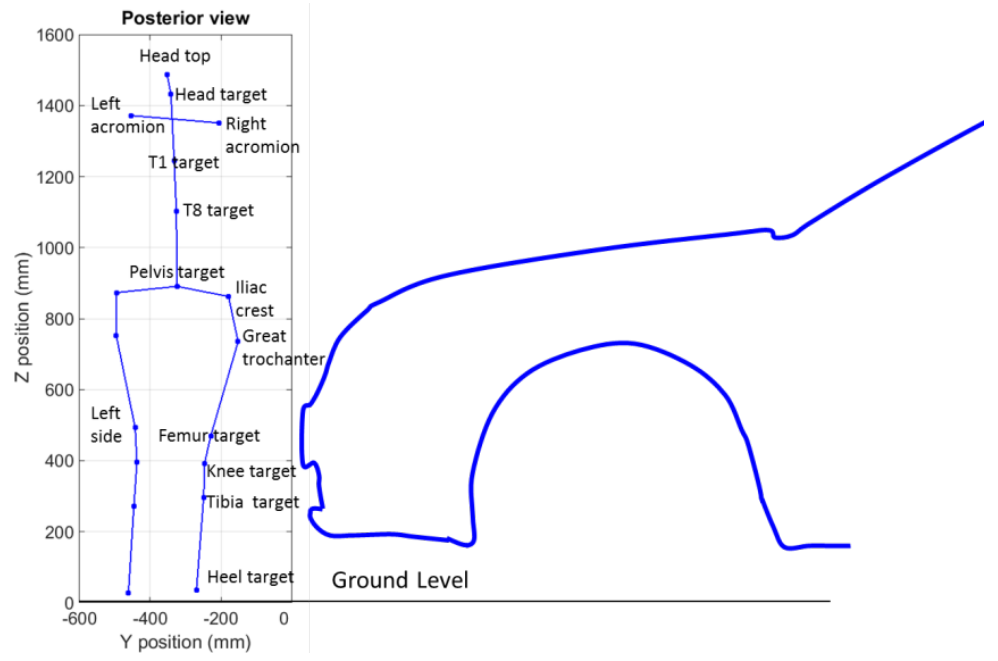

Figure 3.29 Anatomical landmarks and schematic of the centerline cross-section of the mid-size sedan (Subit et al. 2008).

\subsubsection{Trajectory}
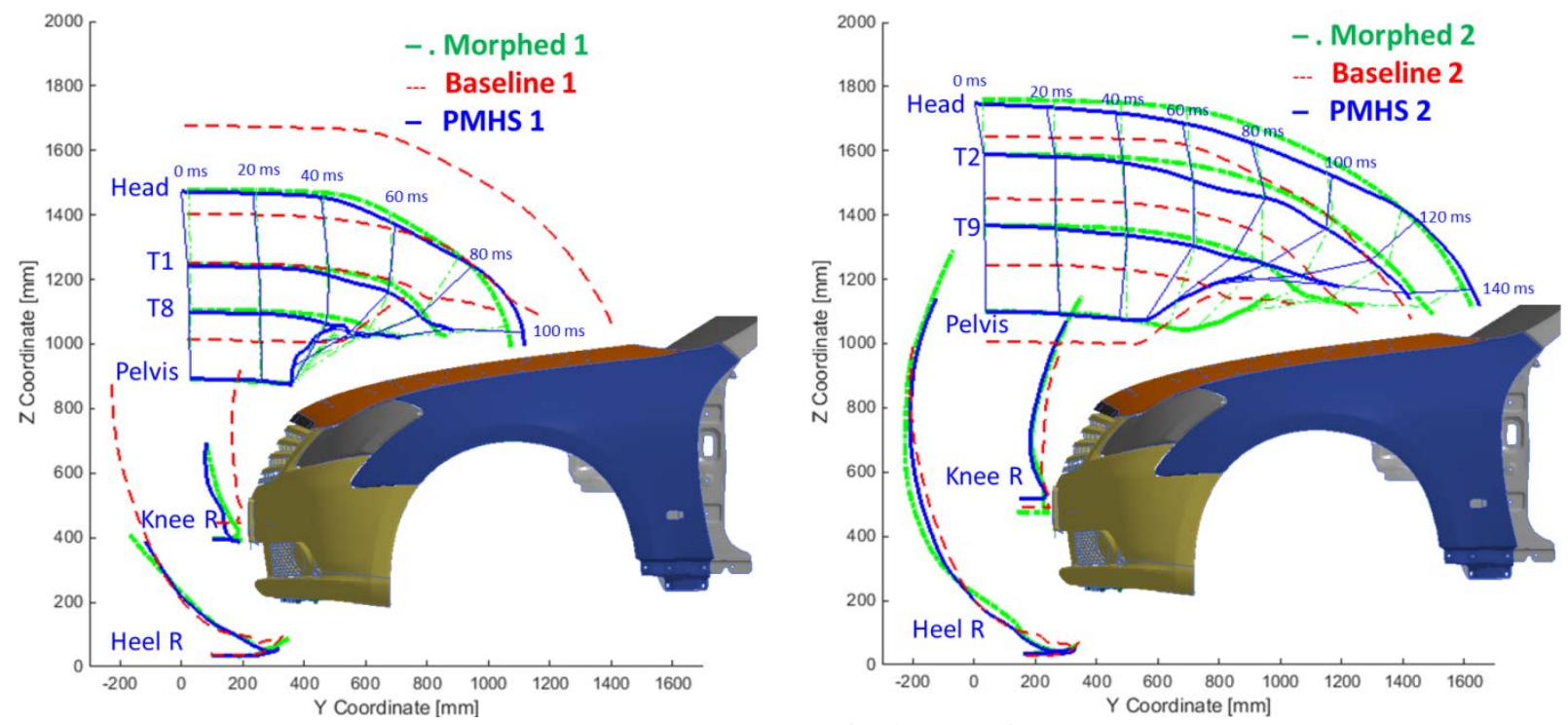

Figure 3.30 Trajectories in vehicle coordinate system 


\subsubsection{Strain}

Table 3.19 Strain gauge locations

\begin{tabular}{cccccccc}
\hline Gauge Number (PMHS 1) & 5 & 6 & 7 & 8 & 9 & 10 & 11 \\
Bone/Face & $\begin{array}{c}\text { LF/Post- } \\
\text { Med }\end{array}$ & $\begin{array}{c}\text { LF/Lat- } \\
\text { Post }\end{array}$ & $\begin{array}{c}\text { LF/Ant- } \\
\text { Med }\end{array}$ & $\begin{array}{c}\text { LF/Ant- } \\
\text { Lat }\end{array}$ & $\begin{array}{c}\text { RT/Pos- } \\
\text { Med }\end{array}$ & $\begin{array}{c}\text { RT/Ant- } \\
\text { Med }\end{array}$ & RT/Ant \\
\hline Gauge Number (PMHS 2) & 3 & 4 & 5 & 8 & 9 & 10 & 11 \\
Bone/Face & $\begin{array}{c}\text { LF-Ant } \\
\text { Med }\end{array}$ & $\begin{array}{c}\text { LF/Ant- } \\
\text { Lat }\end{array}$ & $\begin{array}{c}\text { RF/Post- } \\
\text { Lat }\end{array}$ & $\begin{array}{c}\text { RF/Ant- } \\
\text { Lat }\end{array}$ & $\begin{array}{c}\text { RT/Post } \\
\text { RT/Ant- } \\
\text { Med }\end{array}$ & $\begin{array}{c}\text { RT/Ant } \\
\text { RT/Ant- } \\
\text { Lat }\end{array}$ \\
\hline
\end{tabular}

Bone: LF-Left Femur, RF-Right Femur, RT-Right Tibia $\quad$ Face: Ant-Anterior, Med-Medial, Lat-Lateral, Post-Posterior
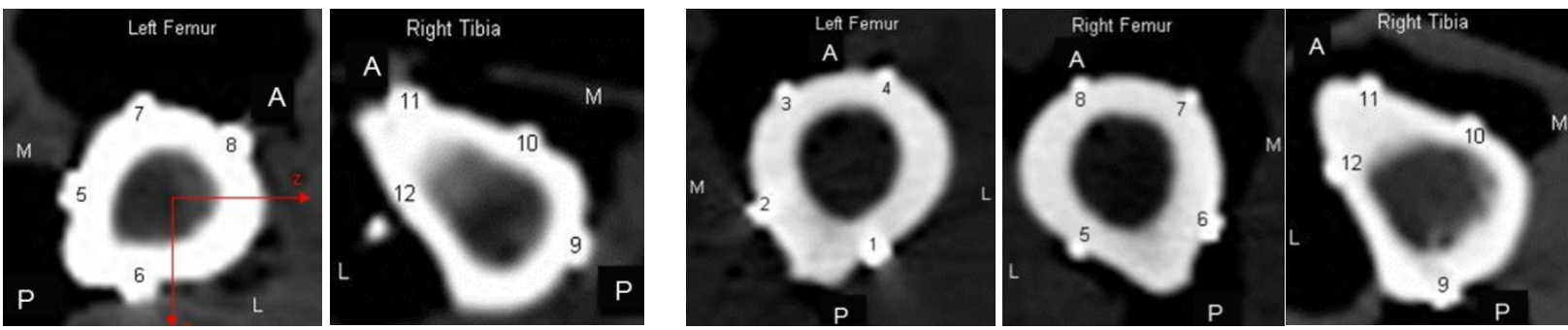

Figure 3.31 Strain gauge locations (PMHS 1) Figure 3.32 Strain gauge locations (PMHS 2)
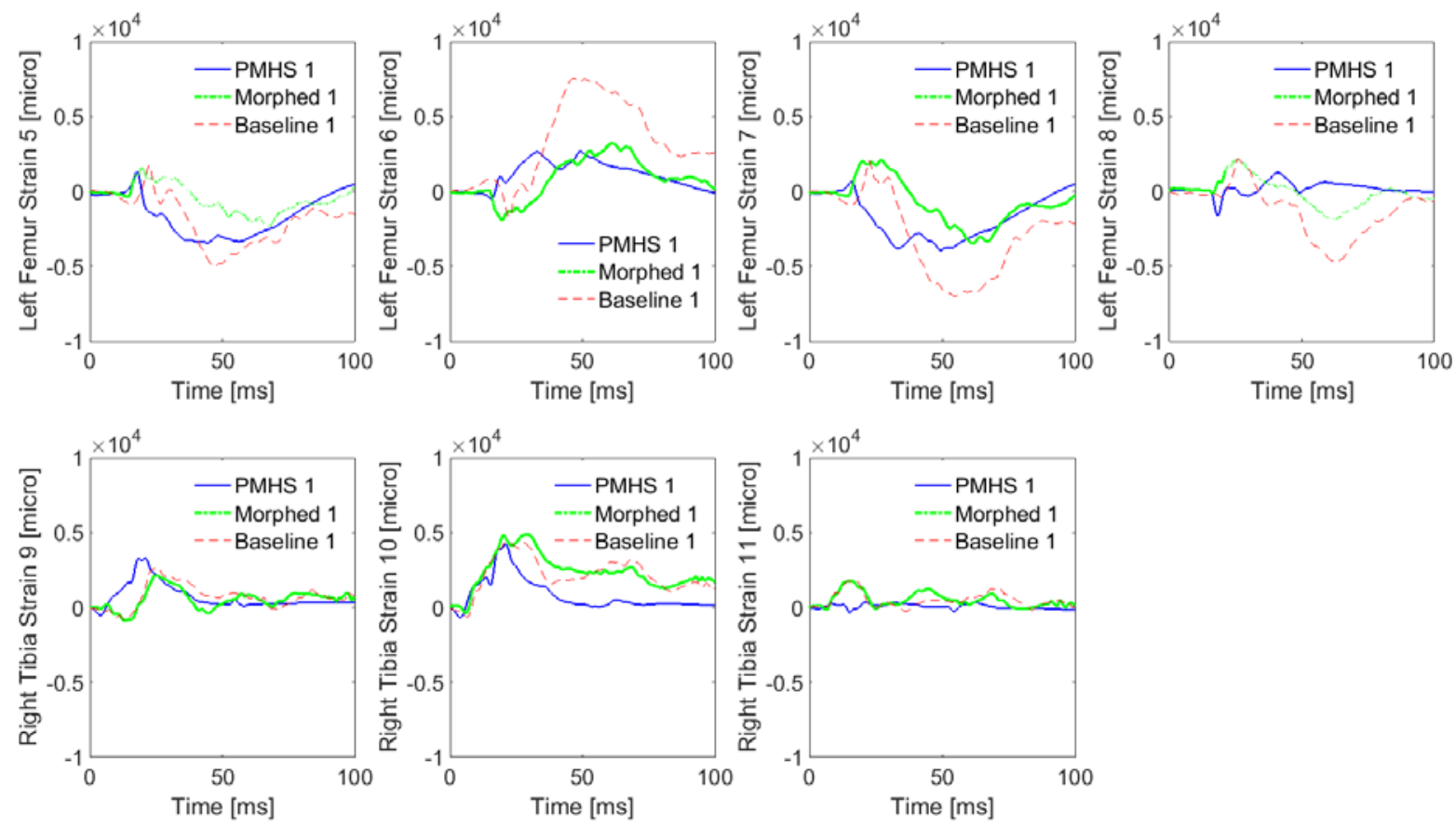

Figure 3.33 Time history of lower extremity strains (positive value is tension) (subject 1). 

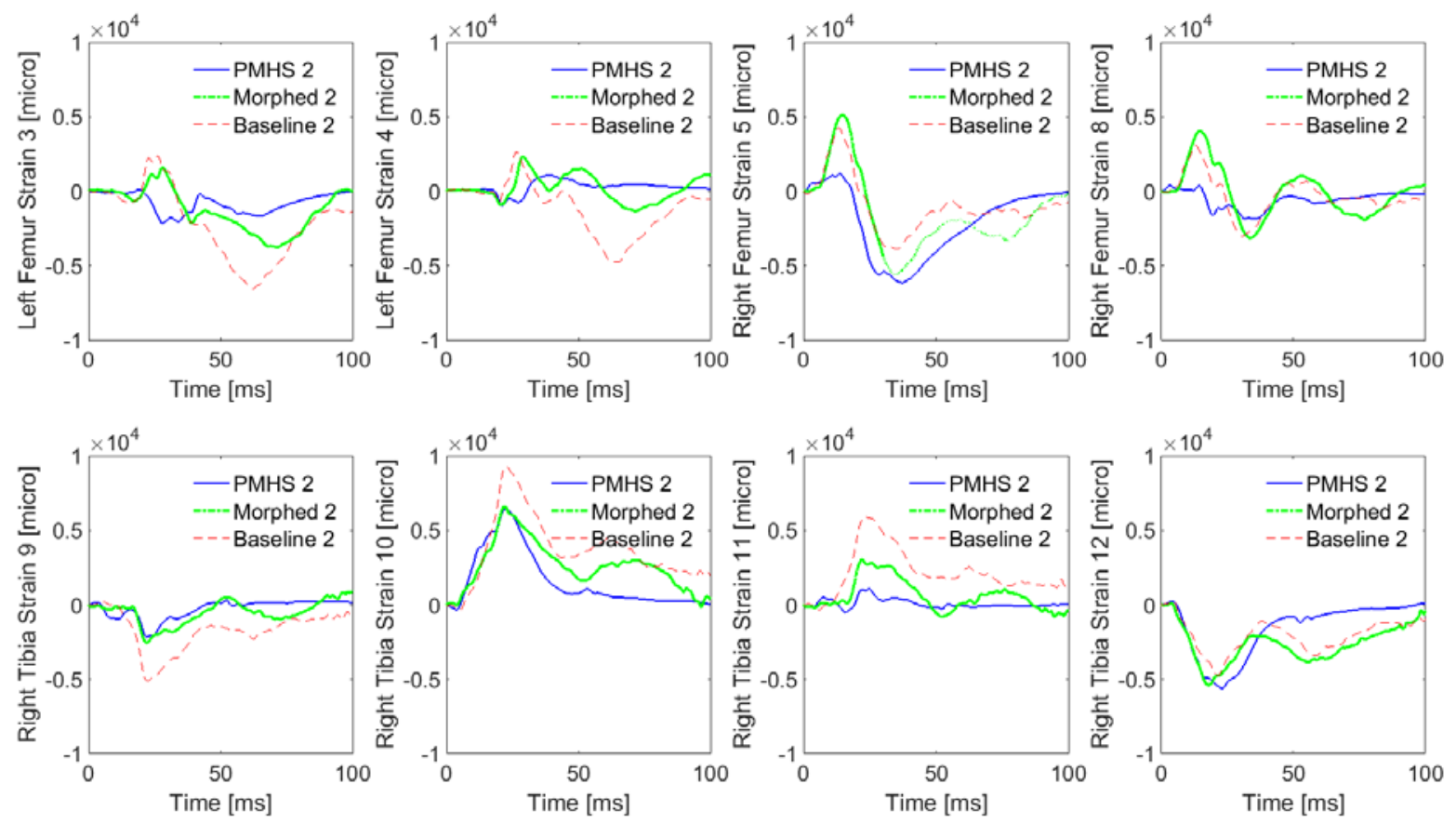

Figure 3.34 Time history of lower extremity strains (subject 2) 


\subsubsection{PMHS Injury}
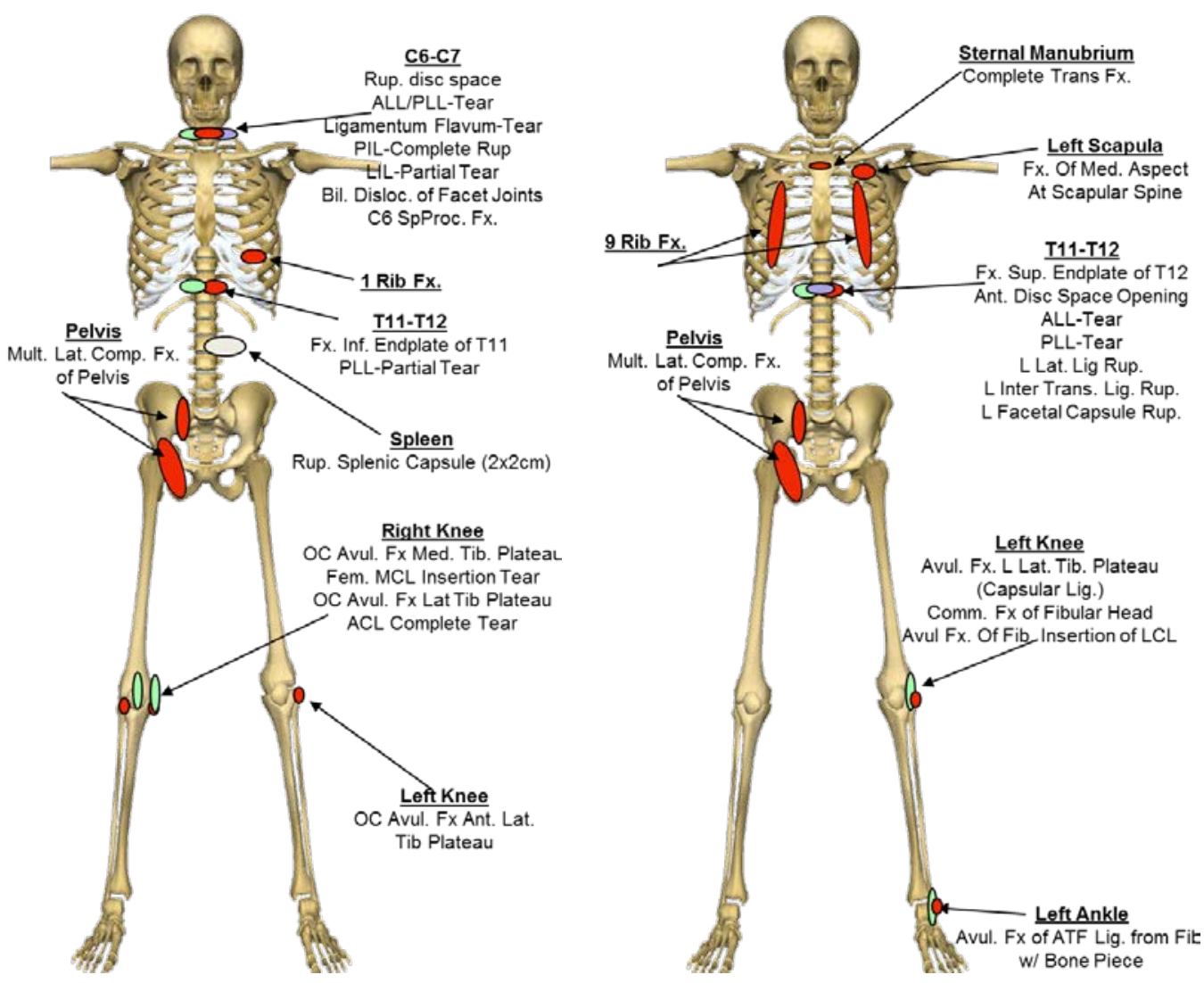

Ant.-Anterior ALL—Anterior Longitudinal Ligament ATF-Anterior Talo-Fibular (Ligament) Avul.—Avulsion Bil.—Bilateral CC—Costochondral as in Costrochondral Junction COD—Cause of Death Comp.—Compression Com.-Comminuted Disloc.Dislocation FacJnt.—Facet Joint Fem.-Femur Fib.—Fibula Fx.—Fracture Inf.-Inferior L. —Left Lat.—Lateral Lig.— Ligament LigFlav.—Ligamentum Flavum LIL—Lateral Interspinous Ligaments $\quad$ Med.—Medial $\quad$ MCL—Medial Collateral Ligament Mult.-Multiple Obl.—Oblique OC—Osteochondral $\quad$ PIL—Posterior Interspinous Ligaments PLL—Posterior Longitudinal Ligament Pos.-Posterior R.-Right Rup.-Rupture SpProc.-Spinous Process SC—Sterno-Chondral SI—Sacro-Illiac Sup.-Superior Tib.-Tibia Trans.-Transverse 

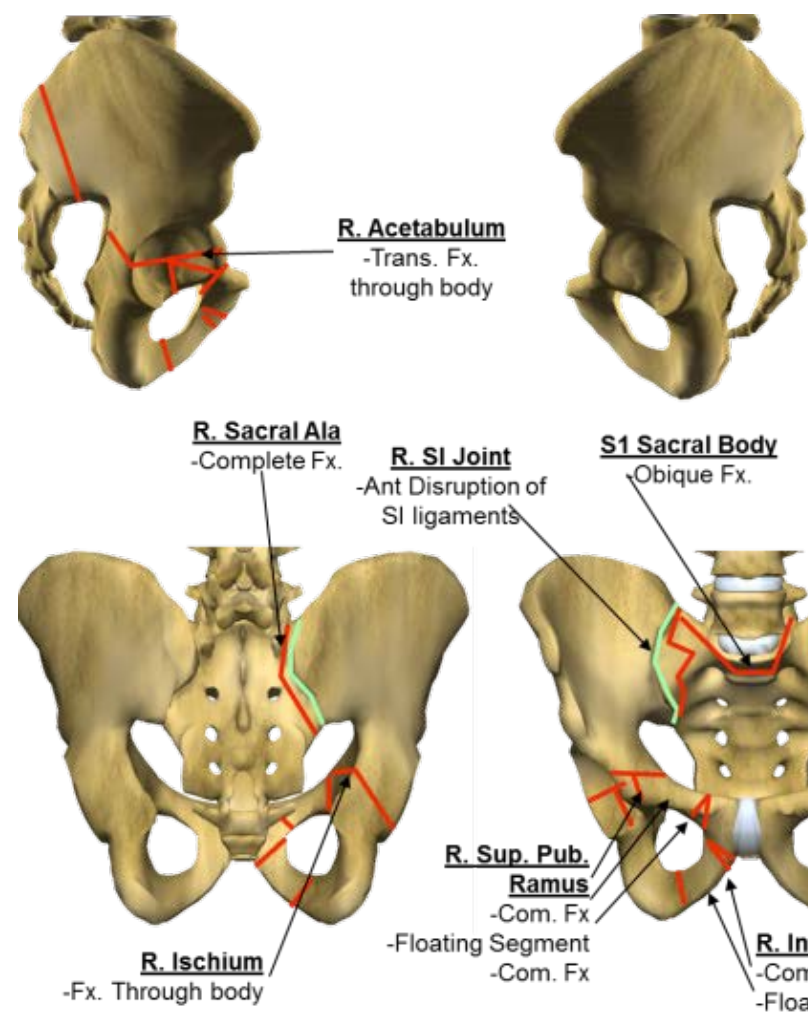

$\frac{\text { R. SI Joint }}{\text { Ant Disruption of }}$

SI ligaments

\section{S1 Sacral Body} Obique $\mathrm{Fx}$.
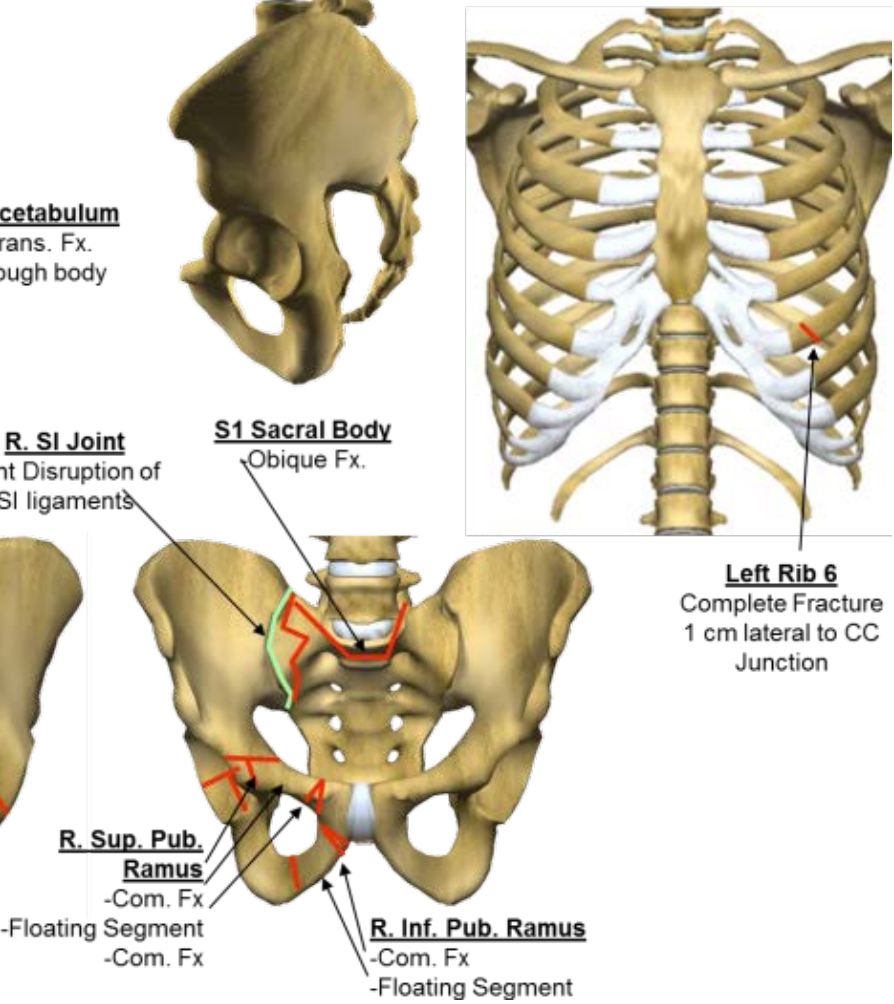

Left Rib 6

Complete Fracture

$1 \mathrm{~cm}$ lateral to $\mathrm{CC}$ Junction
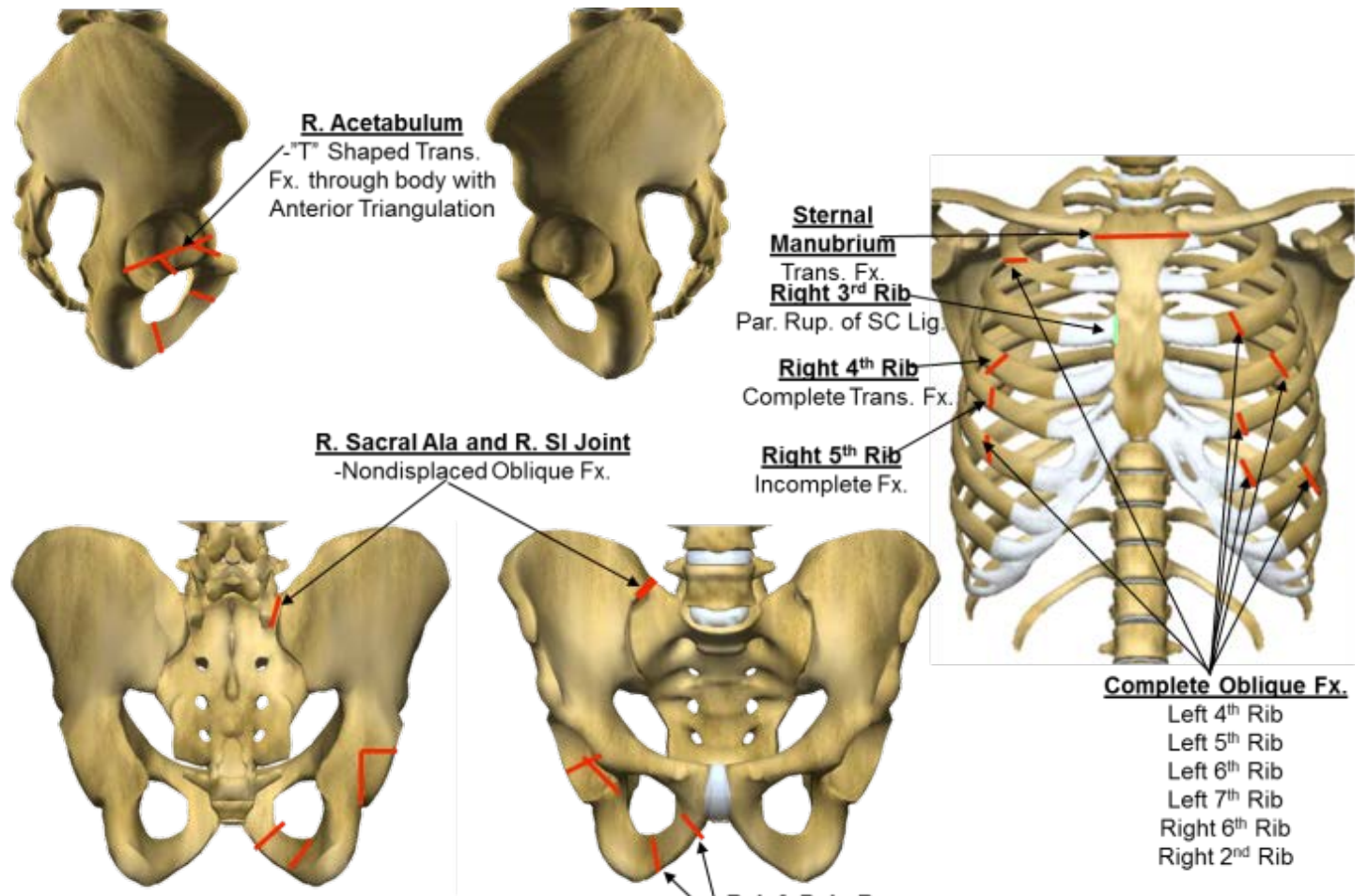
Oblique Fx.
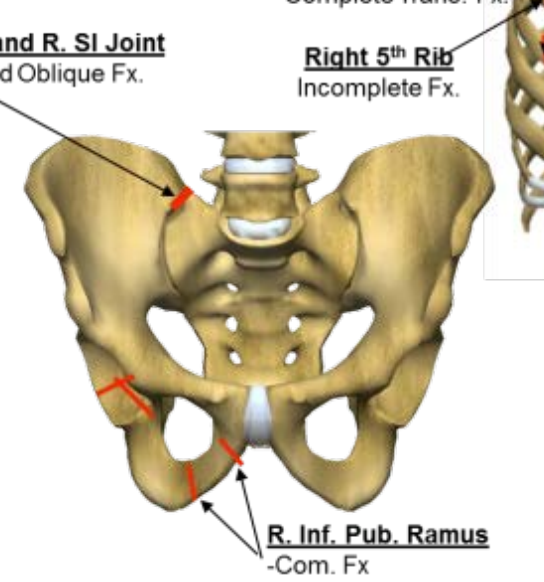

Figure 3.35 Experimental Subjects injury diagrams (left-PMHS1, right-PMHS 2) (Subit et al. 2008) 


\subsubsection{Injury Risk Functions Used for the Probabilistic Method (PM)}

The injury risks of the head, thorax, pelvis and lower extremities were calculated based on the injury risk functions derived from component cadaver tests. The HIC was used to predict head injury (AIS 2+) (Kuppa 2004). Chest deflection was used to predict the rib fracture (AIS $3+$ when more than 3 rib fractures). The injury risk function of the lateral impact direction is based on the normalized average half chest deflection, which was determined as the maximum of the computed normalized average band deflection (3 points computed on each band) for the upper (T4) and lower (T8) chest band (Kuppa et al. 2003); while for the frontal direction it is based on the normalized average deflection of upper and lower chest band (the percent of initial anterior-posterior chest depth) (Kent et al. 2005). The ages were set as 62 years old to represent two cadavers. The combined injury probability of rib fractures was calculated from lateral and frontal injury risks. Pelvis deflection was used to predict the pelvis fracture (Gunji et al. 2012). Thigh moments (proximal and distal thigh, middle thigh) and leg moment (middle leg) were used to predict the femur fracture and tibia fracture (Kerrigan et al. 2004). The upper tibia axial force was used to predict the tibia plateau fracture (Banglmaier et al. 1999). The valgus or varus bending angle of the knee (Ivarsson et al. 2004) was used to predict knee ligament ruptures. Also, lateral bending angle $\left(16^{\circ}\right)$ and shearing displacement (14 mm) for knee ligament rupture (Mo et al. 2014) were applied as a reference, and the measurement method introduce by Li et al. (2015) was used. The inversion/eversion bending angle and lower tibia axial load were used to predict ankle injury (Funk et al. 2002), and the bending angle measured is the rotation angle of the calcaneus relative to the tibia in the coronal plane. For the shoulder, the $2 \mathrm{KN}$ threshold of clavicle axial compressive force (Zhang et al. 2013) was used to predict clavicle fracture. 
Table 3.20 Injury risk functions

\begin{tabular}{|c|c|c|}
\hline Measurement & Predicted injury & Risk function \\
\hline \multirow{2}{*}{ HIC } & \multirow{2}{*}{ Head injury } & \multirow{2}{*}{$P(A I S 2+)=\phi\left(\frac{\ln (H I C 15)-6.96352}{0.84664}\right)$} \\
\hline & & \\
\hline Chest deflection (Lateral) & Rib fracture & $P_{L}(A I S 3+)=\frac{1}{1+0(9.02937-0.03705 * a g e-368232 * \text { norm avg half defl })}$ \\
\hline & & $1+e^{(9.02937-0.03705 * \text { age }-36.8232 * \text { norm.avg.half defl })}$ \\
\hline \multirow[t]{3}{*}{ Chest deflection (Frontal) } & Rib fracture & $P_{F}(A I S 3+)=\frac{1}{1}$ \\
\hline & & $1+e^{(0.05-10.130}$ \\
\hline & Rib fracture (total) & $P(A I S 3+)=P_{L}+P_{F}-P_{L} P_{F}$ \\
\hline Pelvis deflection & Pelvis fracture & $P(A I S 2+)=1-e^{\left(-e^{(\ln (X)-1.116) / 0.292}\right)}$ \\
\hline Thigh moment (Proximal, distal) & Femur fracture & $P(A I S 3+)=1-e^{\left(-e^{6.24242 * \ln (M)-36.4101-0.90267}\right)}$ \\
\hline Thigh moment (middle) & Femur fracture & $P(A I S 3+)=1-e^{\left(-e^{6.24242 * \ln (M)-36.4101-0.90267-1.144850}\right)}$ \\
\hline Leg moment (middle) & Tibia Fibula fracture & $P(A I S 2+)=1-e^{\left(-e^{5.69112 * \ln (M)-33.05211}\right)}$ \\
\hline Upper tibia axial force & Tibia plateau fracture & $P(A I S 2+)=\frac{1}{1+0(0.5204-0.8189 * F+0.0686 * \text { mass })}$ \\
\hline & & $1+e^{(0.5204-0.8189 * F+0.0686 * m a s s)}$ \\
\hline Knee lateral bending angle & Knee ligament rupture & $P(A I S 2+)=1-e^{\left(-e^{10.7034 * \ln (\alpha)-28.541198}\right)}$ \\
\hline $\begin{array}{l}\text { Ankle lateral bending angle and } \\
\text { tibia axial force }\end{array}$ & Ankle injury & $P(A I S 2+)=1-e^{\left(-e^{4.94 * \ln (\beta)-17.7-0.5 * F}\right)}$ \\
\hline
\end{tabular}

\subsubsection{Injury Prediction}

Table 3.21 Summary of predicted injury for PMHS 1 and Morphed 1 using probabilistic (PM) and deterministic method (DM) (Model prediction inconsistent with PMHS are bolded and underlined).

\begin{tabular}{|c|c|c|c|c|c|c|}
\hline \multirow{2}{*}{ Body region } & \multirow{2}{*}{ Predicted injury } & \multirow{2}{*}{ PMHS 1} & \multicolumn{2}{|c|}{ Morphed 1} & \multicolumn{2}{|c|}{ Baseline 1 } \\
\hline & & & PM & DM & PM & DM \\
\hline Head & Skull Fx. & No & $74.9 \%$ (AIS2+) & No & 80.8\% (AIS2+) & No \\
\hline Neck (soft) & Ligament Rup. & C6-C7 all lig. Rup. & Not eva. & All Lig. Rup. & Not eva. & All Lig. Rup. \\
\hline Neck (bone) & Vertebrae Fx. & C6 SpProc.Fx. & Not eva. & $\underline{\text { No }}$ & Not eva. & $\underline{\text { No }}$ \\
\hline Thorax R & Rib Fx. & No & $1.1 \%(\mathrm{AIS3}+)$ & $\overline{\text { No }}$ & $11.8 \%\left(\mathrm{AIS3}^{+}\right)$ & $\overline{\mathrm{No}}$ \\
\hline Thorax L & Rib Fx. & Rib 6 Fx. & $10.3 \%(\mathrm{AIS} 3+)$ & Rib 7 Fx. & $1.1 \%(\mathrm{AIS} 3+)$ & No \\
\hline Thorax M & Sternum Fx. & No & Not eva. & No & Not eva. & No \\
\hline Shoulder R & Clav/scap Fx. & No & $0 \%(\mathrm{AIS} 2+)$ & Scap Fx. & $0 \%(\mathrm{AIS} 2+)$ & Scap Fx. \\
\hline Shoulder L & Clav/scap fx. & No & $0 \%(\mathrm{AIS} 2+$ ) & No & $0 \%(\mathrm{AIS} 2+$ ) & No \\
\hline Spine (soft) & Ligament Rup. & T11-12 PLL torn & Not eva. & All Lig. Rup. & Not eva. & No \\
\hline Spine (bone) & Vertebrae Fx. & T11 Endplate inf. Fx. & Not eva. & No & Not eva. & No \\
\hline Pelvis R & Pelvis Fx. & Mult .Lat. Fx. & \multirow{2}{*}{$78.8 \%$ (AIS2+) } & Pubic bone Fx. & \multirow{2}{*}{$\underline{41 \%(\text { AIS2+) }}$} & Acetabulum pubic bone \\
\hline Pelvis L & Pelvis Fx. & No & & Acetabulum Fx. & & No \\
\hline R Thigh & Femur Fx. & No & $0 \%(\mathrm{AIS} 3+$ ) & $\overline{\text { No }}$ & $1.7 \%(\mathrm{AIS} 3+$ ) & $\underline{\text { Femur }}$ \\
\hline L Thigh & Femur Fx. & No & $0.1 \%(\mathrm{AIS3}+$ ) & No & $4.3 \%(\mathrm{AIS} 3+$ ) & $\overline{\text { Femur }}$ \\
\hline R Leg & Tibia/fibula Fx. & No & $1.5 \%(\mathrm{AIS} 2+$ ) & No & $3.1 \%(\mathrm{AIS} 2+$ ) & No \\
\hline L Leg & Tibia/fibula Fx. & No & $0.1 \%(\mathrm{AIS} 2+$ ) & Fib. Fx. & $6.2 \%(\mathrm{AIS} 2+)$ & Fib. Fx. \\
\hline R Knee (soft) & Ligament Rup. & MCL ACL Rup. & $100 \%$ (AIS2+) & MCL ACL Rup. & $100 \%$ (AIS2+) & MCL ACL Rup. \\
\hline R Knee (bone) & Tibia plat. Fx. & Tib.Plat. Fx. (Ant Lat) & $\underline{0.9 \%(\text { AIS2+) }}$ & No & $\underline{1.1 \%(\text { AIS2+) }}$ & No \\
\hline L Knee (soft) & Ligament Rup. & No & $100 \%(\mathrm{AIS} 2+)$ & LCL ACL_Rup. & $100 \%$ (AIS2+) & LCL Rup. \\
\hline L Knee (bone) & Tibia plat. Fx. & Tib.Plat Fx. (LCL) & $1.3 \%$ (AIS2+) & No & $1.3 \%$ (AIS2+) & No \\
\hline R Ankle (soft) & Ligament Rup. & No & \multirow{2}{*}{$0.1 \%(\mathrm{AIS} 2+)$} & No & \multirow{2}{*}{$0 \%(\mathrm{AIS} 2+$ ) } & CFL PTFL \\
\hline R Ankle(bone) & Calcaneus/Talus Fx. & No & & No & & No \\
\hline L Ankle (soft) & Ligament Rup. & No & \multirow{2}{*}{$\underline{100 \%(\mathrm{AIS} 2+)}$} & CFL ATFL Rup. & \multirow{2}{*}{$\underline{99.8 \%}$} & CFL ATFL PTFL Rup. \\
\hline L Ankle (bone) & Calcaneus/Talus Fx. & No & & No & & No \\
\hline
\end{tabular}


Table 3.22 Summary of predicted injury for PMHS 2 and Morphed 2 using probabilistic (PM) and deterministic method (DM) (Model prediction inconsistent with PMHS are bolded and underlined).

\begin{tabular}{|c|c|c|c|c|c|c|}
\hline \multirow{2}{*}{ Body region } & \multirow{2}{*}{ Predicted injury } & \multirow{2}{*}{ PMHS 2} & \multicolumn{2}{|c|}{ Morphed 2} & \multicolumn{2}{|r|}{ Baseline 2} \\
\hline & & & $\mathbf{P M}$ & DM & $\mathbf{P M}$ & DM \\
\hline Head & Skull Fx. & No & 53.4\% (AIS2+) & No & 95.7\% (AIS2+) & Skull \\
\hline Neck (soft) & Ligament Rup. & No & Not eva. & All Lig. Rup. & Not eva. & All Lig. Rup. \\
\hline Neck (bone) & Vertebrae Fx. & No & Not eva. & No & Not eva. & $\mathrm{C} 1$ \\
\hline Thorax R & Rib Fx. & Rib 2-6 Fx. & $81 \%(\mathrm{AIS3}+)$ & Rib 3 Fx. & $\underline{7.5 \%(\mathrm{AIS} 3+)}$ & No \\
\hline Thorax L & Rib Fx. & Rib 4-7 Fx. & $47.8 \%$ (AIS3+) & Rib 3 Fx. & $0.1 \%$ (AIS3+) & $\frac{N o}{\text { No }}$ \\
\hline Thorax M & Sternum Fx. & Sternal Fx. & Not eva. & No & Not eva. & No \\
\hline Shoulder R & Clav/scap Fx. & No & $0 \%(\mathrm{AIS} 2+$ ) & Clav. Fx. & $0 \%(\mathrm{AIS} 2+)$ & Scap. Fx. \\
\hline Shoulder L & Clav/scap fx. & Scap. Fx. & $0 \%$ (AIS2+) & Scap. Fx. & $0 \%(\mathrm{AIS} 2+)$ & No \\
\hline Spine (soft) & Ligament Rup. & T11-12 all Lig. Rup. & Not eva. & ALL Rup. & Not eva. & No \\
\hline Spine (bone) & Vertebrae Fx. & T12 Sup Endplate Fx. & Not eva. & T3 Fx. & Not eva. & $\underline{\underline{\text { No }}}$ \\
\hline Pelvis R & Pelvis Fx. & Mult.Lat.Comp.Fx. & \multirow{2}{*}{$\underline{11.2 \%(\text { AIS2+) }}$} & Acetabulum Fx. & \multirow{2}{*}{$32.7 \%$ (AIS2+) } & Acetabulum pubic bone Fx. \\
\hline Pelvis L & Pelvis Fx. & No & & No & & No \\
\hline R Thigh & Femur Fx. & No & $91 \%(\mathrm{AIS} 3+)$ & No & $4.1 \%(\mathrm{AIS3}+)$ & Femur \\
\hline L Thigh & Femur Fx. & No & $5.5 \%(\mathrm{AIS} 3+)$ & No & $2.9 \%(\mathrm{AIS} 3+$ ) & Femur \\
\hline R Leg & Tibia/fibula Fx. & No & $6.8 \%(\mathrm{AIS} 2+$ ) & No & $3.1 \%(\mathrm{AIS} 2+$ ) & No \\
\hline L Leg & Tibia/fibula Fx. & No & $0.3 \%(\mathrm{AIS} 2+$ ) & Fib. Fx. & $6 \%(\mathrm{AIS} 2+)$ & Fib. Fx. \\
\hline R Knee (soft) & Ligament Rup. & No & $100 \%($ AIS2+) & MCL ACL Rup. & $100 \%($ AIS2+) & MCL ACL Rup. \\
\hline R Knee (bone) & Tibia plat. Fx. & No & $0.2 \%($ AIS $2+)$ & No & $1.5 \%(\mathrm{AIS} 2+)$ & No \\
\hline L Knee (soft) & Ligament Rup. & LCL Rup. & $100 \%(\mathrm{AIS} 2+)$ & LCL $\underline{\text { ACL Rup. }}$ & $100 \%($ AIS $2+)$ & LCL Rup. \\
\hline L Knee (bone) & Tibia plat. Fx. & No & $0 \%(\mathrm{AIS} 2+)$ & No & $1.2 \%(\mathrm{AIS} 2+)$ & No \\
\hline R Ankle (soft) & Ligament Rup. & No & \multirow{2}{*}{$0 \%(\mathrm{AIS} 2+$ ) } & PTFL Rup. & \multirow{2}{*}{$8.9 \%$ (AIS2+) } & PTFL CFL Rup. \\
\hline R Ankle(bone) & Calcaneus/Talus Fx. & No & & No & & No \\
\hline L Ankle (soft) & Ligament Rup. & ATF Lig. Fx. & \multirow{2}{*}{$100 \%$ (AIS2+) } & CFL ATFL Rup. & \multirow{2}{*}{$99.2 \%(\mathrm{AIS} 2+)$} & CFL ATFL Rup. \\
\hline L Ankle (bone) & Calcaneus/Talus Fx. & No & & No & & No \\
\hline
\end{tabular}


Table 3.23 Predicted injury risk (morphed model1)

\begin{tabular}{|c|c|c|c|c|c|c|c|}
\hline \multicolumn{2}{|c|}{ PMHS Injury } & \multicolumn{3}{|c|}{ Function-based } & \multicolumn{3}{|c|}{ Strain-based } \\
\hline Region & Injury & Measurement & Value & Injury & Region & Max strain & Threshold \\
\hline Head & No injury & HIC & 1938 & $\underline{74.9 \%}$ & Skull & $0.5 \%$ & $1.5 \%$ \\
\hline Neck (soft) & $\begin{array}{l}\text { C6-C7 all } \\
\text { lig. rup.(AIS 2) }\end{array}$ & Not eva. & Not eva. & Not eva. & $\begin{array}{c}\text { CL } \\
\text { ISL LF PLL }\end{array}$ & $\begin{array}{c}261 \% \\
103 \% 75 \% \\
63 \%\end{array}$ & $\begin{array}{l}151 \% \\
50 \%\end{array}$ \\
\hline Neck (bone) & C6 SpProc.Fx.(AIS & Not eva. & Not eva. & Not eva. & C7 SpProc & $\underline{0.9 \%}$ & $1.5 \%$ \\
\hline Thorax R & No injury & Norm.ave.half.D & $6.6 \%$ & $1.1 \%$ & Rib 3 & $0.8 \%$ & $1.5 \%$ \\
\hline Thorax L & Rib 6 Fx. (AIS 1) & Norm.ave.half.D & $12.4 \%$ & $10.3 \%$ & Rib 7 rib 6 & $1.5 \% 1.5 \%$ & $1.5 \%$ \\
\hline Thorax M & No injury & Not eva. & Not eva. & Not eva. & Sternum & $0.3 \%$ & $1.5 \%$ \\
\hline Shoulder R & No injury & Clavicle axial & $10 \mathrm{~N}$ & Low risk & Clav. Scap. & $1.0 \% \underline{1.6 \%}$ & $1.5 \%$ \\
\hline Shoulder L & No injury & Clavicle axial & $20 \mathrm{~N}$ & Low risk & Clav. Scap. & $0.5 \% 0.4 \%$ & $1.5 \%$ \\
\hline Spine (soft) & $\begin{array}{l}\text { T11-12 PLL torn } \\
\text { (AIS 2) }\end{array}$ & Not eva & Not eva. & Not eva. & $\begin{array}{c}\text { CL } \\
\text { ISL ALL PLL }\end{array}$ & $\underline{69 \% 68 \%}$ & $\begin{array}{l}151 \% \\
50 \%\end{array}$ \\
\hline Spine (bone) & $\begin{array}{l}\text { T11 Endplate inf. } \\
\text { Fx.(AIS 2) }\end{array}$ & Not eva. & Not eva. & Not eva. & $\underline{\text { T12 }}$ & $\underline{0.5 \%}$ & $1.5 \%$ \\
\hline Pelvis R & Mult .Lat. Fx. (AIS & I atoral deflection & $35 \mathrm{~mm}$ & $790 \%$ & pubic bone & $\underline{0.8 \%}$ & $1 \%$ \\
\hline Pelvis L & No injury & & & & Acetabulum & $\underline{1.2 \%}$ & $1 \%$ \\
\hline R Thigh & No injury & Moment & $102 \mathrm{Nm}$ & $0 \%$ & Femur & $1.3 \%$ & $1.5 \%$ \\
\hline L Thigh & No injury & Moment & $123 \mathrm{Nm}$ & $0.1 \%$ & Femur & $0.5 \%$ & $1.5 \%$ \\
\hline R Leg & No injury & Moment & $160 \mathrm{Nm}$ & $1.5 \%$ & Tibia Fibula & $1.4 \% 1.0 \%$ & $1.5 \%$ \\
\hline L Leg & No injury & Moment & $96 \mathrm{Nm}$ & $0.1 \%$ & Tibia Fibula & $0.9 \% \underline{\mathbf{2 . 4} \%}$ & $1.5 \%$ \\
\hline R Knee (soft) & MCL ACL (AIS 2) & $\begin{array}{l}\text { Bending angle } \\
\text { Shear }\end{array}$ & $\begin{array}{c}44.5^{\circ} \\
12.3 \mathrm{~mm}\end{array}$ & $100 \%$ & $\begin{array}{l}\text { MCL } \\
\text { ACL }\end{array}$ & $\begin{array}{l}32 \% \\
24 \%\end{array}$ & $\begin{array}{l}28 \% \\
24 \%\end{array}$ \\
\hline $\begin{array}{l}\text { R Knee } \\
\text { (bone) }\end{array}$ & $\begin{array}{l}\text { Tib.Plat. Fx (Ant } \\
\text { Lat) (AIS 2) }\end{array}$ & Axial force & $1 \mathrm{KN}$ & $\underline{0.9 \%}$ & Tib.Plat. & $\leq 1.4 \%$ & $1.5 \%$ \\
\hline L Knee (soft) & No injury & $\begin{array}{l}\text { Bending angle } \\
\text { Shear }\end{array}$ & $\begin{array}{l}23.8^{\circ} \\
7.1 \mathrm{~mm}\end{array}$ & $100 \%$ & $\begin{array}{l}\text { LCL } \\
\text { ACL }\end{array}$ & $\begin{array}{l}31 \% \\
32 \%\end{array}$ & $\begin{array}{l}28 \% \\
24 \%\end{array}$ \\
\hline $\begin{array}{l}\text { L Knee } \\
\text { (bone) }\end{array}$ & $\begin{array}{l}\text { Tib.Plat Fx. (LCL) } \\
\text { (AIS 2) }\end{array}$ & Axial force & $1.5 \mathrm{KN}$ & $\underline{1.3 \%}$ & Tib.Plat. & $\leq 0.9 \%$ & $1.5 \%$ \\
\hline R Ankle & No injury & Bending angle & $9.5^{\circ}$ & $0.1 \%$ & CFL & $31 \%$ & $32 \%$ \\
\hline R Ankle & No injury & Axıal force & $1.6 \mathrm{~K}$ & & Calcaneus & $0.4 \%$ & $1.5 \%$ \\
\hline L Ankle & No injury & Bending angle & $62.2^{\circ}$ & $100 \%$ & CFL ATFL & $\underline{103 \% 73 \%}$ & $32 \%$ \\
\hline L Ankle & No injury & Axial force & $1.2 \mathrm{KN}$ & & Talus & $0.5 \%$ & $1.5 \%$ \\
\hline
\end{tabular}


Table 3.24 Predicted injury risk (baseline model1)

\begin{tabular}{|c|c|c|c|c|c|c|c|}
\hline \multicolumn{2}{|c|}{ PMHS Injury } & \multicolumn{3}{|c|}{ Function based } & \multicolumn{3}{|c|}{ Strain-based } \\
\hline Region & Injury & Measurement & Value & Injury & Region & Max strain & Threshold \\
\hline Head & No injury & HIC & 2210 & $\underline{80.8 \%}$ & Skull & $0.3 \%$ & $1.5 \%$ \\
\hline Neck (soft) & $\begin{array}{l}\text { C6-C7 all } \\
\text { lig. rup.(AIS 2) }\end{array}$ & Not eva. & $\begin{array}{l}\text { Not } \\
\text { eva. }\end{array}$ & Not eva. & $\begin{array}{l}\text { CL } \\
\text { ISL LF }\end{array}$ & $\begin{array}{c}201 \% \\
133 \% 80 \%\end{array}$ & $\begin{array}{c}151 \% \\
50 \%\end{array}$ \\
\hline Neck (bone) & C6 SpProc.Fx.(AIS 2) & Not eva. & $\begin{array}{l}\text { Not } \\
\text { eva. }\end{array}$ & Not eva. & C7 SpProc & $\underline{0.9 \%}$ & $1.5 \%$ \\
\hline Thorax R & No injury & Norm.ave.half.D & $12.8 \%$ & $11.8 \%$ & Rib 3 & $1.1 \%$ & $1.5 \%$ \\
\hline Thorax L & Rib 6 Fx. (AIS 1) & Norm.ave.half.D & $6.0 \%$ & $1.1 \%$ & Rib 8 & $1.2 \%$ & $1.5 \%$ \\
\hline Thorax M & No injury & Not eva. & Not & Not eva. & Sternum & $1.0 \%$ & $1.5 \%$ \\
\hline Shoulder R & No injury & Clavicle axial & $15 N$ & 0 & Clav. Scap. & $1.1 \% \underline{\mathbf{2 . 5} \%}$ & $1.5 \%$ \\
\hline Shoulder L & No injury & Clavicle axial & $8 \mathrm{~N}$ & 0 & Clav. Scap. & $0.7 \% 0.5 \%$ & $1.5 \%$ \\
\hline Spine (soft) & $\begin{array}{l}\text { T11-12 PLL torn } \\
\text { (AIS 2) }\end{array}$ & Not eva. & $\begin{array}{l}\text { Not } \\
\text { eva. }\end{array}$ & Not eva. & CL & $\underline{70 \%}$ & $\begin{array}{c}151 \% \\
50 \%\end{array}$ \\
\hline Spine (bone) & $\begin{array}{l}\text { T11 Endplate inf. Fx. } \\
\text { (AIS 2) }\end{array}$ & Not eva. & $\begin{array}{l}\text { Not } \\
\text { eva. }\end{array}$ & Not eva. & $\underline{\text { T3 }}$ & $\underline{0.8 \%}$ & $1.5 \%$ \\
\hline Pelvis R & Mult .Lat. Fx. (AIS 3) & Lateral deflection & $2.5 \mathrm{~mm}$ & $41 \%$ & $\begin{array}{l}\text { Acetabulum pubic } \\
\text { bone. }\end{array}$ & $2.5 \% 1.6 \%$ & $1 \%$ \\
\hline Pelvis L & No injury & & & & illium & $0.7 \%$ & $1 \%$ \\
\hline R Thigh & No injury & Moment & 205 & $1.7 \%$ & $\underline{\text { Femur }}$ & $\underline{1.5 \%}$ & $1.5 \%$ \\
\hline L Thigh & No injury & Moment & 239 & $4.3 \%$ & $\underline{\text { Femur }}$ & $2.2 \%$ & $1.5 \%$ \\
\hline R Leg & No injury & Moment & 181 & $3.1 \%$ & Tibia Fibula & $1.4 \% 1.1 \%$ & $1.5 \%$ \\
\hline L Leg & No injury & Moment & 205 & $6.2 \%$ & Tibia Fibula & $1.2 \% \underline{\mathbf{2 . 9} \%}$ & $1.5 \%$ \\
\hline $\begin{array}{l}\text { R Knee } \\
\text { (soft) }\end{array}$ & MCL ACL (AIS 2) & $\begin{array}{l}\text { Bending angle } \\
\text { Shear }\end{array}$ & $\begin{array}{c}39.3 \\
9.6 \mathrm{~mm}\end{array}$ & $100 \%$ & $\begin{array}{l}\text { MCL } \\
\text { ACL }\end{array}$ & $\begin{array}{l}30 \% \\
26 \%\end{array}$ & $\begin{array}{l}28 \% \\
24 \%\end{array}$ \\
\hline $\begin{array}{l}\text { R Knee } \\
\text { (bone) }\end{array}$ & $\begin{array}{l}\text { Tib.Plat. Fx (Ant Lat) } \\
\text { (AIS 2) }\end{array}$ & Axial force & 1.6 & $\underline{1.1 \%}$ & Tib.Plat. & $\leq 1.4 \%$ & $1.5 \%$ \\
\hline $\begin{array}{l}\text { L Knee } \\
\text { (soft) }\end{array}$ & No injury & $\begin{array}{l}\text { Bending angle } \\
\text { Shear }\end{array}$ & $\begin{array}{l}28.3 \\
8 \mathrm{~mm}\end{array}$ & $100 \%$ & LCL & $29 \%$ & $\begin{array}{l}28 \% \\
24 \%\end{array}$ \\
\hline L Knee & Tib.Plat Fx. (LCL) (AIS & Axial force & 1.8 & $\underline{1.3 \%}$ & Tib.Plat. & $\leq 0.9 \%$ & $1.5 \%$ \\
\hline R Ankle & No injury & Bending angle & 5.5 & $0 \%$ & CFL PTFL & $40 \% 37 \%$ & $32 \%$ \\
\hline R Ankle & No injury & Axial force & 1.7 & & Calcaneus & $0.3 \%$ & $1.5 \%$ \\
\hline $\begin{array}{l}\text { L Ankle } \\
\text { (soft) }\end{array}$ & No injury & $\begin{array}{l}\text { Bending angle } \\
\text { Axial force }\end{array}$ & $\begin{array}{c}67.8 \\
2.6\end{array}$ & $\underline{99.8 \%}$ & CFL ATFL PTFL & $\frac{90 \% 77 \%}{\underline{50 \%}}$ & $32 \%$ \\
\hline L Ankle & No injury & & & & Talus & $0.4 \%$ & $1.5 \%$ \\
\hline
\end{tabular}


Table 3.25 Predicted injury risk (morphed model 2)

\begin{tabular}{|c|c|c|c|c|c|c|c|}
\hline \multicolumn{2}{|c|}{ PMHS Injury } & \multicolumn{3}{|c|}{ Function based } & \multicolumn{3}{|c|}{ Strain-based } \\
\hline Region & Injury & Measurement & value & Injury & Component & Max strain & Threshold \\
\hline Head & No injury & HIC & 1136 & $\underline{53.4 \%}$ & Skull & $0.3 \%$ & $1.5 \%$ \\
\hline Neck (soft) & No injury & Not eva. & Not eva. & Not eva. & ISL $\frac{\text { CL }}{\text { PLL LF }}$ & $\frac{688 \%}{557 \% 184 \%}$ & $\begin{array}{l}151 \% \\
50 \%\end{array}$ \\
\hline Neck (bone) & No injury & Not eva. & Not eva. & Not eva. & C7 SpProc & $\underline{2.5 \%}$ & $1.5 \%$ \\
\hline Thorax R & rib 2-6 Fx. (AIS 4) & Norm.ave.half.D & $22 \%$ & $81 \%$ & $\operatorname{rib}(2,3,4)$ & 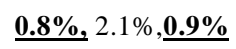 & $1.5 \%$ \\
\hline Thorax L & rib 4-7 Fx. (AIS 4) & Norm.ave.half.D & $18 \%$ & $47.8 \%$ & rib 3 & $\underline{1.5 \%}$ & $1.5 \%$ \\
\hline Thorax M & Sternal Fx. (AIS 2) & Not eva. & Not eva. & Not eva. & $\underline{\text { Sternum }}$ & $\underline{1.1 \%}$ & $1.5 \%$ \\
\hline Shoulder R & No injury & Clavicle axial Force & $40 \mathrm{~N}$ & Low risk & Clav. Scap. & $\underline{\mathbf{1 . 6} \%} 1 \%$ & $1.5 \%$ \\
\hline Shoulder L & Scap. Fx. (AIS 2) & Clavicle axial Force & $30 \mathrm{~N}$ & Low risk & Clav. $\underline{\text { Scap. }}$ & $1.1 \% 4.2 \%$ & $1.5 \%$ \\
\hline Spine (soft) & $\begin{array}{l}\text { T11-12 all lig. rup. } \\
\text { (AIS 2) }\end{array}$ & Not eva. & Not eva. & Not eva. & $\frac{\mathbf{C L}}{\mathrm{ALL}}$ & $\frac{\mathbf{9 0} \%}{51 \%}$ & $\begin{array}{c}151 \% \\
50 \%\end{array}$ \\
\hline Spine (bone) & $\begin{array}{l}\text { T12 Sup Endplate } \\
\text { Fx (AIS 2). }\end{array}$ & Not eva. & Not eva. & Not eva. & $\underline{\mathbf{T} 3}$ & $\underline{1.5 \%}$ & $1.5 \%$ \\
\hline Abdomen & No injury & Not eva. & Not eva. & Not eva. & $\underline{\text { Liver }}$ & $\underline{77 \% 38 \%}$ & $30 \%$ \\
\hline Pelvis R & $\begin{array}{l}\text { Mult.Lat.Comp.Fx. } \\
\text { (AIS 3) }\end{array}$ & Lateral deflection & $1.6 \mathrm{~mm}$ & $\underline{11.2 \%}$ & Acetabulum & $1.3 \%$ & $1 \%$ \\
\hline Pelvis L & No injury & & & & Ilium & $\underline{0.6 \%}$ & $1 \%$ \\
\hline R Thigh & No injury & Moment & $454 \mathrm{Nm}$ & $\underline{91 \%}$ & Femur & $1.3 \%$ & $1.5 \%$ \\
\hline L Thigh & No injury & Moment & $249 \mathrm{Nm}$ & $5.5 \%$ & Femur & $0.6 \%$ & $1.5 \%$ \\
\hline R Leg & No injury & Moment & $144 \mathrm{Nm}$ & $6.8 \%$ & Tibia Fibula & $1.1 \% 1.0 \%$ & $1.5 \%$ \\
\hline L Leg & No injury & Moment & $116 \mathrm{Nm}$ & $0.3 \%$ & Tibia Fibula & $0.7 \% \underline{\mathbf{2 . 0} \%}$ & $1.5 \%$ \\
\hline R Knee (soft) & No injury & $\begin{array}{l}\text { Bending angle } \\
\text { Shear }\end{array}$ & $\begin{array}{c}33^{\circ} \\
11.3 \mathrm{~mm}\end{array}$ & $\underline{100 \%}$ & $\frac{\text { MCL }}{\text { ACL }}$ & $\frac{31 \%}{29 \%}$ & $\begin{array}{l}28 \% \\
24 \%\end{array}$ \\
\hline R Knee & No injury & Axial force & $2.8 \mathrm{KN}$ & $0.2 \%$ & Tib.Plat. & $<1.1 \%$ & $1.5 \%$ \\
\hline L Knee (soft) & LCL rup. (AIS 2) & $\begin{array}{l}\text { Bending angle } \\
\text { Shear }\end{array}$ & $\begin{array}{c}37.8^{\circ} \\
6.7 \mathrm{~mm}\end{array}$ & $100 \%$ & $\begin{array}{l}\text { LCL } \\
\text { ACL }\end{array}$ & $\begin{array}{l}36 \% \\
\mathbf{3 6 \%}\end{array}$ & $\begin{array}{l}28 \% \\
24 \%\end{array}$ \\
\hline L Knee & No injury & Axial force & $1.2 \mathrm{KN}$ & $0 \%$ & Tib.Plat. & $<0.7 \%$ & $1.5 \%$ \\
\hline R Ankle (soft) & No injury & Bending angle & $9^{\circ}$ & $0 \%$ & PTFL & $\underline{44 \%}$ & $32 \%$ \\
\hline R Ankle & No injury & Axial force & $3.3 \mathrm{KN}$ & $0 \%$ & Calcaneus & $0.3 \%$ & $1.5 \%$ \\
\hline L ankle (soft) & $\begin{array}{l}\text { ATF Lig. Fx. } \\
\text { (AIS 2) }\end{array}$ & $\begin{array}{l}\text { Bending angle } \\
\text { Axial force }\end{array}$ & $\begin{array}{c}61^{\circ} \\
1.1 \mathrm{KN}\end{array}$ & $100 \%$ & $\underline{\text { CFL ATFL }}$ & $\underline{73 \%} 66 \%$ & $32 \%$ \\
\hline L Ankle & No injury & & & & Talus & $0.4 \%$ & $1.5 \%$ \\
\hline
\end{tabular}


Table 3.26 Predicted injury risk (baseline model 2)

\begin{tabular}{|c|c|c|c|c|c|c|c|}
\hline \multicolumn{2}{|c|}{ PMHS Injury } & \multicolumn{3}{|c|}{ Function based } & \multicolumn{3}{|c|}{ Strain-based } \\
\hline Region & Injury & Measurement & value & Injury & Component & Max strain & Threshold \\
\hline Head & No injury & HIC & 4530 & $\underline{95.7 \%}$ & Skull & $1.7 \%$ & $1.5 \%$ \\
\hline Neck (soft) & No injury & Not eva. & $\begin{array}{l}\text { Not } \\
\text { eva. }\end{array}$ & Not eva. & $\underline{\text { ISL LF }}$ & $\begin{array}{c}\underline{210 \%} \\
\underline{237 \% 108 \%} \\
\end{array}$ & $\begin{array}{l}151 \% \\
50 \%\end{array}$ \\
\hline Neck (bone) & No injury & Not eva. & Not & Not eva. & $\underline{\mathrm{C} 1}$ & $1.6 \%$ & $1.5 \%$ \\
\hline Thorax R & rib 2-6 Fx. (AIS 4) & Norm.ave.half.D & $11.5 \%$ & $7.5 \%$ & rib 3 & $1.1 \%$ & $1.5 \%$ \\
\hline Thorax L & rib 4-7 Fx. (AIS 4) & Norm.ave.half.D & $5.5 \%$ & $0.1 \%$ & $\underline{\text { rib } 8}$ & $1.1 \%$ & $1.5 \%$ \\
\hline Thorax M & Sternal Fx. (AIS 2) & Not eva. & $\begin{array}{l}\text { Not } \\
\text { eva. }\end{array}$ & Not eva. & $\underline{\text { Sternum }}$ & $\underline{1 \%}$ & $1.5 \%$ \\
\hline Shoulder R & No injury & Clavicle axial & 15 & 0 & Clav. Scap. & $0.8 \% 2.3 \%$ & $1.5 \%$ \\
\hline Shoulder L & Scap. Fx. (AIS 2) & Clavicle axial & 9 & 0 & Clav. $\underline{\text { Scap. }}$ & $0.6 \% 0.6 \%$ & $1.5 \%$ \\
\hline Spine (soft) & $\begin{array}{l}\text { T11-12 all lig. rup. } \\
\text { (AIS 2) }\end{array}$ & Not eva. & $\begin{array}{l}\text { Not } \\
\text { eva. }\end{array}$ & Not eva. & $\frac{\mathbf{C L}}{\mathrm{ALL}}$ & $\underline{90 \%}$ & $\begin{array}{l}151 \% \\
50 \%\end{array}$ \\
\hline Spine (bone) & $\begin{array}{l}\text { T12 Sup Endplate } \\
\text { Fx (AIS 2). }\end{array}$ & Not eva. & $\begin{array}{l}\text { Not } \\
\text { eva. }\end{array}$ & Not eva. & $\underline{\mathrm{T} 3}$ & $\underline{0.8 \%}$ & $1.5 \%$ \\
\hline Pelvis R & $\begin{array}{l}\text { Mult.Lat.Comp.Fx. } \\
\text { (AIS 3) }\end{array}$ & Lateral deflection & $2.3 \mathrm{~mm}$ & $\underline{32.7 \%}$ & $\begin{array}{l}\text { Acetabulum pubic } \\
\text { bone }\end{array}$ & $2.2 \% 1.8 \%$ & $1 \%$ \\
\hline Pelvis L & No injury & & & & Ilium & $0.8 \%$ & $1 \%$ \\
\hline R Thigh & No injury & Moment & 237 & $\underline{4.1 \%}$ & $\underline{\text { Femur }}$ & $\underline{1.5 \%}$ & $1.5 \%$ \\
\hline L Thigh & No injury & Moment & 224 & $2.9 \%$ & $\underline{\text { Femur }}$ & $\underline{2.0 \%}$ & $1.5 \%$ \\
\hline R Leg & No injury & Moment & 181 & $3.1 \%$ & Tibia Fibula & $1.3 \% 1.0 \%$ & $1.5 \%$ \\
\hline L Leg & No injury & Moment & 204 & $6 \%$ & Tibia Fibula & $1.2 \%$ & $1.5 \%$ \\
\hline R Knee (soft) & No injury & $\begin{array}{c}\text { Bending angle } \\
\text { Shear } \\
\text { displacement }\end{array}$ & $\begin{array}{c}41.6 \\
14 \mathrm{~mm}\end{array}$ & $\underline{100 \%}$ & $\frac{\text { MCL }}{\underline{\text { ACL }}}$ & $\frac{28 \%}{\underline{25 \%}}$ & $\begin{array}{l}28 \% \\
24 \%\end{array}$ \\
\hline R Knee & No injury & Axial force & 2.0 & $1.5 \%$ & Tib.Plat. & $<1.1 \%$ & $1.5 \%$ \\
\hline L Knee (soft) & No injury & $\begin{array}{l}\text { Bending angle } \\
\text { Shear }\end{array}$ & $\begin{array}{c}28.3 \\
8 \mathrm{~mm}\end{array}$ & $100 \%$ & LCL & $29 \%$ & $\begin{array}{l}28 \% \\
24 \%\end{array}$ \\
\hline L Knee & LCL rup. (AIS 2) & Axial force & 1.7 & $1.2 \%$ & Tib.Plat. & $<0.7 \%$ & $1.5 \%$ \\
\hline R Ankle & No injury & Bending angle & 27.5 & $89 \%$ & PTFL CFL & $34 \% 32 \%$ & $32 \%$ \\
\hline R Ankle & No injury & Axial force & & 0.070 & Calcaneus & $0.3 \%$ & $1.5 \%$ \\
\hline L ankle (soft) & No injury & Bending angle & 63.1 & $992 \%$ & $\underline{\text { CFL ATFL }}$ & $\underline{\mathbf{7 6 \%}} 73 \%$ & $32 \%$ \\
\hline L Ankle & ATF Lig. Fx. & Nat iorce & & & Talus & $0.4 \%$ & $1.5 \%$ \\
\hline
\end{tabular}




\section{$\underline{\text { Chapter } 4}$ Evaluating Pedestrian Sub-system Test Procedure against}

\section{Full-scale Vehicle-Pedestrian Impact}

In chapter 3, the pedestrian FE human model was comprehensively evaluated and this provided the necessary tools for the main objective of this dissertation, which is to investigate the relationship between the impactor response in current pedestrian sub-system test and the pedestrian head response in full-scale pedestrian impact. In this chapter, a direct comparison will be conducted between the impactor response in the current Euro-NCAP test procedure and pedestrian head response in the full-scale vehicle-pedestrian impact. Then a further study conducted that will test the hypothesis that vehicle stiffness design variations have different influences on the impactor response in the sub-system test and head response in pedestrian fullscale impact.

\subsection{Introduction}

Chapter 2 introduced the serious problem of pedestrian accidents and the importance of head injury in pedestrian fatality. To combat this problem, the pedestrian sub-system test procedure was proposed and used in practice for decades. Although the debate and critique of the limitations of the simplified sub-system test procedure have lasted for decades (Kerrigan et al. 2008; Hardy et al. 2007), it is still the only testing method used to evaluate vehicle aggressiveness due to the important advantages of being repeatable, easy to conduct, and low cost. As a result, the industry is encouraged to optimize their vehicle design towards this 
component test procedure instead of the full-scale pedestrian impact. This poses a big question regarding how accurately the sub-system test procedure can represent the full-scale impact.

To date, no comprehensive study has been done to answer this question, partly due to the limited power of the methods and tools that currently exist. The cadaver test cannot serve this purpose because they are not repeatable, and each subject has different intrinsic conditions, making the parametric study not feasible. The dummy test cannot do this either, because testing the various vehicle designs involves many tests at a huge cost, and there is limited availability on anthropometry of the dummy. Finally, the multi-body models cannot accurately model the vehicle deformation, head-neck interaction, and the head impact geometry, and thus are not the most appropriate tools.

Recently, the development of the FE human model and vehicle model provided tools with high accuracy and enough complexity to answer this question. One of the PFEM, the THUMS pedestrian model, has been evaluated in many studies at the component level and fullscale level. In chapter 3, this model was evaluated on segmental velocity, acceleration, and whole-body injury, using geometrically personalized pedestrian model to consider the anthropometry dependence, and the model was deemed biofidelic in evaluation at both component and full-scale level.

Untaroiu et al. (2007) developed and validated an adult headform impactor FE model. The geometry was obtained from the drawing of the physical headform for the adult head test procedure while the skin material model was modeled as viscoelastic material with parameters identified by FE optimization to match quasi-static and dynamic test data reported in the literature (Matsui and Tanahashi 2004). Overall, the model showed that the geometrical and 
inertial characteristics of the headform FE model developed in this study satisfy compliance and certification regulations of ISO and IHRA, which is adopted by the current Euro-NCAP.

A previous accident analysis (Kawabe et al. 2012), full-scale PMHS test (Kerrigan et al. 2005, 2008; Subit et al. 2008; Paas et al. 2015), full-scale dummy test (Okamoto et al. 2003), FE model simulation (Watanabe et al. 2012; Han et al. 2012), and multi-body model simulation (Simms and Wood 2006; Elliott et al. 2012) all indicated that pedestrian response depends on anthropometry, posture, and initial location. Therefore, the impact scenario should be considered to fully investigate the pedestrian response.

The objective of this chapter is to directly compare the impactor response in the current Euro-NCAP test procedure to the pedestrian head response in the full-scale vehicle-pedestrian impact in diverse impact scenarios, and then test the hypothesis that vehicle stiffness design variations have different influences on the impactor response in the sub-system test and head response in pedestrian full-scale impact.

\subsection{Method and Material}

\subsubsection{Model Setup}

THUMS PFEM evaluated in chapter 3 was integrated with the vehicle FE model to simulate the full-scale pedestrian impact. The headform developed and validated against ISO and IHRA corridor by Untaroiu et al. (2007) was used to perform the current Euro-NCAP sub-system test.

Previous studies showed that 66-82\% of pedestrians were hit by passenger cars (Hardy et al. 2007), and the current test procedure was originally developed for "classical" (sedan type) passenger cars (Global technical regulation No. 9. 2008). Therefore, the mid-size sedan FE 
model used in the previous chapter for the THUMS biofidelity evaluation is used to represent a mid-size sedan type car. The geometry of the front-end of this vehicle model is verified to be entirely in the geometry corridor of 34 representative sedan type car models from 2008-2011 (Nie et al. 2016).

The head impact locations in all the full-scale impacts were recorded, and then were used as the target impact location in the sub-system impact simulation. The impactor test followed the current Euro-NCAP test procedure, with the headform mass of $4.5 \mathrm{~kg}$, speed of $40 \mathrm{~km} / \mathrm{h}$, and angle of $65^{\circ}$ with horizontal towards hood surface and windshield.

\section{Impact scenarios for full-scale simulation}

The $40 \mathrm{~km} / \mathrm{h}$ vehicle impact speed was chosen as the basis of the regulatory speed, and most PMHS and simulation studies have focused on this vehicle speed in previous studies. Indepth accident investigations in 5 countries (Chen et al. 2009; Mizuno 2005) have shown that more than $60 \%$ of pedestrian accidents were under $40 \mathrm{~km} / \mathrm{h}$, and the peak of the frequency of vehicle impact speed was around $40 \mathrm{~km} / \mathrm{h}$. Also, the pedestrian AIS 2+ injury risk was higher than $70 \%$ at $40 \mathrm{~km} / \mathrm{h}$. For all these reasons, $40 \mathrm{~km} / \mathrm{h}$ is chosen as the vehicle impact speed in the following simulations.

As the baseline model, referred to as "S", the pedestrian model was positioned in front of the vehicle at the vehicle centerline, facing laterally, in a standing posture with arms free down and legs aligned. Two walking postures (without walking speed) most distinct from standing during the whole gait cycle, referred to as "SF" or "RF" and "SB" or "LF", were from gait 0\% (struck leg forward) and 50\% (struck leg backward) (Untaroiu et al. 2009). The pedestrian model was positioned using the angles of hip, knee, shoulder and elbow developed by Untaroiu et al. (2009) based on a continuous sequence of the pedestrian gait. Previous studies found the SB 
posture generally causes positive torso rotation leading to anterior head impact and lower head impact speed, while the SF posture generally causes negative torso rotation leading to posterior head impact and higher head impact speed, and the S posture generally causes less torso rotation (Chen et al 2015; Elliott et al 2012). Therefore, 3 postures were used in this study to attempt to generate positive torso rotation, negative torso rotation, and no or few torso rotation, allowing for all types of pedestrian torso rotations to be covered.

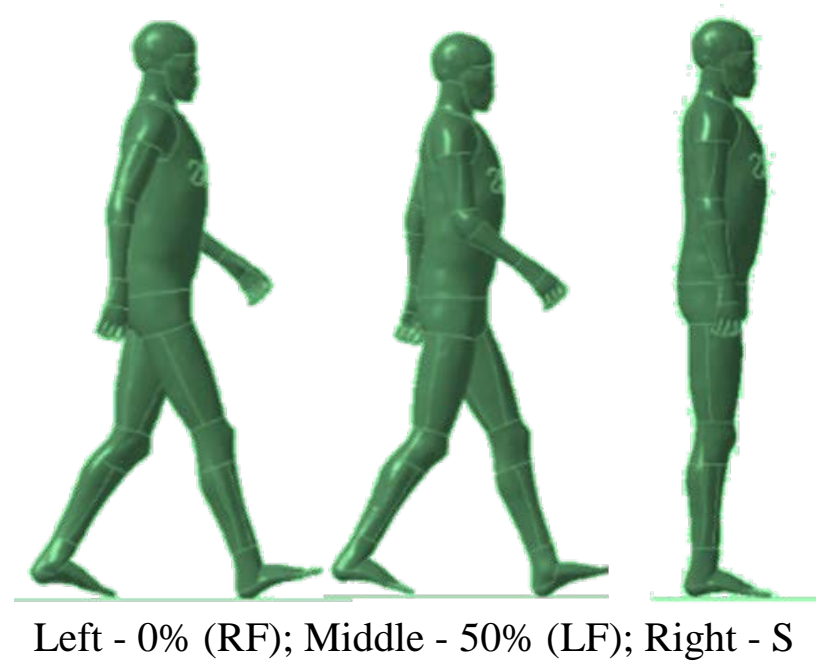

Figure 4.1 Impact scenarios of full-scale pedestrian impact - pedestrian postures

A pedestrian anthropometry series was created using global scaling, including $15^{\text {th }}$ percentile male, $50^{\text {th }}$ percentile male, and $85^{\text {th }}$ percentile male (Gordon et al 2012), which covers $50^{\text {th }}$ and $50^{\text {th }} \pm 1 \mathrm{SD}$ (standard deviation) of pedestrian global anthropometry. In the global scaling method, the pedestrian height was scaled first to the target height, and the width and depth are then uniformly scaled to the target mass $\left(\lambda_{z}=h / h_{0}, \lambda_{x}=\lambda_{y}=\sqrt{\frac{h_{0} * m}{h * m_{0}}}\right)$, while $h_{0}$ and $m_{0}$ are original height and mass of THUMS model. The anthropometry data of those three models are listed in Table 4.1. 


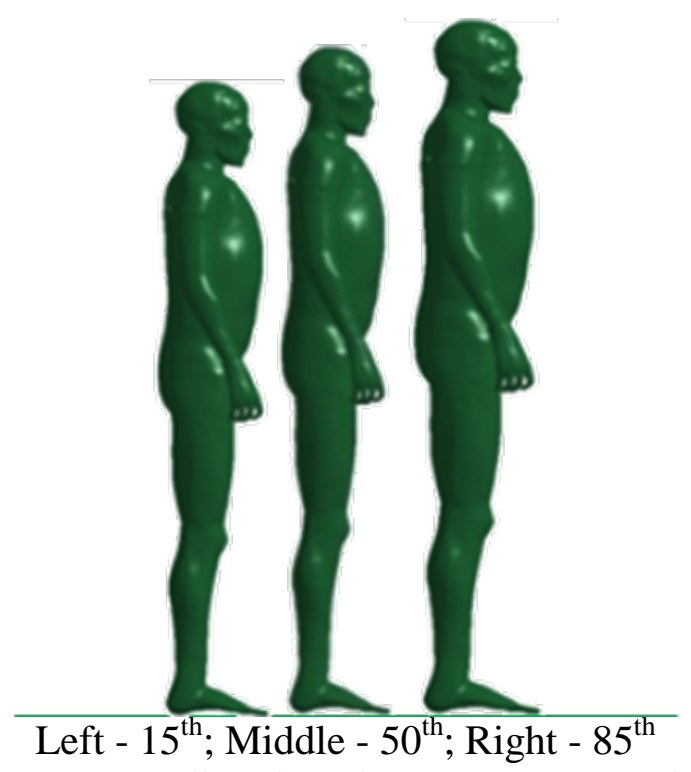

Figure 4.2 Impact scenarios of full-scale pedestrian impact - pedestrian anthropometry

Table 4.1 Anthropometry of the pedestrian series

\begin{tabular}{lccccccc}
\hline & Height $(\mathrm{mm})$ & Weight $(\mathrm{kg})$ & BMI $\left(\mathrm{kg} / \mathrm{m}^{2}\right)$ & Head mass $(\mathrm{kg})$ & $\lambda_{\mathrm{x}}$ & $\lambda_{\mathrm{y}}$ & $\lambda_{\mathrm{z}}$ \\
\hline $15^{\text {th }}$ & 1686 & 67.2 & 23.6 & 3.768 & 0.981 & 0.981 & 0.951 \\
$50^{\text {th }}$ & 1755 & 77.7 & 25.2 & 4.278 & 1.034 & 1.034 & 0.990 \\
$85^{\text {th }}$ & 1826 & 90.0 & 27.0 & 5.081 & 1.091 & 1.091 & 1.030 \\
\hline
\end{tabular}

Two impact locations were covered, including the center, and the corner which is 400 $\mathrm{mm}$ from the center along the vehicle transverse direction. Three initial orientations were simulated including the pure lateral direction $\left(0^{\circ}\right)$, facing vehicle $\left(-90^{\circ}\right)$, and facing away from the vehicle $\left(90^{\circ}\right)$.

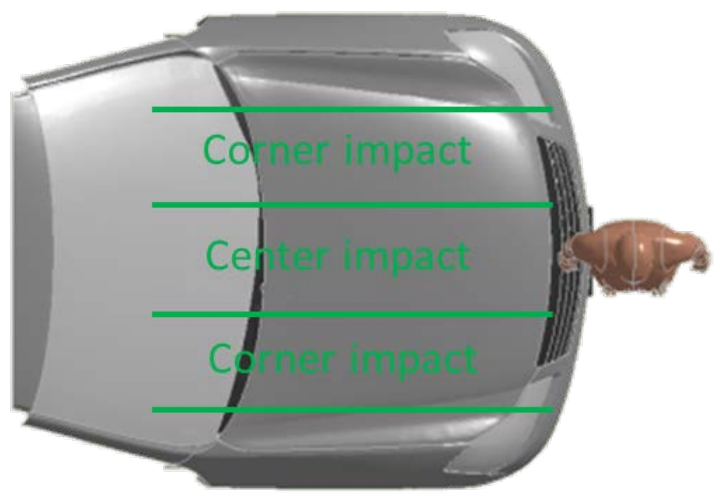


Figure 4.3 Impact scenarios of full-scale pedestrian impact - Pedestrian locations

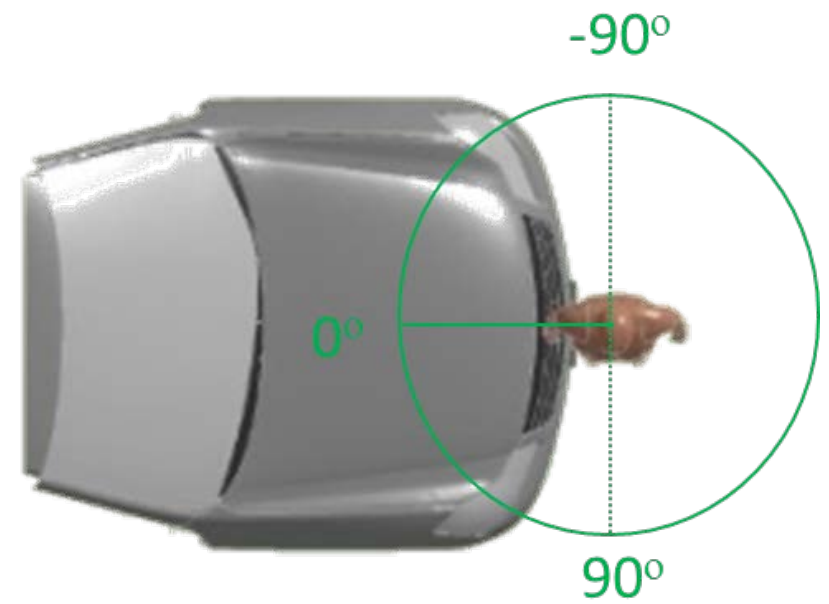

Figure 4.4 Impact scenarios of full-scale pedestrian impact - Pedestrian orientations

Since these impact scenarios are not equally distributed in real accidents, the probability distribution from the literatures (Chidester et al. 2001; Neal-Strugess et al. 2007; Soni et al. 2013; Hamdane et al. 2014) were indicated in Figure 4.4 as the reference to estimate the relative frequency of them. 


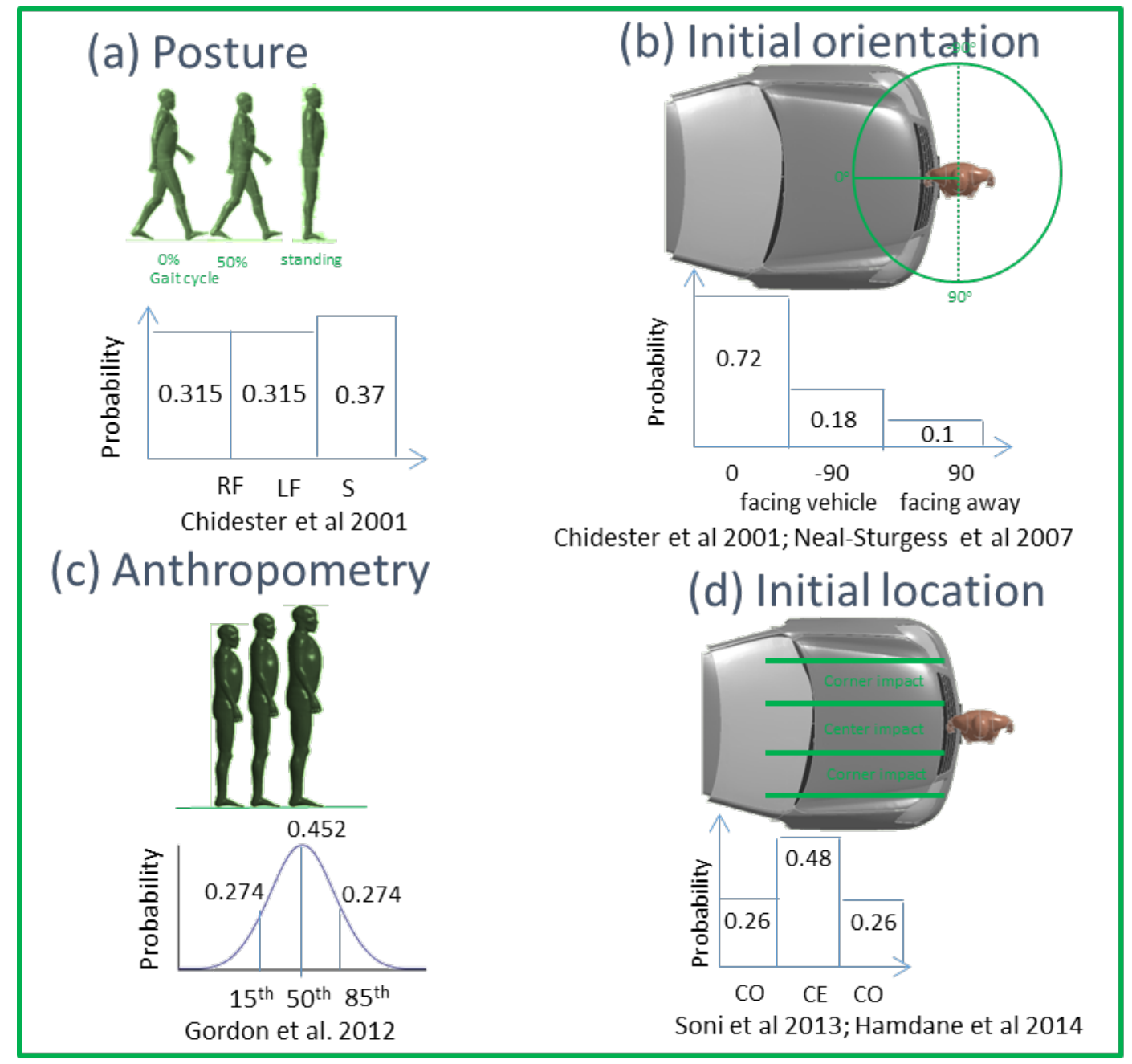

Figure 4.5 Impact scenarios of full-scale pedestrian impact and their frequencies

\section{Vehicle stiffness design variation}

The vehicle stiffness on the head impact area was varied to perform both the component impact experiment and the full-scale impact experiment. The vehicle design variations are listed in Table 4.2.

Two of three possible impact configurations were considered. Configuration 1 is the head impacting with the windshield or frame, and in this configuration there is no pre-deformation by the torso on the head impact area. Configuration 2 is the head impacting with the hood surface, 
and in this configuration the hood is already pre-deformed by the torso before the head impact. Configuration 3, which is the head impacting both the hood and windshield was not considered for vehicle design variation.

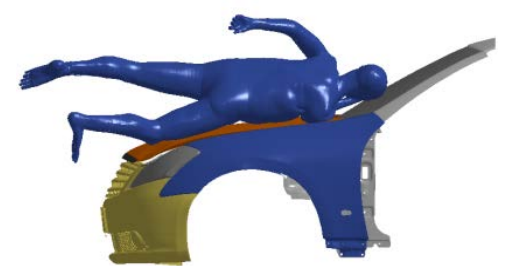

Impact configuration $1(n=24)$

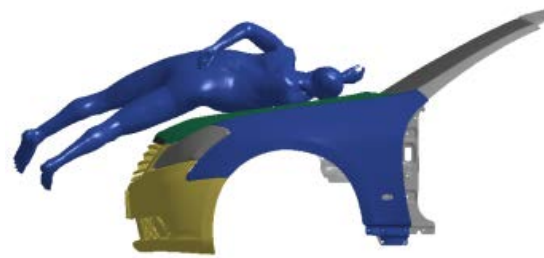

Impact configuration $2(n=11)$

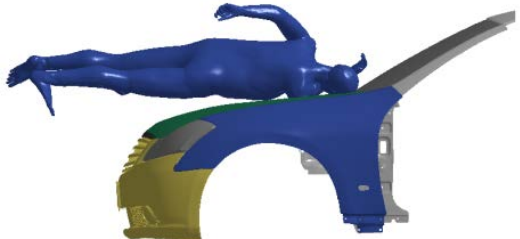

Impact configuration $3(n=19)$

Figure 4.6 Three impact configurations

The vehicle stiffness was varied by modifying the material property and thickness in the reasonable range. For impact configuration 1, on the 3 layer windshield, the Young's modulus of plastic interlayer and the yield stress of outer glass layer were slightly decreased relative to the baseline vehicle model (Munsch et al. 2009).

For impact configuration 2, on the hood, 2 material types were used. The baseline model uses the steel material on the hood outer and inner panel, while the varied vehicle designs use aluminum on the outer panel, or inner panel (Kerkeling et al. 2005), or both. Three hood thickness levels were used. The baseline model uses $0.97 \mathrm{~mm}$ and $0.87 \mathrm{~mm}$ on the hood outer and inner panel, while the varied vehicle designs use $0.5 \mathrm{~mm}$ or $2 \mathrm{~mm}$ (Liu et al. 2009) on outer panel, or inner panel, or both. The hood mountings of hinge support were varied by modifying the material and thickness of hinges on both sides to strengthen or weaken the boundary support (Kerkeling et al. 2005). The baseline vehicle model uses the steel material with $3.3 \mathrm{~mm}$ thickness, while the weakening design uses aluminum material and 2mm thickness, and the strengthening design uses the Tungsten material and $6 \mathrm{~mm}$ thickness. 
Table 4.2 Vehicle stiffness design variation

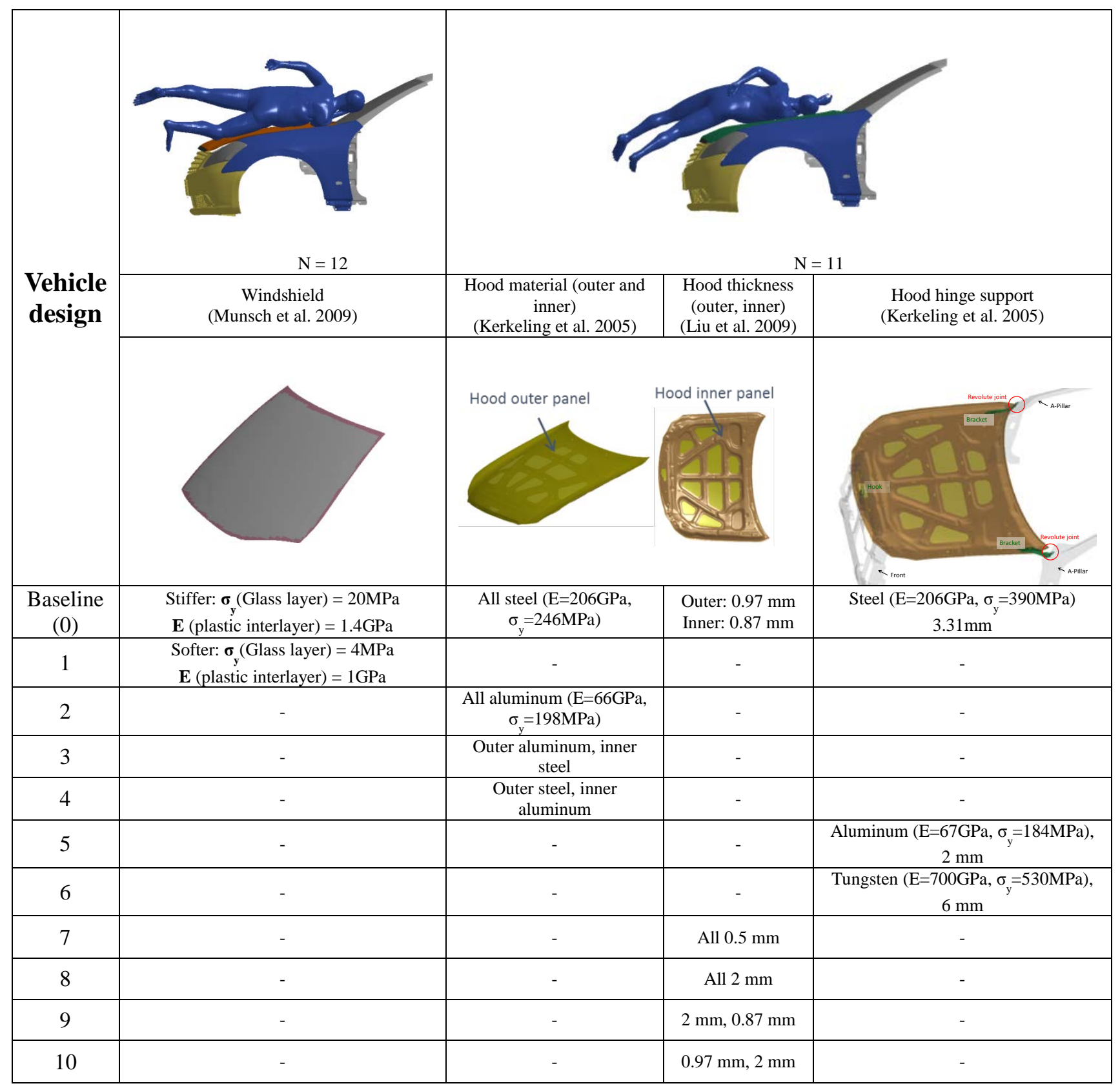




\section{$\underline{\text { Simulation matrix }}$}

In total 129 full-scale impacts and 110 sub-system tests were performed.

At the first step, as shown in Figure 4.4 , to cover 3 postures, 3 orientations, 3 anthropometries, and 2 locations, 54 full-scale experiments were performed.

At the second step, accordingly, 35 component experiments (19 cases with impact configuration 3 were excluded here) were performed at the same impact location with the fullscale experiments to simulate the current Euro-NCAP pedestrian head sub-system impactor tests.

At the last step, in 25 impact scenarios that caused impact configuration 1, 12 of them that did not impact with the windshield frame were varied with windshield stiffness. All the 11 impact scenarios that caused impact configuration 2 were varied to strengthen (11 simulation) or weaken (11 simulation) hinge support, aluminum material on both hood outer and hood inner (11 simulation), $0.5 \mathrm{~mm}$ thickness on both hood outer and hood inner (11 simulation), and 2mm thickness on both hood outer and hood inner (11 simulation). Finally, 2 baseline models with higher frequency of impact scenarios, RF15 and LF15, were varied to aluminum material on hood outer or hood inner (4 simulation), and $2 \mathrm{~mm}$ thickness on hood outer or hood inner (4 simulation). This step will require 75 full-scale impact experiments and accordingly 75 component impact experiments.

\subsubsection{Head Response Comparison}

The time history of head acceleration and head impact force in full-scale impact and subsystem test are output and compared. The force and velocity are digitally filtered with a CFC180 SAE channel class filter, while the acceleration is filtered with a CFC1000 SAE channel class filter. 
The head impact conditions including head impact location (wrap around distance WAD) head impact speed, angle, and effective mass were calculated for the full-scale impact. The head impact speed and angle are calculated by the following formulas, where $V_{y}$ is the relative head horizontal velocity against the vehicle and $V_{z}$ is the relative head vertical velocity against the vehicle (Chen et al. 2009). The head impact locations were classified by WAD into three areas as Figure 4.7.

$$
\begin{gathered}
V=\left(V_{y}^{2}+V_{z}^{2}\right)^{1 / 2} \\
\alpha=\arctan \left(V_{z} / V_{y}\right)
\end{gathered}
$$

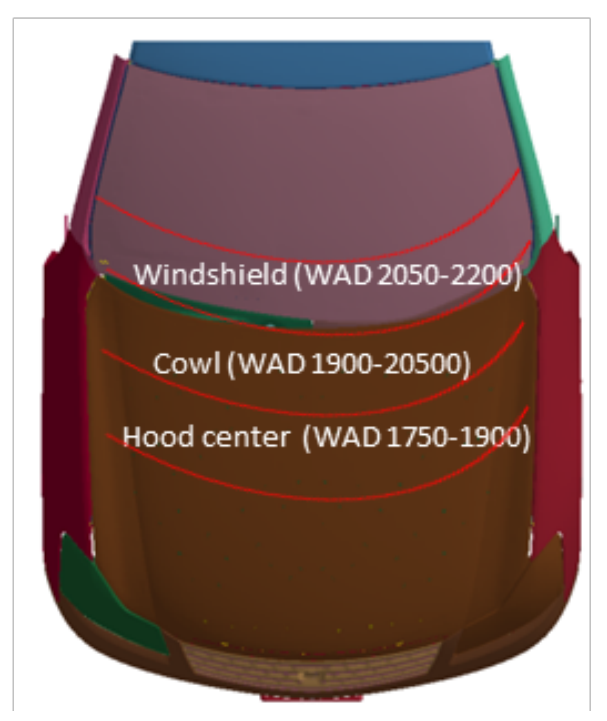

Figure 4.7 Head impact locations by WAD

The effective mass is calculated by effective average force divided by the effective average acceleration, as shown in equation 4.3 , where the starting time $t_{1}$ is when head contact force becomes non-zero, and the end time $t_{2}$ is when head normal velocity relative to the vehicle contact surface becomes zero. 


$$
m=\frac{\int_{t 1}^{t 2} F d t}{\Delta v}=\frac{\int_{t 1}^{t 2} F d t}{\int_{t 1}^{t 2} a d t}
$$

The HIC is used as the main indicator for head response because it is the only measurement for the current test procedure. The BrIC is output and compared only in the fullscale impacts because the current test procedure is a linear impact test as the initial input condition. The peak impact forces are output only as reference because it doesn't include the time duration and thus was deemed not good enough to indicate injury risk.

The paired-T test was performed to examine the significance on the difference of head response or impactor response between the baseline vehicle design and varied vehicle design, and the $\mathrm{p}=0.05$ was set as the threshold for significance. To estimate the effect of vehicle stiffness designs, the ratio $(\mathrm{k})$ of the mean head response and impactor responses from varied vehicle stiffness designs were calculated and indicated by the slope of the linear line in the graphs.

\subsection{Results}

The pedestrian head response in the full-scale vehicle-pedestrian impacts in diverse impact scenarios and the impactor response in the current Euro-NCAP test procedure were compared, and the effect of vehicle designs on head response in both sub-system tests and pedestrian full-scale impacts were observed, and the results are listed and discussed here.

\subsubsection{Pedestrian Head Impact Conditions in Full-scale Impact}

The head impact conditions were highly dependent on the pedestrian initial impact direction. The head impact speeds in the 36 non-lateral impacts $(16.5 \pm 0.6 \mathrm{~m} / \mathrm{s})$ were 
significantly higher than in the 18 lateral impact scenarios $(10.7 \pm 2.1 \mathrm{~m} / \mathrm{s})$, and the head impact angle in the non-lateral impacts $(64.3 \pm 9.6 \mathrm{~m} / \mathrm{s})$ were also higher than in the lateral impact scenarios $(51.6 \pm 4.5 \mathrm{~m} / \mathrm{s})$. The head effective mass was also higher in the 19 non-lateral impacts $(1.0 \pm 0.1$ times of original head mass) than in the 15 lateral impact scenarios $(0.8 \pm 0.1 \mathrm{~m} / \mathrm{s}$ times of original head mass).

The head impact conditions were also location-dependent. The area with lower WAD 1750-1900 obtained lower head impact speed but higher impact angle. The head effective mass increases with WAD, but the ratios of effective mass relative to head original mass were not very different for the three areas (Table 4.3).

Table 4.3 Location dependence of head impact conditions

\begin{tabular}{cccccc}
\hline & $\begin{array}{c}\text { Head impact } \\
\text { timing }(\mathrm{ms})\end{array}$ & $\begin{array}{c}\text { Head impact speed } \\
(\mathrm{m} / \mathrm{s})\end{array}$ & $\begin{array}{c}\text { Head impact } \\
\text { angle }\left(^{\circ}\right)\end{array}$ & $\begin{array}{c}\text { Head effective } \\
\text { mass }(\mathrm{kg})\end{array}$ & $\begin{array}{c}\text { Head impact mass } \\
(\text { ratio })\end{array}$ \\
\hline WAD & $120.2 \pm 12.1$ & $12.9 \pm 3.8$ & $62.2 \pm 10.2$ & $3.6 \pm 0.6$ & $0.95 \pm 0.18$ \\
$\begin{array}{c}\text { 1750-1900 } \\
\text { WAD }\end{array}$ & $129.5 \pm 10.7$ & $15.2 \pm 2.7$ & $55.0 \pm 8.7$ & $4.0 \pm 0.4$ & $0.92 \pm 0.06$ \\
$\begin{array}{c}\text { 1900-2050 } \\
\text { WAD }\end{array}$ & $138.8 \pm 10.5$ & $14.9 \pm 2.6$ & $52.3 \pm 5.5$ & $4.6 \pm 0.5$ & $0.93 \pm 0.07$ \\
2050-2200 & & &
\end{tabular}

\subsubsection{Comparison between Impactor Response in Sub-system Test and Head Response in Full- scale Impact}

Impactor simulations predicted much lower HIC values $(1257 \pm 647)$ than full-scale pedestrian impact simulations (2016 \pm 1087), and the $\mathrm{p}<0.001$ in paired-T tests indicated significant differences. The difference between the impact types was more significant in the nonlateral impact scenarios (HIC of impactor tests: $1348 \pm 675$; full-scale:2539 \pm 993) (k=0.53; $\mathrm{p}<0.001)$ than the lateral impacts (impactor: $1135 \pm 610$; full-scale:1318 \pm 786) $(\mathrm{k}=0.79 ; \mathrm{p}=$ $0.22)$. 

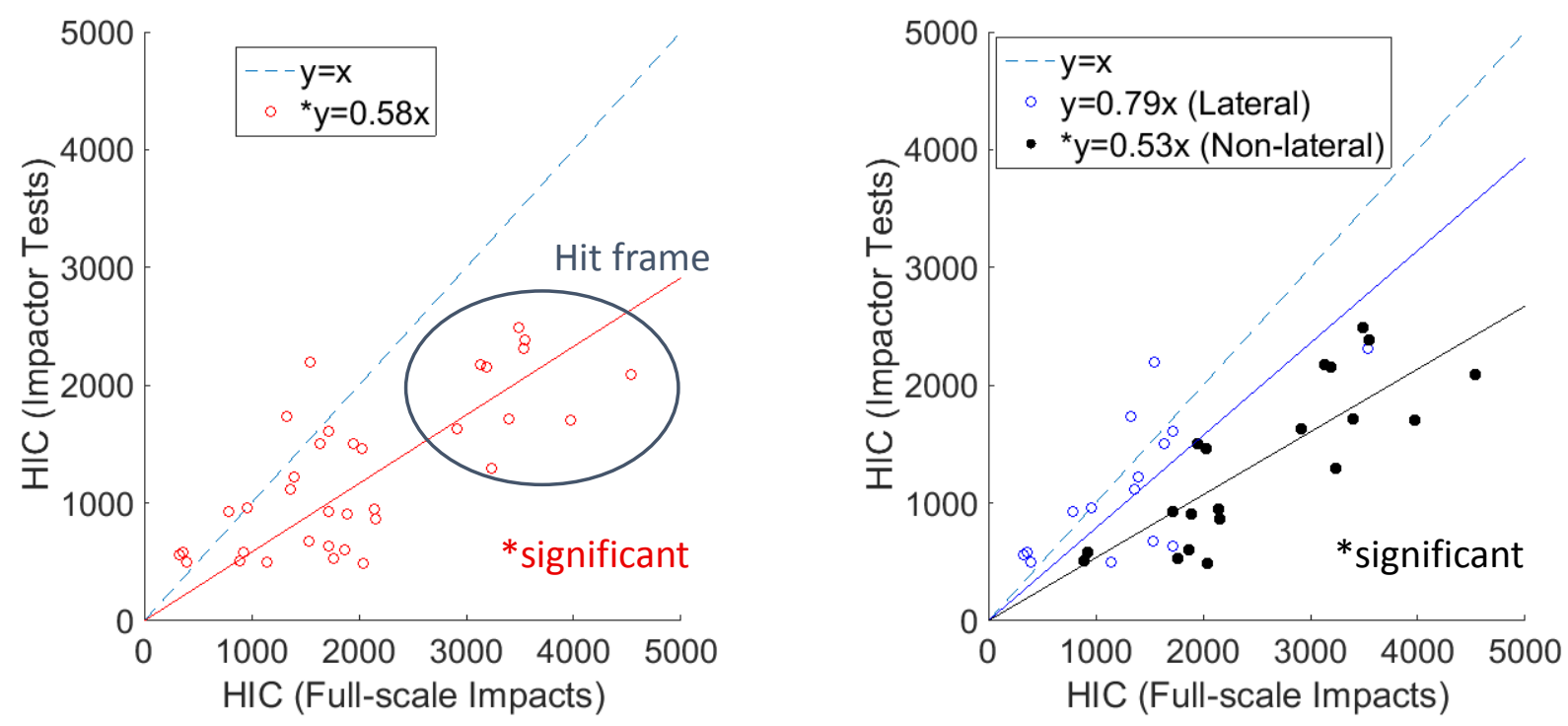

Figure 4.8 Comparison on HIC between impactor tests and full-scale impacts (Left - All impact scenarios; Right - Lateral and non-lateral scenarios)

\subsubsection{Hypothesis Testing - Effect of Vehicle Design}

Small differences were observed on BrIC in both configurations. In impact configuration 1 , the BrICs in all 12 baseline simulations are $1.386 \pm 0.201$, while in the simulations using softer windshield material the BrICs are $1.384 \pm 0.196$. In impact configuration 2, the BrIC obtained in all 11 baseline simulations are $1.601 \pm 0.183$, while in the simulations using varied vehicle stiffness obtained $1.605 \pm 0.209$.

In 5 of the 6 vehicle stiffness variations, the paired-T tests obtained p values higher than 0.05 , which also suggested the BrIC is generally insensitive to these vehicle stiffness variations. 


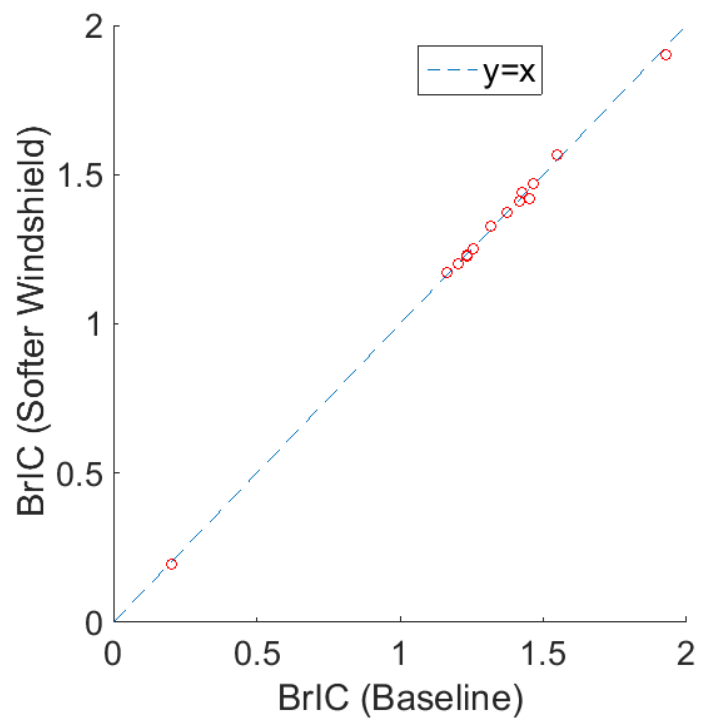

Softer windshield material $(\mathrm{p}=0.675)$

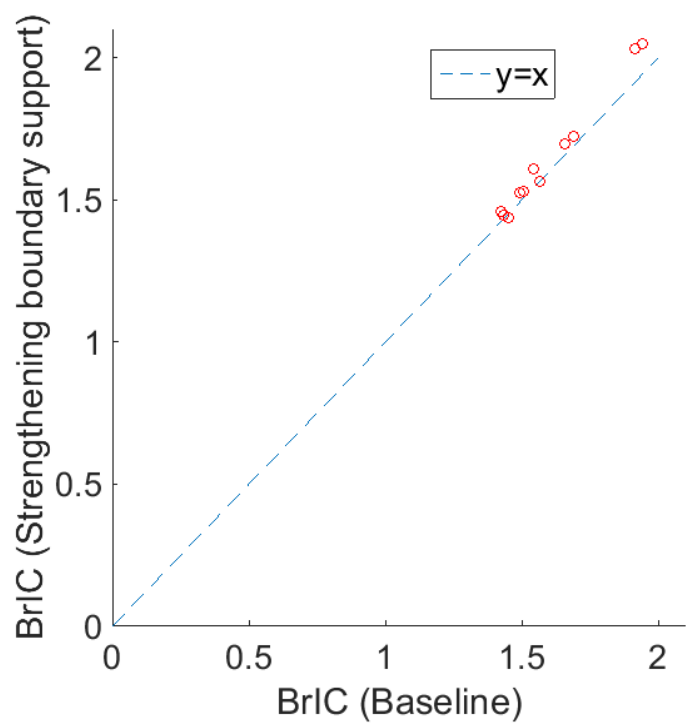

Strengthening hinge support $(\mathrm{p}=0.007)$

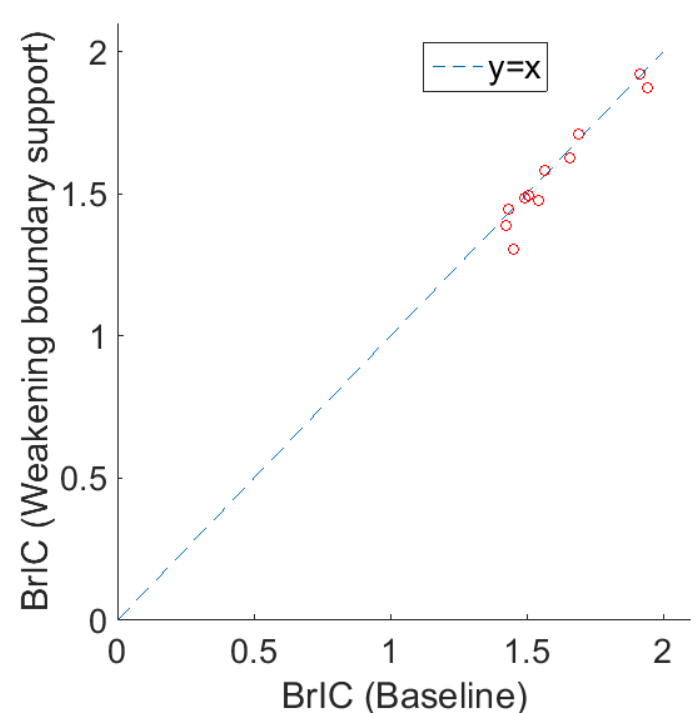

Weakening hinge support $(p=0.101)$

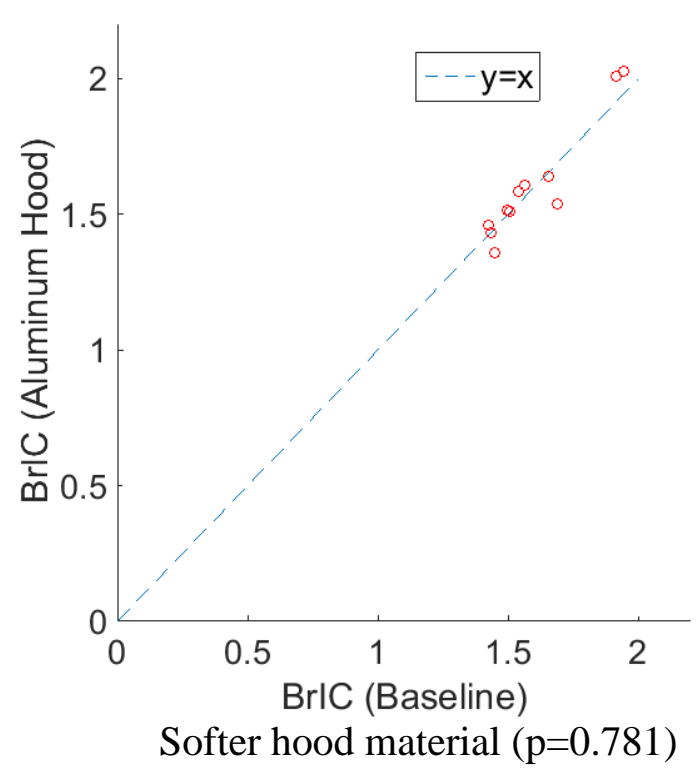




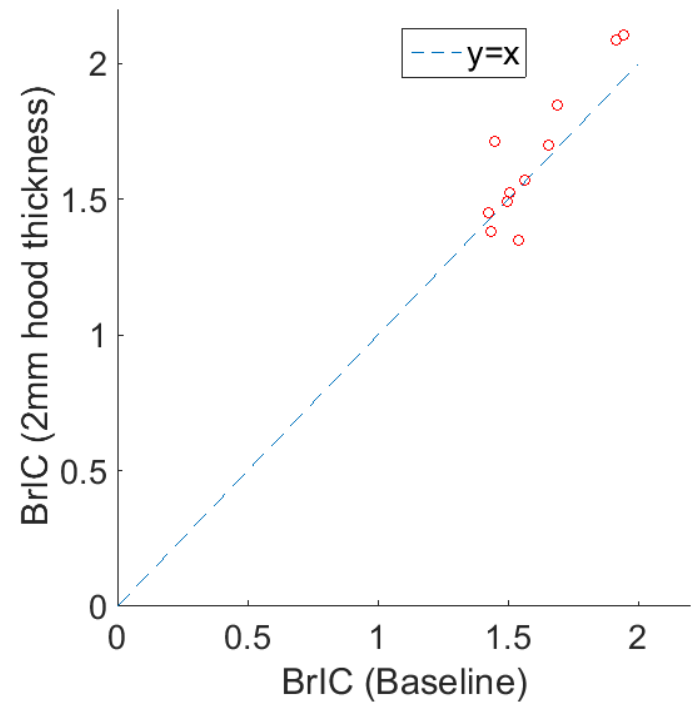

Increasing hood thickness $(\mathrm{p}=0.173)$

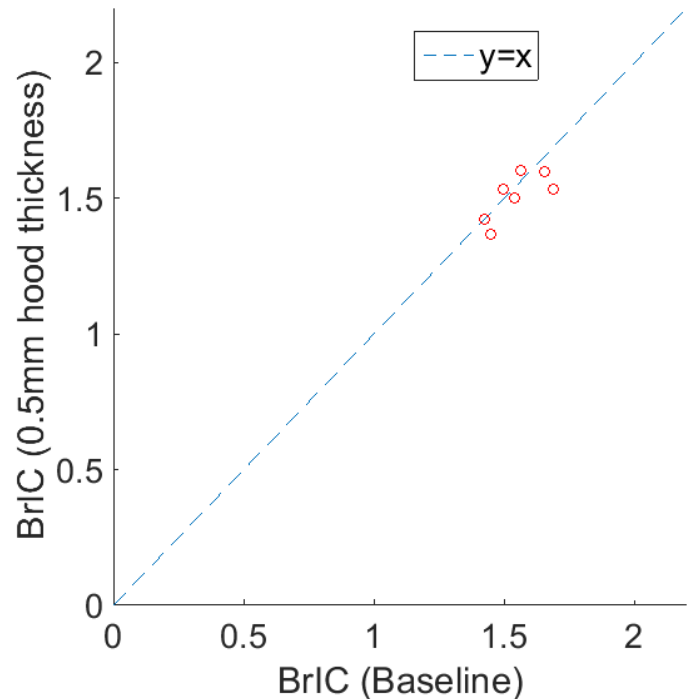

Decreasing hood thickness $(\mathrm{p}=0.216)$

Figure 4.9 Effect of varied vehicle stiffness design on BrIC

\section{Varying Windshield Material}

Softer windshield obtains lower HIC in both full-scale impacts and impactor tests, and in both full-scale impacts and the impactor tests the $\mathrm{p}<0.001$, suggesting softer windshield caused significantly different HIC on impactors and heads.

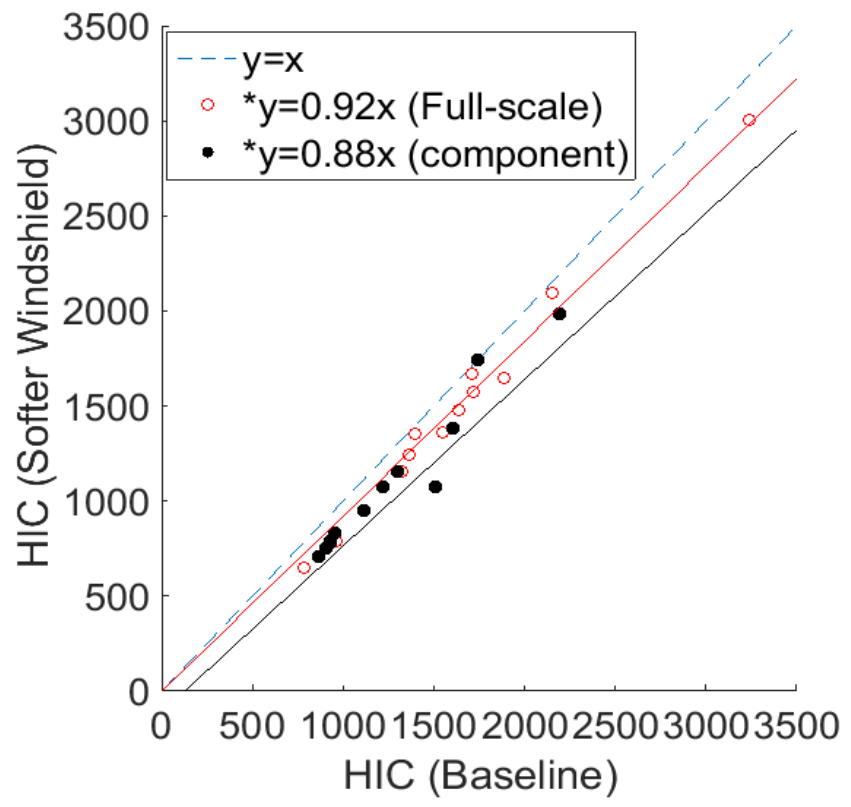


Figure 4.10 Effect of windshield material on HIC
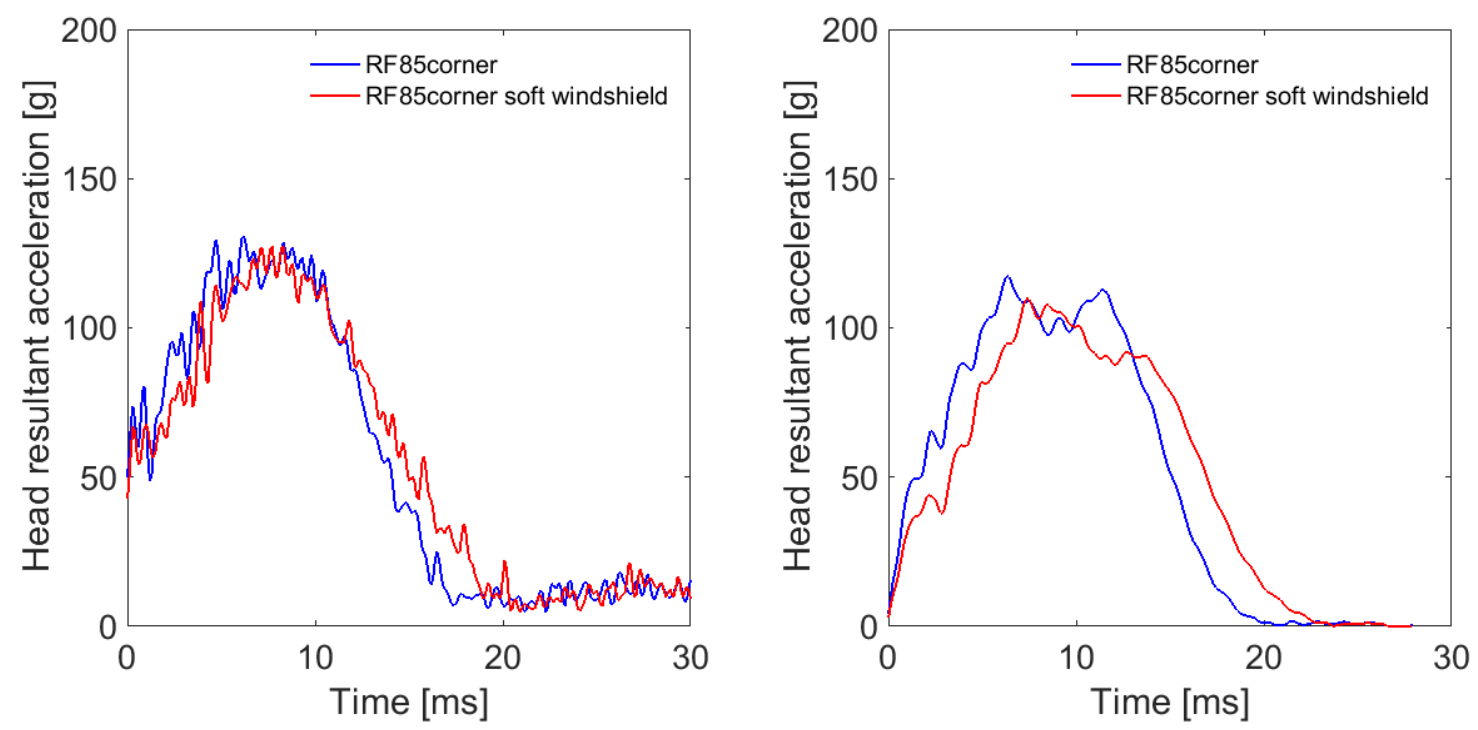

Figure 4.11 Time history of resultant head acceleration of RF85corner (Left - full-scale impact; Right impactor test)

\section{Varying Hood Thickness}

When increasing the hood thickness, the HIC increased in both impactor tests and fullscale impacts. In both full-scale impacts and the impactor tests the $\mathrm{p}<0.001$, suggesting increasing hood thickness caused significantly different HIC on impactors and heads. 


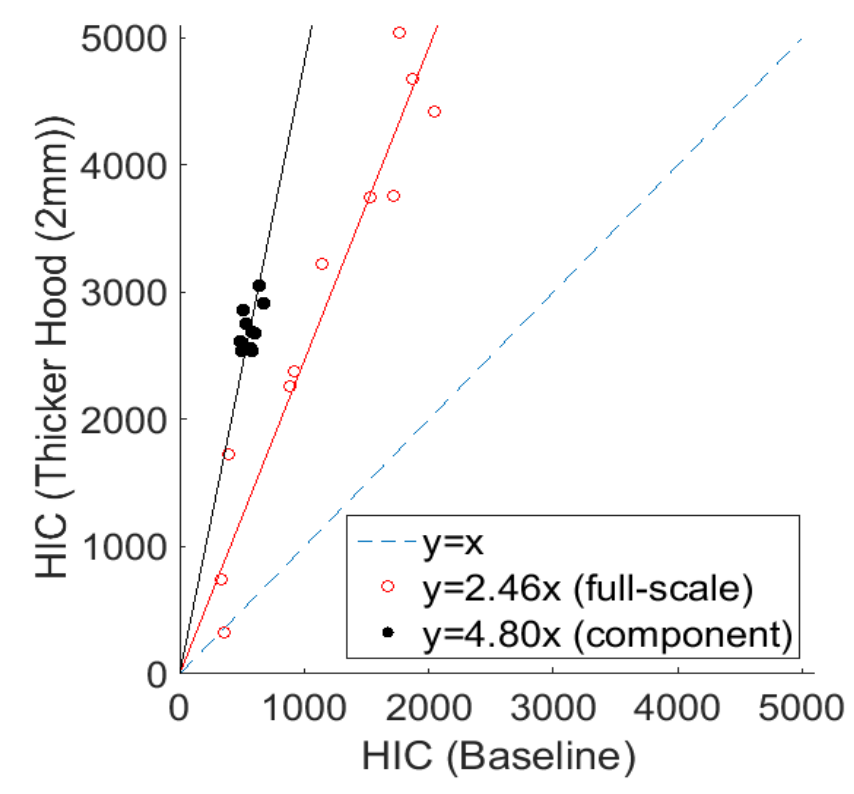

Figure 4.12 Effect of increasing hood thickness on HIC
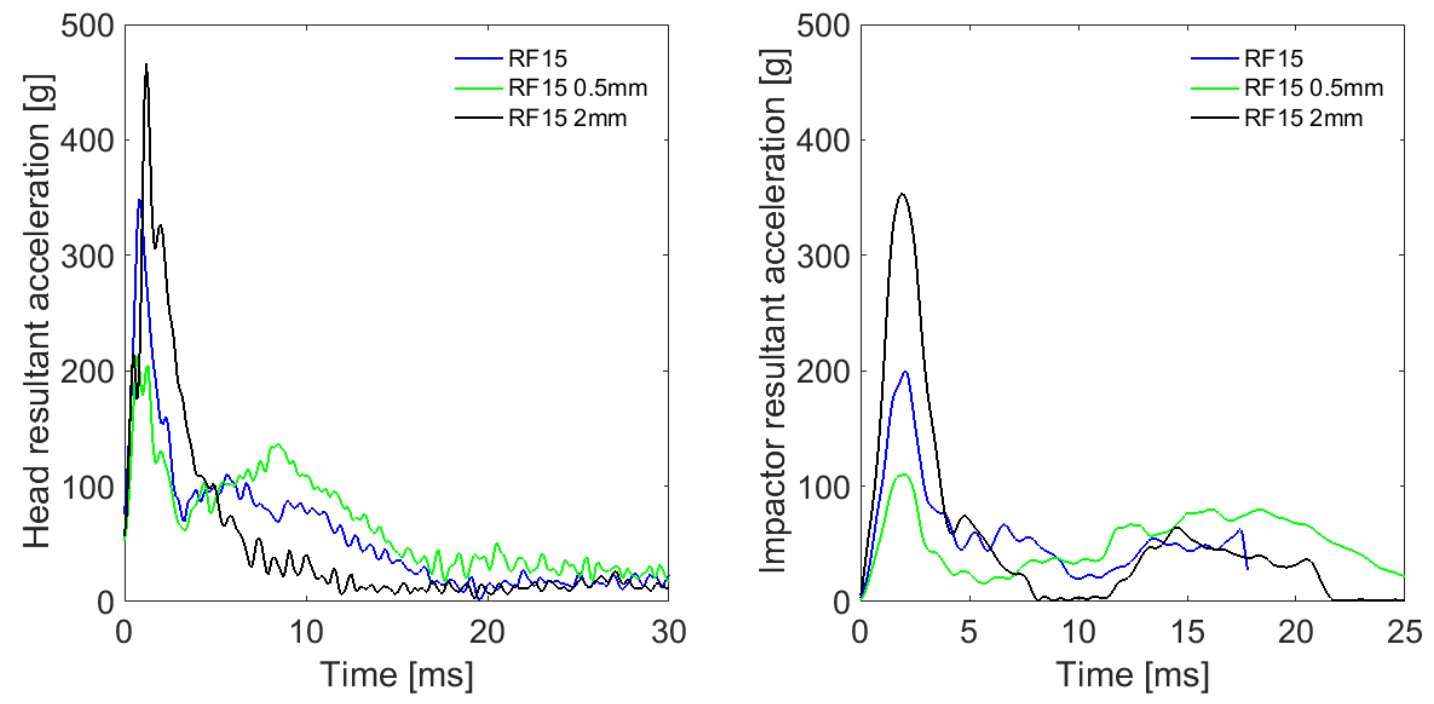

Figure 4.13 Time history of head acceleration when varying hood thickness of RF15 (Left - full-scale impact; Right - impactor test)

When decreasing the hood thickness, the $\mathrm{p}$ values are higher than 0.05 in full-scale impacts (all impact scenarios: $\mathrm{p}=0.128$; lateral impacts: $\mathrm{p}=0.299$ ), while lower than 0.05 in impactor tests (all impact scenarios: $\mathrm{p}<0.001$; lateral impacts: $\mathrm{p}=0.001$ ). The HIC decreased 
more in impactor tests (slope $k=0.63)$ than in full-scale impacts $(k=0.78)$, especially in lateral impact scenarios (impactors tests: $\mathrm{k}=0.57$; full-scale impacts: $\mathrm{k}=0.96$ ).
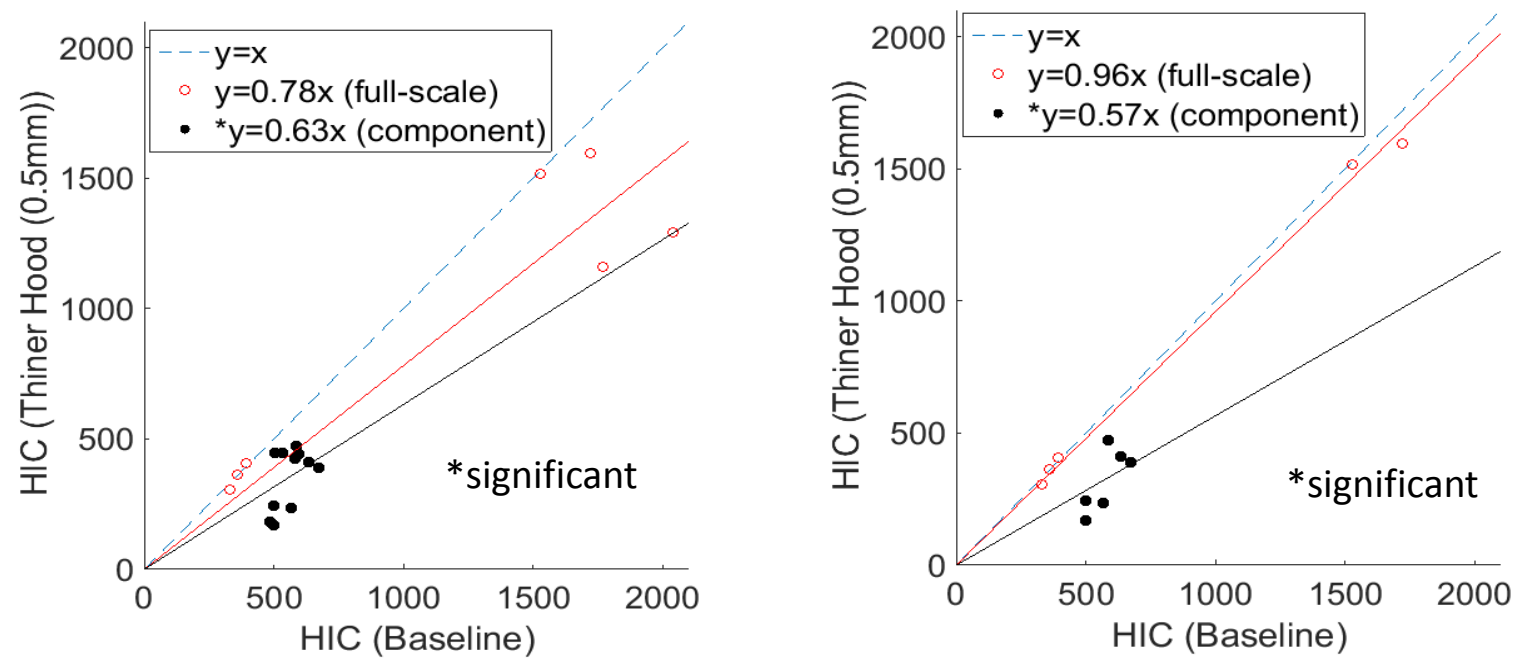

Figure 4.14 Effect of decreasing hood thickness on HIC (Left - All impact scenarios; Right - Lateral impact scenarios)

\section{Varying Hood Material}

When decreasing the material stiffness, the $\mathrm{p}$ values are higher than 0.05 in full-scale impacts (all impact scenarios: $\mathrm{p}=0.226$; lateral impacts: $\mathrm{p}=0.119$ ), while lower than 0.05 in impactor tests (all impact scenarios: $\mathrm{p}=0.003$; lateral impacts: $\mathrm{p}=0.01$ ). The HIC significantly decreased in impactor tests (slope $k=0.79)$ but did not decrease in full-scale impacts $(k=1.02)$, especially in lateral impact scenarios (impactors tests: $k=0.71$; full-scale impacts: $k=1.07$ ). 

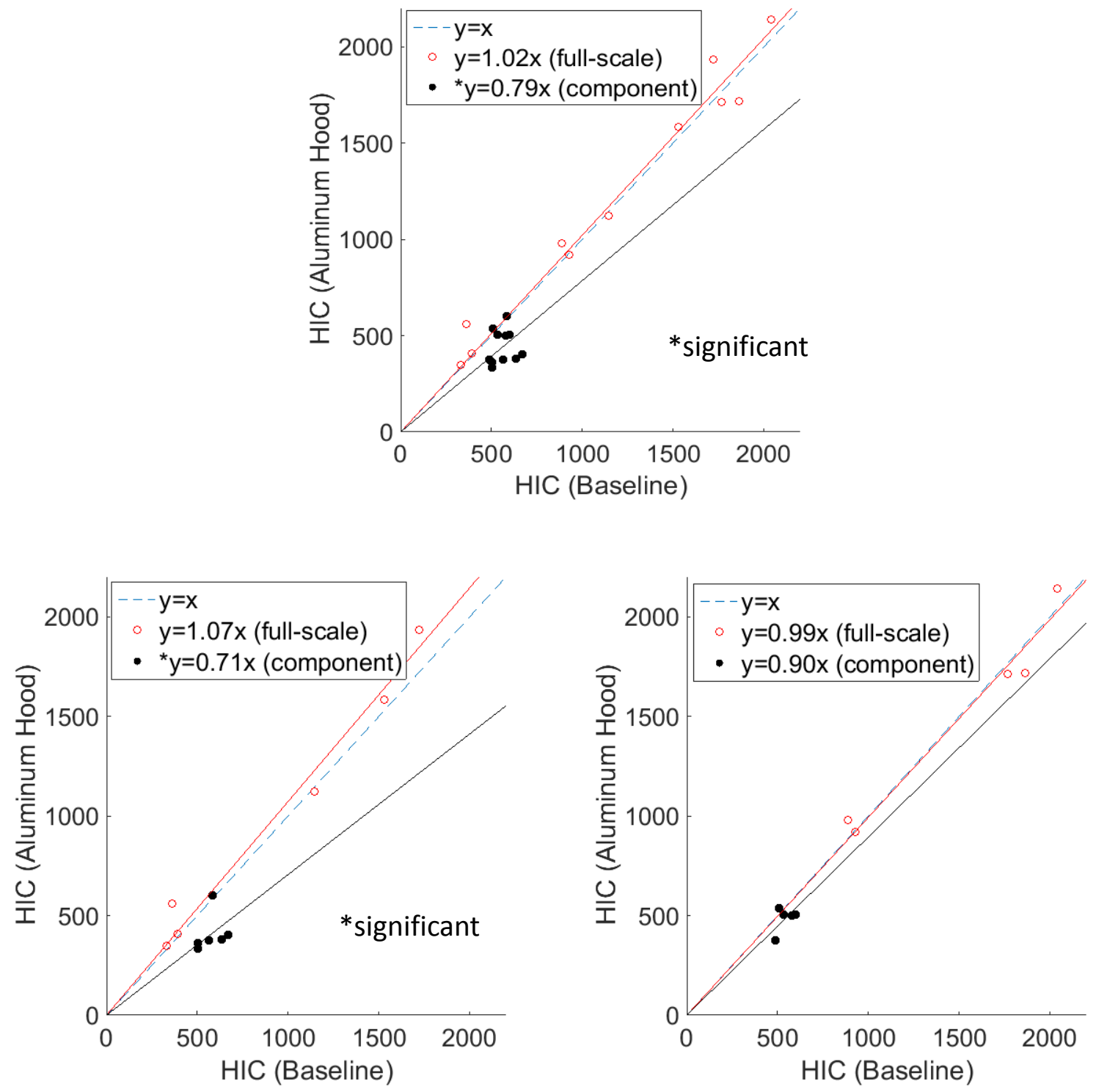

Figure 4.15 Effect of hood material on HIC (Upper - All impact scenarios; Lower Left - lateral impact scenarios; Lower Right - non-lateral impact scenarios) 

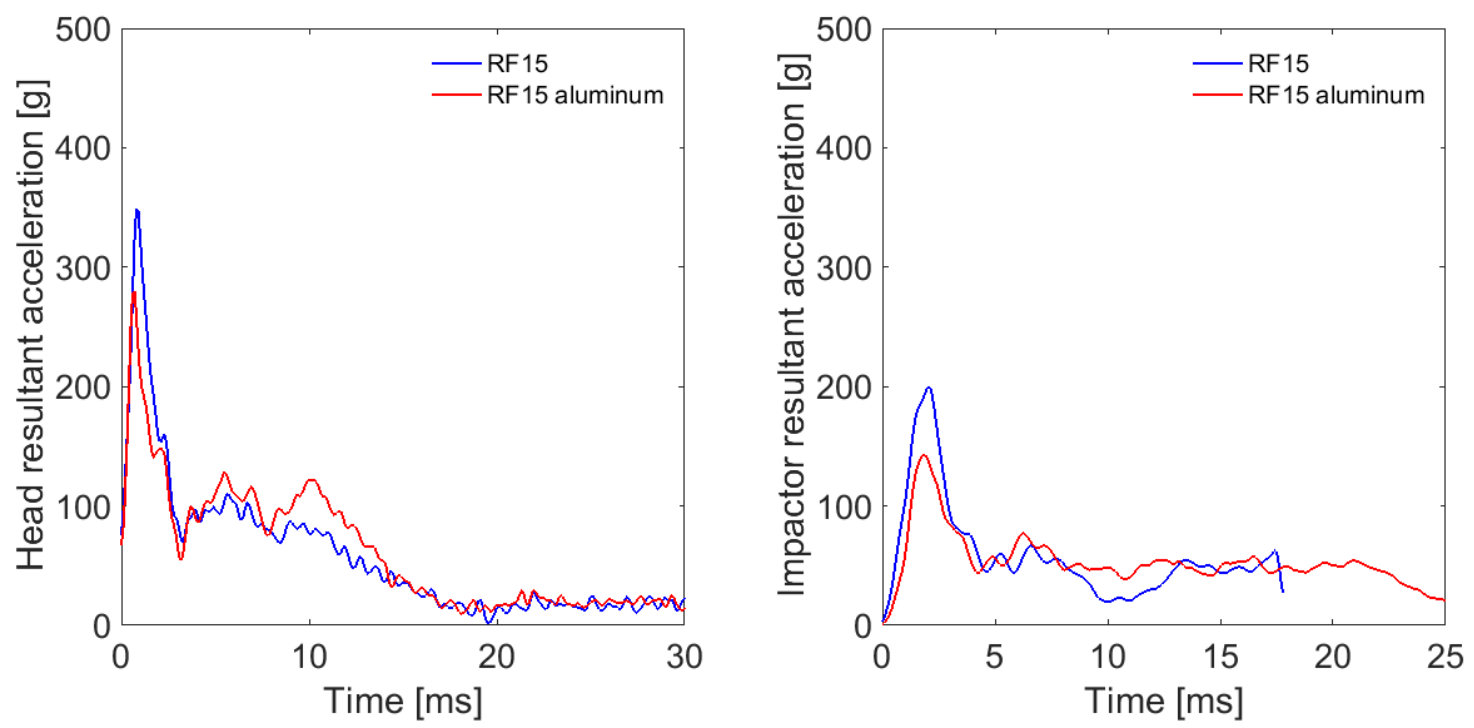

Figure 4.16 Time history of head acceleration when varying material stiffness of RF15 (Left - full-scale impact; Right - impactor test)

\section{Varying Hood Support}

When weakening the hood hinge supports to the extent of this study's, the mean difference is not significant in full-scale impacts $(p=0.17)$ but is significant in impactor tests ( $p=0.047$ ); while when strengthening the hood hinge supports, the mean difference is significant in full-scale impacts $(p=0.001)$, but not significant in impactor tests $(p=0.765)$.

Either weakening or strengthening the hinge supports, the HIC changed less in impactor tests (weakening: $\mathrm{k}=1.01$; strengthening: $\mathrm{k}=1.0$ ) than in full-scale impacts (weakening: $\mathrm{k}=0.95$; strengthening: $\mathrm{k}=1.2$ ). 

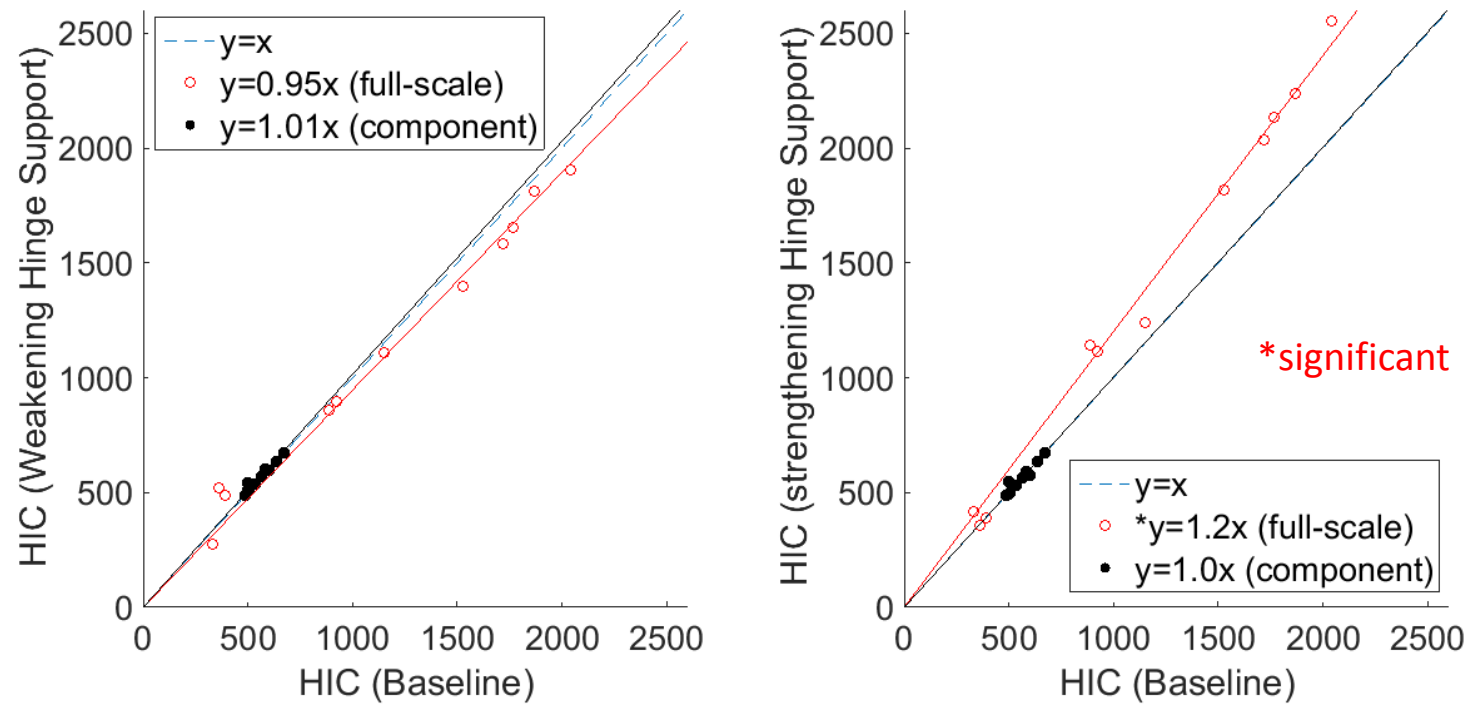

Figure 4.17 Effect of hinge support on HIC (Left - weakening support; Right - strengthening support)
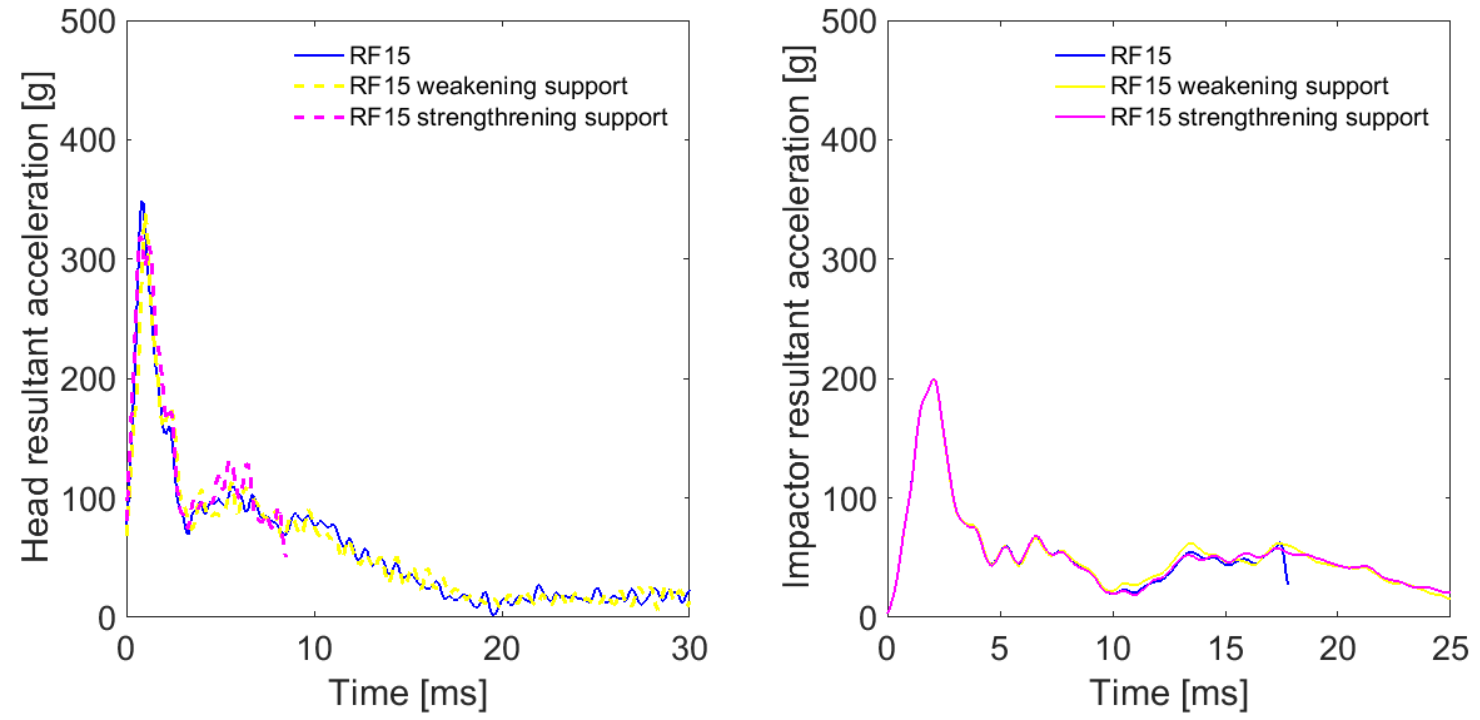

Figure 4.18 Time history of head acceleration when varying hood hinge support of RF15 (Left - full-scale impact; Right - impactor test) 


\subsection{Discussion}

\subsubsection{Pedestrian Head Impact Conditions in Full-scale Impact}

\section{Initial Direction dependence}

The head impact speeds observed in lateral impacts were comparable with previous studies (Mizuno 2005; Chen et al. 2009) and the current Euro-NCAP test procedure which is using $11.1 \mathrm{~m} / \mathrm{s}$ as input conditions. However, the non-lateral impacts caused 54.2\% higher head impact speeds, which have been ignored in the previous studies, account for $28 \%$ impact scenarios in the real-world (Chidester et al. 2001; Neal-Sturgess et al. 2007). The main reason for lower head impact speeds in lateral impacts could be more shoulder impact and elbow impacts in lateral impact which delays the head impact timing, and caused the neck in tension and thus reduces the head impact force and head impact speed.

In lateral impacts, the head effective mass is around 0.8 times of head original mass, while in non-lateral impact it is around 1.0 times. This suggests less role of the neck in the nonlateral impact. In the lateral impacts, generally the shoulder impacts the vehicle first, which then results in the neck being in tension when the head impacts the vehicle, and this reduces the head impact force with the vehicle compared to the situation without the neck interaction, and thus reduces the head effective mass. While in the non-lateral impacts, there is less or no shoulder impacts, and there are less time difference between upper body impacting the vehicle and head impacting the vehicle, so the head-neck interaction is less significant and the head effective mass was found to be almost identical to head original mass. 


\section{$\underline{\text { Location dependence }}$}

Higher head impact speeds were observed in higher WAD areas, but this saturated in the windshield area. A possible reason for this saturation could be the steep angle of the windshield which could earlier the head impact timing and thus reduce the head impact speed.

The increase of head effective mass was observed with the higher WAD, but this was mainly caused by the anthropometry difference. The taller the pedestrian, the higher the WAD that will be obtained, however in this study a taller pedestrian was given higher body mass and head mass to represent the general population.

\subsubsection{Comparison between Impactor Response and Head Response}

The impactor tests predicted lower HIC values than the full-scale impact, and the difference was caused mainly from the non-lateral impact scenarios which were found to have higher head impact speed. However, this does not necessarily mean the kinematical difference was the only reason for the impactor tests not capturing the head response in full-scale impact. Other factors such as the head-neck interaction, vehicle deformation, head rotational kinematics, model difference, difference between head mass and impactor mass, and head contact geometry could all play roles leading to different responses on the impactor and head, and the effect of these factors needs to be further investigated.

\subsubsection{Hypothesis Testing - Effect of Vehicle Stiffness Design Rotational Injury Measurement}

In this study, in full-scale impacts, in both impact configuration 1 without vehicle predeformation caused by torso before head impact and impact configuration 2 with vehicle predeformation, BrIC was not sensitive to these vehicle stiffness variations. BrIC is a kinematical 
injury criteria based on the maximum head angular velocity in three orthogonal components. In this study, this metric reached the peak values before the head impacted the vehicle. Previous studies showed vehicle front geometry affects head kinematics (Liu et al. 2002; Chen et al. 2009; Han et al. 2012; Watanabe et al. 2012). In this study only vehicle stiffness in head impact areas were varied while the front geometry was unchanged. This resulted in the insensitivity of head kinematics before the head impact, and thus led to the insensitivity of BrIC.
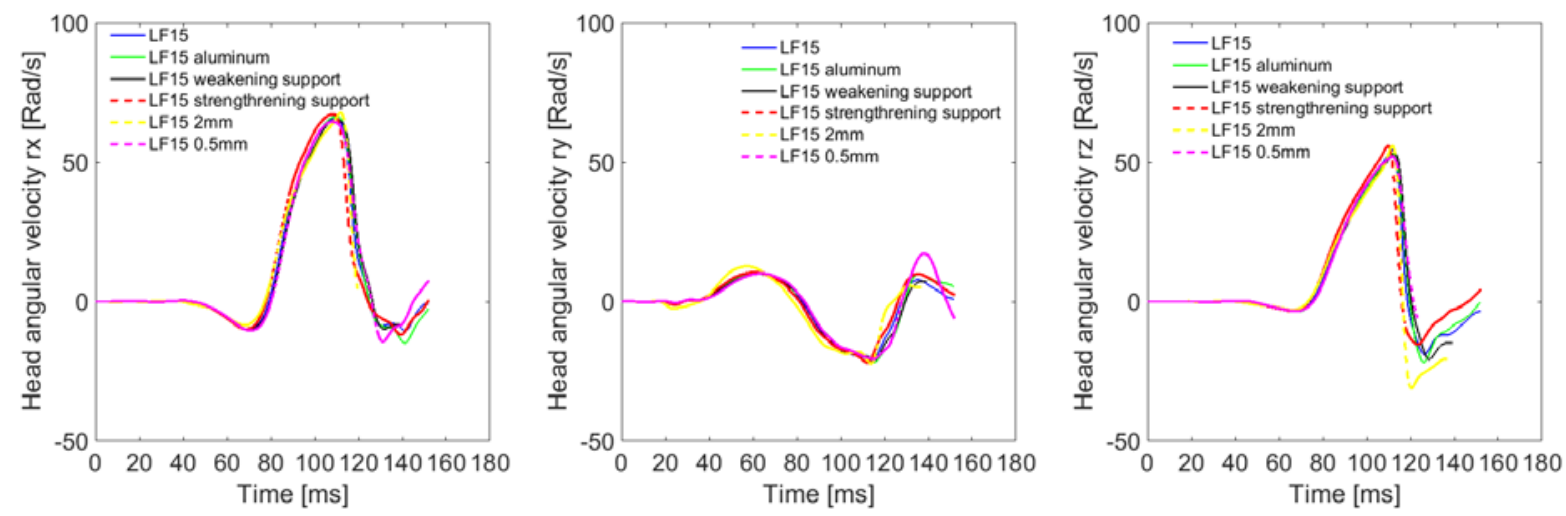

Figure 4.19 Time history of angular velocity of LF15 (Left - rx; Middle - ry; Right - rz)

\section{$\underline{\text { Translational Injury Measurement }}$}

The sensitivity of head or impactor responses depend on the extent of change of stiffness, but for same variation of vehicle stiffness, different $\mathrm{p}$ values and $\mathrm{k}$ values indicated different sensitivity levels. In impact configuration 1, when varying windshield material stiffness, no major difference was observed on the $\mathrm{p}$ values and $\mathrm{k}$ values.

However, in impact configuration 2, when decreasing the hood thickness or hood material stiffness, lower $\mathrm{p}$ values and $\mathrm{k}$ values suggested these vehicle design changes had greater effect on the impactor response than the head response. Furthermore, when strengthening the hood hinge supports, lower $\mathrm{p}$ values and higher $\mathrm{k}$ values suggested these vehicle design changes had greater effect on the head response than the impactor response. 
Softer windshield obtains lower HIC in both full-scale impact and impactor tests. This suggests that when there is no vehicle pre-deformation caused by torso on head impact area, the vehicle stiffness design variation had a similar influence on impactor response and head response in full-scale impact.

However, when there is vehicle pre-deformation, in impact configuration 2, the decreases on hood thickness or material stiffness resulted in lower HIC on impactors, but not necessarily resulted in lower HIC on full-scale head impacts. A notable reason was found in the time history curves. Specifically, there is always a second peak on acceleration (Figure 4.35 - Figure 4.33) when more vehicle deformation was caused by the softer or thinner hood, and this second peak resulted in higher HIC values though the peak acceleration was lower.

The head response was more sensitive than the impactor response on the hood hinge mounting support, and the reason could be less involvement of the hinge in impactor tests than the full-scale impact. The effective mass of the whole upper body in the full-scale impact could be higher than the $4.5 \mathrm{~kg}$ mass of head impactor, which produced higher impact energy and more vehicle deformation, and thus caused more involvement of hinge support in the impact course. This issue needs to be further investigated.

The capability of HIC to predict head injury risk has long been debated. The HIC was developed from the Wayne State Concussive Tolerance Curve (Gadd 1966.), which showed that the linear acceleration required for skull fracture is inversely related to impact duration. Since skull fracture was correlated with moderate concussion, the WSTC was proposed as a predictor for head injury. A power of 2.5, which was based on the slope of the approximated Gadd's Line on the WSTC, was given to the average acceleration, and then multiplied by the time duration, 
and was maximized in $15 \mathrm{~ms}$ or $36 \mathrm{~ms}$ to calculate the HIC. In the current pedestrian regulation and Euro-NCAP test, the 15 ms time window is used.

$$
H I C=\left\{\left(t_{2}-t_{1}\right)\left[\frac{1}{t_{2}-t_{1}} \int_{t_{1}}^{t_{2}} a(t) d t\right]^{2.5}\right\}_{\max }
$$

Force was not a good indicator to predict injury risk, because it also depends on the contact area. Compared to the peak force, the HIC includes the effect of time duration, and removes the effect of head mass. The softer or thinner hood design increases the hood deformation and was found to cause a second peak on the acceleration curve probably due to more involvement of the hood inner, and this could potentially increase the HIC values. Also, the deformation itself could stiffer the hood and thus increase the peak acceleration and the HIC values, but this needs to be studied and verified in the next steps.

\section{Next Step}

The next step of this study is to find the reasons for the observations in this chapter regarding why the impactor tests cannot accurately capture the head response in full-scale impacts, and why vehicle stiffness design variation does not have the same effect on the impactor response and head response. As discussed above, these potential reasons for impactor tests not capturing the full-scale head response include differences on kinematics, differences on methodology such as missing head-neck interaction, vehicle deformation, and head rotational kinematics, or differences in the model itself such as mass and contact geometry.

\subsection{Conclusion}

In this chapter, the impactor response and pedestrian head response in the full-scale vehicle-pedestrian impacts were compared in diverse impact scenarios, and then the hypothesis 
that vehicle stiffness design variations have different influences on the impactor response and head response was tested. The conclusions are summarized here.

Non-lateral impact caused higher head impact speeds than lateral impact. Pedestrian head impact conditions were verified to be location-dependent. Impactor tests underestimated the HIC compared to full-scale impacts, and this observation is more from non-lateral impact scenarios.

Full-scale impact was more complicated than component tests. The head rotational-based injury risk based on BrIC was found to be insensitive to vehicle stiffness variation in this study.

The current findings suggest that the difference between the impactor tests and full-scale impact may depend on the impact configurations. When there was no vehicle pre-deformation on the head impact area, the vehicle stiffness design variation does have similar influence on the impactor response and head response. However, when there is vehicle pre-deformation, the vehicle stiffness design variation could have different influence on the impactor response and head response, especially in lateral impact scenarios. 


\subsection{Appendix for Chapter 4}

\subsubsection{Overall Output of All Simulations}

Table 4.4 Head response in full-scale impact and impactor response in sub-system tests

\begin{tabular}{|c|c|c|c|c|c|c|c|c|c|c|c|c|c|c|c|c|}
\hline \multirow[b]{2}{*}{ No. } & \multicolumn{14}{|c|}{ Full-scale Impact } & \multicolumn{2}{|c|}{$\begin{array}{c}\text { Sub-system } \\
\text { test }\end{array}$} \\
\hline & $\begin{array}{l}\text { Vehicle } \\
\text { design }\end{array}$ & $\begin{array}{c}\text { Impact } \\
\text { configuration }\end{array}$ & Posture & Anthropometry & Direction & Location & $\begin{array}{l}\text { Head } \\
\text { impact } \\
\text { timing }\end{array}$ & $\begin{array}{c}\text { Head } \\
\text { impact } \\
\text { speed }\end{array}$ & $\begin{array}{c}\text { Head } \\
\text { impact } \\
\text { angle }\end{array}$ & $\begin{array}{c}\text { Wrap around } \\
\text { distance }\end{array}$ & $\begin{array}{l}\text { Head } \\
\text { effective } \\
\text { mass }\end{array}$ & BrIC & HIC & $\begin{array}{l}\text { Peak } \\
\text { force }\end{array}$ & HIC & $\begin{array}{l}\text { Peak } \\
\text { force }\end{array}$ \\
\hline 1 & 0 & 2 & LF & 15 & 0 & 1 & 114.5 & 12.52 & 65.01 & 1776.3 & 3.075 & 1.656 & 1529 & 8.8 & 672 & 8.1 \\
\hline 2 & 2 & 2 & LF & 15 & 0 & 1 & 114.3 & 12.98 & 64.22 & - & 2.995 & 1.64 & 1585 & 4.8 & 402 & 4.5 \\
\hline 3 & 3 & 2 & LF & 15 & 0 & 1 & 114.5 & 12.88 & 64.77 & - & 3.043 & 1.665 & 1644 & 6.4 & 476 & 5.7 \\
\hline 4 & 4 & 2 & LF & 15 & 0 & 1 & 114.3 & 12.66 & 64.41 & - & 3.007 & 1.646 & 1470 & 7 & 512 & 7.4 \\
\hline 5 & 5 & 2 & LF & 15 & 0 & 1 & 115.6 & 12.355 & 64.18 & - & 3.045 & 1.627 & 1397 & 7.9 & 671 & 8.1 \\
\hline 6 & 6 & 2 & LF & 15 & 0 & 1 & 112.4 & 12.75 & 65.93 & - & 3.092 & 1.695 & 1820 & 9.7 & 674 & 8.1 \\
\hline 7 & 7 & 2 & LF & 15 & 0 & 1 & 114.6 & 13.158 & 65.26 & - & 3.086 & 1.599 & 1518 & 4.6 & 391 & 4.5 \\
\hline 8 & 8 & 2 & LF & 15 & 0 & 1 & 114.2 & 11.62 & 63.72 & - & 3.405 & 1.701 & 3748 & 12.9 & 2915 & 14.6 \\
\hline 9 & 9 & 2 & LF & 15 & 0 & 1 & 114.7 & 11.81 & 64.42 & - & 3.202 & 1.735 & 2964 & 12.3 & 1808 & 12.1 \\
\hline 10 & 10 & 2 & LF & 15 & 0 & 1 & 114.2 & 12.0 & 63.66 & - & 3.347 & 1.701 & 2876 & 11.2 & 1462 & 10.5 \\
\hline 11 & 0 & 2 & LF & 15 & 90 & 1 & 118.9 & 16.23 & 57.24 & 1834.1 & 3.987 & 1.508 & 1865 & 7.6 & 598 & 6.7 \\
\hline 12 & 2 & 2 & LF & 15 & 90 & 1 & 119.3 & 16.49 & 57.62 & - & 3.878 & 1.511 & 1719 & 4.8 & 506 & 3.5 \\
\hline 13 & 5 & 2 & LF & 15 & 90 & 1 & 119.6 & 16.29 & 57.24 & - & 3.997 & 1.495 & 1810 & 8.1 & 596 & 6.7 \\
\hline 14 & 6 & 2 & LF & 15 & 90 & 1 & 117.9 & 16.17 & 58.0 & - & 3.951 & 1.529 & 2238 & 7.8 & 575 & 6.7 \\
\hline 15 & 7 & 2 & LF & 15 & 90 & 1 & - & - & - & - & - & - & - & - & 444 & 3.7 \\
\hline 16 & 8 & 2 & LF & 15 & 90 & 1 & 119.8 & 15.69 & 58.28 & - & 4.021 & 1.526 & 4675 & 14.9 & 2674 & 14.4 \\
\hline 17 & 0 & 2 & LF & 15 & n90 & 1 & 117.5 & 15.52 & 51.56 & 1833.9 & 4.224 & 1.915 & 926 & 4.9 & 581 & 6.8 \\
\hline 18 & 2 & 2 & LF & 15 & n90 & 1 & 117.4 & 15.47 & 51.42 & - & 4.178 & 2.009 & 918 & 3.9 & 500 & 3.4 \\
\hline 19 & 5 & 2 & LF & 15 & n90 & 1 & 118.1 & 15.55 & 51.52 & - & 4.228 & 1.927 & 899 & 4.6 & 602 & 6.8 \\
\hline 20 & 6 & 2 & LF & 15 & n90 & 1 & 116.6 & 15.48 & 52.25 & - & 4.261 & 2.032 & 1114 & 5.2 & 590 & 6.8 \\
\hline 21 & 7 & 2 & LF & 15 & n90 & 1 & 117.7 & 15.68 & 51.70 & - & 4.002 & - & - & - & 423 & 3.8 \\
\hline 22 & 8 & 2 & LF & 15 & $\mathrm{n} 90$ & 1 & 117.4 & 15.76 & 54.29 & - & 4.462 & 2.088 & 2384 & 9.4 & 2686 & 14.5 \\
\hline 23 & 0 & 2 & RF & 15 & 0 & 1 & 110.9 & 13.55 & 64.46 & 1783 & 3.033 & 1.688 & 1720 & 10.1 & 635 & 8.1 \\
\hline 24 & 2 & 2 & RF & 15 & 0 & 1 & 111.2 & 14.43 & 62.34 & - & 3.183 & 1.537 & 1936 & 5.7 & 382 & 4.4 \\
\hline 25 & 3 & 2 & RF & 15 & 0 & 1 & 111.1 & 14.01 & 63.33 & - & 3.052 & 1.603 & 1750 & 7.6 & 447 & 5.8 \\
\hline 26 & 4 & 2 & RF & 15 & 0 & 1 & 111.3 & 13.78 & 63.54 & - & 3.103 & 1.61 & 1598 & 8.5 & 457 & 7.1 \\
\hline 27 & 5 & 2 & RF & 15 & 0 & 1 & 112.0 & 13.44 & 64 & - & 3.047 & 1.709 & 1586 & 9.7 & 634 & 8.1 \\
\hline 28 & 6 & 2 & RF & 15 & 0 & 1 & 109.0 & 13.95 & 65.64 & - & - & 1.723 & 2037 & 10.7 & 637 & 8.1 \\
\hline 29 & 7 & 2 & RF & 15 & 0 & 1 & 110.5 & 14.82 & 61.47 & - & 3.534 & 1.532 & 1596 & 6.3 & 412 & 4.7 \\
\hline 30 & 8 & 2 & $\mathrm{RF}$ & 15 & 0 & 1 & 112.6 & 11.93 & 59.79 & - & 3.091 & 1.848 & 3758 & 14 & 3057 & 15.2 \\
\hline 31 & 9 & 2 & $\mathrm{RF}$ & 15 & 0 & 1 & 112.1 & 12.68 & 64.27 & - & 3.17 & 1.788 & 3217 & 13.7 & 1773 & 12.2 \\
\hline 32 & 10 & 2 & RF & 15 & 0 & 1 & 112.6 & 12.41 & 63.29 & - & 3.225 & 1.732 & 2333 & 10.9 & 1451 & 10.9 \\
\hline 33 & 0 & 2 & RF & 15 & 90 & 1 & 119.8 & 16.23 & 56.97 & 1823.7 & 4.028 & 1.494 & 1767 & 7.6 & 533 & 7 \\
\hline 34 & 2 & 2 & RF & 15 & 90 & 1 & 120.0 & 16.44 & 56.48 & - & 3.874 & 1.517 & 1712 & 4.3 & 504 & 3.7 \\
\hline
\end{tabular}




\begin{tabular}{|c|c|c|c|c|c|c|c|c|c|c|c|c|c|c|c|c|}
\hline 35 & 5 & 2 & $\mathrm{RF}$ & 15 & 90 & 1 & 120.4 & 16.26 & 57.04 & - & 4.026 & 1.484 & 1656 & 7.7 & 537 & 7 \\
\hline 36 & 6 & 2 & $\mathrm{RF}$ & 15 & 90 & 1 & 118.9 & 16.10 & 57.71 & - & 3.987 & 1.526 & 2132 & 7.9 & 529 & 7 \\
\hline 37 & 7 & 2 & $\mathrm{RF}$ & 15 & 90 & 1 & 119.8 & 16.43 & 56.67 & - & 3.887 & 1.534 & 1161 & 4.2 & 447 & 4 \\
\hline 38 & 8 & 2 & $\mathrm{RF}$ & 15 & 90 & 1 & 120.6 & 15.67 & 57.74 & - & 4.048 & 1.493 & 5037 & 15.1 & 2753 & 14.7 \\
\hline 39 & 0 & 2 & $\mathrm{RF}$ & 15 & n90 & 1 & 117.4 & 15.40 & 51.04 & 1834.1 & 4.322 & 1.944 & 888 & 4.4 & 506 & 7.3 \\
\hline 40 & 2 & 2 & $\mathrm{RF}$ & 15 & $\mathrm{n} 90$ & 1 & 118 & 15.47 & 51.40 & - & 4.20 & 2.028 & 978 & 3.8 & 539 & 4 \\
\hline 41 & 5 & 2 & $\mathrm{RF}$ & 15 & n90 & 1 & 118 & 15.48 & 50.84 & - & - & 1.874 & 856 & 4.3 & 517 & 7.3 \\
\hline 42 & 6 & 2 & $\mathrm{RF}$ & 15 & $\mathrm{n} 90$ & 1 & 116.5 & 15.35 & 51.96 & - & 4.323 & 2.049 & 1140 & 6.1 & 500 & 7.3 \\
\hline 43 & 7 & 2 & $\mathrm{RF}$ & 15 & n90 & 1 & - & - & - & - & - & - & - & - & 448 & 4.3 \\
\hline 44 & 8 & 2 & $\mathrm{RF}$ & 15 & $\mathrm{n} 90$ & 1 & 117.4 & 15.21 & 51.73 & - & 4.426 & 2.106 & 2265 & 9 & 2855 & 14.8 \\
\hline 45 & 0 & 2 & $\mathrm{RF}$ & 15 & 0 & 2 & 121 & 12.23 & 56.78 & 1843.9 & 3.153 & 1.434 & 1148 & 4.7 & 501 & 5.3 \\
\hline 46 & 2 & 2 & $\mathrm{RF}$ & 15 & 0 & 2 & 120.9 & 12.55 & 55.22 & - & 2.988 & 1.434 & 1122 & 2.8 & 361 & 3.1 \\
\hline 47 & 5 & 2 & $\mathrm{RF}$ & 15 & 0 & 2 & 122.6 & 11.72 & 56.62 & - & 3.099 & 1.447 & 1111 & 4.6 & 544 & 5.9 \\
\hline 48 & 6 & 2 & $\mathrm{RF}$ & 15 & 0 & 2 & 119.8 & 12.30 & 57.64 & - & 3.11 & 1.446 & 1241 & 5.5 & 550 & 5.9 \\
\hline 49 & 7 & 2 & $\mathrm{RF}$ & 15 & 0 & 2 & 120.3 & 12.55 & 54.71 & - & - & - & - & - & 244 & 2.8 \\
\hline 50 & 8 & 2 & $\mathrm{RF}$ & 15 & 0 & 2 & 122.8 & 13.80 & 44.76 & - & 3.407 & 1.383 & 3219 & 10.9 & 2599 & 12.5 \\
\hline 51 & 0 & 2 & $\mathrm{~S}$ & 15 & 0 & 1 & 126.4 & 8.54 & 75.8 & 1865.5 & 3.863 & 1.45 & 361 & 3.5 & 586 & 5.9 \\
\hline 52 & 2 & 2 & $\mathrm{~S}$ & 15 & 0 & 1 & 124.7 & 9.18 & 70.7 & - & 3.854 & 1.357 & 561 & 2.7 & 601 & 4 \\
\hline 53 & 5 & 2 & $S$ & 15 & 0 & 1 & 123.8 & 9.59 & 70.9 & - & 3.649 & 1.305 & 523 & 4 & 597 & 5.9 \\
\hline 54 & 6 & 2 & S & 15 & 0 & 1 & 125.1 & 8.22 & 77.51 & - & 3.871 & 1.438 & 359 & 3.9 & 585 & 5.9 \\
\hline 55 & 7 & 2 & $\mathrm{~S}$ & 15 & 0 & 1 & 126.3 & 9.09 & 71.46 & - & 3.919 & 1.368 & 363 & 2.1 & 472 & 3.5 \\
\hline 56 & 8 & 2 & $\mathrm{~S}$ & 15 & 0 & 1 & 134.5 & 5.27 & 78.93 & - & 4.008 & 1.714 & 321 & 4 & 2540 & 13.7 \\
\hline 57 & 0 & 2 & $\mathrm{~S}$ & 15 & 90 & 1 & 104.9 & 17.16 & 57.79 & 1844.1 & 4.133 & 1.422 & 2041 & 7.2 & 486 & 5.8 \\
\hline 58 & 2 & 2 & $\mathrm{~S}$ & 15 & 90 & 1 & 104.9 & 17.47 & 58.37 & - & 4.083 & 1.462 & 2144 & 5.4 & 376 & 3.7 \\
\hline 59 & 5 & 2 & S & 15 & 90 & 1 & 105.5 & 17.13 & 58.0 & - & - & 1.391 & 1905 & 8 & 489 & 5.8 \\
\hline 60 & 6 & 2 & $\mathrm{~S}$ & 15 & 90 & 1 & 103.7 & 17.31 & 58.32 & - & - & 1.458 & 2554 & 7.6 & 486 & 5.8 \\
\hline 61 & 7 & 2 & $\mathrm{~S}$ & 15 & 90 & 1 & 104.5 & 17.44 & 57.51 & - & 4.094 & 1.422 & 1291 & 4.5 & 182 & 2.7 \\
\hline 62 & 8 & 2 & $\mathrm{~S}$ & 15 & 90 & 1 & 104.7 & 16.79 & 57.94 & - & 4.264 & 1.449 & 4424 & 15.7 & 2617 & 14.2 \\
\hline 63 & 0 & 2 & $S$ & 15 & 0 & 2 & 134.4 & 8 & 71.04 & 1866.4 & 3.53 & 1.541 & 394 & 2.7 & 501 & 5.3 \\
\hline 64 & 2 & 2 & $\mathrm{~S}$ & 15 & 0 & 2 & 133.6 & 8.37 & 67.582 & - & 3.279 & 1.582 & 406 & 2.2 & 336 & 3.2 \\
\hline 65 & 5 & 2 & $\mathrm{~S}$ & 15 & 0 & 2 & 135.5 & 8.29 & 68.11 & - & 3.483 & 1.479 & 486 & 2.9 & 508 & 5.3 \\
\hline 66 & 6 & 2 & $\mathrm{~S}$ & 15 & 0 & 2 & 134.2 & 7.81 & 75.37 & - & 3.401 & 1.609 & 391 & 2.7 & 492 & 5.3 \\
\hline 67 & 7 & 2 & $\mathrm{~S}$ & 15 & 0 & 2 & 131.8 & 9.02 & 61.58 & - & 3.453 & 1.501 & 408 & 2.2 & 171 & 3.3 \\
\hline 68 & 8 & 2 & $\mathrm{~S}$ & 15 & 0 & 2 & 132.7 & 8.94 & 70.26 & - & 3.855 & 1.35 & 1726 & 8.5 & 2542 & 12.2 \\
\hline 69 & 0 & 2 & $\mathrm{~S}$ & 50 & 0 & 1 & 143.9 & 5.59 & 84.1 & 1884.5 & 2.311 & 1.564 & 333 & 1.9 & 566 & 6.8 \\
\hline 70 & 2 & 2 & $S$ & 50 & 0 & 1 & 148.8 & 3.58 & 87.65 & - & 1.873 & 1.608 & 350 & 1.1 & 375 & 3.8 \\
\hline 71 & 5 & 2 & $\mathrm{~S}$ & 50 & 0 & 1 & 146.7 & 4.27 & 77.80 & - & 2.615 & 1.584 & 276 & 1.4 & 569 & 6.8 \\
\hline 72 & 6 & 2 & $\mathrm{~S}$ & 50 & 0 & 1 & 141.0 & 6.68 & 70.23 & - & 2.111 & 1.565 & 419 & 1.9 & 565 & 6.8 \\
\hline 73 & 7 & 2 & $\mathrm{~S}$ & 50 & 0 & 1 & 140.2 & 6.02 & 82.35 & - & 2.728 & 1.603 & 305 & 2.3 & 237 & 3.7 \\
\hline 74 & 8 & 2 & $\mathrm{~S}$ & 50 & 0 & 1 & 141.4 & 6.15 & 87.22 & - & 2.969 & 1.572 & 740 & 5.3 & 2562 & 13.7 \\
\hline 75 & 0 & 1 & LF & 50 & 0 & 2 & 143.3 & 10.799 & 59.81 & 2076.6 & 3.697 & 1.415 & 1720 & 7.3 & 1608 & 7 \\
\hline 76 & 1 & 1 & LF & 50 & 0 & 2 & - & - & - & - & 3.635 & 1.412 & 1576 & 7.7 & 1385 & 7.1 \\
\hline 77 & 0 & 1 & S & 50 & 0 & 2 & 144.2 & 8.973 & 65.04 & 2028.1 & 3.749 & 1.548 & 1327 & 6.2 & 1740 & 8.4 \\
\hline
\end{tabular}




\begin{tabular}{|c|c|c|c|c|c|c|c|c|c|c|c|c|c|c|c|c|}
\hline 78 & 1 & 1 & $\mathrm{~S}$ & 50 & 0 & 2 & - & - & - & - & 3.676 & 1.565 & 1158 & 6.6 & 1740 & 7.6 \\
\hline 79 & 0 & 1 & $\mathrm{~S}$ & 50 & 90 & 2 & 126 & 17.07 & 48.48 & 2084.8 & 3.912 & 1.465 & 3239 & 7.2 & 1298 & 5.7 \\
\hline 80 & 1 & 1 & $\mathrm{~S}$ & 50 & 90 & 2 & - & - & - & - & 3.847 & 1.471 & 3006 & 6.8 & 1157 & 5.7 \\
\hline 81 & 0 & 1 & $\mathrm{LF}$ & 85 & 0 & 1 & 143.4 & 11.422 & 62.28 & 2107.7 & 4.206 & 1.203 & 1640 & 6.9 & 1507 & 7.1 \\
\hline 82 & 1 & 1 & $\mathrm{LF}$ & 85 & 0 & 1 & - & - & - & - & 4.096 & 1.202 & 1482 & 7.7 & 1077 & 6.4 \\
\hline 83 & 0 & 1 & $\mathrm{LF}$ & 85 & 0 & 2 & 150.6 & 11.415 & 54.72 & 2175.6 & 4.54 & 1.372 & 964 & 4.9 & 954 & 5.2 \\
\hline 84 & 1 & 1 & $\mathrm{LF}$ & 85 & 0 & 2 & - & - & - & - & 4.442 & 1.373 & 792 & 4.4 & 834 & 4.8 \\
\hline 85 & 0 & 1 & $\mathrm{LF}$ & 85 & 90 & 2 & 151.5 & 16.048 & 47.27 & 2152.2 & 4.77 & 1.317 & 1890 & 6.3 & 906 & 4.6 \\
\hline 86 & 1 & 1 & $\mathrm{LF}$ & 85 & 90 & 2 & - & - & - & - & 4.711 & 1.327 & 1650 & 6 & 754 & 4.1 \\
\hline 87 & 0 & 1 & $\mathrm{RF}$ & 85 & 0 & 1 & 140 & 12.423 & 55.57 & 2135.1 & 4.197 & 1.165 & 1392 & 6.2 & 1218 & 5.8 \\
\hline 88 & 1 & 1 & $\mathrm{RF}$ & 85 & 0 & 1 & - & - & - & - & 4.10 & 1.172 & 1356 & 6.5 & 1077 & 5.7 \\
\hline 89 & 0 & 1 & $\mathrm{RF}$ & 85 & 0 & 2 & 142.3 & 12.456 & 52.76 & 2113.2 & 4.132 & 1.235 & 1362 & 5.6 & 1113 & 5.1 \\
\hline 90 & 1 & 1 & $\mathrm{RF}$ & 85 & 0 & 2 & - & - & - & - & 3.949 & 1.228 & 1247 & 5.5 & 951 & 4.8 \\
\hline 91 & 0 & 1 & $\mathrm{~S}$ & 85 & 0 & 1 & 149.8 & 8.07 & 86.05 & 2043.2 & 4.716 & 1.427 & 1551 & 8.5 & 2196 & 9.8 \\
\hline 92 & 1 & 1 & $\mathrm{~S}$ & 85 & 0 & 1 & - & - & - & - & 4.671 & 1.441 & 1359 & 7.7 & 1986 & 9.2 \\
\hline 93 & 0 & 1 & $\mathrm{~S}$ & 85 & 0 & 2 & 149.9 & 10.35 & 55.25 & 2151 & 4.748 & 1.452 & 784 & 4.5 & 926 & 4.7 \\
\hline 94 & 1 & 1 & $\mathrm{~S}$ & 85 & 0 & 2 & - & - & - & - & 4.777 & 1.418 & 650 & 3.9 & 782 & 4.2 \\
\hline 95 & 0 & 1 & $\mathrm{~S}$ & 85 & 90 & 2 & 133.4 & 17.03 & 45.81 & 2170.6 & 4.844 & 1.255 & 2155 & 6.7 & 865 & 4.8 \\
\hline 96 & 1 & 1 & $\mathrm{~S}$ & 85 & 90 & 2 & - & - & - & - & 4.755 & 1.253 & 2096 & 6.9 & 711 & 4.2 \\
\hline 97 & 0 & 1 & $\mathrm{~S}$ & 85 & n90 & 2 & 129 & 16.553 & 45.67 & 2148.7 & 5.260 & 1.929 & 1713 & 6.6 & 932 & 4.8 \\
\hline 98 & 1 & 1 & $\mathrm{~S}$ & 85 & n90 & 2 & - & - & - & - & 5.09 & 1.901 & 1670 & 6.3 & 788 & 4.2 \\
\hline 99 & 0 & 1 & $\mathrm{LF}$ & 50 & 90 & 2 & 141.8 & 15.79 & 51.22 & 2031.7 & 4.016 & 1.551 & 3391 & 9.5 & 1714 & 8.4 \\
\hline 100 & 0 & 1 & $\mathrm{LF}$ & 50 & n90 & 2 & 133.8 & 16.61 & 45.96 & 2012.5 & 4.247 & 1.934 & 3194 & 10 & 2159 & 8.5 \\
\hline 101 & 0 & 1 & $\mathrm{RF}$ & 50 & 0 & 2 & 133.3 & 11.83 & 58.04 & 2011.5 & 3.522 & 1.357 & 3540 & 10.5 & 2308 & 8.5 \\
\hline 102 & 0 & 1 & $\mathrm{RF}$ & 50 & 90 & 2 & 139.7 & 16.557 & 52.01 & 2022.7 & 4.006 & 1.478 & 4536 & 10.7 & 2087 & 8.4 \\
\hline 103 & 0 & 1 & $\mathrm{~S}$ & 50 & n90 & 2 & 120.9 & 16.993 & 47.93 & 2055.7 & 4.305 & 2.239 & 2914 & 10 & 1627 & 7.2 \\
\hline 104 & 0 & 1 & LF & 85 & 90 & 1 & 142.7 & 16.346 & 58.9 & 2067.9 & 4.758 & 1.352 & 3549 & 12.7 & 2385 & 8.5 \\
\hline 105 & 0 & 1 & LF & 85 & n90 & 2 & 143.7 & 16.434 & 46.07 & 2077.2 & 5.248 & 1.804 & 2028 & 8.3 & 1549 & 6.3 \\
\hline 106 & 0 & 1 & $\mathrm{RF}$ & 85 & 90 & 1 & 142.5 & 16.41 & 59.58 & 2063.7 & 4.764 & 1.373 & 3486 & 13 & 2486 & 8.6 \\
\hline 107 & 0 & 1 & RF & 85 & 90 & 2 & 150.3 & 16.48 & 47.64 & 2143.8 & 4.80 & 1.307 & 2146 & 7.1 & 953 & 4.8 \\
\hline 108 & 0 & 1 & $\mathrm{RF}$ & 85 & n90 & 2 & 144.6 & 16.05 & 47.65 & 2060.3 & 5.158 & 1.744 & 1947 & 9.2 & 1506 & 6.6 \\
\hline 109 & 0 & 1 & $\mathrm{~S}$ & 85 & 90 & 1 & 124.2 & 17.64 & 55.9 & 2096.0 & 4.748 & 1.233 & 3973 & 11.7 & 1699 & 7.7 \\
\hline 110 & 0 & 1 & $\mathrm{~S}$ & 85 & n90 & 1 & 120.4 & 16.92 & 50.93 & 2067.4 & - & 1.876 & 3135 & 13.8 & 2172 & 8.2 \\
\hline 111 & 0 & 3 & $\mathrm{LF}$ & 15 & 0 & 2 & 133.2 & 10.17 & 66.09 & 1869.1 & - & 1.519 & 819 & 3.6 & - & - \\
\hline 112 & 0 & 3 & $\mathrm{LF}$ & 15 & 90 & 2 & 129.9 & 15.86 & 51.3 & 1936.8 & - & 1.729 & 5340 & 11.4 & - & - \\
\hline 113 & 0 & 3 & LF & 15 & n90 & 2 & 122.5 & 16.53 & 46.45 & 1956.7 & - & 2.057 & 2864 & 9.7 & - & - \\
\hline 114 & 0 & 3 & $\mathrm{RF}$ & 15 & 90 & 2 & 129.1 & 16.27 & 52.5 & 1946.7 & - & 1.59 & 5283 & 11 & - & - \\
\hline 115 & 0 & 3 & $\mathrm{RF}$ & 15 & n90 & 2 & 124.9 & 16.09 & 47.06 & 1955.4 & - & 2.452 & 3090 & 9.4 & - & - \\
\hline 116 & 0 & 3 & $\mathrm{~S}$ & 15 & n90 & 1 & 99.9 & 17.21 & 50.12 & 1864.5 & - & 1.686 & 1129 & 6.1 & - & - \\
\hline 117 & 0 & 3 & $\mathrm{~S}$ & 15 & 90 & 2 & 113.5 & 16.97 & 51.72 & 1947.4 & - & 1.616 & 5147 & 10.9 & - & - \\
\hline 118 & 0 & 3 & $\mathrm{~S}$ & 15 & n90 & 2 & 107.5 & 17.10 & 47.97 & 1947.0 & - & 2.080 & 4244 & 10.9 & - & - \\
\hline 119 & 0 & 3 & $\mathrm{LF}$ & 50 & 0 & 1 & 130.3 & 11.45 & 63.19 & 1920.6 & - & 1.369 & 1688 & 7.6 & - & - \\
\hline 120 & 0 & 3 & $\mathrm{LF}$ & 50 & 90 & 1 & 130.7 & 16.35 & 59.46 & 2002.3 & - & 1.434 & 3824 & 10 & - & - \\
\hline
\end{tabular}




\begin{tabular}{|c|c|c|c|c|c|c|c|c|c|c|c|c|c|c|c|c|}
\hline 121 & 0 & 3 & LF & 50 & n90 & 1 & 127.8 & 15.95 & 52.78 & 1990.7 & - & 1.947 & 2919 & 10 & - & - \\
\hline 122 & 0 & 3 & $\mathrm{RF}$ & 50 & 0 & 1 & 126.5 & 12.79 & 61.04 & 1935.1 & - & 1.31 & 3515 & 10.2 & - & - \\
\hline 123 & 0 & 3 & $\mathrm{RF}$ & 50 & 90 & 1 & 130.7 & 16.51 & 59.6 & 1991.4 & - & 1.45 & 3782 & 10.7 & - & - \\
\hline 124 & 0 & 3 & $\mathrm{RF}$ & 50 & n90 & 1 & 127.7 & 15.96 & 53.06 & 1985.5 & - & 1.983 & 2940 & 9.8 & - & - \\
\hline 125 & 0 & 3 & $\mathrm{RF}$ & 50 & n90 & 2 & 134.4 & 16.39 & 47.14 & 1970.2 & - & 1.694 & 3911 & 11.2 & - & - \\
\hline 126 & 0 & 3 & S & 50 & 90 & 1 & 116.1 & 17.81 & 58.51 & 2030.5 & - & 1.436 & 5696 & 13.3 & - & - \\
\hline 127 & 0 & 3 & S & 50 & n90 & 1 & 108.9 & 17.57 & 50.99 & 2000.3 & - & 1.629 & 4013 & 10.3 & - & - \\
\hline 128 & 0 & 3 & LF & 85 & n90 & 1 & 138.3 & 15.85 & 51.98 & 2010.2 & - & 1.352 & 2751 & 10 & - & - \\
\hline 129 & 0 & 3 & $\mathrm{RF}$ & 85 & n90 & 1 & 138.2 & 15.78 & 51.61 & 2034.7 & - & 1.557 & 2794 & 10.2 & - & - \\
\hline
\end{tabular}

* Configuration 1: Head impacting windshield while torso only deforming the hood (The head impact timing, speed, and angle of vehicle design 1should be the same with vehicle design 0, and thus indicated as “-”)

* Configuration 2: Head impacting hood after torso deforming the hood

* Configuration 3: Head impacting both hood and windshield

* Posture: LF - left leg forward; RF - right leg forward; S - standing

* Anthropometry: $15-15^{\text {th }}$ male; $50-50^{\text {th }}$ male; $85-85^{\text {th }}$ male;

* Direction: 0 - pure lateral; 90 - facing away; n90 - facing vehicle

* Location: 1 - center; 2 - corner

* Head impact timing: ms

* Head impact speed: $\mathrm{m} / \mathrm{s}$

* Head impact angle: ${ }^{\circ}$

* Head impact mass: kg

* Wrap around distance: mm (the difference of head impact locations caused by vehicle design was verified to be ignorable and thus their WADs were indicated as “-”)

* - : Results not calculated or model running failed

\subsubsection{Time History Output of Baseline Models for Hypothesis Testing}

\section{Angular velocity}
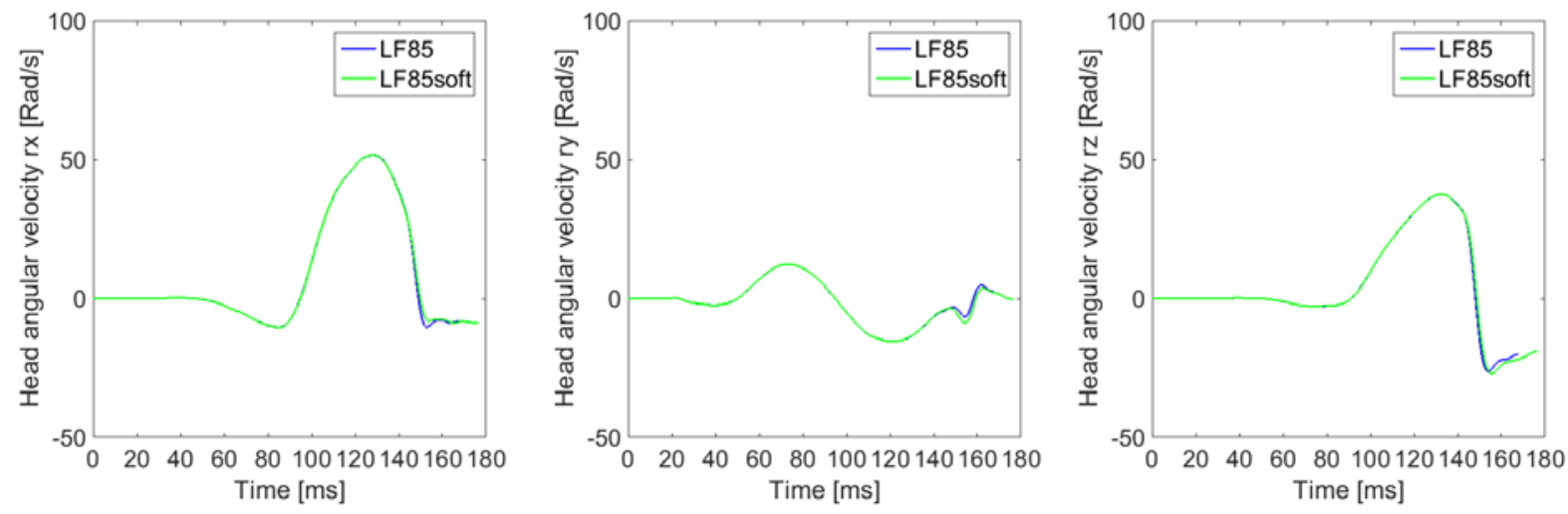

Figure 4.20 Time history of angular velocity of LF85 (Left - rx; Middle - ry; Right - rz) 

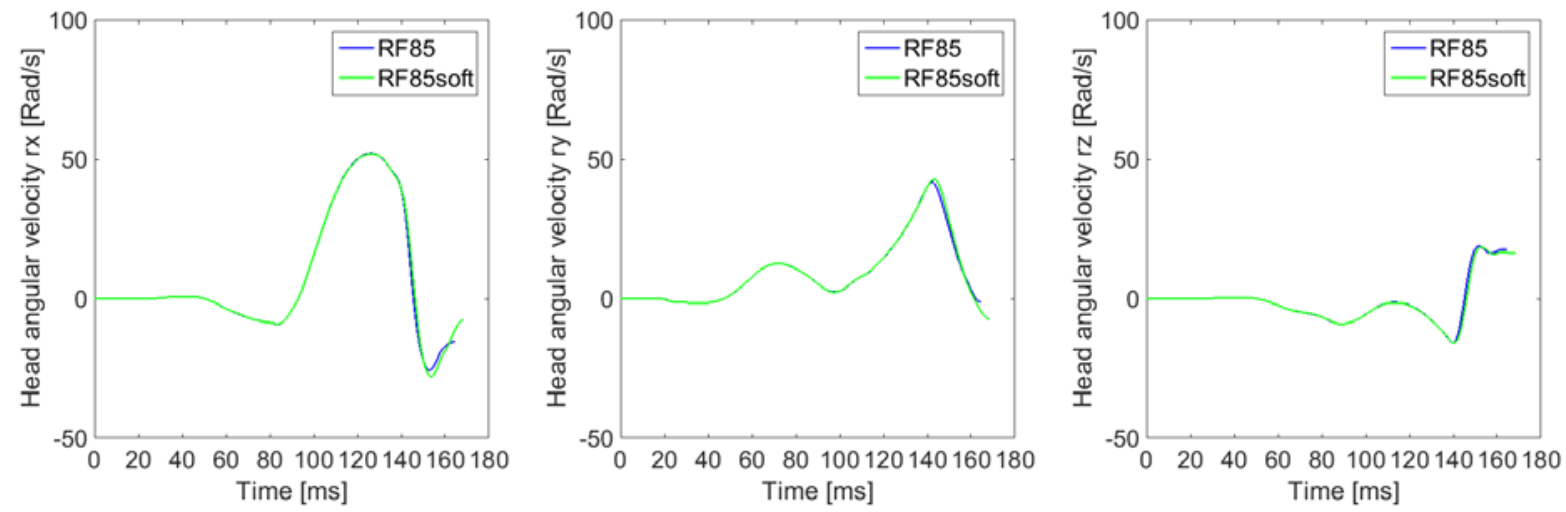

Figure 4.21 Time history of angular velocity of RF85 (Left - rx; Middle - ry; Right - rz)
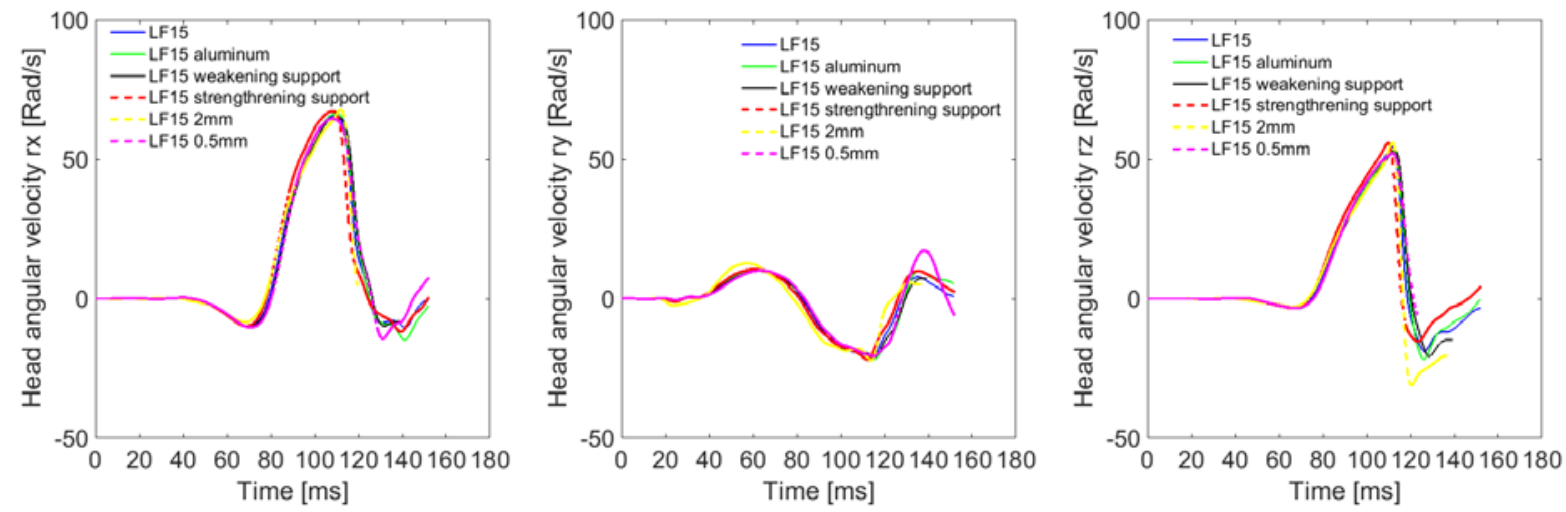

Figure 4.22 Time history of angular velocity of LF15 (Left - rx; Middle - ry; Right - rz)
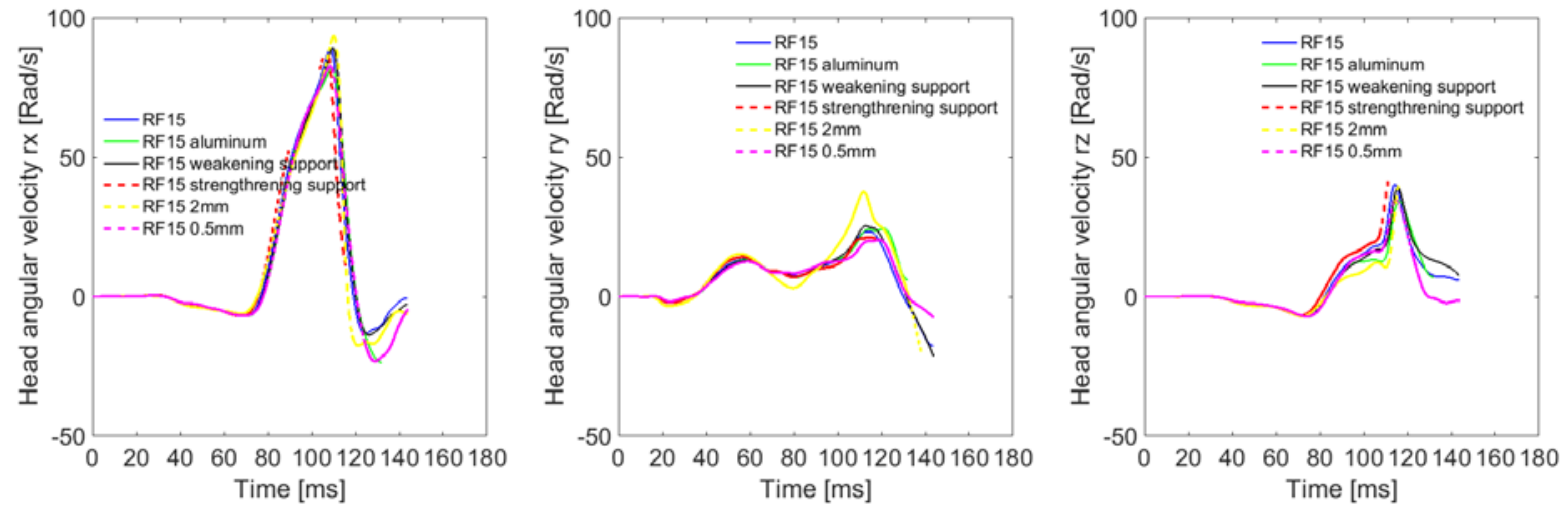

Figure 4.23 Time history of angular velocity of RF15 (Left - rx; Middle - ry; Right - rz) 


\section{$\underline{\text { Impact configuration } 1}$}

* In all the following graphs, the starting time of time history (time $=0 \mathrm{~ms}$ ) is the head impact just happening.
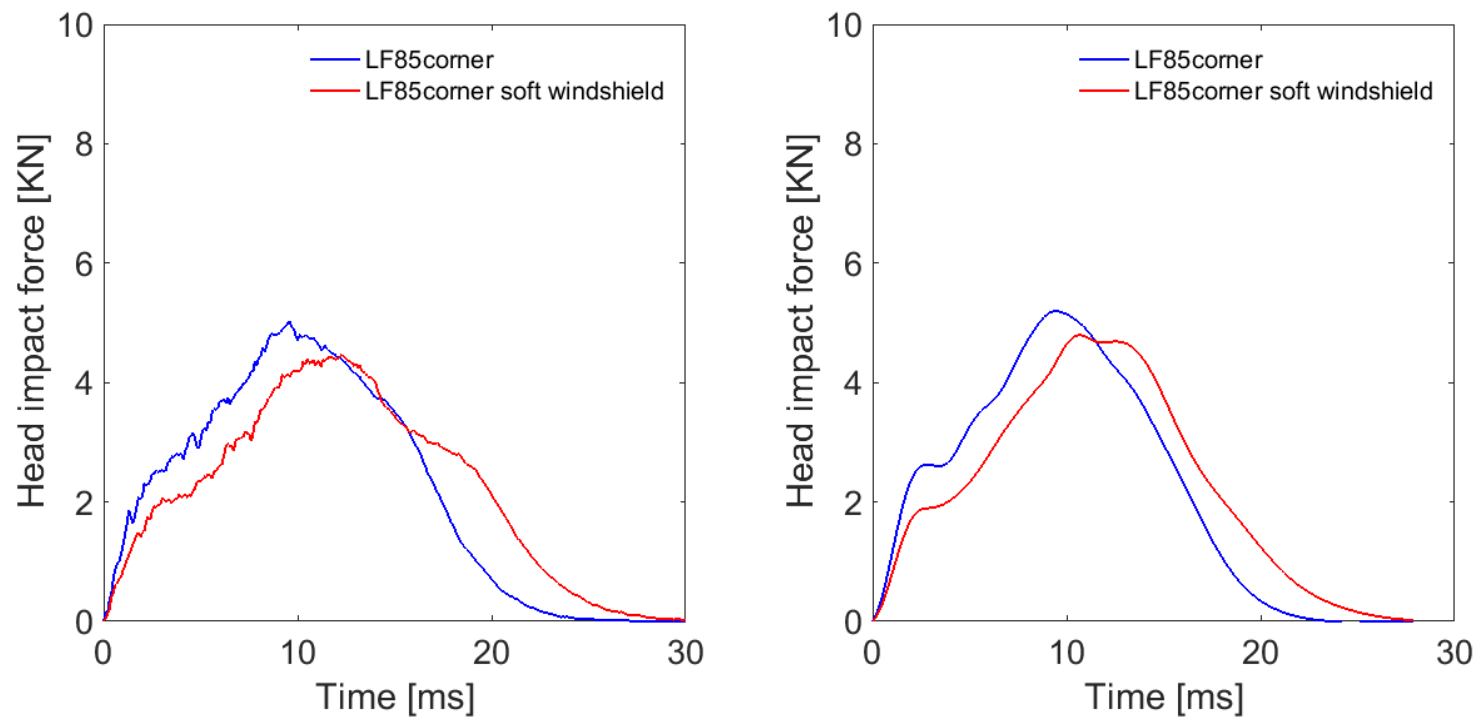

Figure 4.24 Time history of head impact force of LF85corner (Left - full-scale impact; Right - impactor test)
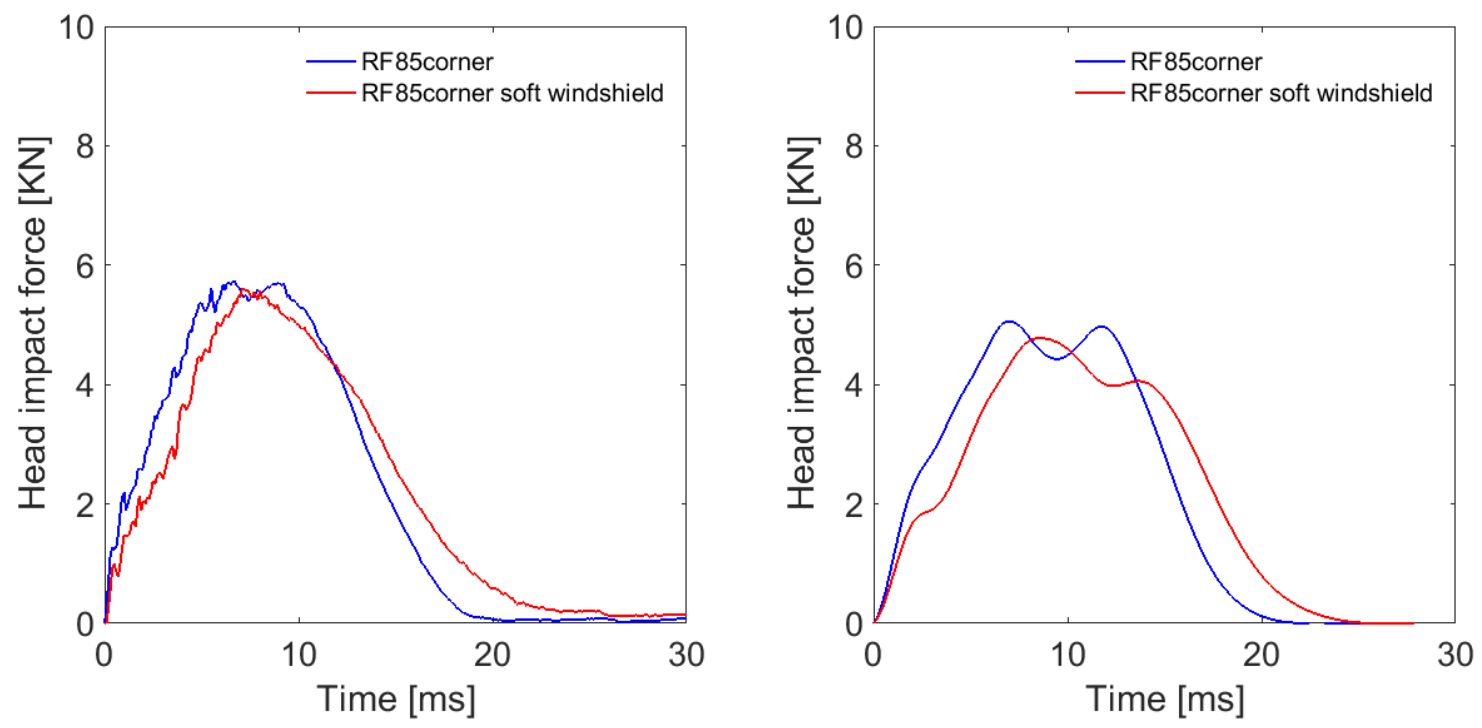

Figure 4.25 Time history of head impact force of RF85corner (Left - full-scale impact; Right - impactor test) 

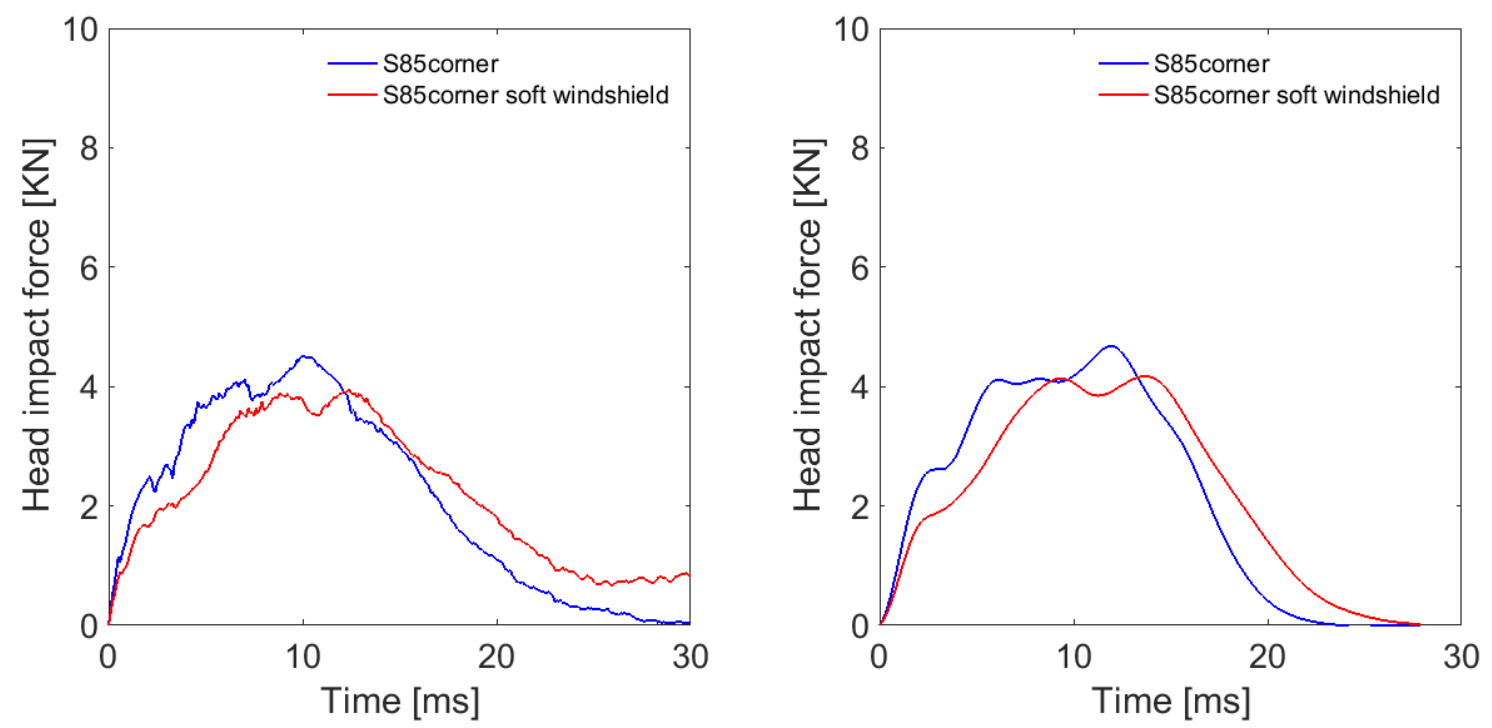

Figure 4.26 Time history of head impact force of S85corner (Left - full-scale impact; Right - impactor test)
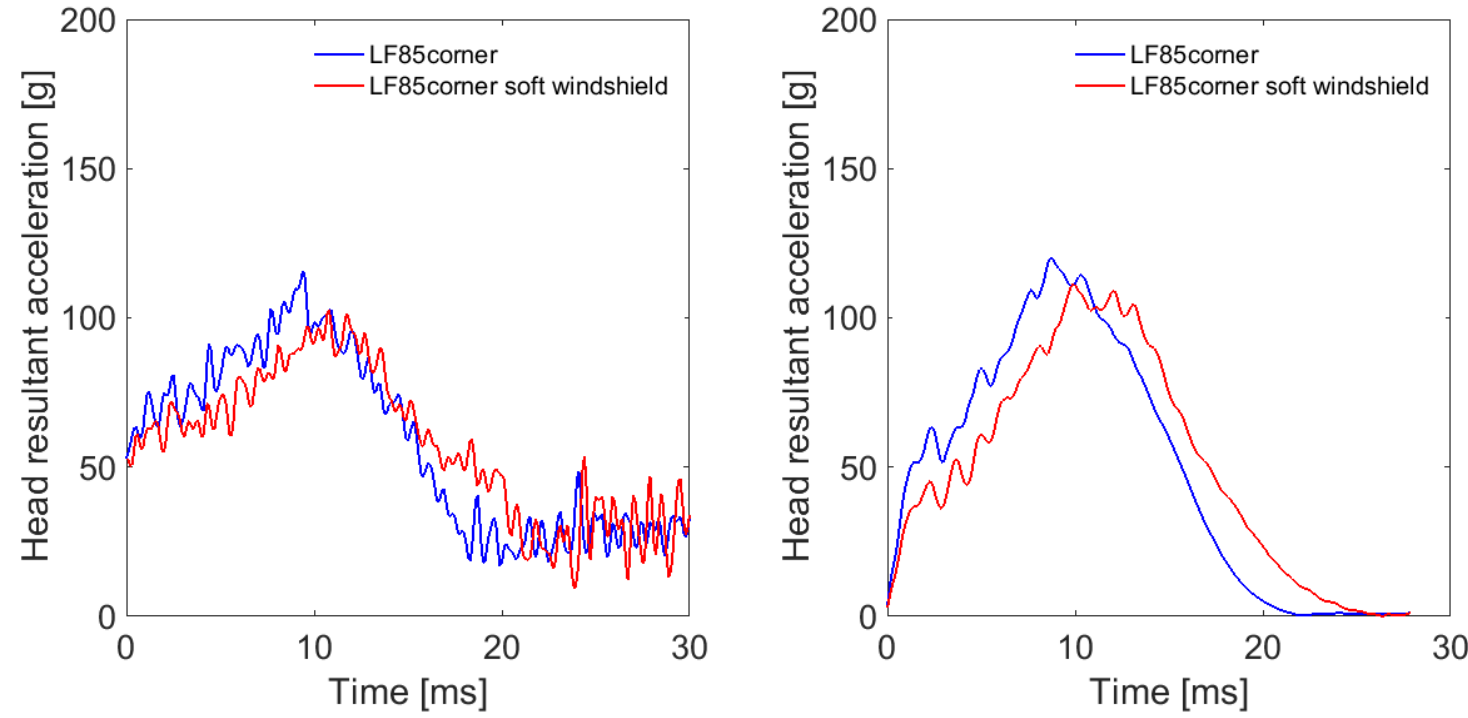

Figure 4.27 Time history of resultant head acceleration of LF85corner (Left - full-scale impact; Right impactor test) 

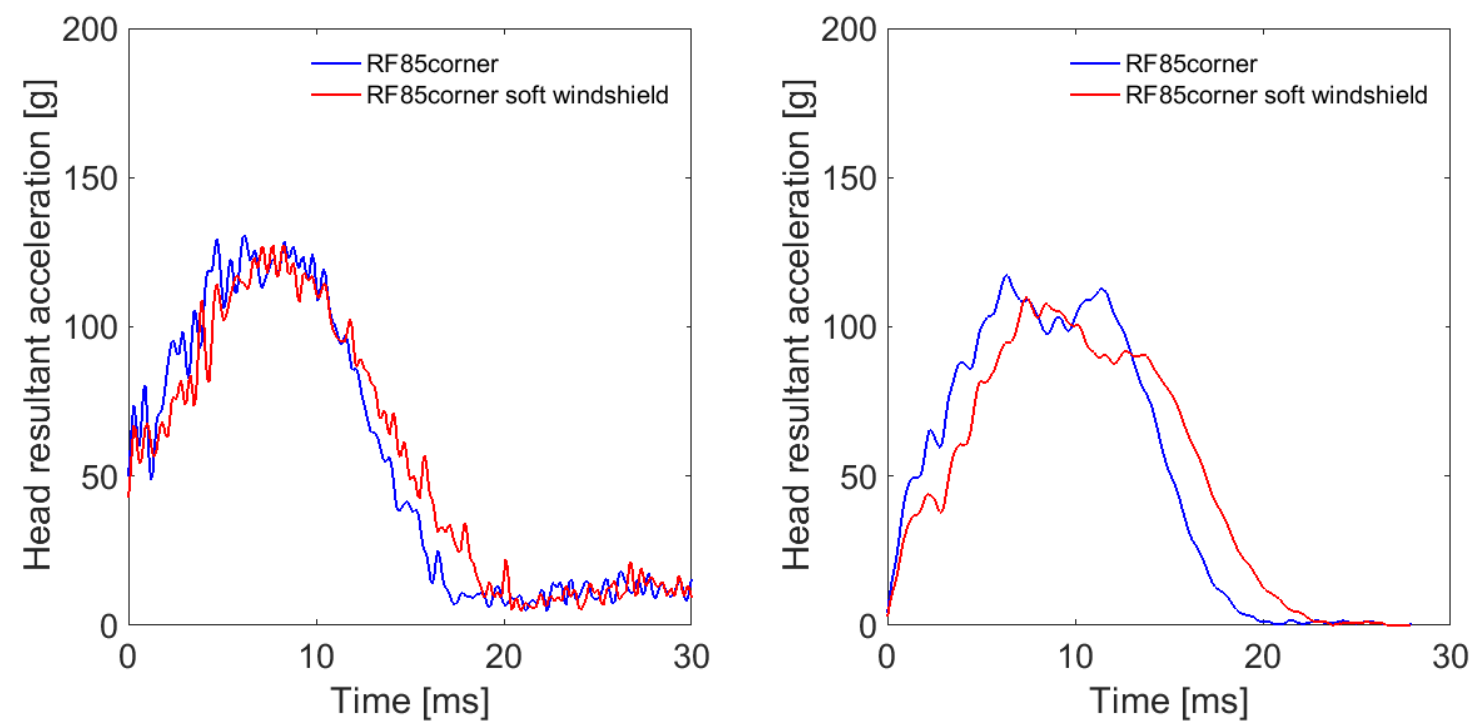

Figure 4.28 Time history of resultant head acceleration of RF85corner (Left - full-scale impact; Right impactor test)
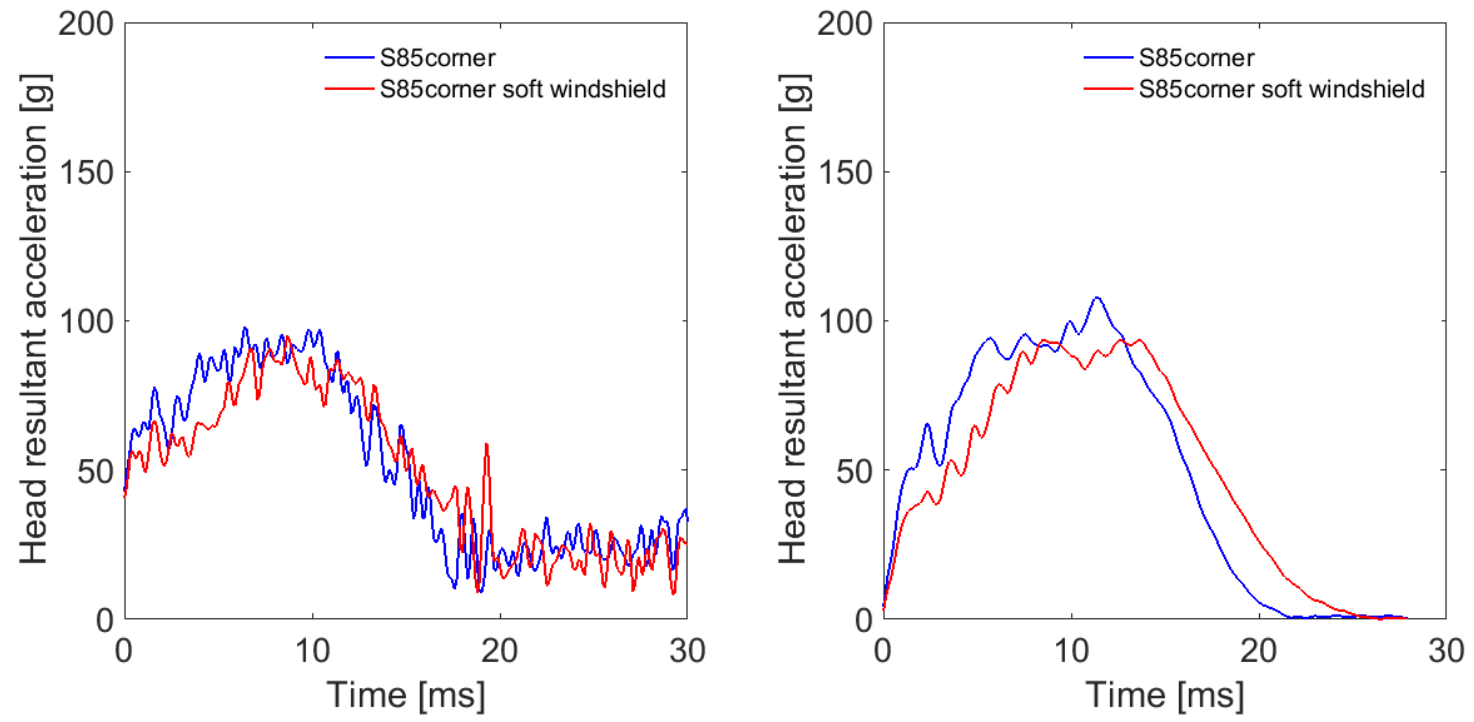

Figure 4.29 Time history of resultant head acceleration of S85corner (Left - full-scale impact; Right impactor test) 


\section{Impact configuration 2}
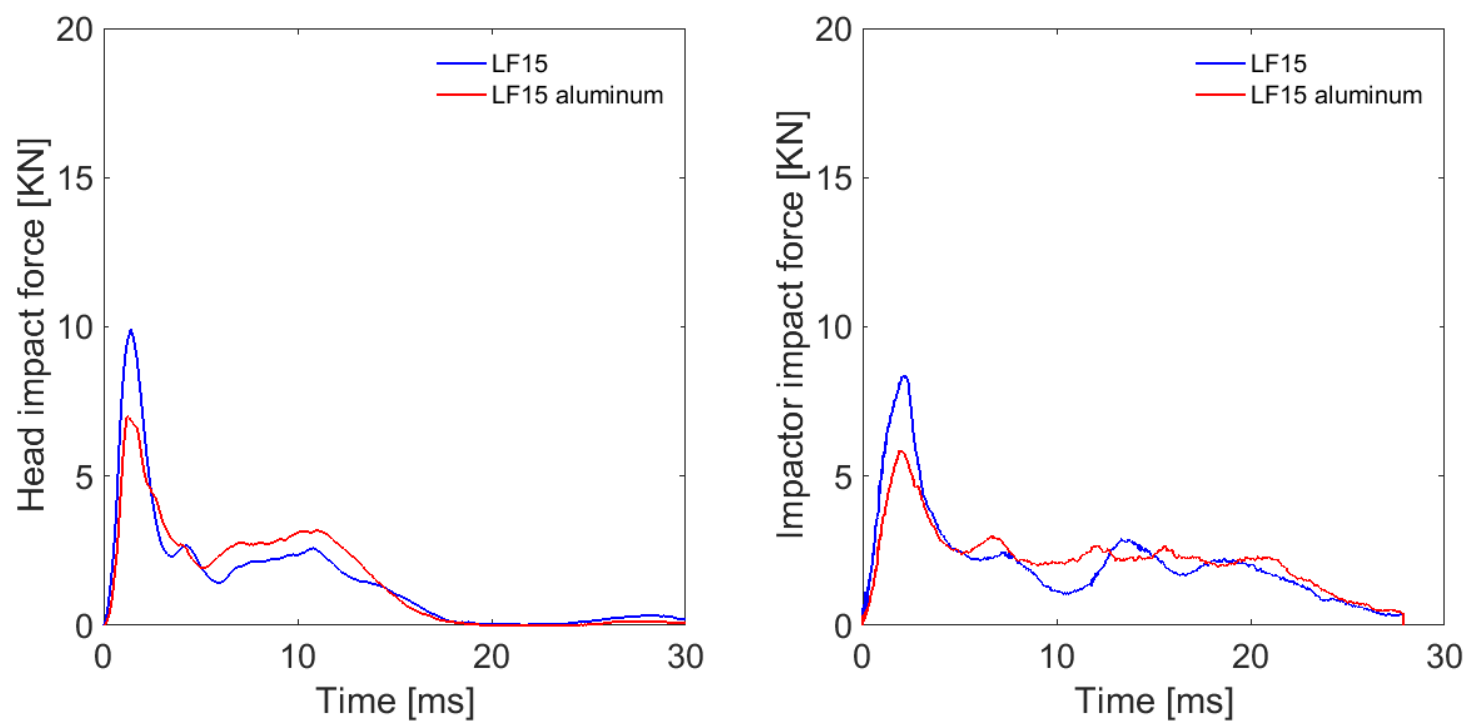

Figure 4.30 Time history of head impact force when varying material stiffness of LF15 (Left - full-scale impact; Right - impactor test)
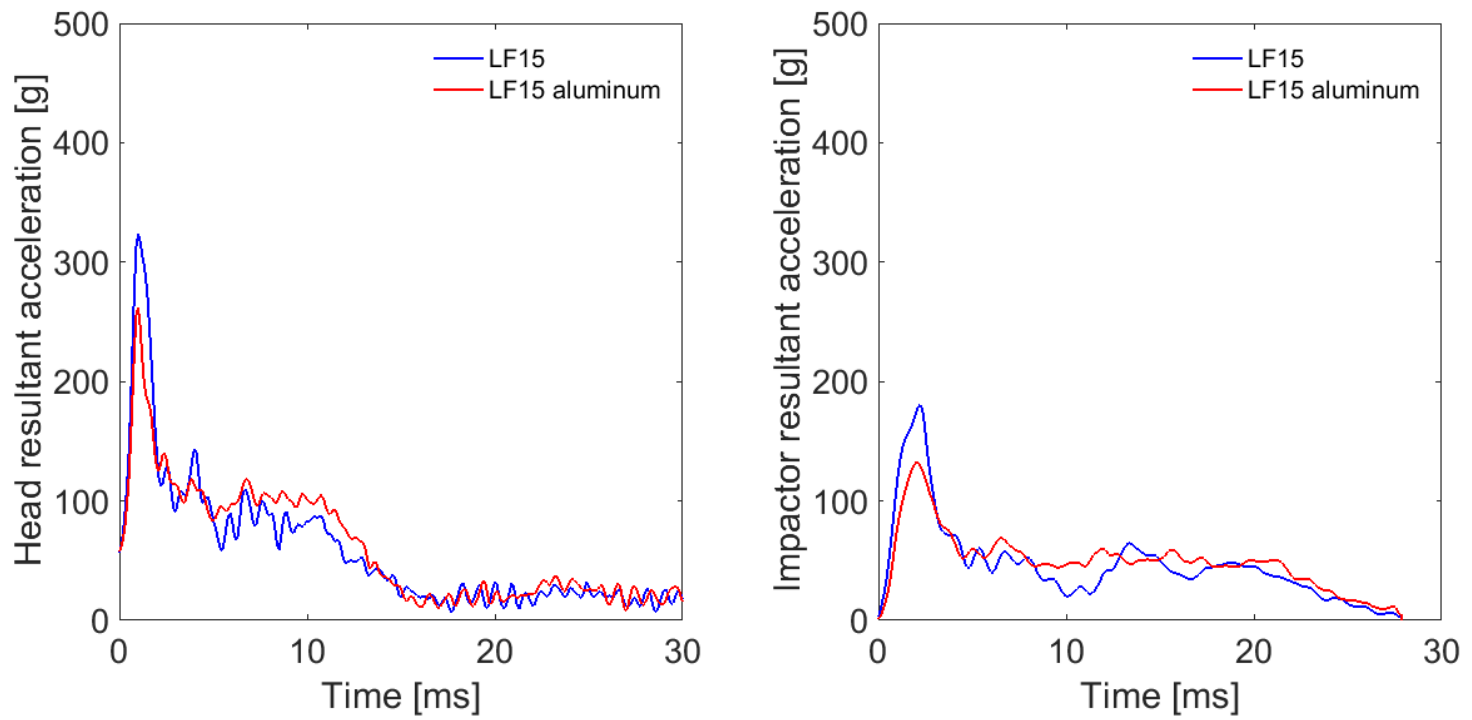

Figure 4.31 Time history of head acceleration when varying material stiffness of LF15 (Left - full-scale impact; Right - impactor test) 

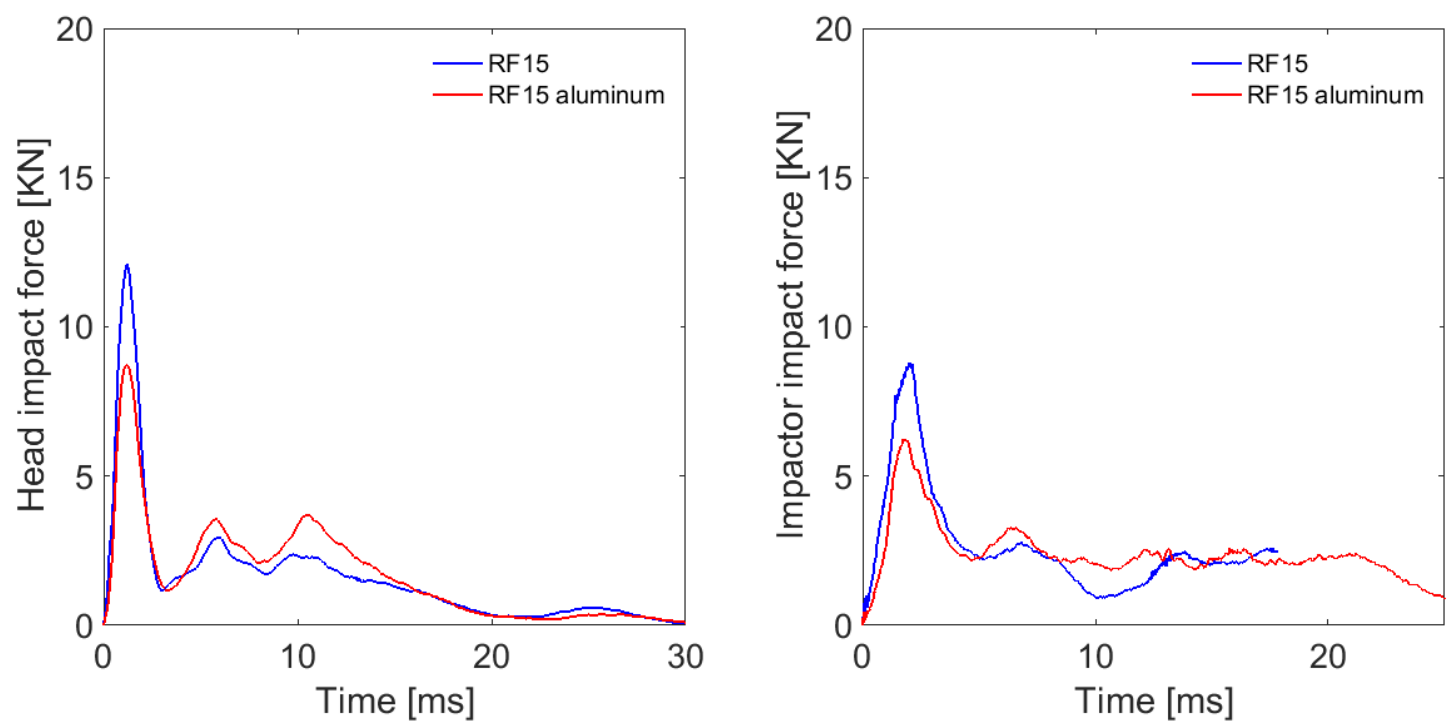

Figure 4.32 Time history of head impact force when varying material stiffness of RF15 (Left - full-scale impact; Right - impactor test)
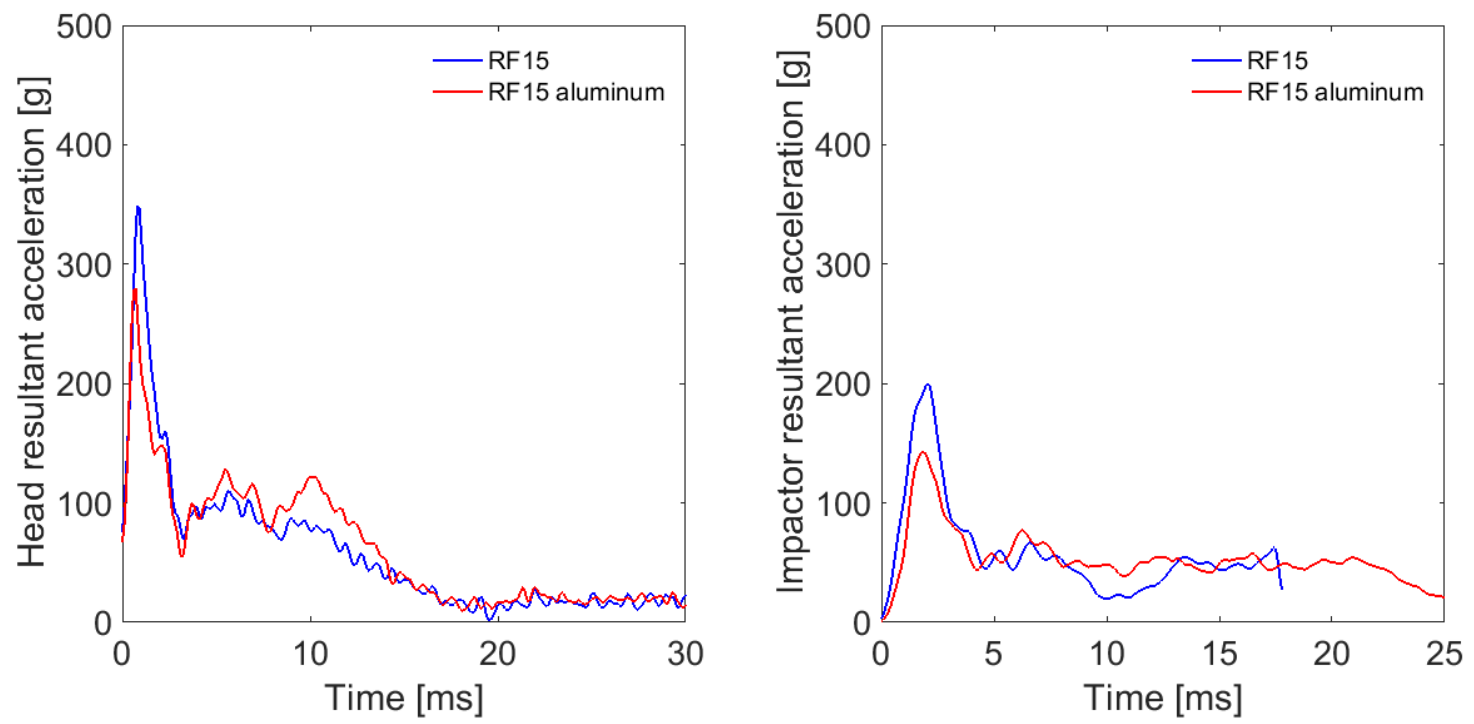

Figure 4.33 Time history of head acceleration when varying material stiffness of RF15 (Left - full-scale impact; Right - impactor test) 

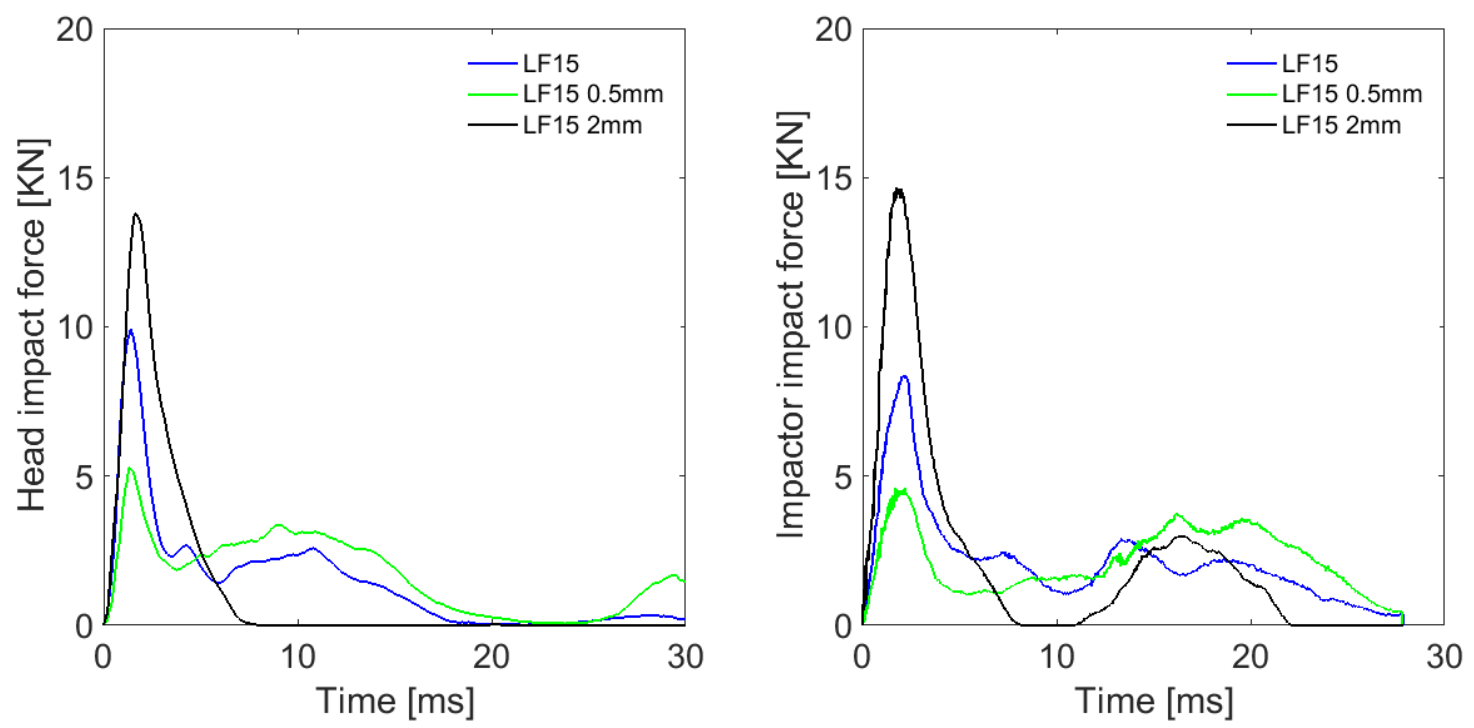

Figure 4.34 Time history of head impact force when varying hood thickness of LF15 (Left - full-scale impact; Right - impactor test)
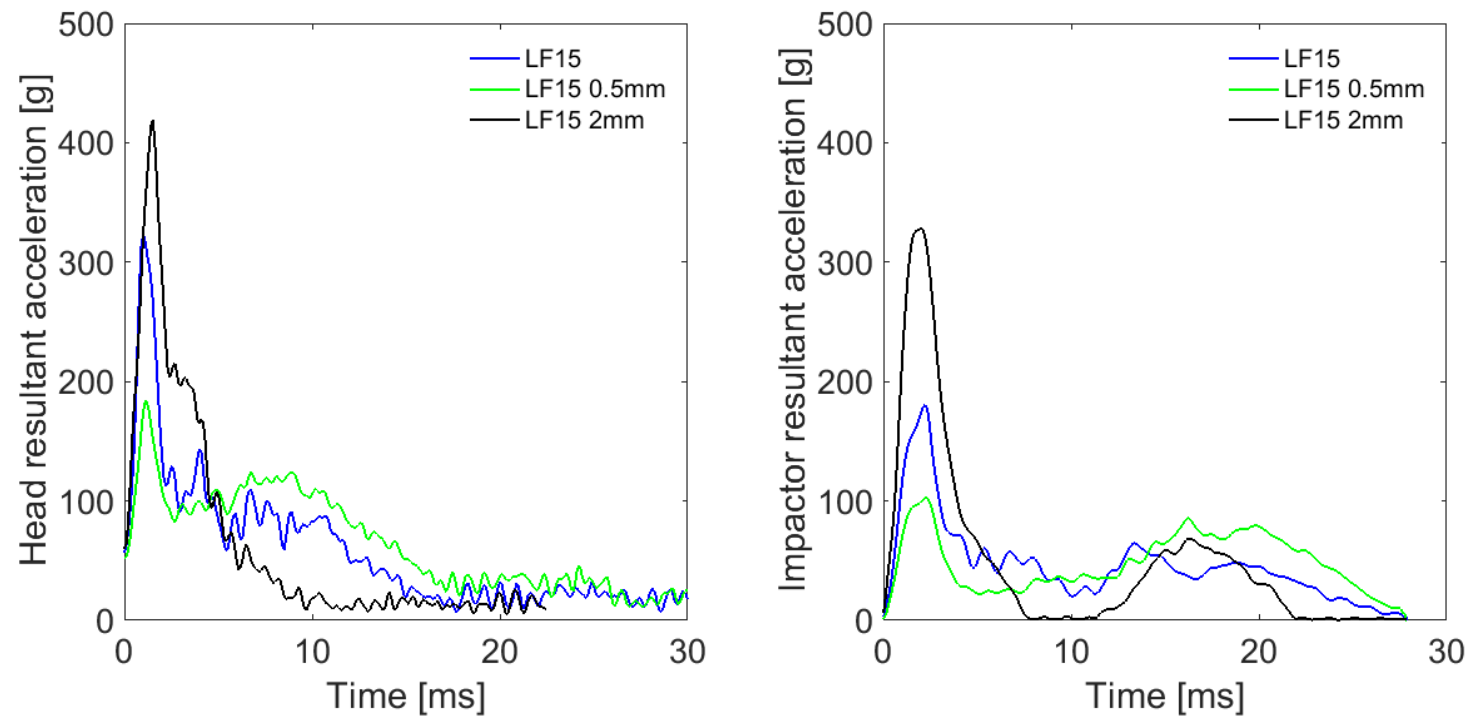

Figure 4.35 Time history of head acceleration when varying hood thickness of LF15 (Left - full-scale impact; Right - impactor test) 

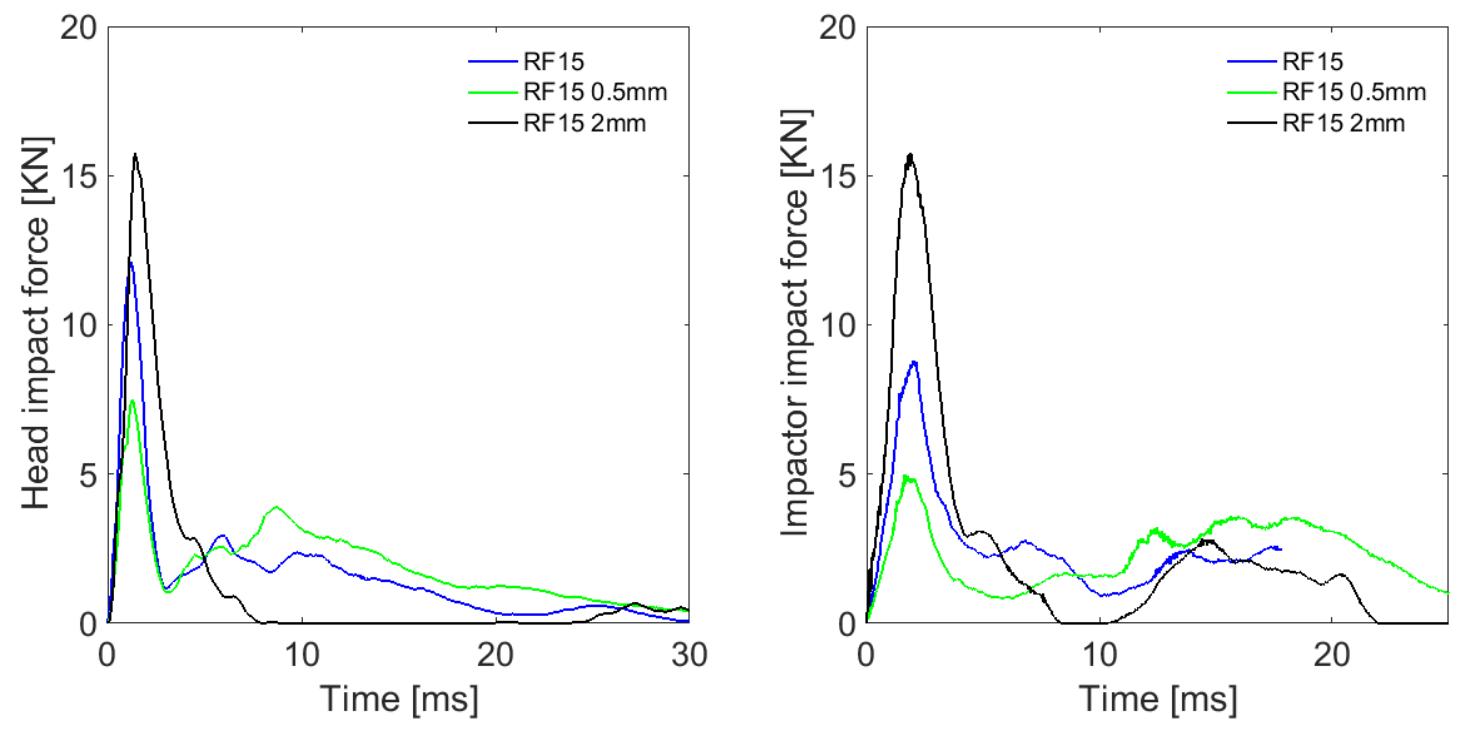

Figure 4.36 Time history of head impact force when varying hood thickness of RF15 (Left - full-scale impact; Right - impactor test)
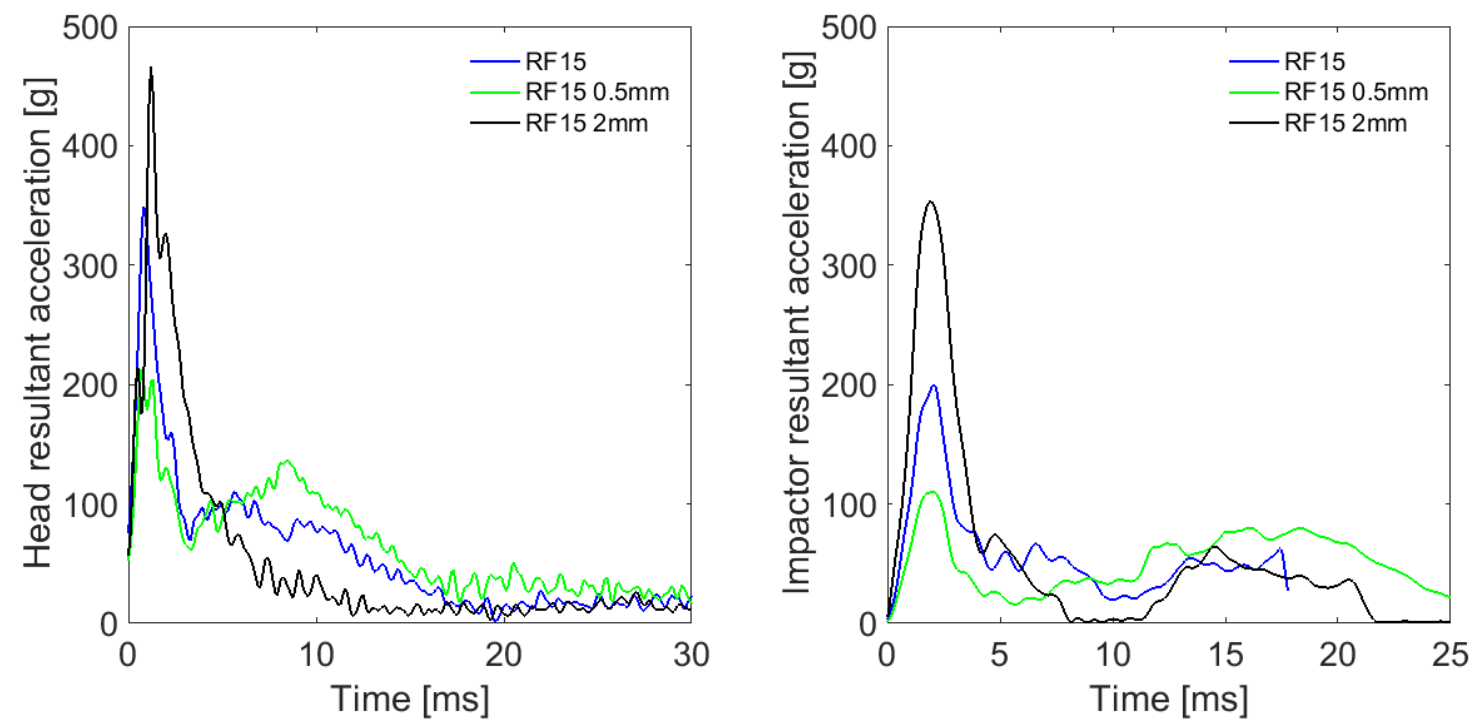

Figure 4.37 Time history of head acceleration when varying hood thickness of RF15 (Left - full-scale impact; Right - impactor test) 

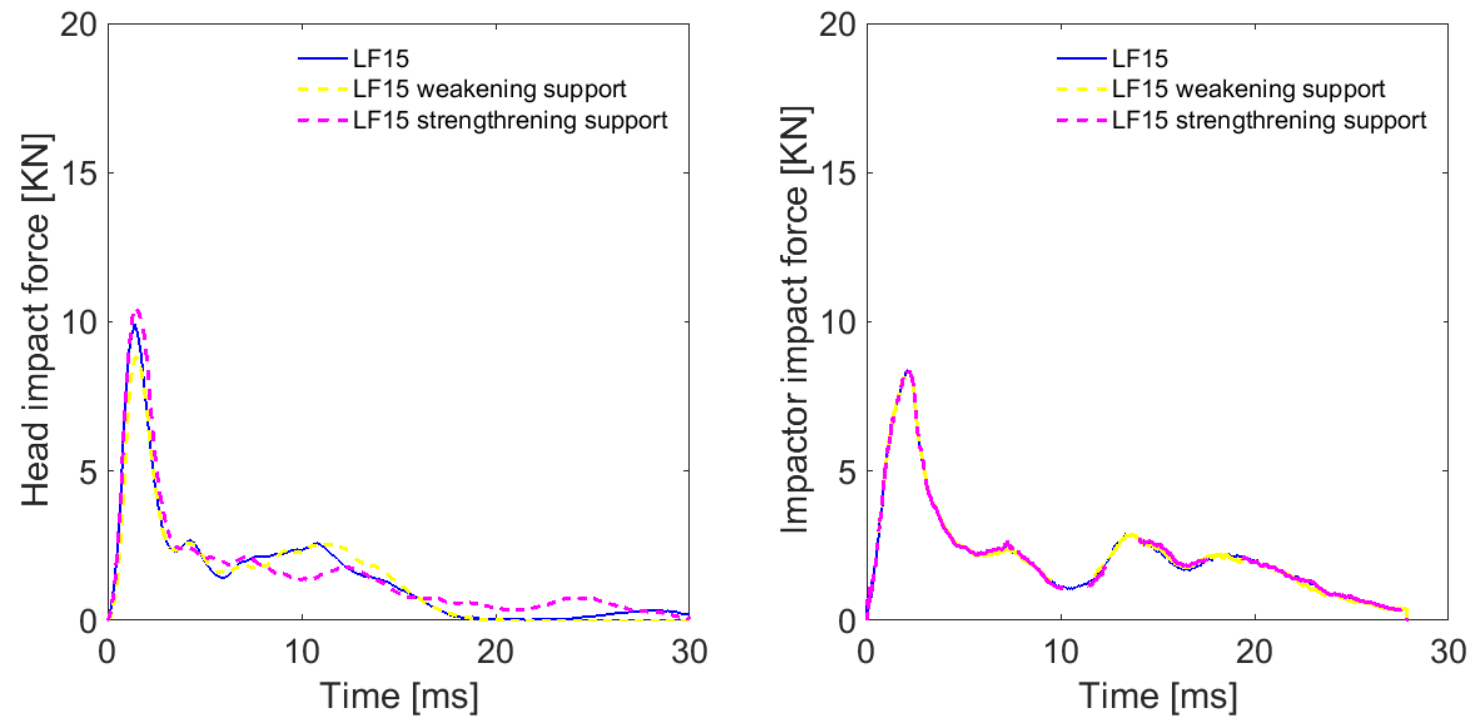

Figure 4.38 Time history of head impact force when varying hood hinge support of LF15 (Left - full-scale impact; Right - impactor test)
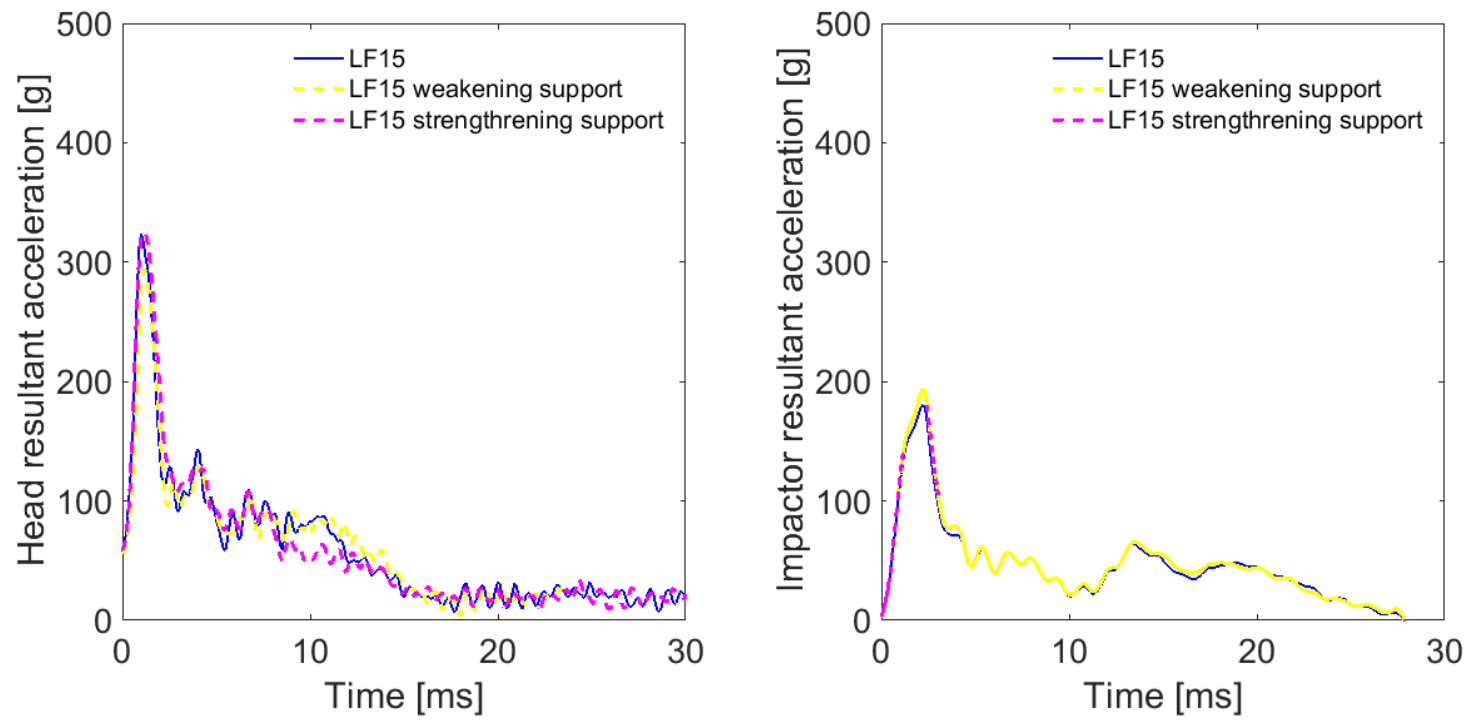

Figure 4.39 Time history of head acceleration when varying hood hinge support of LF15 (Left - full-scale impact; Right - impactor test) 

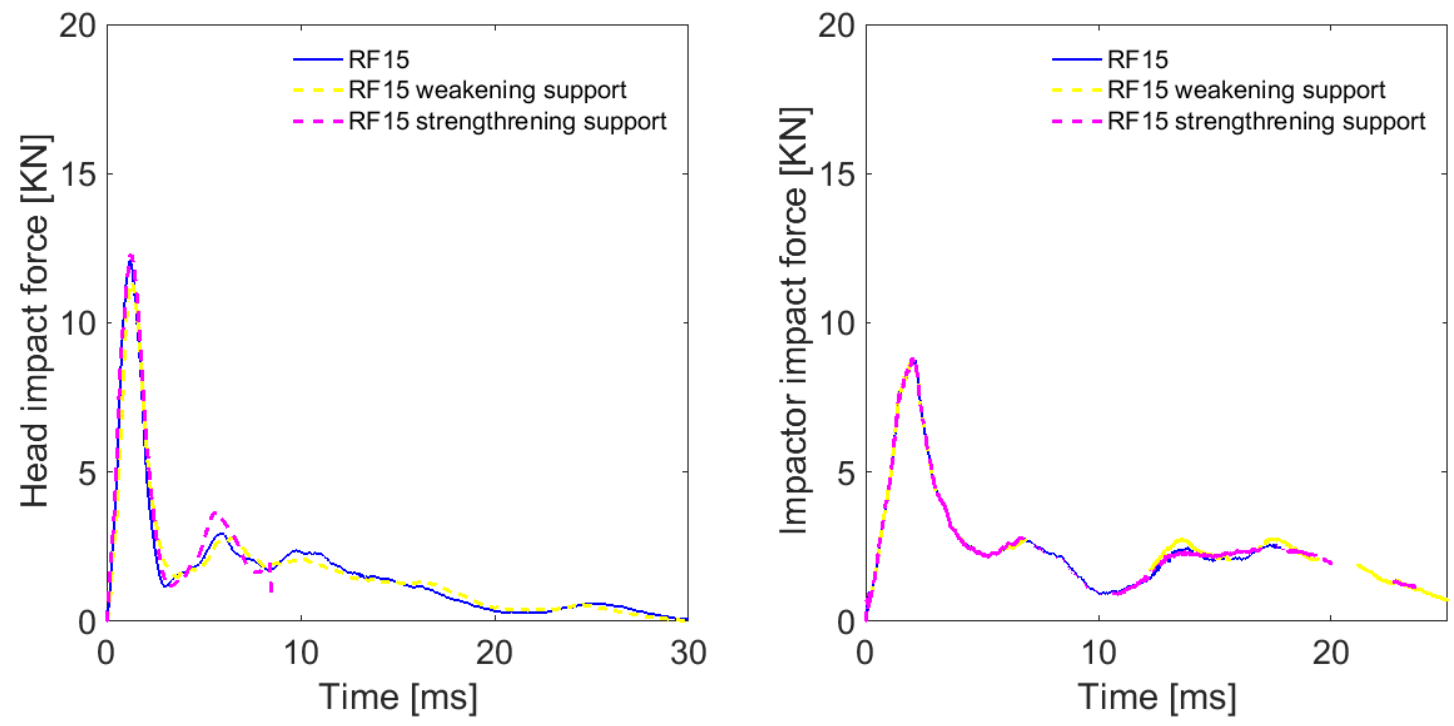

Figure 4.40 Time history of head impact force when varying hood hinge support of RF15 (Left - fullscale impact; Right - impactor test)
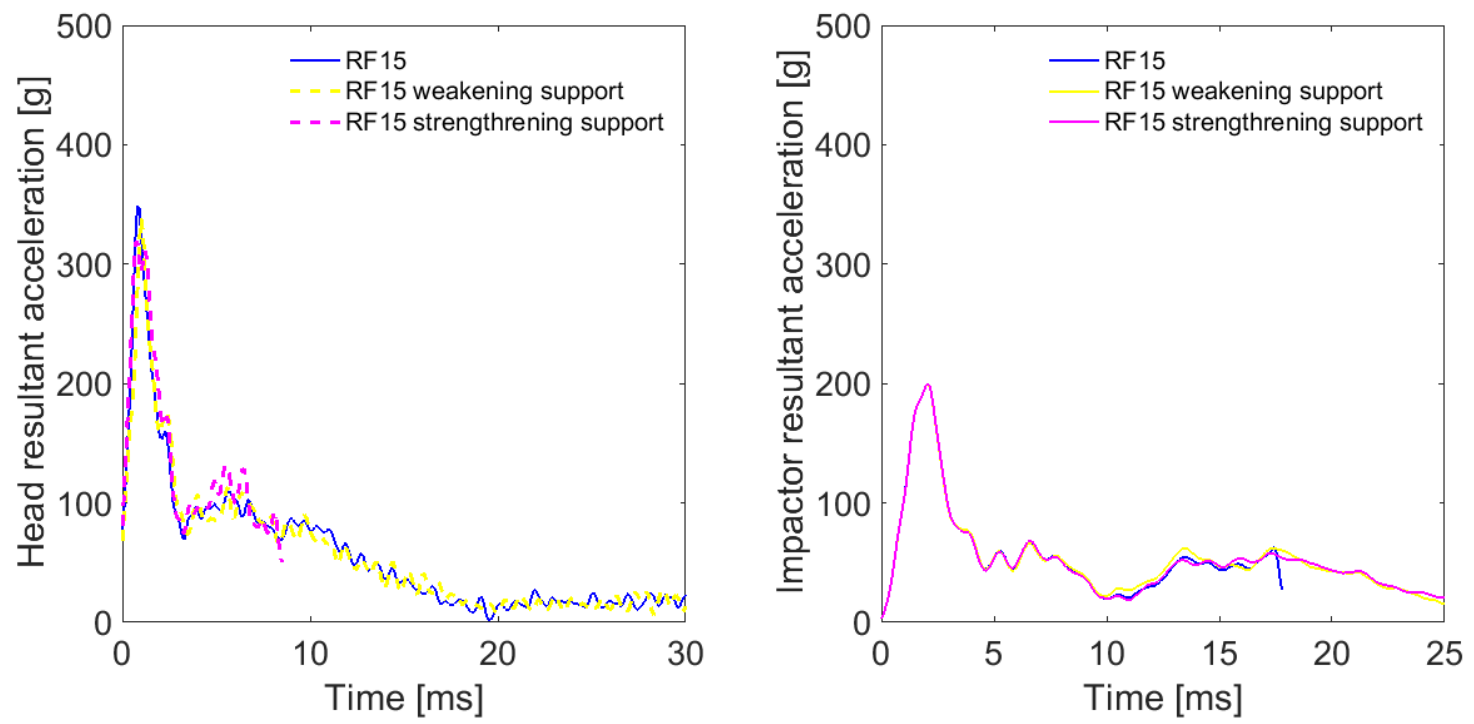

Figure 4.41 Time history of head acceleration when varying hood hinge support of RF15 (Left - full-scale impact; Right - impactor test) 


\section{Chapter 5 Effect of Factors on Pedestrian Head Response}

\subsection{Introduction}

Although the debate and critique of the limitations of the simplified sub-system test procedure have lasted years (Kerrigan et al. 2008; Hardy et al. 2007), it is still the only testing method used to evaluate vehicle aggressiveness because it is cost effective, repeatable, and easy to conduct. As a result, the industry is encouraged to optimize their vehicle design towards this component test procedure, instead of the full-scale pedestrian impact. However, previous PMHS tests (Kerrigan et al. 2008) observed higher HIC with lower head impact speeds than impactor tests, which has not been fully explained but suggested the potential problem. This leads to the question: how accurately can the sub-system test procedure represent the full-scale impact?

In chapter 4, impactor tests were not able to capture the head response of the full-scale impacts, and this observation was most obvious in the non-lateral impact scenarios. Also, when there was pre-deformation, vehicle design variations were found to have different impacts on head response and impactor response, and this observation was most obvious in the lateral impact scenarios.

Three main reasons may suggest why the impactor test did not capture the full-scale head response. First, compared to the full-scale, the sub-system test missed the head-neck interaction, the vehicle pre-deformation by the torso, and the head rotational kinematics. These factors could be classified as methodological differences between the impactor tests and the full-scale impacts. Second, the input of the testing conditions may differ. Specifically, the kinematics of the impactor tests may differ from the head kinematics before hitting the vehicle. Finally, there were 
differences in the model itself, including the geometry, material, and mass that could also contribute to these observations.

Recently, the FE human model and vehicle model provided high accuracy and enough complexity to answer this question that why impact tests cannot capture full-scale head response. One of the PFEMs, the THUMS pedestrian model, has been evaluated in many studies at the component level and the full-scale level. In chapter 3, this model was further evaluated on segmental velocity, acceleration, and whole-body injury, using geometrically personalized pedestrian models to consider the anthropometry dependence. The model was deemed biofidelic in the evaluation at both the component and the full-scale level. This model provided the appropriate tool to thoroughly study which factors play a role in the impactor test not capturing the full-scale head response.

The objective of this chapter is to further investigate the reasons why the impactor test does not capture the full-scale head response. The first main reason to explore is the kinematical differences between the impactors and the full-scale impacts. These differences will be matched to study the reasons for the differences, and then the major factors that could cause these observations will be investigated.

\subsection{Method and Material}

Three steps were performed to study the reasons for impactor tests not capturing fullscale head response. At the first step, the kinematics of impactors, including the head impact speed and impact angle, will be matched to the full-scale impacts to study the reason.

If matching the kinematics, the impactor tests still cannot capture the full-scale head response, then in the second step, the effect of all the potential major factors could cause this 
observation will be investigated. Six additional experiments were performed to study the effect of all the potential major factors that could cause the observation were investigated. Six additional experiments were performed to study the effect of all the potential major factors on the observation, and to determine which factors contributed most to the error between full-scale impact and the impactor test (Figure 5.1 and Table 5.1).

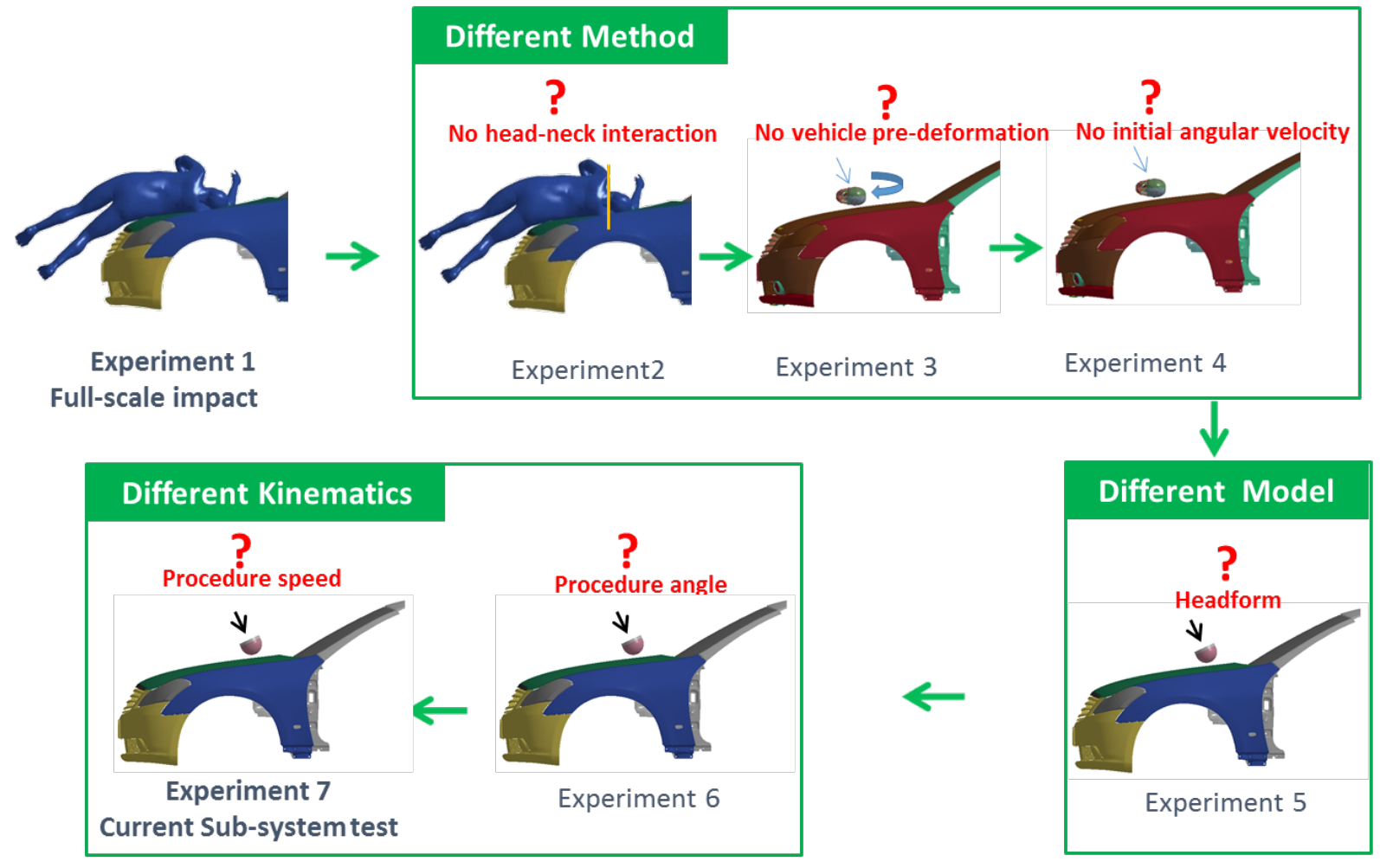

Figure 5.1 Experiments to study the effect of factors 
Table 5.1 Experiments to study the effect of factors

\begin{tabular}{|c|c|c|c|c|c|c|c|}
\hline Experiment & 1 & 2 & 3 & 4 & 5 & 6 & 7 \\
\hline Neck Interaction & $\mathrm{Y}$ & $\mathrm{N}$ & $\mathrm{N}$ & $\mathrm{N}$ & $\mathrm{N}$ & $\mathrm{N}$ & $\mathrm{N}$ \\
\hline Pre-deformation & $\mathrm{Y}$ & $\mathrm{Y}$ & $\mathrm{N}$ & $\mathrm{N}$ & $\mathrm{N}$ & $\mathrm{N}$ & $\mathrm{N}$ \\
\hline Angular velocity & $\mathrm{Y}$ & $\mathrm{Y}$ & $\mathrm{Y}$ & $\mathrm{N}$ & $\mathrm{N}$ & $\mathrm{N}$ & $\mathrm{N}$ \\
\hline Head Model & THUMS & THUMS & THUMS & THUMS & Impactor & Impactor & Impactor \\
\hline Impact angle & Full-scale & Full-scale & Full-scale & Full-scale & Full-scale & Impactor & Impactor \\
\hline Impact speed & Full-scale & Full-scale & Full-scale & Full-scale & Full-scale & $\begin{array}{l}\text { Full- } \\
\text { scale }\end{array}$ & Impactor \\
\hline Tested factor & - & $\begin{array}{c}\text { Neck } \\
\text { interaction }\end{array}$ & $\begin{array}{c}\text { Pre- } \\
\text { deformation }\end{array}$ & $\begin{array}{l}\text { Angular } \\
\text { velocity }\end{array}$ & $\begin{array}{c}\text { Model biofidelity } \\
\text { (geometry, mass, etc) }\end{array}$ & $\begin{array}{l}\text { Impact } \\
\text { angle }\end{array}$ & $\begin{array}{c}\text { Impact } \\
\text { speed }\end{array}$ \\
\hline
\end{tabular}

The error and absolute error were calculated to indicate the significance of the effect of each factor. Error is calculated from the mean deviation between head response of those experiments and of the full-scale impact, while the absolute error is the mean absolute deviation.

$$
\begin{gathered}
\text { Error }=\frac{1}{n} \sum_{i=1}^{N} e_{i} \\
\text { Error }=\frac{1}{n} \sum_{i=1}^{N}\left|e_{i}\right| \\
e_{i}=\frac{{\text { HIC (Experiment })_{i}-\text { HIC }(\text { full-scale })_{i}}_{\text {HIC }(\text { full-scale })_{i}}}{}
\end{gathered}
$$

The HIC is used as the main indicator for head response because it is the only measurement for the current pedestrian test procedure, and the BrIC was found to be insensitive to the vehicle stiffness variations in chapter 4 .

The paired-t test was performed to examine the significance of the difference between head response and impactor response, and p $=0.05$ was set as the threshold. To estimate the level of equality, the ration between head response and impactor responses were calculated and indicated by the slope of the linear line in the graphs. 
The last step is to investigate whether the hood structural stiffness increase with the level of vehicle pre-deformation. Six locations on the hood surface were selected to cover the center and the corner. Two types of vehicle hood material designs were covered, including the steel hood (hood outer and hood inner) and the aluminum hood (hood outer and hood inner).

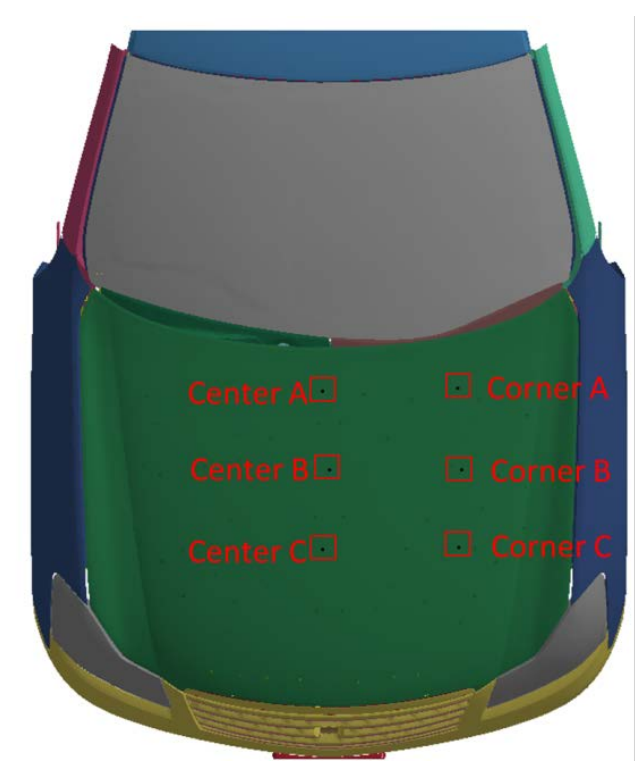

Figure 5.2 Selected impact locations

The motion of headforms was prescribed with constant speed $1 \mathrm{~m} / \mathrm{s}$ vertical to hood surface. The simulated maximum hood deflection levels are higher than $120 \mathrm{~mm}$ since the maximum hood deformation in chapter 4 could reach that level. 

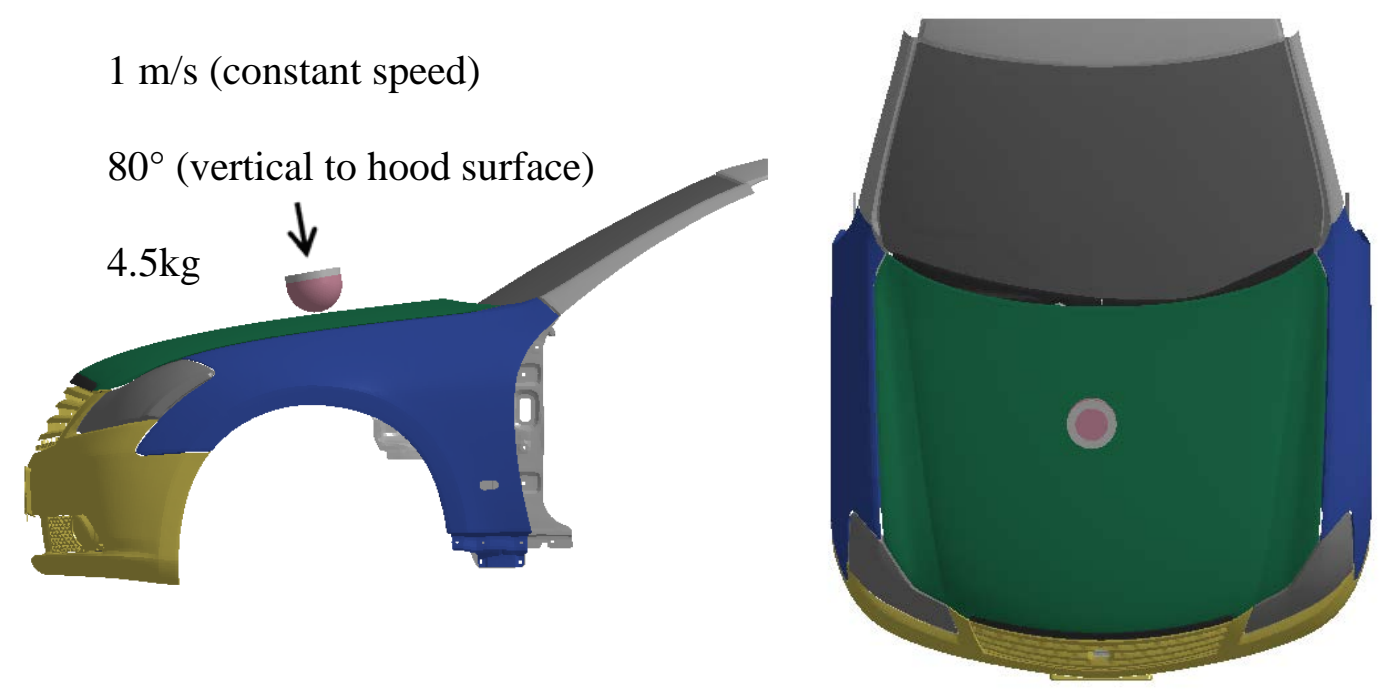

Figure 5.3 Impactor tests with prescribed constant speed

The polynomial regressions were performed to fit the force-deflection curves. $R^{2}$ indicates goodness of fit, and $\mathrm{p}_{1}$ and $\mathrm{p}_{2}$ indicate the significance of the coefficients. The structural stiffness is estimated as the slope $\mathrm{K}$ of the fitted curve and the stiffness of $10 \mathrm{~mm}$ and $100 \mathrm{~mm}$ deflection level were calculated.

$$
\begin{aligned}
& y=A x^{2}+B x \\
& k=2 A x+B
\end{aligned}
$$

\subsection{Results and Discussion}

\subsubsection{Matching Kinematics}

In impact configuration 1, which is the head impacting the windshield area without vehicle pre-deformation caused by torso before the head impact, after matching the input kinematics of impactors to the full-scale impact, the impactor tests captured the full-scale head response well (Figure 5.4). Before matching the kinematics, the p value was lower than 0.05, which suggested significant differences, but after matching kinematics, the $\mathrm{p}$ value increased to 
0.55, which suggested no significant difference. Also, the k value of the impactor tests increased from 0.61 to 0.99 , which suggested almost identical head response after matching the kinematic.
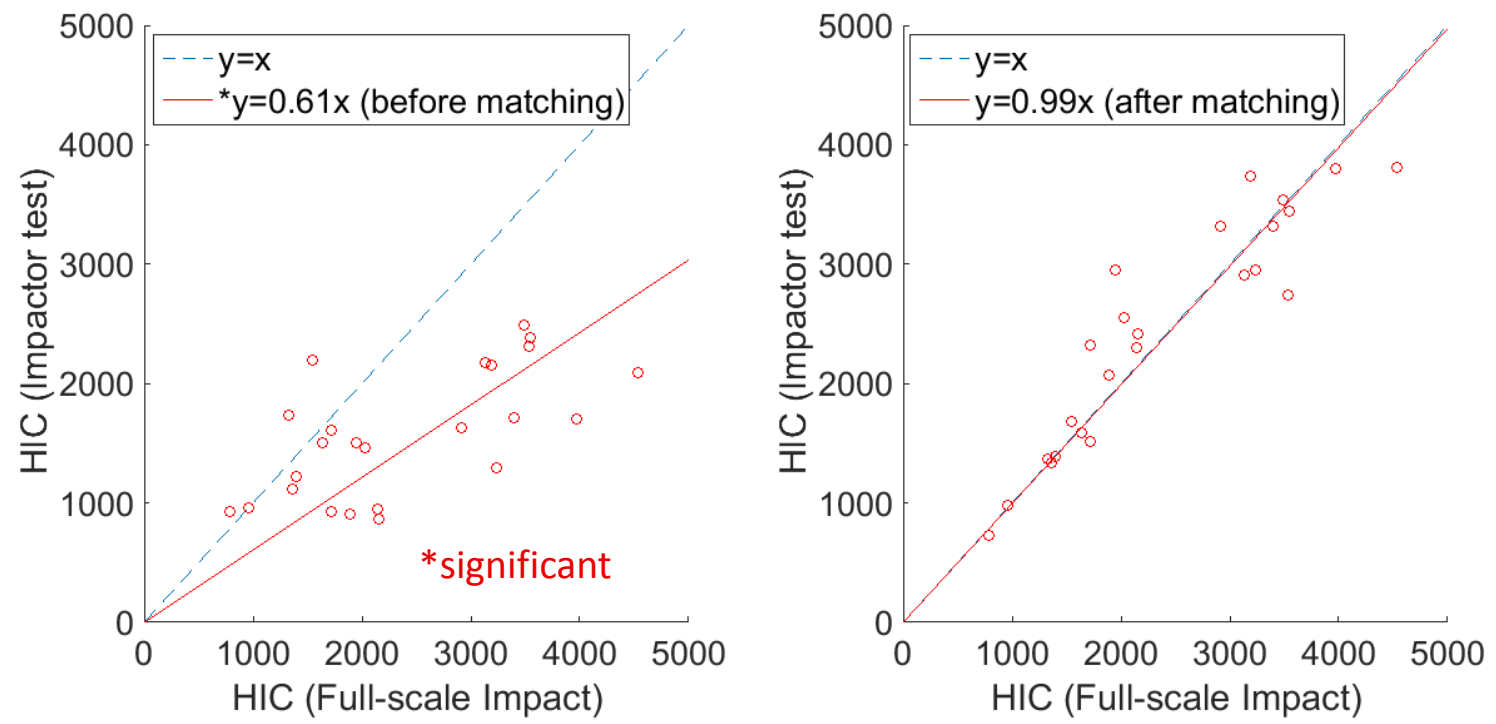

Figure 5.4 HIC of full-scale impacts and impactor tests (Left - before-matching kinematics; Right - after matching kinematics)

More changes were observed from the non-lateral impact scenarios than the lateral impact scenarios when matching the kinematics (Figure 5.5). In non-lateral impact scenarios, the original p value was lower than 0.05 , but increased to 0.23 , and the $\mathrm{k}$ value increased from 0.55 to 1.02; while in the lateral impact scenarios, the $\mathrm{p}$ value increased from 0.66 to 0.26 , but the $\mathrm{k}$ value only increased from 0.87 to 0.89 . 

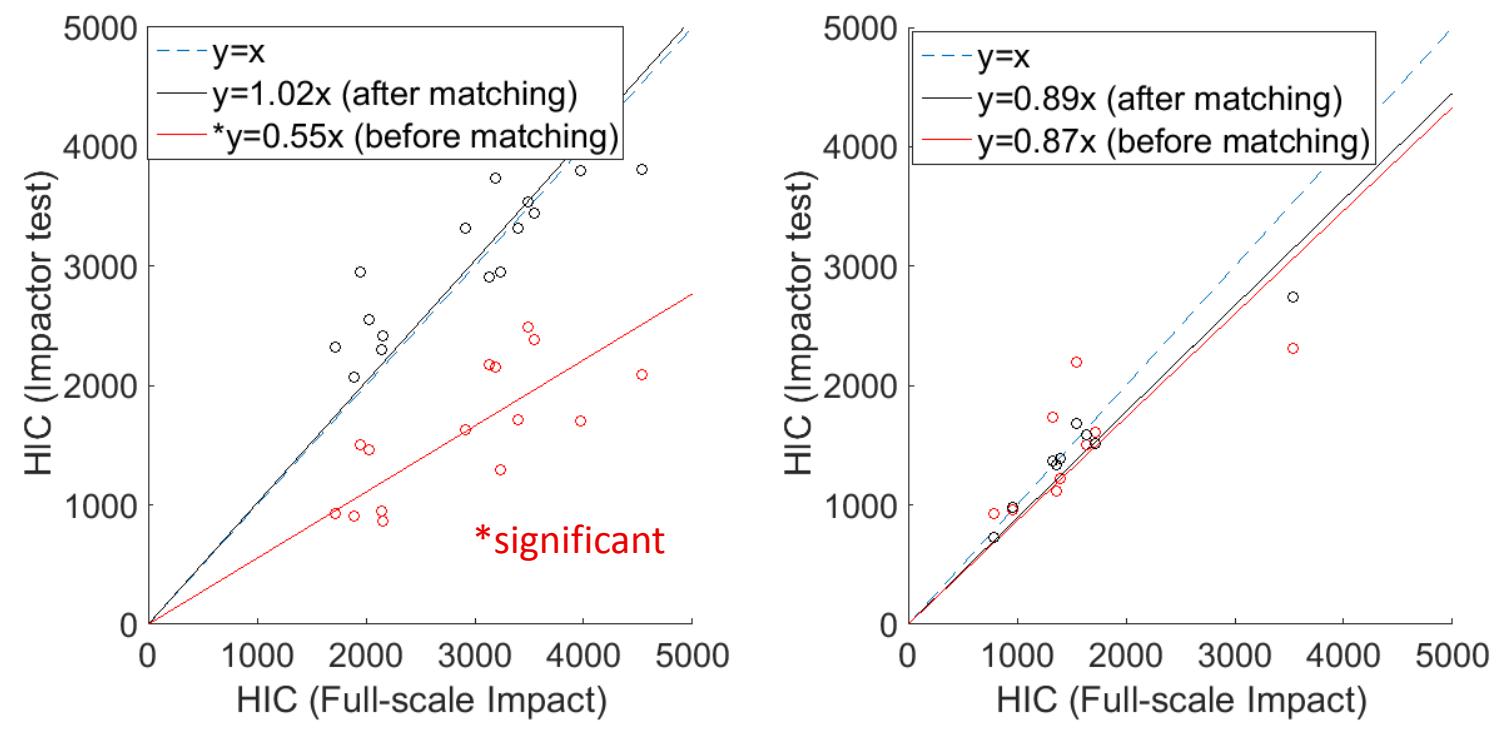

Figure 5.5 HIC of full-scale impacts and impactor tests (Left - Non-lateral impact scenarios; Right Lateral impact scenarios)

In impact configuration 2, which is the head impacting the pre-deformed hood area by the torso, after matching kinematics, the impactor tests still did not capture the HIC of the full-scale impact well (Figure 5.6). After matching kinematics, the p value increased from 0.01 to 0.03 , which still suggested significant difference. Also, the $\mathrm{k}$ value of the impactor tests increased from 0.38 to 0.74 , which is still far from 1 , though suggested an improvement.
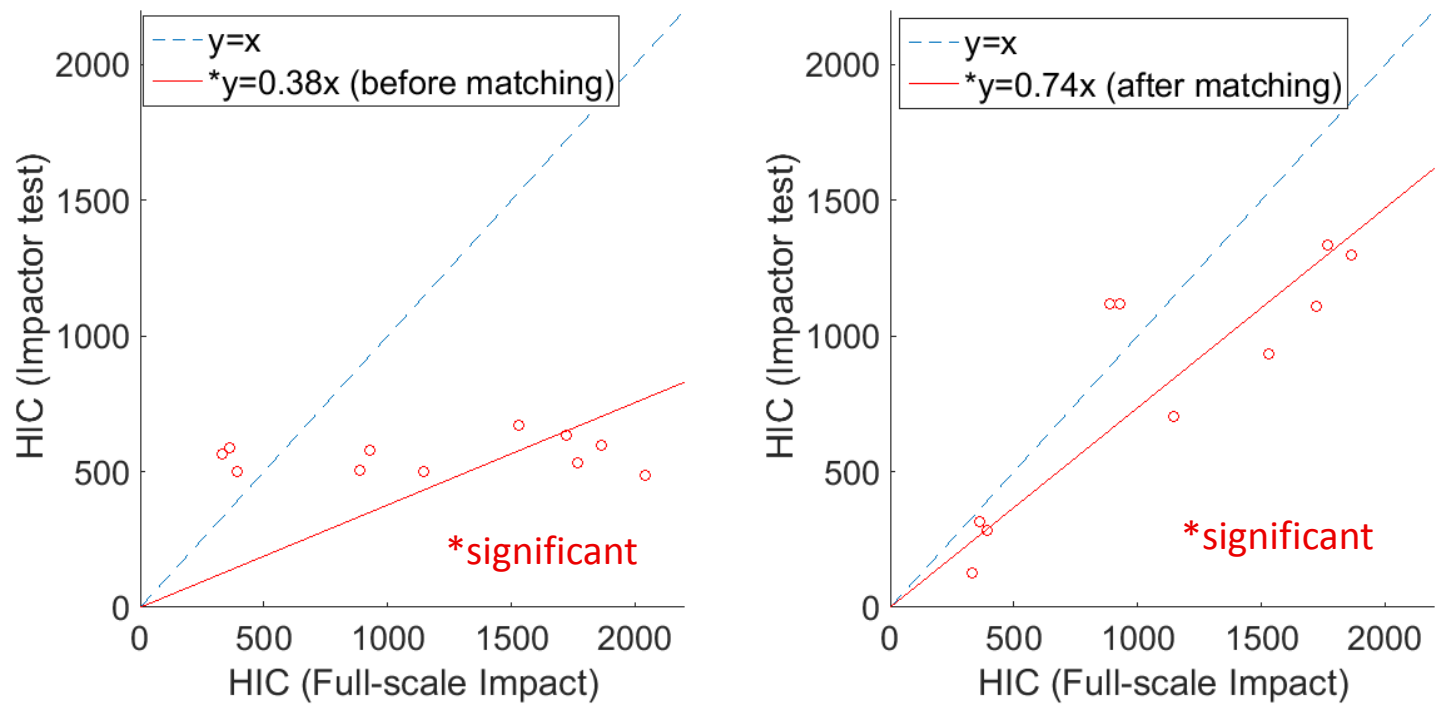
Figure 5.6 HIC of full-scale impacts and impactor tests (Left - before-matching kinematics; Right - after matching kinematics)

Also, less change was observed in the lateral impact scenarios when matching kinematics (Figure 5.7). In the non-lateral impact scenarios, after matching kinematics, the p value increased from 0.02 to 0.54 , which suggested no difference, and the k value increased from 0.33 to 0.83 , which suggested significant improvement. However, in the lateral impact scenarios, after matching kinematics, the p value decreased from 0.22 to 0.02 , which suggested a significant difference, and the $\mathrm{k}$ value only increased from 0.47 to 0.63 , which suggested the impactor tests still could not capture the HIC of the full-scale impact in the lateral impact scenarios. At the same time, more hood pre-deformation was observed in the lateral impact scenario than in the non-lateral impact (Figure 5.8). In baseline simulation RF15, the vertical maximum hood predeformation was $82.9 \mathrm{~mm}$, while in simulation RF15n90, the vertical maximum hood predeformation was only $9.9 \mathrm{~mm}$.
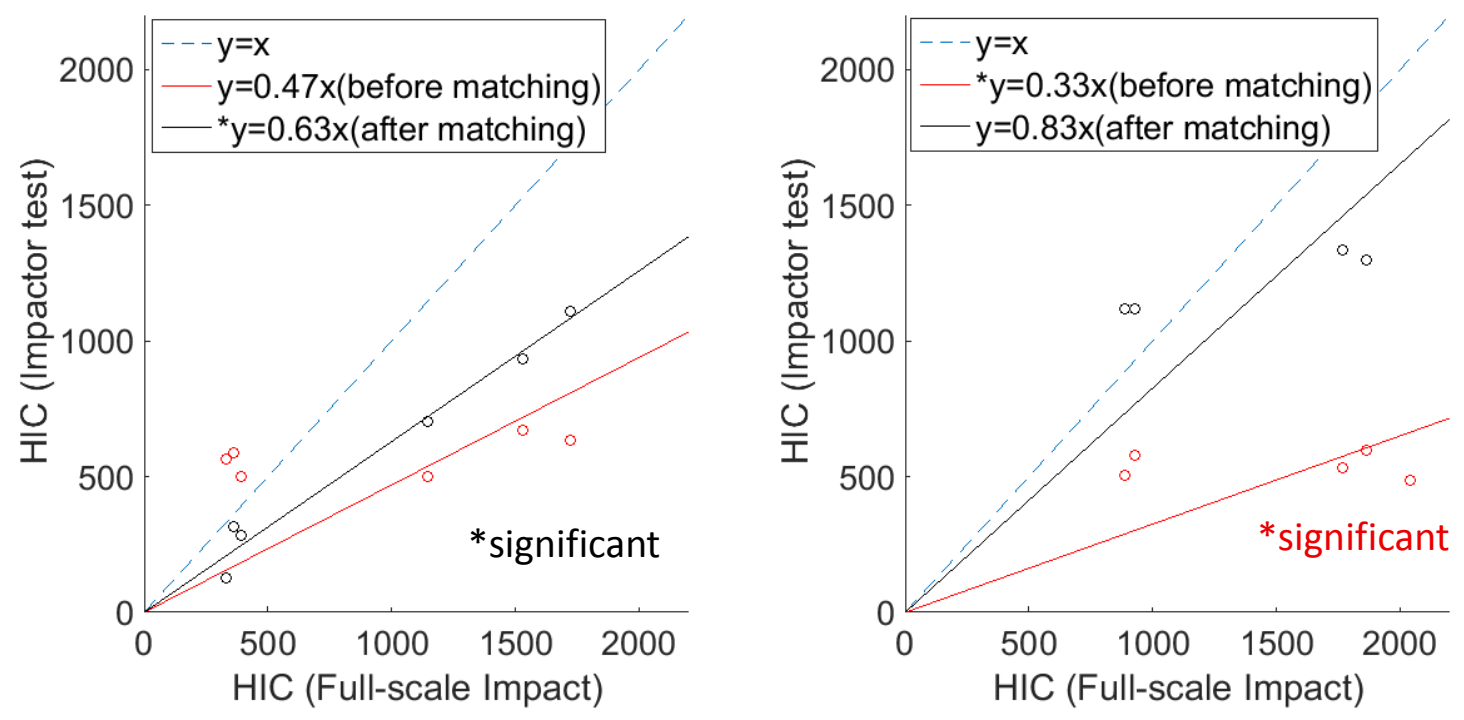

Figure 5.7 HIC of full-scale impacts and impactor tests (Left - Non-lateral impact scenarios; Right Lateral impact scenarios) 


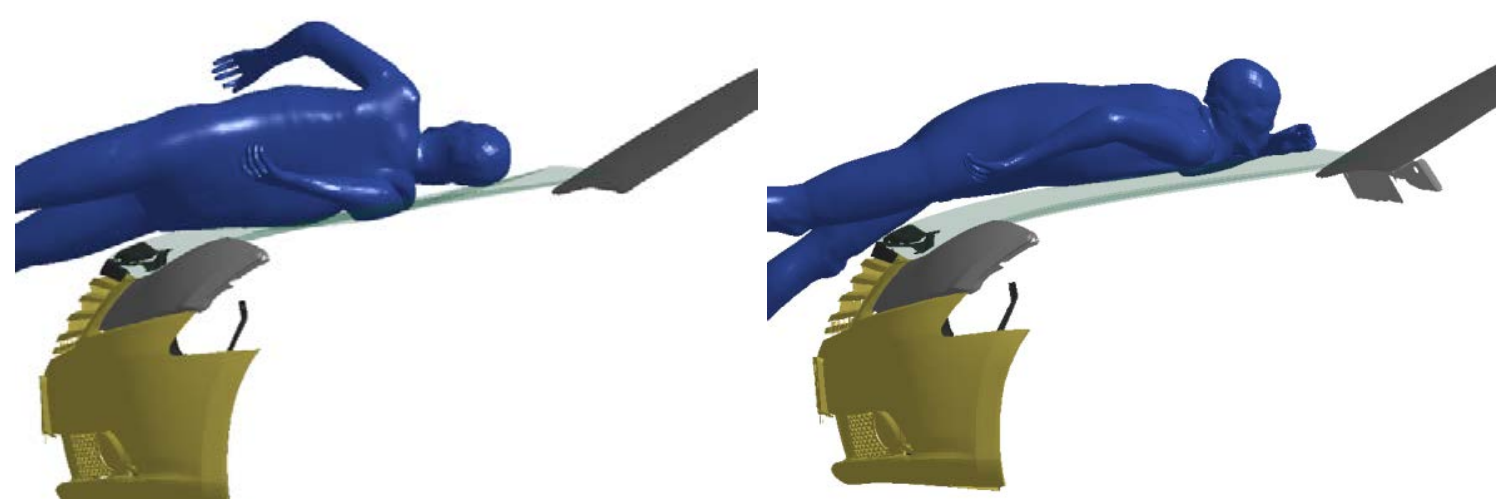

Figure 5.8 Vehicle pre-deformation in full-scale impact of impact configuration 2 (Left -Lateral impact scenario; Right -Non-lateral impact scenario)

\subsubsection{Effect of Factors on Head Response}

In impact configuration 2, after matching the kinematics, the impactor test could not capture the full-scale head response, which suggested that the difference of the head model or the difference of the testing method also contributed to the impactor tests not capturing the full-scale head response. The effects of all the potential factors are studied in the second step.

Table 5.2 listed the head or impactor response in all experiments for all 11 impact scenarios with impact configuration 2, including HIC and peak impact force. Table 5.3 and Table 5.4 listed the errors and absolute errors of each experiment of all 11 cases. Among all 11 cases, including 6 lateral impact scenarios and 5 non-lateral impact scenarios, the effects of each factor are not the same. The most significant change on the mean errors are from experiment 2 to experiment 3, and the mean error increased 18\%, which means the vehicle pre-deformations were found to significantly increase the HIC, and this was observed in 8 of 11 impact scenarios. However, the different errors in each case indicated the effect of factors depends on the impact scenarios. 
Table 5.2 Head or impactor response in all experiments

\begin{tabular}{|c|c|c|c|c|c|c|c|c|c|c|c|c|c|c|c|c|c|c|}
\hline \multirow{2}{*}{ No. } & \multirow{2}{*}{ Posture } & \multirow{2}{*}{ Anthropometry } & \multirow{2}{*}{ Direction } & \multirow{2}{*}{ Location } & \multicolumn{2}{|c|}{ Experiment 1} & \multicolumn{2}{|c|}{ Experiment 2} & \multicolumn{2}{|c|}{ Experiment 3} & \multicolumn{2}{|c|}{ Experiment 4} & \multicolumn{2}{|c|}{ Experiment 5} & \multicolumn{2}{|c|}{ Experiment 6} & \multicolumn{2}{|c|}{$\begin{array}{c}\text { Experiment } \\
7\end{array}$} \\
\hline & & & & & HIC & $\begin{array}{l}\text { Peak } \\
\text { force }\end{array}$ & HIC & $\begin{array}{l}\text { Peak } \\
\text { force }\end{array}$ & HIC & $\begin{array}{l}\text { Peak } \\
\text { force }\end{array}$ & HIC & $\begin{array}{l}\text { Peak } \\
\text { force }\end{array}$ & HIC & $\begin{array}{l}\text { Peak } \\
\text { force }\end{array}$ & HIC & $\begin{array}{l}\text { Peak } \\
\text { force }\end{array}$ & HIC & $\begin{array}{l}\text { Peak } \\
\text { force }\end{array}$ \\
\hline 1 & $\mathrm{LF}$ & 15 & 0 & 1 & 1529 & 8.8 & 1886 & 8.6 & 1081 & 8.2 & 1036 & 8.1 & 933 & 9.2 & 933 & 9.2 & 672 & 8.1 \\
\hline 2 & RF & 15 & 0 & 1 & 1720 & 10.1 & 1878 & 10.6 & 1125 & 9 & 1092 & 8.8 & 1111 & 10.1 & 1084 & 10.1 & 635 & 8.1 \\
\hline 3 & S & 15 & 0 & 2 & 394 & 2.7 & 351 & 2.7 & 289 & 2.7 & 288 & 2.8 & 285 & 3.9 & 293 & 3.9 & 501 & 5.3 \\
\hline 4 & S & 50 & 0 & 1 & 333 & 1.9 & 205 & 2.5 & 97 & 2.8 & 104 & 2.6 & 126 & 3.1 & 128 & 2.8 & 566 & 6.8 \\
\hline 5 & RF & 15 & 0 & 2 & 1148 & 4.7 & 1202 & 4.8 & 702 & 5.2 & 789 & 5 & 702 & 5.8 & 593 & 6.2 & 501 & 5.3 \\
\hline 6 & S & 15 & 0 & 1 & 361 & 3.5 & 348 & 3.8 & 362 & 4.1 & 353 & 4 & 316 & 4.3 & 284 & 4.7 & 586 & 5.9 \\
\hline 7 & S & 15 & 90 & 1 & 2041 & 7.2 & 1966 & 7 & 1802 & 9.2 & 2374 & 10 & 2114 & 10.6 & - & - & 486 & 5.8 \\
\hline 8 & $\mathrm{LF}$ & 15 & 90 & 1 & 1865 & 7.6 & 1734 & 7.4 & 1439 & 8 & 1778 & 8.5 & 1298 & 10.9 & 1392 & 9.8 & 598 & 6.7 \\
\hline 9 & $\mathrm{LF}$ & 15 & n90 & 1 & 926 & 4.9 & 742 & 4.7 & 818 & 5.3 & 844 & 5.3 & 1119 & 9.4 & 1104 & 8.1 & 581 & 6.8 \\
\hline 10 & $\mathrm{RF}$ & 15 & 90 & 1 & 1767 & 7.6 & 1596 & 7.4 & 1468 & 8.3 & 1836 & 8.9 & 1337 & 10.9 & 1527 & 9 & 533 & 7 \\
\hline 11 & RF & 15 & n90 & 1 & 888 & 4.4 & 693 & 4.5 & 807 & 5.4 & 1022 & 5.5 & 1118 & 10.2 & 1077 & 9 & 506 & 7.3 \\
\hline
\end{tabular}

Table 5.3 Errors of each experiment of all 11 cases in impact configuration 2

\begin{tabular}{cccccccc}
\hline Impact scenario & Experiment & Experiment & Experiment & Experiment & Experiment & Experiment & Experiment \\
& 1 & 2 & 3 & 4 & 5 & 6 & 7 \\
\hline LF15 & 0 & 0.233 & -0.293 & -0.322 & -0.390 & -0.390 & -0.560 \\
RF15 & 0 & 0.092 & -0.346 & -0.365 & -0.354 & -0.370 & -0.631 \\
S15corner & 0 & -0.109 & -0.266 & -0.269 & -0.277 & -0.256 & 0.272 \\
S50 & 0 & -0.384 & -0.709 & -0.688 & -0.622 & -0.616 & 0.700 \\
RF15corner & 0 & 0.047 & -0.389 & -0.313 & -0.389 & -0.483 & -0.564 \\
S15 & 0 & -0.036 & 0.003 & -0.022 & -0.125 & -0.213 & 0.623 \\
S1590 & 0 & -0.037 & -0.117 & 0.163 & 0.036 & - & -0.762 \\
LF1590 & 0 & -0.070 & -0.228 & -0.047 & -0.304 & -0.254 & -0.679 \\
LF15n90 & 0 & -0.199 & -0.117 & -0.089 & 0.208 & 0.192 & -0.373 \\
RF1590 & 0 & -0.097 & -0.169 & 0.039 & -0.243 & -0.136 & -0.698 \\
RF15n90 & 0 & -0.220 & -0.091 & 0.151 & 0.259 & 0.213 & -0.430 \\
Mean (all) & 0 & -0.071 & -0.247 & -0.160 & -0.200 & -0.231 & -0.282 \\
SD (all) & 0 & 0.166 & 0.193 & 0.257 & 0.273 & 0.267 & 0.544 \\
Mean (lateral) & 0 & -0.026 & -0.333 & -0.330 & -0.359 & -0.388 & -0.027 \\
SD (lateral) & 0 & 0.211 & 0.229 & 0.214 & 0.163 & 0.148 & 0.629 \\
Mean (non- & 0 & -0.124 & -0.145 & 0.044 & -0.009 & 0.004 & -0.588 \\
lateral) & & & & & & & \\
SD (non- & 0 & 0.081 & 0.055 & 0.113 & 0.256 & 0.234 & 0.175 \\
lateral) & 0 & & & & & & \\
\hline
\end{tabular}


Table 5.4 Absolute errors of each experiment of all 11 cases in impact configuration 2

\begin{tabular}{cccccccc}
\hline Impact scenario & Experiment & Experiment & Experiment & Experiment & Experiment & Experiment & Experiment \\
& 1 & 2 & 3 & 4 & 5 & 6 & 7 \\
\hline LF15 & 0 & 0.233 & 0.293 & 0.322 & 0.390 & 0.390 & 0.560 \\
RF15 & 0 & 0.092 & 0.346 & 0.365 & 0.354 & 0.370 & 0.631 \\
S15corner & 0 & 0.109 & 0.266 & 0.269 & 0.277 & 0.256 & 0.272 \\
S50 & 0 & 0.384 & 0.709 & 0.688 & 0.622 & 0.616 & 0.700 \\
RF15corner & 0 & 0.047 & 0.389 & 0.313 & 0.389 & 0.483 & 0.564 \\
S15 & 0 & 0.036 & 0.003 & 0.022 & 0.125 & 0.213 & 0.623 \\
S1590 & 0 & 0.037 & 0.117 & 0.163 & 0.036 & - & 0.762 \\
LF1590 & 0 & 0.070 & 0.228 & 0.047 & 0.304 & 0.254 & 0.679 \\
LF15n90 & 0 & 0.199 & 0.117 & 0.089 & 0.208 & 0.192 & 0.373 \\
RF1590 & 0 & 0.097 & 0.169 & 0.039 & 0.243 & 0.136 & 0.698 \\
RF15n90 & 0 & 0.220 & 0.091 & 0.151 & 0.259 & 0.213 & 0.430 \\
Mean (all) & 0 & 0.139 & 0.248 & 0.224 & 0.291 & 0.312 & 0.572 \\
SD (all) & 0 & 0.109 & 0.192 & 0.197 & 0.135 & 0.150 & 0.154 \\
Mean (lateral) & 0 & 0.150 & 0.334 & 0.330 & 0.359 & 0.388 & 0.558 \\
SD (lateral) & 0 & 0.135 & 0.228 & 0.214 & 0.163 & 0.148 & 0.149 \\
Mean (non- & 0 & 0.124 & 0.145 & 0.098 & 0.210 & 0.199 & 0.588 \\
lateral) & & & & & & & \\
SD (non- & 0 & 0.081 & 0.055 & 0.058 & 0.103 & 0.049 & 0.175 \\
lateral) & & & & & & & \\
\hline
\end{tabular}

In 6 lateral impact scenario cases, vehicle pre-deformation caused by the torso before head impact and head impact speed were the most notable factors. Vehicle pre-deformation caused a 31\% error change and a 16\% absolute error change (Figure 5.9 and Figure 5.10), while head impact speed caused 36\% error change and a 17\% absolute error change. The effect of initial angular velocity, the difference impactor model and head model, and the head impact angle were found to be low, with error changes of $0.3 \%, 2.9 \%$, and $2.9 \%$ respectively. 


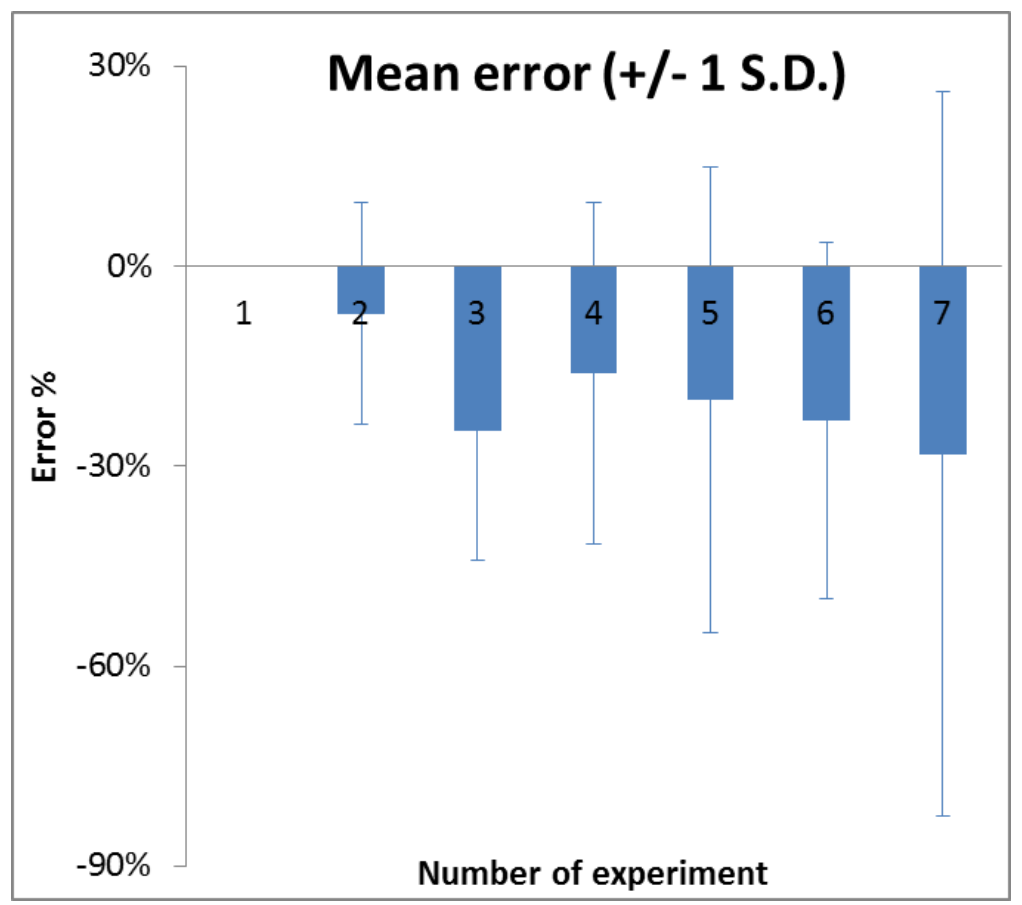

Figure 5.9 Errors of each experiment of all 11 cases in impact configuration 2

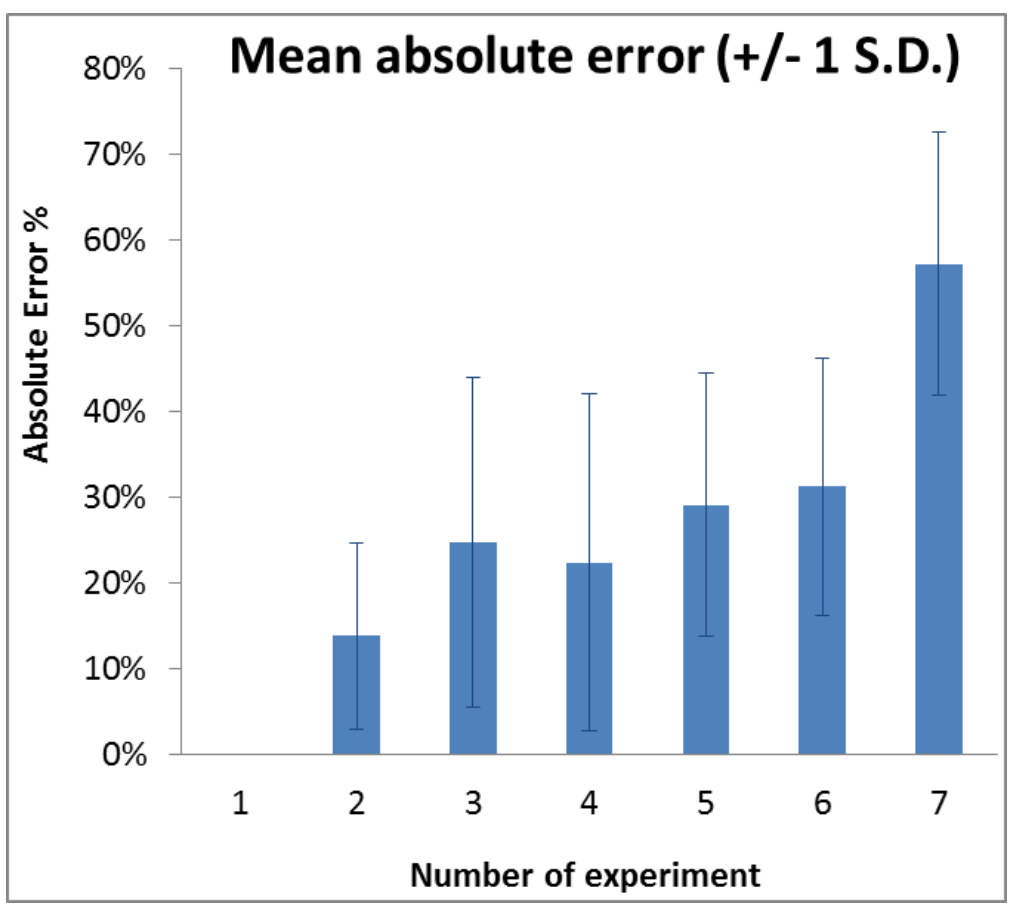

Figure 5.10 Absolute errors of each experiment of all 11 cases in impact configuration 2 
In 3 of the 6 lateral impact scenario cases, the head-neck interaction increased the HIC, which suggested the effect of the head-neck interaction is complex and the neck is not necessarily in tension in lateral impact scenarios (Figure 5.11 and 5.12).

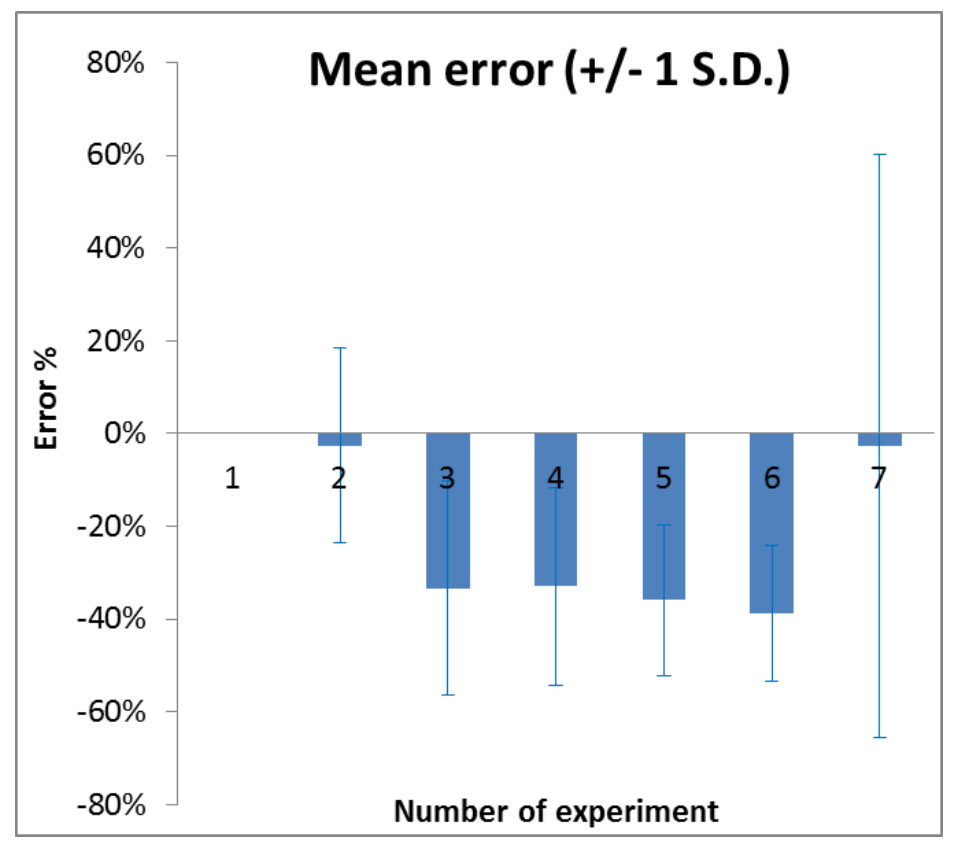

Figure 5.11 Errors of each experiment of 6 lateral impact cases in impact configuration 2

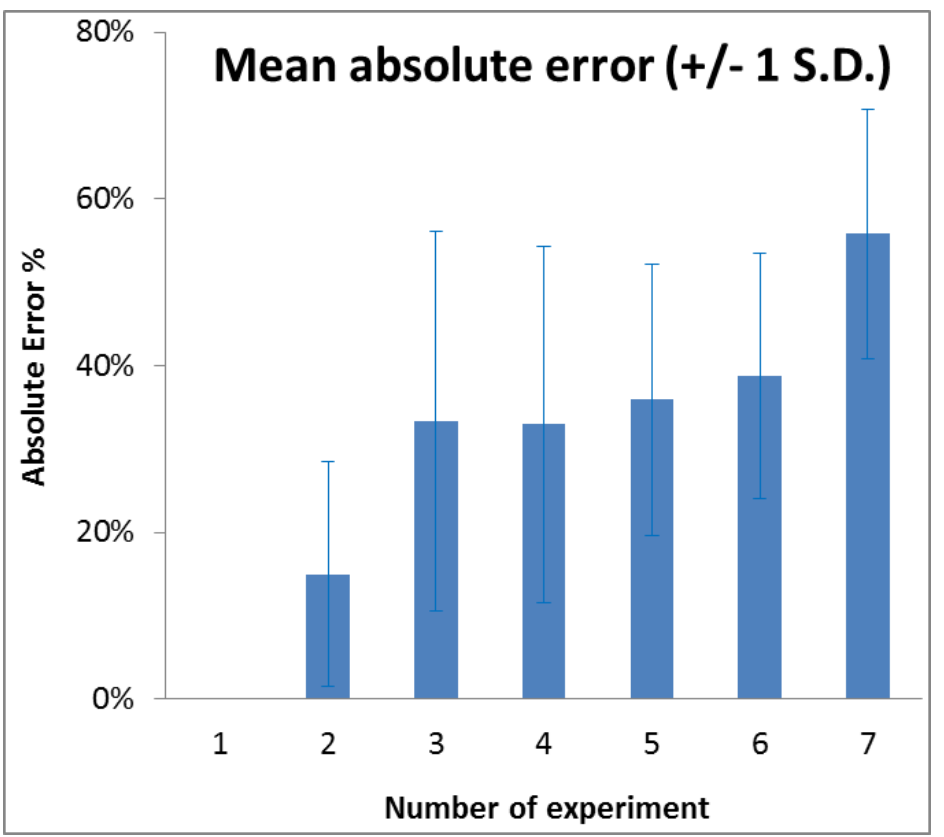


Figure 5.12 Absolute errors of each experiment of 6 lateral impact cases in impact configuration 2

The time history of experiment 2 and 3 in impact scenario LF15 (Figure 5.13) and RF15 (Figure 5.14) showed decreased peak force (8.2 KN than 8.6 KN for LF15; $9 \mathrm{KN}$ than $10.6 \mathrm{KN}$ for RF15) and HIC (1081 than 1886 for LF15; 1125 than 1878 for RF15) when removing the vehicle pre-deformation.
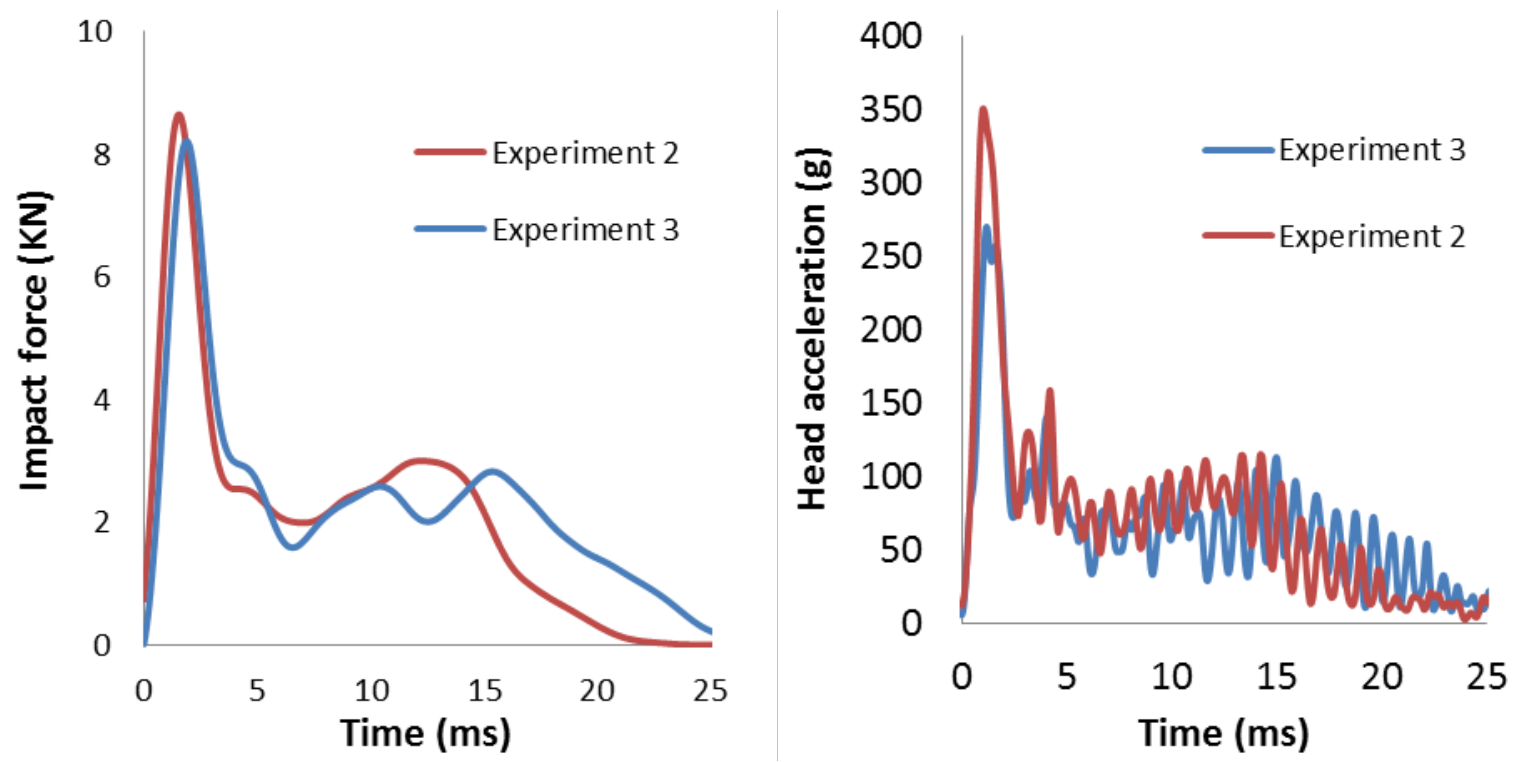

Figure 5.13 Time history of experiment 2 and 3 in impact scenario LF15 (Left - head impact force; Right - head acceleration) 

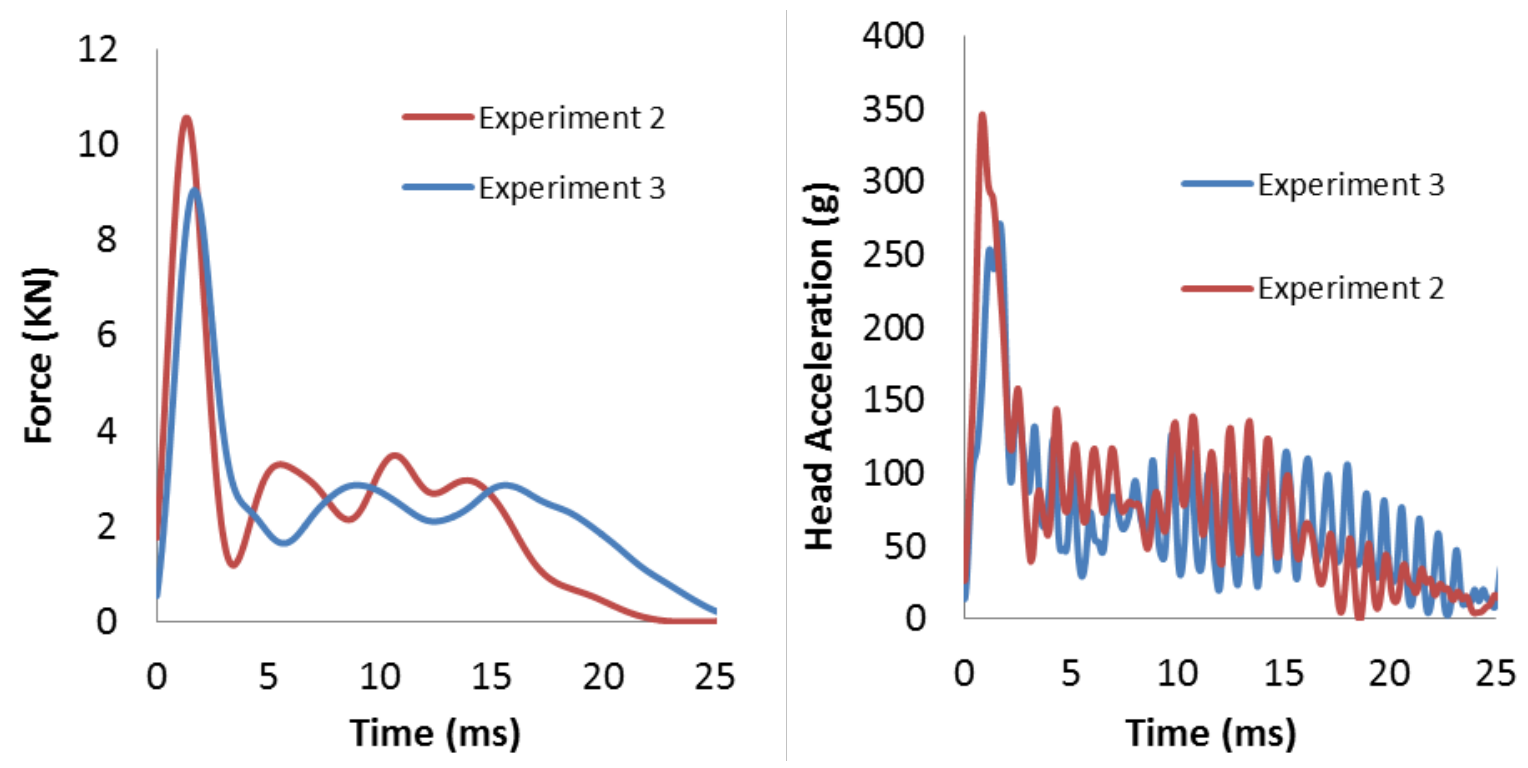

Figure 5.14 Time history of experiment 2 and 3 in impact scenario RF15 (Left - head impact force; Right - head acceleration)

In 5 non-lateral impact scenario cases, head impact speed was the most notable factor, which caused a 58.4\% error change and a 38.9\% absolute error change (Figures 5.15 and 5.16).

In all the 5 non-lateral impact scenario cases, the head-neck interaction increased the HIC, which suggested different head-neck interactions in lateral and non-lateral impact configurations. In the 5 non-lateral impact scenarios, the neck was found to be in compression during the fullscale vehicle-head impact.

Lower than in the lateral impact scenario, in non-lateral impact scenarios, the vehicle predeformation only caused a $2.1 \%$ error change and a $2.1 \%$ absolute error change. Figure 5.17 and Figure 5.18 showed the time history of experiment 2 and 3 in impact scenario LF1590 and RF1590, including force and acceleration. When removing the vehicle pre-deformation, higher peak forces (8 KN than 7.4 KN for LF1590; 8.3 KN than 7.4 KN for RF1590) and lower HIC (1439 than 1734 for LF1590; 1468 than 1596 for RF1590) were observed. 


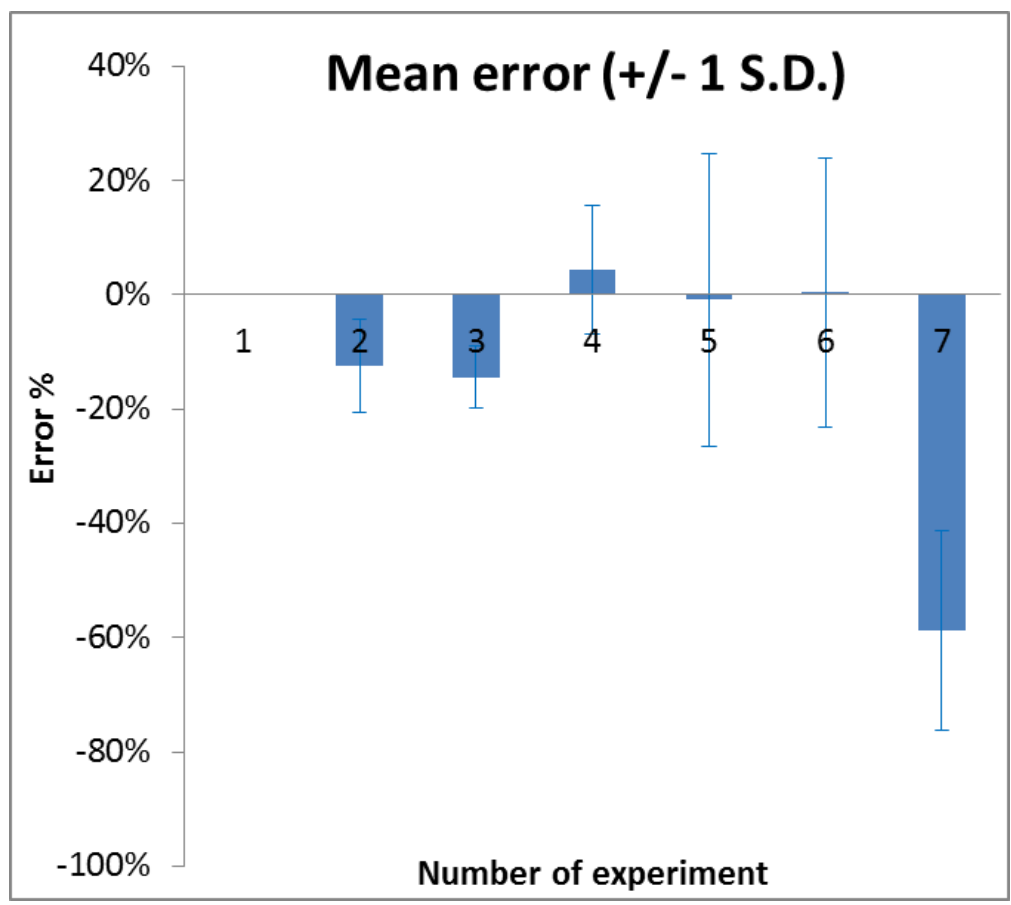

Figure 5.15 Errors of each experiment of 5 non-lateral impact cases in impact configuration 2

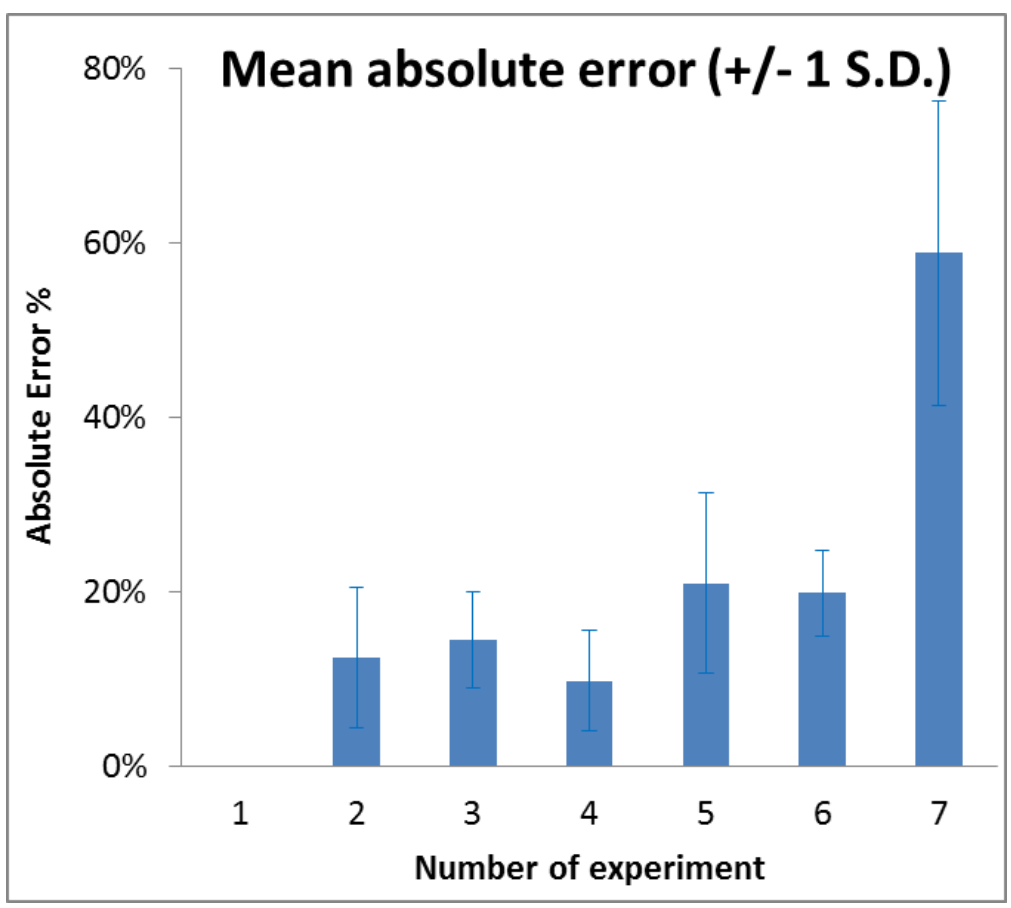

Figure 5.16 Absolute errors of each experiment of 5 non-lateral impact cases in impact configuration 2 

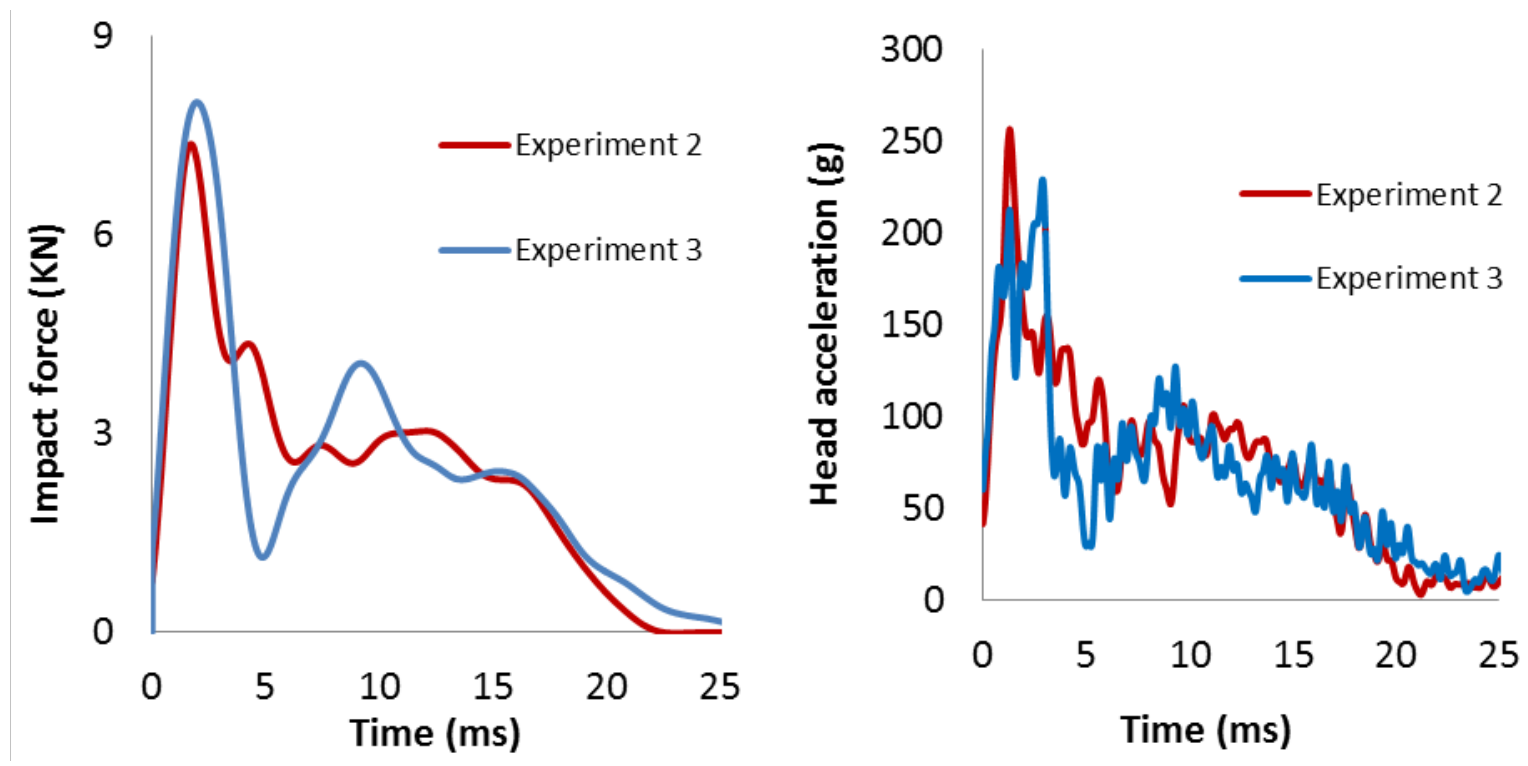

Figure 5.17 Time history of experiment 2 and 3 in impact scenario LF1590 (Left - head impact force; Right - head acceleration)
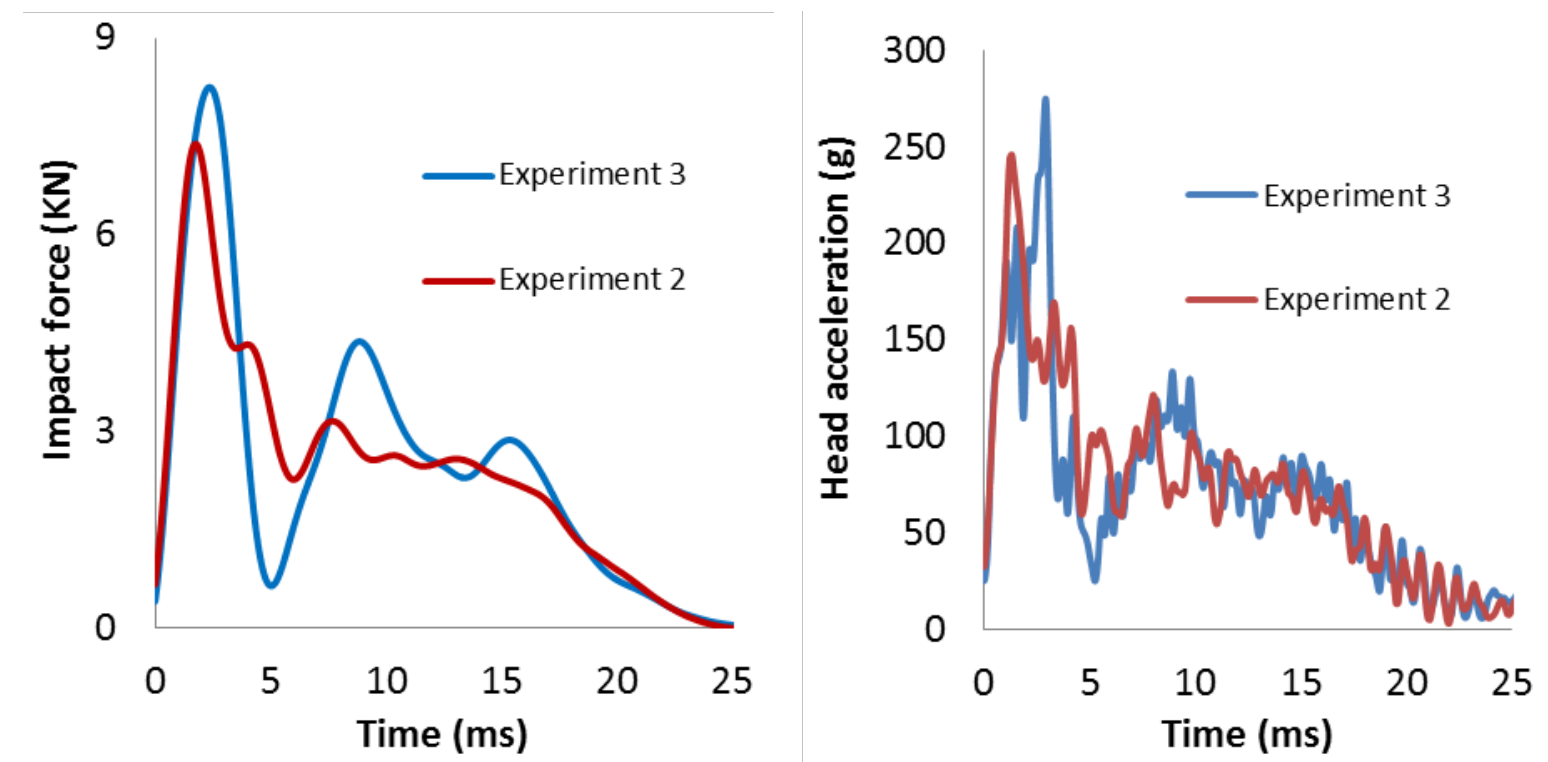

Figure 5.18 Time history of experiment 2 and 3 in impact scenario RF1590 (Left - head impact force; Right - head acceleration)

\subsubsection{Structural Stiffness of Hood Surface}

For both types of vehicle hood material designs, high $\mathrm{R}^{2}(>0.95)$ and low $\mathrm{p}$ values $\left(\mathrm{p}_{1}<0.05 ; \mathrm{p}_{2}<0.05\right)$ were obtained. Also, the $\mathrm{A}$ and $\mathrm{B}$ values are positive in the regressions. The 
estimated structural stiffness at $100 \mathrm{~mm}$ deflection level is 2.1 times and 3.6 times of stiffness at $10 \mathrm{~mm}$ deflection level, for steel hood and aluminum hood material design.

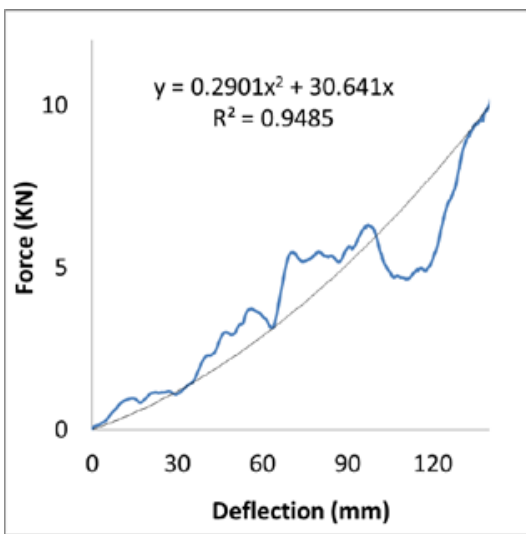

Center A $\left(p_{1}<0.05 ; p_{2}<0.05\right)$

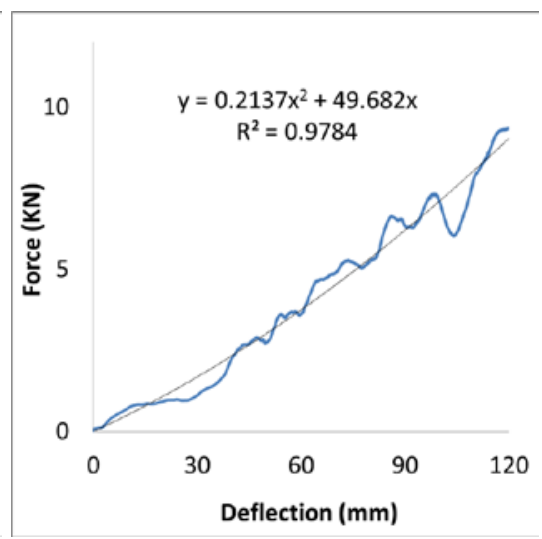

Center B $\left(p_{1}<0.05 ; p_{2}<0.05\right)$

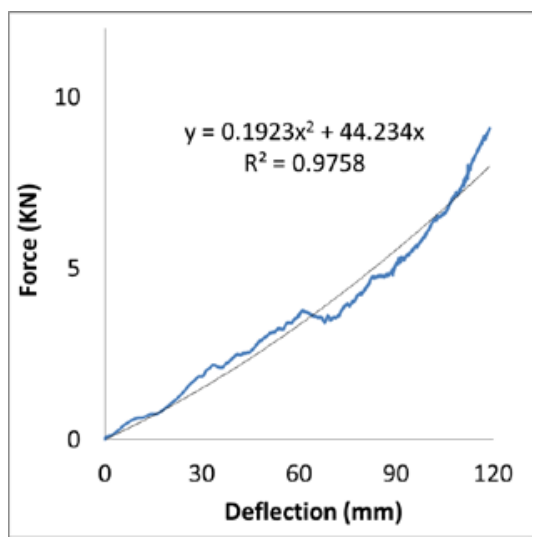

Center $C\left(p_{1}<0.05 ; p_{2}<0.05\right)$

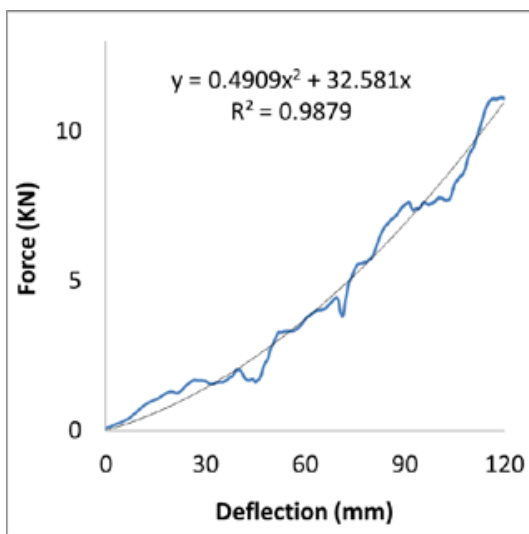

Corner A $\left(p_{1}<0.05 ; p_{2}<0.05\right)$

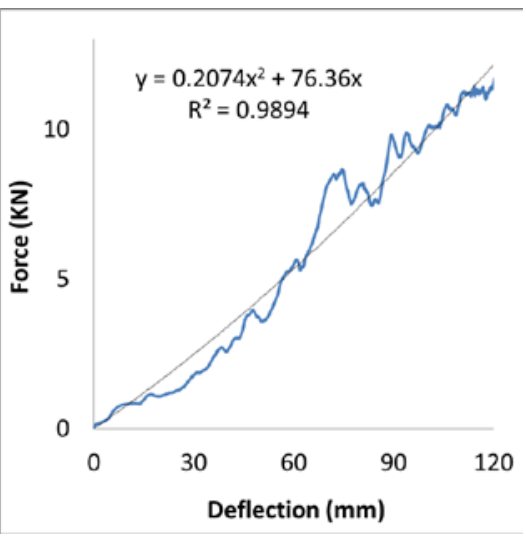

Corner $B\left(p_{1}<0.05 ; p_{2}<0.05\right)$

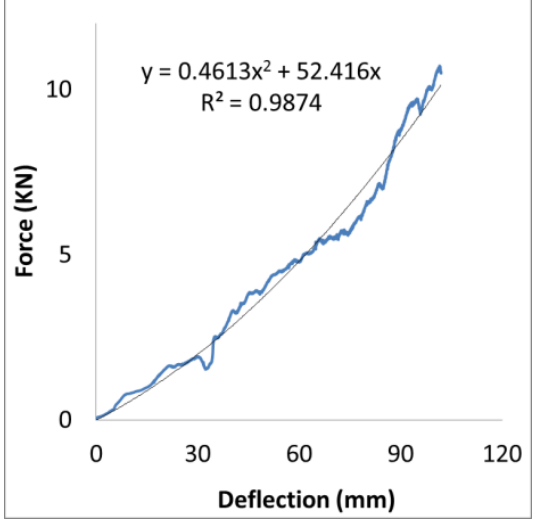

Corner $C\left(p_{1}<0.05 ; p_{2}<0.05\right)$

Figure 5.19 Force-deflection response of steel hood 

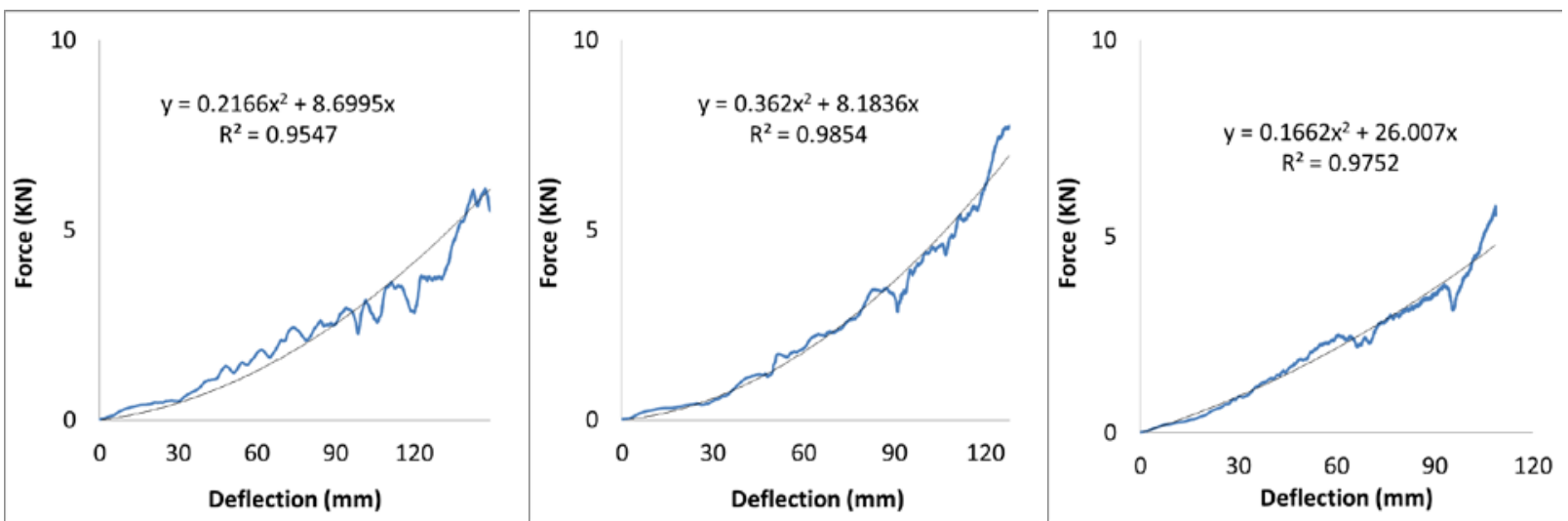

Center A $\left(p_{1}<0.05 ; p_{2}<0.05\right)$

Center B $\left(p_{1}<0.05 ; p_{2}<0.05\right)$

Center $C\left(p_{1}<0.05 ; p_{2}<0.05\right)$

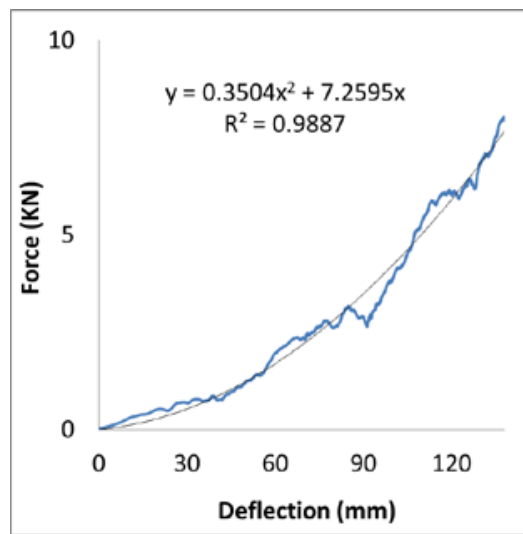

Corner A $\left(p_{1}<0.05 ; p_{2}<0.05\right)$

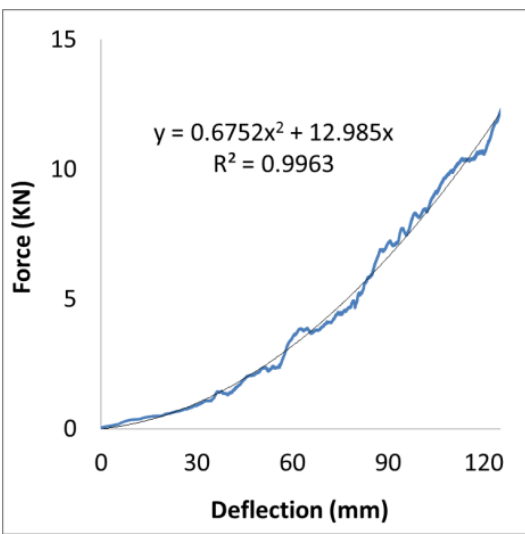

Corner B $\left(p_{1}<0.05 ; p_{2}<0.05\right)$

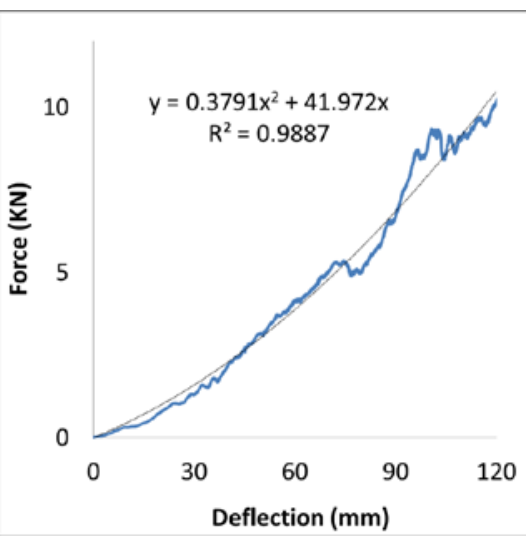

Corner $C\left(p_{1}<0.05 ; p_{2}<0.05\right)$

Figure 5.20 Force-deflection response of aluminum hood 
Table 5.5 Structural stiffness at $10 \mathrm{~mm}$ and $100 \mathrm{~mm}$ deflection level

\begin{tabular}{ccccc}
\hline & \multicolumn{4}{c}{ Structural stiffness (N/mm) } \\
\cline { 2 - 5 } Impact Location & \multicolumn{2}{c}{ Steel hood } & \multicolumn{2}{c}{ Aluminum hood } \\
& $\begin{array}{c}10 \mathrm{~mm} \\
\text { deformation }\end{array}$ & $\begin{array}{c}100 \mathrm{~mm} \\
\text { deformation }\end{array}$ & $\begin{array}{c}10 \mathrm{~mm} \\
\text { deformation }\end{array}$ & $\begin{array}{c}100 \mathrm{~mm} \\
\text { deformation }\end{array}$ \\
\hline Center A & 36.4 & 88.6 & 13 & 52 \\
Center B & 50 & 92.4 & 15.4 & 80.6 \\
Center C & 48.1 & 82.7 & 29.3 & 59.2 \\
Corner A & 42.4 & 130.8 & 14.3 & 77.3 \\
Corner B & 80.5 & 117.9 & 26.5 & 148 \\
Corner C & 61.6 & 144.7 & 49.6 & 117.8 \\
\hline Average & 53.2 & 109.5 & 24.7 & 89.2 \\
\hline
\end{tabular}

\subsection{Discussion}

\subsubsection{Matching Kinematics}

In impact configuration 1, after matching the kinematics, the impactor tests could capture the full-scale head response. This suggested that, in terms of methodology, the sub-system test procedure has good potential to predict pedestrian head response by inputting realistic pedestrian head impact conditions. More improvement on matching HIC of impact tests to full-scale impacts after matching kinematics was observed in non-lateral impact scenarios, mainly because before matching kinematics there was more difference on kinematics between head and impactors $(11.1 \mathrm{~m} / \mathrm{s})$ in non-lateral impacts $(16.1 \pm 0.7)$ than in lateral impacts $(10.1 \pm 3.1)$, as shown in Table 4.4 of chapter 4.

However, in impact configuration 2, after matching the kinematics, the impactor tests still did not capture the head response. This suggested that ignoring the vehicle pre-deformation by the torso before the head impact was a methodological limitation of the sub-system test 
procedure. In lateral impact scenarios, after matching kinematics, there were more improvements on matching the HIC of the impact tests to the full-scale impacts, mainly because there were more pre-deformation in the lateral impact, as shown in Figure 5.8.

\subsubsection{Effect of Factors on Head Response}

The effect of the neck was found to depend on the impact scenario. In lateral impact scenarios, generally the shoulder impacted the vehicle first, which resulted in the neck being in tension when the head impacted the vehicle, and thus in experiment 2, when the head-neck interaction was removed, the HIC increased. While in non-lateral impact scenarios, the thorax or back impacted the hood almost at the same time as the head impacted. After which, the head was resisted by the hood but the upper body continued to move forward and downward towards the vehicle due to inertia, which resulted in the neck being in compression. Thus, when the headneck interaction was removed, the HIC decreased.

The effect of vehicle pre-deformation caused by the torso was also found to depend on the impact scenarios. In lateral impact scenarios, the vehicle pre-deformation increased the HIC by $31 \%$, while in non-lateral impact scenarios, the vehicle pre-deformation only increased HIC by $2.1 \%$. In lateral impacts, there were more vehicle pre-deformations (Figure 5.8) which already absorbed the impact energy, and thus at the time of head impact, the hood could be structurally stiffer and was closer to rebounding. While in non-lateral impacts, there was less vehicle predeformation caused by the torso and less energy absorbed before the head impact, which might have resulted in a less stiff hood and thus less increase on HIC than in lateral impacts.

The more vehicles hood deform, the higher probability the bottoming out phenomenon could happen, which means the head impact might reach the underneath stiff component like 
engine. Therefore, the impactor tests causing less vehicle deformation than full-scale impacts could be a potential limitation of current regulation because those vehicle designs that could cause bottoming out in full-scale impact will probably not be detected in component test.

Kinematics was always one of the most significant factors, in both lateral and non-lateral impacts, and the effect was found to be mainly from the head impact speed, instead of impact angle. In non-lateral impacts, the head impact speeds were $16.1 \pm 0.7 \mathrm{~m} / \mathrm{s}$, which was $45 \%$ higher than the impactor test speed of $11.1 \mathrm{~m} / \mathrm{s}$. In lateral impacts, high variability on head impact speed was observed due to elbow impacts which happened in several impact scenarios. The impact energy changed with the square of impact speed, and under the assumption of a linear spring, the HIC values from two impactor tests performed on a structure of constant stiffness was related via the following equation 5.4 derived by Searson et al. (2009). In this equation, the value $H I C_{1}$ corresponded to an impact speed of $v_{1}$ (normal component of the impactor velocity to the vehicle surface) and headform mass $m_{1}$, and similarly for $H I C_{2}$. In another study (Mizuno and Kajzer 2000), the relation between the HIC and dynamic deformation $x_{d}$ was obtained as equation 5.5 , where $v_{0}$ is the initial velocity $(\mathrm{m} / \mathrm{s})$. In both studies, the theoretical HIC calculated increased markedly with impact velocity.

$$
\begin{gathered}
\left(\frac{H I C_{1}}{H I C_{2}}\right)=\left(\frac{m_{1}}{m_{2}}\right)^{-0.75}\left(\frac{v_{1}}{v_{2}}\right)^{2.5} \\
H I C=0.001882 V_{0}^{4} X_{d}^{-1.5}
\end{gathered}
$$

The head impact angle was not found to be a significant factor. In this study, in all 11 cases in impact configuration 2 , the head impact angles were $62.9^{\circ} \pm 10.4^{\circ}$, which was very close to the impact angle of impactor tests $65^{\circ}$. This means within one standard deviation of the impact angle, the normal component of impact speed (equation 5.6, 9.7 ${ }^{\circ}$ is the hood inclination 
for the mid-size sedan) was within 0.89 to 0.99 times of the head resultant speed, which is very close to the normal component of 0.96 times of impact speed.

$$
V_{\text {normal }}=V^{*} \sin \left(\alpha+9.7^{\circ}\right)
$$

\subsubsection{Structural Stiffness of Hood Surface}

For both types of vehicle hood material designs, high $\mathrm{R}^{2}(>0.95)$ and low $\mathrm{p}$ values $\left(\mathrm{p}_{1}<0.05 ; \mathrm{p}_{2}<0.05\right)$ indicated good fitting and significant non-linear relationship between force and deflection. The positive A values indicated the structural stiffness increases with more deformation. The estimated structural stiffness at $100 \mathrm{~mm}$ deflection level is 2.1 times and 3.6 times of stiffness at $10 \mathrm{~mm}$ deflection level for steel hood and aluminum hood material design, which verified increase of hood structural stiffness with vehicle pre-deformation. This study partly explained the reason why peak acceleration, HIC, and peak force increase from experiment 2 to experiment 3 (Figure 5.13 - Figure 5.14), and verified the level of vehicle predeformation is an important reason to affect head response in lateral impact scenarios.

\subsubsection{Limitation and Future Study}

Another potential impact configuration, the windshield being pre-deformed by the torso before head impact, was not observed in this study, due to a limited sample of vehicle geometry and vehicle travel speed used in this study.

Both errors and absolute errors were calculated in this study because the absolute errors can only indicate the change of magnitude, not the trend. Great variability was observed in the calculated errors, which suggested the complexity of the head response and the impact scenario dependence. Case by case study was more appropriate for this study because the sample size of 11 is small. Also, there are many factors could affect head response in the full-scale impacts, 
such as elbow impact. For example, in impact scenarios S50 and S15corner, severe elbow impacts were observed which resulted in lower head impact speeds $5.59 \mathrm{~m} / \mathrm{s}$ and $8 \mathrm{~m} / \mathrm{s}$, and thus lower HIC 333 and 394. This resulted in a different effect of the head-neck interaction with those typical lateral impact scenarios without elbow impact. In these two cases, the head-neck interactions were found to increase the HIC.

Only one order of factors was used to study the effect of those 6 major factors, and this could be a limitation because an interaction between those factors might exist. However, when switching the order between experiment 3 and experiment 4, and switching between experiment 6 and experiment 7 , the effects of factors were found to be similar.

Future study should use more vehicle models with different size and front geometry, to cover more impact configurations and to study the effect of vehicle pre-deformation on head and impactor response for different vehicles.

\subsection{Conclusion}

The reasons why the impactor test did not capture the full-scale head response were investigated. Specifically, in this chapter, at the first step, the expected major reason, the kinematical difference was matched on impactors to the full-scale head impacts to study the reason, and then the effect of all the potential major factors could cause this observation were investigated.

When there was no vehicle pre-deformation by the torso before head impact, the difference of the head impact speed was the main reason that the impactor test did not capture the full-scale head response. Conversely, when there was vehicle pre-deformation, the difference of head impact speed and vehicle pre-deformation were the two main reasons for impact tests not 
capturing the full-scale head response. The effect of other factors, such as head-neck interaction, initial angular velocity, difference on model, and head impact angle were found to be less significant.

The effects of those potential factors were impact scenario dependent. The head-neck interaction generally increased the HIC in non-lateral impacts but could decrease the HIC in lateral impacts. The vehicle pre-deformation caused more HIC increase in the lateral impact than in non-lateral impacts. 


\section{Chapter 6 Conclusions and Summary of Contributions}

\section{$\underline{\text { Summarized Conclusion }}$}

This dissertation comprehensively evaluated the biofidelity of the THUMS pedestrian model against four PMHS test data, and verified the insensitivity of enabling tissue failure on global kinematics and injury risk. Then, this dissertation tested the hypotheses that the impactor

tests cannot capture the full-scale head response, and that the vehicle design variations have different influences on the impactor response and full-scale head response. Finally the reasons for the hypothesis testing were investigated. Overall, the conclusions can be summarized as follows:

First, the morphed THUMS models captured similar responses in terms of kinematics and injuries to those of the PMHSs, while the baseline (AM50) models exhibited different response to PMHSs due to different anthropometries. Overall, the THUMS v4.01 was deemed biofidelic on the extended data type reported and could be used as an advanced injury prediction tool in vehicle-pedestrian impact research and development, despite possible model improvements on the stiffness of the spine in lateral bending and further evaluation of the soft tissue.

Second, the pedestrian kinematics was insensitive to bone fracture. Enabling tissue failure only had a local effect on kinematics of severely injured areas (lower extremities), and had little effect on the pedestrian upper body kinematics. Also, the pedestrian whole body injury risk did not show significant sensitivity on the bone quality or enabling the tissue failure.

Third, non-lateral impact caused a higher head impact speed than the lateral impact. Pedestrian head impact conditions were verified to be location-dependent. Impactor tests 
underestimated the HIC compared to the full-scale impacts, and this observation was more obvious in non-lateral impact scenarios. Full-scale impacts were more complicated than impactor tests. The head rotational-based injury risk based on BrIC was found to be insensitive to vehicle stiffness variation in this study. When there was no vehicle pre-deformation on the head impact area, vehicle stiffness design variation did have a similar impact on the impactor response and head response. However, when there was pre-deformation, vehicle stiffness design variation had different impacts on the impactor response and head response, especially in lateral impact scenarios.

Finally, when there was no vehicle pre-deformation by torso before the head impact, the difference of head impact speed was the main reason for the impactor tests not capturing the fullscale head response. When there was vehicle pre-deformation, the difference of head impact speed and vehicle pre-deformation were the main reasons for the impactor tests not capturing the full-scale head response. The effect of other factors, such as head-neck interaction, initial angular velocity, difference of the model, and head impact angle were found to be less significant. The effects of these potential factors were impact scenario dependent. The head-neck interaction generally increased the HIC in non-lateral impacts but decreased the HIC in lateral impacts. The vehicle pre-deform caused more HIC increase in the lateral impact than in the non-lateral impacts.

\section{Summarized Contribution}

Overall, the results of this dissertation suggest an important limitation of current pedestrian sub-system test procedure. The methodology and results of this dissertation provide reference for future vehicle safety design, improvement of pedestrian regulation development, 
and improvement of the biofidelity of THUMS pedestrian model. The contributions are summarized as following:

First, this dissertation used geometrically personalized models to evaluate the PFEM biofidelity, and observed better response than when using the baseline AM50 models, which suggested the anthropometry dependence of pedestrian response and the benefit of geometrically personalizing the human models for biofidelity evaluation. Also, compared to previous studies of THUMS model evaluation which focused on only the subject trajectory, this study evaluated not only the segmental velocity and acceleration, but also the time history of lower extremity strain, and tissue level injury risk using both deterministic and probabilistic methods.

Second, this dissertation verified the insensitivity of pedestrian global kinematics and whole-body injury risk on enabling the tissue failure. This study not only strengthened the conclusion on model biofidelity evaluation because of the PMHS tests that injuries were always observed, but also answered an important question that has been long considered by computational modelers of pedestrian impact.

Third, this dissertation identified an important limitation of current pedestrian sub-system test procedure. A significant difference between the component test and the full-scale pedestrian impact was the head impact area could be pre-deformed by the torso. In this type of impact configuration, vehicle stiffness design variations were found to have different impacts on the impactor response and head response. Also, this study observed that the head rotational-based injury risk based on BrIC was insensitive to vehicle stiffness variation. This suggested that the effort on vehicle design to reduce the head rotational injury risk should be on the vehicle front geometry which were found to affect the head kinematics (Liu et al. 2003; Simms and Wood 
2009; Han et al. 2012; Watanabe et al. 2012), instead of on the vehicle stiffness of head impact area.

Finally, this dissertation examined the reasons why the impactor test did not capture the full-scale head response, and found that when there was vehicle pre-deformation caused by torso before head impact, the impactor tests could not capture the full-scale head response even after matching the kinematics of impactors to full-scale head kinematics, and the major reason was that the pre-deformation could stiffen the head impact area.

\section{Recommendation for Pedestrian Regulation and Vehicle Safety Design}

Based on this study, a methodological limitation might exist on current pedestrian subsystem test procedure. That is, when there is vehicle pre-deformation by upper body before head impact, the impactor tests cannot capture the full-scale head response even with matched kinematics. Therefore, two questions might come out of this work: 1) Can we improve the testing method of current pedestrian regulation? And 2) Can we improve vehicle safety design to reduce the risk of head injury?

For the pedestrian regulation improvement, two recommendations were made here. The first is to include the vehicle pre-deformation into the testing method of regulation. Using the upper body impactor to reproduce the pedestrian upper body and head response is probably a promising method. The advantage of this method is that it can generate the pre-deformation by comparable mass of upper body. But two challenges of this method also need to be mentioned. The first challenge is that we probably not only need to create similar magnitude of vehicle predeformation, but also need to reproduce comparable time-history of vehicle pre-deformation. 
Also, if the pelvis is not included in this upper body impactor, the effect of missing vehicle predeformation caused by pelvis might need to be identified.

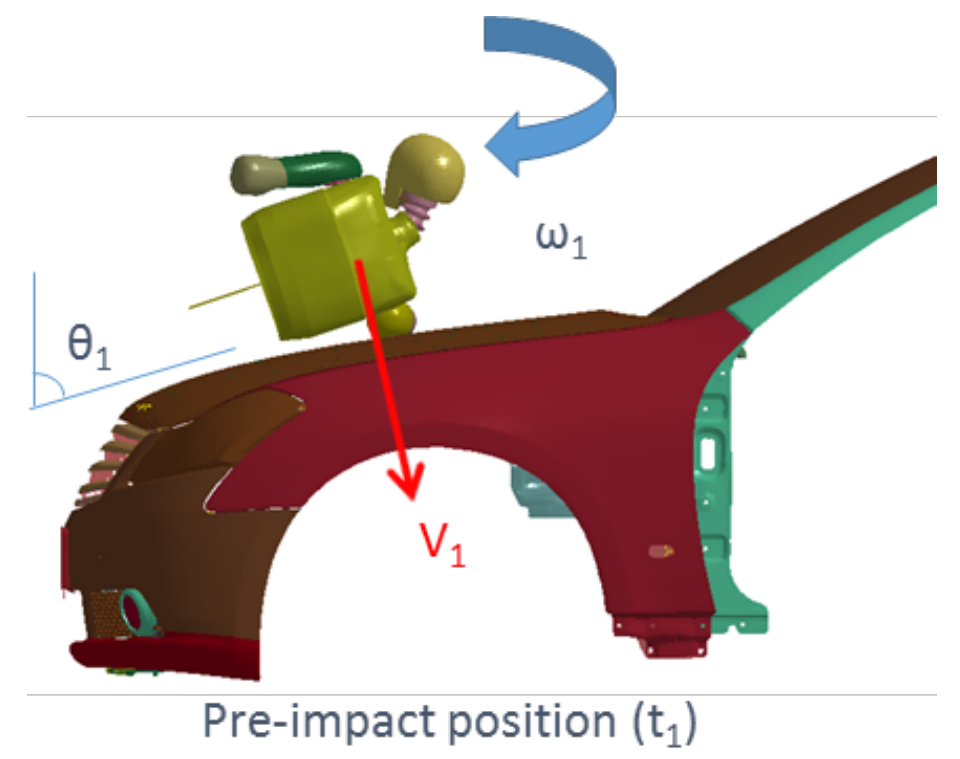

Figure 6.1 Conceptual design of upper body impactor test

Since in this study the head impact conditions were found to be dependent on pedestrian initial impact direction and head impact location, the other recommendation for the pedestrian regulation improvement is to adjust the test input conditions to better reflect realistic accident situation.

As to the pedestrian impact direction dependence, previous studies largely ignored the non-lateral pedestrian impacts (Mizuno 2003, 2005; Chen et al. 2009). However, in-depth accident investigation (Chidester et al. 2001; Neal-Strugess et al. 2007) showed non-lateral impacts account for about 30\% of pedestrian accidents. In this study, the head impact speed of non-lateral impacts is $54.2 \%$ higher than of lateral impacts, so including the non-lateral impact 
scenarios will increase $15 \%-20 \%$ of the head impact speed in testing method of pedestrian regulation according to their probably distribution in real accidents.

To consider the head impact location dependence, a potential option is to divide the vehicle surfaces into several areas by wrap around distance, and then assign different testing input conditions to those areas. The area with higher WAD could be assigned higher head impact speed according to results of this dissertation. Another fact to support higher head impact speed being assigned to areas with higher WAD is that higher vehicle travel speed generally leads higher WAD (Kawabe et al. 2012; Chen et al. 2009).

For the vehicle safety design, it is recommended that the industry not only seek vehicle performance in component test of current pedestrian regulation, but also seek the protection in pedestrian full-scale impacts. Using PFEM and vehicle FE models to optimize the vehicle design (material, thickness, boundary support, hood inner design, etc) in pedestrian full-scale impacts is a good option.

There are many advantages on using the PFEM and vehicle FE models to conduct fullscale impact simulation. First, the effect of vehicle frontal geometry on head response can be detected, not only on the translational head kinematics like head impact speed and head impact angle, but also head rotational kinematics, which was found to be important factor causing brain injury. Also, the full-scale impact simulation could reproduce more realistic vehicle deformation and thus better detect the probability of bottoming out phenomenon, which will significantly increase the head injury risk. 
In these vehicle safety designs and optimizations using full-scale impact simulation, it is recommended that different impact scenarios are considered because they could cause fundamentally different head impact response. Also, it is recommended that both translational and rotational head injury criteria could be used as the target to reduce the pedestrian head injury risk.

\section{Limitation and Future Work}

There are some limitations in this dissertation. The pedestrian head response was studied in non-lateral impact scenarios in chapter 4 and chapter 5, but the THUMS PFEM biofidelity was not evaluated in these initial impact directions in full-scale impacts, due to the lack of PMHS test data. However, at the component level, the THUMS biofidelity has been evaluated in different impact directions. All the comprehensive model evaluation studies at both full-scale and component level in the last decades, including this study, provide strong confidence on the THUMS biofidelity.

In chapter 5, only one order of factors was used to study the effect of those 6 factors, and this could be a limitation because the interaction between those factors may exist. Although when switching the order between experiment 3 and experiment 4, and switching between experiment 6 and experiment 7, the effects of factors were found to be similar, more combinations of different orders could be studied to confirm the conclusions of the effect of factors in this study. 
Future studies should use more vehicle models with different size and front geometries to cover more impact configurations, and should study the effect of vehicle pre-deformation on head and impactor responses for different vehicles.

\section{$\underline{\text { Publications }}$}

In total, this dissertation generated 10 papers that are published, under review, or in preparation, and they are listed as following:

Chen H, Crandall JR, Kerrigan J, Panzer MB. (2018) Evaluating Pedestrian Sub-system Test Procedure against Full-scale Vehicle-Pedestrian Impact Using Mathematical Models. SAE World Congress. (In preparation)

Chen H, Crandall JR, Kerrigan J, Forman J, Hartka T, Panzer MB. (2018) Effect of Factors on Pedestrian Head Response between Sub-system Test and Full-scale VehiclePedestrian Impact. SAE World Congress. (In preparation)

Chen H, Poulard D, Crandall JR, Panzer MB. (2018) Influence of Tissue Failure on Pedestrian Kinematics and Injury Risk during Vehicle-Pedestrian Impact. SAE World Congress. (In preparation)

Chen H, Poulard D, Crandall JR, Forman J, Panzer MB. (2017) Evaluation of Geometrically Personalized THUMS Pedestrian Model Response against Sedan-Pedestrian PMHS Impact Test Data. Traffic Injury Prevention. (Under review)

Chen H, Poulard D, Crandall JR, Panzer MB. (2018) Evaluation of Geometrically Personalized THUMS Pedestrian Model Response against Small City Car-Pedestrian PMHS Impact Test Data. SAE World Congress. (In preparation)

Taotao Wu, Varun Bollapragada, David Poulard, Taewung Kim, Huipeng Chen, Matt Panzer, Jeff Crandall. (2017) Evaluation of Biofidelity of THUMS Pedestrian Model under a Whole-body Impact Conditions with a Generic Sedan Buck. Traffic Injury Prevention. (Under review)

Chen, H., Bollapragada, V., Kim, T., Nie, B., Park, G. and Crandall, J.R. (2016) Improvement of lateral shoulder impact response of a multi-body pedestrian model. International Journal of Crashworthiness, pp.1-10.

Poulard D, Chen H, Panzer M. B. (2016) Geometrical personalization of pedestrian finite element models using morphing increases the biofidelity of their impact kinematics. SAE Technical Paper 2016-01-1506. 
Chen H, Poulard D, Crandall J. R, Panzer M. B. (2015) Pedestrian Response with Different Initial Positions during Impact with a Mid-Sized Sedan. In: The 24th International Technical Conference on the Enhanced Safety of Vehicles (ESV); Gothenburg, Sweden.

Poulard D, Chen H, Crandall J. R, Dziewonski T, Pedzisz M, Panzer M. B. (2015) Biofidelity Assessment of Anthropometrically-Morphed Pedestrian FE Models Using StructuralBased, Scaled PMHS Test Data. In Proceedings of the International Research Council on the Biomechanics of Injury conference (IRCOBI). 


\section{$\underline{\text { References }}$}

Akiyama, A., M. Okamoto and N. Rangarajan. Development and application of the new pedestrian dummy. International Technical Conference on the Enhanced Safety of Vehicles (ESV), Amsterdam, The Netherlands. 2001.

Akiyama, A., Yoshida, S., Matsuhashi, T., Rangarajan, N. Development of Simulation Model and Pedestrian Dummy. SAE paper No. 1999-01-0082.

Arnoux, P.J., Behr, M., Llari, M., Thollon, L. and Brunet, C. Injury criteria implementation and evaluation in FE models applications to lower limb segments. Int $\mathrm{J}$ Crashworthiness. 13(6):653-665, 2008.

Arregui-Dalmases, C. Rotational acceleration as a traumatic brain injury mechanism in pedestrian-vehicle collisions. Diss. PhD Thesis. Universidad Politécnica de Cataluña, Barcelona, Spain, 2006.

Arregui-Dalmases, C., F. J. Lopez-Valdes and M. Segui-Gomez. Pedestrian injuries in eight European countries: An analysis of hospital discharge data. Accident Analysis and Prevention 42: 1164-1171. 2010.

Automotive, T.N.O., 2001. Manual: MADYMO human body models. Delft, The Netherlands.

Banglmaier, R. F., Dvoracek-Driksna, D., Oniang'o, T. E., Haut, R. C. Axial compressive load response of the 90 flexed human tibiofemoral joint. SAE Technical Paper No. 99SC08, 1999.

Bass, C.R., Lucas, S.R., Salzar, R.S., Oyen, M.L., Planchak, C., Shender, B.S. and Paskoff, G. Failure properties of cervical spinal ligaments under fast strain rate deformations. Spine.;32(1):7-13, 2007.

Bjorklund M, Zheng Q. Development and evaluation of a pedestrian anthropomorphic test device. MSc Thesis. Dept. of Machine and Vehicle Design, Chalmers University of Technology, Göteborg, Sweden. 2001.

Block, J.P., Subramanian, S.V., Christakis, N.A. and O’Malley, A.J. Population trends and variation in body mass index from 1971 to 2008 in the Framingham Heart Study Offspring Cohort. Plos one, 8(5), p.e63217. 2013. 
Césari, D., M. Ramet, C. Cavallero, P. Billault, J. Gambarelli, G. Guérinel, J. F. Farisse and P. Bourret. Experimental Study of Pedestrian Kinematics and Injuries. International Research Council on the Biomechanics of Impact (IRCOBI), Birmingham, England. 1980.

Chen, H.P., Fu, L.X., and Zheng, H.Y. A comparative study between China and IHRA for the vehicle-pedestrian impact. SAE International Journal of Passenger Cars-Mechanical Systems 2. 1108-1115, 2009.

Chen H, Poulard D, Crandall J. R, Panzer M. B. Pedestrian Response with Different Initial Positions during Impact with a Mid-Sized Sedan. In: The 24th International Technical Conference on the Enhanced Safety of Vehicles (ESV); Gothenburg, Sweden, 2015.

Chen H, Bollapragada V, Kim T, Nie B, Park G, and Crandall J. Improvement of Lateral Shoulder Impact Response of a Multi-body Pedestrian Model. Int J Crashworthiness. 1-10. 2016.

Chidester, A.B. and Isenberg, R.A. Final report: The pedestrian crash data study. Society of Automotive Engineers. 2001.

Cowin, S.C. Bone Mechanics, Boca Raton, Florida, USA: CRC Press. 1989.

Crandall, J.R., Bhalla, K.S. and Madeley, N.J. Designing road vehicles for pedestrian protection. BMJ: British Medical Journal, 324(7346), p.1145. 2002.

Elliott, J.R., Simms, C.K. and Wood, D.P. Pedestrian head translation, rotation and impact velocity: The influence of vehicle speed, pedestrian speed and pedestrian gait. Accident Analysis \& Prevention, 45, pp.342-353. 2012.

Forman, J.L., et al. and Yarboro, S. Whole-body Response for Pedestrian Impact with a Generic Sedan Buck. Stapp Car Crash J. 59: 401-444. 2015.

Forman, Jason L., Hamed Joodaki, Ali Forghani, Patrick Riley, Varun Bollapragada, David Lessley, Brian Overby, Sara Heltzel, and Jeff Crandall. Biofidelity corridors for wholebody pedestrian impact with a generic buck. In IRCOBI Conf, vol. 49, pp. 356-372. 2015.

Fredriksson, R., Shin, J. and Untaroiu, C.D. Potential of pedestrian protection systems-a parameter study using finite element models of pedestrian dummy and generic passenger vehicles. Traffic injury prevention, 12(4), pp.398-411. 2011.

Fredriksson, Rikard, Yngve Håland, and Jikuang Yang. Evaluation of a new pedestrian head injury protection system with a sensor in the bumper and lifting of the bonnet's rear part. Society of Automotive Engineers, 2001. 
Funk, J. R., Srinivasan, S. C., Crandall, J. R., Khaewpong, N., Eppinger, R. H., Jaffredo, A. S., Petit, P. Y. The effects of axial preload and dorsiflexion on the tolerance of the ankle/subtalar joint to dynamic inversion and eversion. Stapp Car Crash J. 46:245-265. 2002.

Fung, Y.C. Biomechanics: Mechanical Properties of Living Tissues, New York: Springer-Verlag. 1993.

Gabler, L.F., Crandall, J.R. and Panzer, M.B. Assessment of kinematic brain injury metrics for predicting strain responses in diverse automotive impact conditions. Annals of biomedical engineering, 44(12), pp.3705-3718. 2016.

Gadd, Charles W. Use of a weighted-impulse criterion for estimating injury hazard. No. 660793. SAE Technical Paper, 1966.

Gehre, C., Gades, H., and Wernicke, P. Objective Rating of Signals using Test and Simulation Responses. 21nd ESV Conference. Stuttgart, Germany. 2009.

Erdmann, W.S. Geometry and inertia of the human body-review of research. Acta of Bioengineering and biomechanics, 1(1), pp.23-35. 1999.

Golman, A.J., Danelson, K.A., Miller, L.E. and Stitzel, J.D. Injury prediction in a side impact crash using human body model simulation. Accid Anal Prev.64: 1-8. 2014.

Gordon, C.C., Blackwell, C.L., Bradtmiller, B., Parham, J.L., Barrientos, P., Paquette, S.P., Corner, B.D., Carson, J.M., Venezia, J.C., Rockwell, B.M. and Mucher, M., 2014. 2012 Anthropometric Survey of US Army Personnel: Methods and Summary Statistics (No. NATICK/TR-15/007). ARMY NATICK SOLDIER RESEARCH DEVELOPMENT AND ENGINEERING CENTER MA.

Got, C., A. Patel, A. Fayon, C. Tarriere, and G. Walfisch. Results of experimental head impacts on cadavers: the various data obtained and their relations to some measured physical parameters. No. 780887. SAE Technical Paper, 1978.

Gunji, Y., Okamoto, M. and Takahashi, Y. Examination of human body mass influence on pedestrian pelvis injury prediction using a human FE model. In Proceedings of the International Research Council on the Biomechanics of Injury conference. 40:316-327. 2012.

Gupta, Vishal. Pedestrian Head Protection during Car To Pedestrian Accidents: In The Event Of Primary Impact With Vehicle And Secondary Impact With Ground. PhD diss., Ph. D., Wayne State University, Detroit, MI, 2014. 
Hamdane, H., Serre, T., Anderson, R. and Yerpez, J. Accident simulation and reconstruction for enhancing pedestrian safety: issues and challenges. In ESAR 2014-6th International Conference: Expert Symposium on Accident Research (p. 11P). 2014, June.

Han Y, Yang J, Mizuno K, Matsui Y. Effects of Vehicle Impact Velocity, Vehicle FrontEnd Shapes on Pedestrian Injury Risk. Traffic Inj Prev.13:5:507-518. 2012.

Hardy, B.J., Lawrence, G.J.L., Knight, I.M., Simmons, I.C.P., Carroll, J.A., Coley, G. and Bartlett, R.S. A study of possible future developments of methods to protect pedestrians and other vulnerable road users. TRL PROJECT REPORT. 2007.

Hardy, R. APROSYS - Final Report for the Work on "Pedestrian and Pedal Cyclist Accidents" (SP3)". Cranfield Impact Centre, AP-90-0003. 2009.

Harruff, R. C., A. Avery and A. S. Alter-Pandya. Analysis of Circumstances and Injuries in 217 Pedestrian Traffic Fatalities. Accident Analysis and Prevention 30(1): 11-20. 1998.

Hayamizu, N., Sakuma, S., Hayashi, S., Kozato, A. Experimental Study of Child Pedestrian Injury - Development of Prototype 6-Years-Old Pedestrian Dummy, proc. of 2002 JSAE Annual Congress. 2002.

Holbourn, A. H. S. Mechanics of head injuries. Lancet 242:438-441, 1943.

IRTAD. Road Safety Annual Report 2014. International Traffic Safety Data and Analysis Group (IRTAD), Organisation for Economic Co-operation and Development (OECD). 2014.

Ishikawa, H., Kajzer, J. and Schroeder, G. Computer simulation of impact response of the human body in car-pedestrian accidents (No. 933129). SAE Technical Paper. 1993.

Iwamoto, M., Kisanuki, Y., Watanabe, I., Furusu, K., Miki, K. and Hasegawa, J. Development of a finite element model of the total human model for safety (THUMS) and application to injury reconstruction. In Proceedings of the International Research Council on the Biomechanics of Injury conference. 30:1-12. 2002.

Ivarsson, J., Lesley, D., Kerrigan, J., Bhalla, K., Bose, D., Crandall, J. R., Kent, R. W. Dynamic response corridors and injury thresholds of the pedestrian lower extremities. In Proceedings of the International Research Council on the Biomechanics of Injury conference. Vol. 32. 2004.

Kalliske, I. and Friesen, F. Improvments to Pedestrian Protection as Exemplified on a Standard-sized Car, Proc. 17th Int. Technical Conf. on Enhanced Safety Vehicle. 2001. 
Kalos, M.H. and Whitlock, P.A. Monte carlo methods. John Wiley \& Sons. 2008.

Kawabe, Yoshiko, Toshiyuki Asai, Daisuke Murakami, Chinmoy Pal, and Tomosaburo Okabe. Different factors influencing post-crash pedestrian kinematics. SAE International journal of passenger cars-mechanical systems 5, no. 2012-01-0271: 214-230. 2012.

Kent, R., Patrie, J. Chest deflection tolerance to blunt anterior loading is sensitive to age but not load distribution. Forensic Sci Int. 149(2):121-128. 2005.

Kerkeling, Christoph, Joachim Schäfer, and Grace-Mary Thompson. Structural hood and hinge concepts for pedestrian protection. In Proc. 17th Int. Tech. Conf. ESV, pp. 4-7. 2005.

Kerrigan, Jason Robert. A computationally efficient mathematical model of the pedestrian Lower extremity. PhD diss., Ph. D., University of Virginia, Charlottesville, VA, 2008.

Kerrigan, J.R., Crandall, J.R. and Deng, B. A comparative analysis of the pedestrian injury risk predicted by mechanical impactors and post mortem human surrogates. Stapp car crash journal, 52, p.527. 2008.

Kerrigan, J. R., Drinkwater, D. C., Kam, C. Y., Murphy, D. B., Ivarsson, B. J., Crandall, J. R., Patrie, J. Tolerance of the human leg and thigh in dynamic latero-medial bending. Int $\mathrm{J}$ Crashworthiness. 9(6):607-623. 2004.

Kerrigan J. R., Crandall J. R, Deng B. Pedestrian kinematic response to mid-sized vehicle impact. Int J Veh Saf. 2(3):221-240. 2007.

Kerrigan, J. R., Arregui-Dalmases, C., Foster, J., Crandall, J. R., Rizzo, A. Pedestrian injury analysis: field data vs. laboratory experiments. In Proceedings of the International Research Council on the Biomechanics of Injury conference. 40:672-689. 2012.

Kessler, J., and M. Monk. "NHTSA pedestrian head injury mitigation research programStatus report." In Experiment Safety Vehicles Conference, pp. 1226-1236. 1991.

Kuppa, S. Injury criteria for side impact dummies. Washington, DC: National Transportation Biomechanics Research Center, National Highway Saftey Administration, US DOT, 67. 2004.

Kuppa, S., Eppinger, R. H., McKoy, F., Nguyen, T., Pintar, F. A., Yoganandan, N. Development of Side Impact Thoracic Injury Criteria and Their Application to the Modified ES2 Dummy with Rib Extensions (ES-2re). Stapp Car Crash J. 47:189-210. 2003. 
Lau, G., E. Seow and E. S. Lim. A review of pedestrian fatalities in Singapore from 1990 to 1994. Annals of the Academy of Medicine, Singapore 27(6): 830-837. 1998.

Leijdesdorff, H. A., J. T. J. M. van Dijck, P. Krijnen, C. L. A. M. Vleggeert-Lankamp and I. B. Schipper. Injury Pattern, Hospital Triage, and Mortality of 1250 Patients with Severe Traumatic Brain Injury Caused by Road Traffic Accidents. Journal of Neurotrauma 31: 459-465. 2014.

Li, G., Yang, J., Simms, C. The influence of gait stance on pedestrian lower limb injury risk. Accid Anal Prev. 85: 83-92. 2015.

Liu, Qi, Yong Xia, Qing Zhou, and Jenne-Tai Wang. Design analysis of a sandwich hood structure for pedestrian protection. In The 21st International Technical Conference on the Enhanced Safety of Vehicles Conference. Stuttgart, Germany. 2009.

Liu, Xuejun. Mathematical Simulation of Vehicle-Pedestrian Collisions: Influence of Vehicle Impact Speed and Front-End Structure on the Dynamic Responses of Child and Adult Pedestrians. PhD diss., Ph. D. Chalmers University of Technology, 2003.

X.J. Liu, J.K. Yang, and L"ovsund, A study of influences of vehicle speed and front structure on pedestrian impact responses using mathematical models, Traffic Injury Prev. 3 (2002), pp. 31-42.

MacLaughlin, Thomas F., John F. Wiechel, and Dennis A. Guenther. Head Impact Reconstruction-HIC Validation and Pedestrian Injury Risk. No. 930895. SAE Technical Paper, 1993.

Maeno T, Hasegawa J. Development of a finite element model of the total human model for safety (THUMS) and application to car-pedestrian impacts. The 17th International Technical Conference on the Enhanced Safety of Vehicles (ESV). Amsterdam, The Netherlands. 2001.

Matsui, Y., Doi, T., Oikawa, S. and Ando, K. Features of fatal pedestrian injuries in vehicle-to-pedestrian accidents in Japan. SAE International journal of transportation safety, 1(2013-01-0777), pp.297-308. 2013.

Matsui, Y. and Tanahashi, M. Development of JAMA—JARI pedestrian headform impactor in compliance with ISO and IHRA standards. International Journal of Crashworthiness, 9(2), pp.129-139. 2004.

McIntosh, A. S., D. Kallieris, R. Mattern, and E. Miltner. Head and neck injury resulting from low velocity direct impact. No. 933112. SAE Technical Paper, 1993. 
Meyer, E.G., Wei, F., Button, K., Powell, J.W. and Haut, R.C. Determination of ligament strain during high ankle sprains due to excessive external foot rotation in sports. In Proceedings of the International Research Council on the Biomechanics of Injury conference. 40:277-288. 2012.

Mizuno, Koji, and Janusz Kajzer. Head injuries in vehicle-pedestrian impact. No. 200001-0157. SAE Technical Paper, 2000.

Mizuno, Y. Summary of IHRA safety WG activities (2003)-proposed test methods to evaluate pedestrian protection afforded by passenger cars. Proceedings of the 18th International Technical Conference on the Enhanced Safety of Vehicles, Nagoya, Japan, Paper. No. 580. 2003.

Mizuno, Y., 2005. Summary of IHRA Pedestrian Safety WG Activities (2005)-Proposed test methods to evaluate pedestrian protection afforded by passenger cars. In Proceedings: International Technical Conference on the Enhanced Safety of Vehicles (Vol. 2005, pp. 15p-15p). National Highway Traffic Safety Administration.

Mo, F., Arnoux, P. J., Cesari, D., Masson, C. Investigation of the injury threshold of knee ligaments by the parametric study of car-pedestrian impact conditions. Saf sci. 62: 58-67. 2014.

Naci H, Chisholm D, Baker T. D. Distribution of road traffic deaths by road user group: a global comparison. Inj Prev. 15(1): 55-59. 2009.

Nagatomi, Kaoru, Ken Hanayama, Tatsuya Ishizaki, Sakae Sasaki, and Kazuo Matsuda. Development and full-scale dummy tests of a pop-up hood system for pedestrian protection. In Proceedings of the 19th International Technical Conference "Enhanced Safety of Vehicles" ESV, Washington, pp. 05-0113. 2005.

Nahum, A.M. and Melvin, J.W. Accidental Injury Biomechanics and Prevention, New York: Springer-Verlag. 2001.

Neale, M.S., Hardy, B.J. and Lawrence, G.J. Development and evaluation of a biofidelic shoulder for the IHRA (JARI) pedestrian model. In Proceedings of the 19th international technical conference on the Enhanced Safety of Vehicles. Paper No. 05-0096. 2005.

Neal-Sturgess, C.E., Carter, E., Hardy, R., Cuerden, R., Guerra, L. and Yang, J. APROSYS European in-depth pedestrian database. Age, 91, p.95. 2007.

Nie, B. and Zhou, Q. Can new passenger cars reduce pedestrian lower extremity injury? A review of geometrical changes of front-end design before and after regulatory efforts. Traffic injury prevention, (just-accepted), pp.00-00. 2016. 
Niebuhr, T., Junge, M. and Rosen, E. Pedestrian injury risk and the effect of age. Accident Analysis \& Prevention, 86, pp.121-128. 2016.

Okamoto, Y., Sugimoto, T., Enomoto, K. and Kikuchi, J. Pedestrian head impact conditions depending on the vehicle front shape and its construction--full model simulation. Traffic injury prevention, 4(1), pp.74-82. 2003.

Olver, J. H., J. L. Ponsford and C. A. Curran. Outcome following traumatic brain injury: a comparison between 2 and 5 years after injury. Brain Injury 10(11): 841-848. 1996.

Paas, Ruth. Head kinematics in car-pedestrian crashes: The influence of sliding, spine bending, elbow and shoulder impacts. Chalmers University of Technology, 2015.

Paas R, Davidsson J, Masson C, Sander U, Brolin K, Yang JK. Pedestrian Shoulder and Spine Kinematics in Full-Scale PMHS Tests for Human Body Model Evaluation. Proceedings of IRCOBI Conference, 730-750. Dublin, Ireland. 2012.

Paas, R., Masson, C., Davidsson, J. Head boundary conditions in pedestrian crashes with passenger cars: six-degrees-of-freedom post-mortem human subject responses. Int J Crashworthiness. 1-13. 2015.

Paas, R., Davidsson, J. and Brolin, K., Head Kinematics and Shoulder Biomechanics in Shoulder Impacts Similar to Pedestrian Crashes-A THUMS Study. Traffic Inj Prev. 16(5):498506. 2015.

Peng, Y., Deck, C., Yang, J. and Willinger, R. Effects of pedestrian gait, vehicle-front geometry and impact velocity on kinematics of adult and child pedestrian head. International Journal of Crashworthiness, 17(5), pp.553-561. 2012.

Penman, A.D. and Johnson, W.D. The changing shape of the body mass index distribution curve in the population: implications for public health policy to reduce the prevalence of adult obesity. Prev Chronic Dis, 3(3), p.A74. 2006.

Poulard D, Chen H, Crandall J. R, Dziewonski T, Pedzisz M, Panzer M. B. Biofidelity Assessment of Anthropometrically-Morphed Pedestrian FE Models Using Structural-Based, Scaled PMHS Test Data. In Proceedings of the International Research Council on the Biomechanics of Injury conference. 2015.

Poulard D, Chen H, Panzer M. B. Geometrical personalization of pedestrian finite element models using morphing increases the biofidelity of their impact kinematics. SAE Technical Paper 2016-01-1506. 2016. 
Pritz, H. B., E. B. Weis and J. T. Herridge. Body-vehicle interaction: experimental study, Volume I. National Highway Traffic Safety Administration (NHTSA), Washington, D.C., USA, 1975.

Quinn, K.P. and Winkelstein, B.A. Cervical facet capsular ligament yield defines the threshold for injury and persistent joint-mediated neck pain. J Biomech. 40(10):2299-2306. 2007.

Rosén, E. and Sander, U., 2009. Pedestrian fatality risk as a function of car impact speed. Accident Analysis \& Prevention, 41(3), pp.536-542.

Roudsari, Bahman S., Charles N. Mock, and Robert Kaufman. An evaluation of the association between vehicle type and the source and severity of pedestrian injuries. Traffic Injury Prevention 6, no. 2: 185-192. 2005.

Roudsari, B.S., Mock, C.N., Kaufman, R., Grossman, D., Henary, B.Y. and Crandall, J. Pedestrian crashes: higher injury severity and mortality rate for light truck vehicles compared with passenger vehicles. Injury Prevention, 10(3), pp.154-158. 2004.

Searson, D.J., Anderson, R.W.G., Ponte, G., and van den Berg A.L. Headform impact test performance of vehicles under the GTR on pedestrian safety (CASR072), Centre for Automotive Safety Research, Adelaide. 2009.

Simms, C. and Wood, D. Pedestrian and cyclist impact: a biomechanical perspective (Vol. 166). Springer Science Business Media. 2009.

Simms, C.K. and Wood, D.P. Effects of pre-impact pedestrian position and motion on kinematics and injuries from vehicle and ground contact. International Journal of Crashworthiness, 11(4), pp.345-355. 2006.

Snedeker J.G, Muser M.H, Walz F.H. Assessment of Pelvis and Upper Leg Injury Risk in Car-Pedestrian Collisions: Comparison of Accident Statistics, Impactor Tests and a Human Body Finite Element Model. Stapp Car Crash J. 47:437-457. 2003.

Schroeder, G., K. Fukuyama, K. Yamazaki, K. Kamiji and T. Yasuki. Injury Mechanism of Pedestrians Impact Test with a Sport-Utility Vehicle and Mini-Van. International Research Council on the Biomechanics of Injury (IRCOBI), Bern, Switzerland. 2008.

Snedeker, J. G., Walz, F. H., Muser, M. H., Lanz, C., Schroeder, G. Assessing femur and pelvis injury risk in car-pedestrian collisions: comparison of full body PMTO impacts, and a human body finite element model. International Technical of Conference on the Enhanced Safety of Vehicles. Lyon, France. 2005. 
Soni, A., Robert, T. and Beillas, P., 2013. Effects of Pedestrian Pre-Crash Reactions on Crash Outcomes during Multi-body Simulations. In IRCOBI Conference Proceedings (No. IRC13-92).

Soni, A., Robert, T., Rongiéras, F. and Beillas, P. Observations on pedestrian pre-crash reactions during simulated accidents. Stapp car crash journal, 57, p.157. 2013.

Subit D, Kerrigan J, Crandall J. R, Fukuyama K, Yamazaki K, Kamiji, Yasuki T. Pedestrian-vehicle interaction: kinematics and injury analysis of four full scale tests. In Proceedings of the International Research Council on the Biomechanics of Injury conference. 36: 275-294. 2008.

Sugimoto, T. and K. Yamazaki. First results from the JAMA human body model project. Enhanced Safety of Vehicles (ESV), Washington, D.C. 2005.

Takhounts, E. G., M. J. Craig, K. Moorhouse, J. McFadden, and V. Hasija. Development of brain injury criteria (BrIC). Stapp Car Crash J. 57:243-266, 2013.

Takhounts, E. G., R. H. Eppinger, J. Q. Campbell, R. E. Tannous, et al. On the development of the SIMon finite element head model. Stapp Car Crash J. 47:107, 2003.

Takhounts, E. G., V. Hasija, S. A. Ridella, S. Rowson, and S. M. Duma. Kinematic rotational brain injury criterion (BRIC). In: Proceedings of the 22nd Enhanced Safety of Vehicles Conference. Paper no. 11-0263, 2011.

Takhounts, E. G., S. A. Ridella, V. Hasija, R. E. Tannous, J. Q. Campbell, D. Malone, K. Danelson, J. Stitzel, S. Rowson, and S. Duma. Investigation of traumatic brain injuries using the next generation of simulated injury monitor (SIMon) finite element head model. Stapp Car Crash J 52:1-31, 2008.

Gennarelli, T. A., A. K. Ommaya and L. E. Thibault. Comparison of Translational and Rotational Head Motions in Experimental Cerebral Concussion. 15th Stapp Car Crash Conference, Coronado, CA. 1971.

Gennarelli, T. A., L. E. Thibault and A. K. Ommaya. Pathophysiologic Responses to Rotational and Translational Accelerations of the Head. 16th Stapp Car Crash Conference, Detroit, MI. 1972.

T. Gennarelli, L. Thibault, J. Adams, D. Graham, C. Thompson, R. Marcincin. Diffuse axonal injury and traumatic coma in the primate. Annals of Neurology. Page: 564-574. 1982. 
Gennarelli, T. A., and L. E. Thibault. Clinical rationale for a head injury angular acceleration criterion. Head injury mechanisms: The need for an angular acceleration criterion, Association for the Advancement of Automotive Medicine 5-8. 1989.

Toyota Motor Corporation. THUMS Users Manual Version 4.0. 2011.

Trajkovski, A., Omerović, S., Hribernik, M. and Prebil, I. Failure Properties and Damage of Cervical Spine Ligaments, Experiments and Modeling. Journal Biomech Eng. 136(3):031002. 2014.

United Nations Economic and Social Council. Proposal for amendments to global technical regulation no. 9. ECE/TRANS/WP.29/GRSP/2010/2 (2010). Available at: http://www.unece.org/trans/main/wp29/wp29wgs/wp29grsp/grsp2010.html.

Untaroiu, Costin Daniel. Development and Validation of a Finite Element Model of Human Lower Limb: Including Detailed Geometry, Physical Material Properties, and Component Validations for Pedestrian Injuries. PhD diss., Ph. D., University of Virginia, Charlottesville, VA, 2005.

Untaroiu C et al. Correlation of strain and loads measured in the long bones with observed kinematics of the Lower Limb during Vehicle-Pedestrian Impacts. Stapp Car Crash J. 51:433. 2007.

Untaroiu, C.D., Meissner, M.U., Crandall, J.R., Takahashi, Y., Okamoto, M. and Ito, O. Crash reconstruction of pedestrian accidents using optimization techniques. International Journal of Impact Engineering, 36(2), pp.210-219. 2009.

Untaroiu, Costin D., Jaeho Shin, and Jeff R. Crandall. A design optimization approach of vehicle hood for pedestrian protection. International Journal of Crashworthiness 12, no. 6 (2007): 581-589. 2007.

Untaroiu, Costin D., Jacob B. Putnam, Jeremy Schap, Matt L. Davis, and F. Scott Gayzik. Development and Preliminary Validation of a 50th Percentile Pedestrian Finite Element Model. In ASME 2015 International Design Engineering Technical Conferences and Computers and Information in Engineering Conference, pp. V003T01A004-V003T01A004. American Society of Mechanical Engineers, 2015.

Vavalle, N.A., Jelen, B.C., Moreno, D.P., Stitzel, J.D. and Gayzik, F.S. An evaluation of objective rating methods for full-body finite element model comparison to PMHS tests. Traffic Inj Prev. 14(sup1): S87-S94. 2013. 
Vezin, P. and J. P. Verriest Development of a set of numerical human models for safety. Enhanced Safety of Vehicles (ESV), Washington D.C. 2005.

Wanke, Thomas, Grace Thompson, and Christoph Kerkeling. Pedestrian measures for the Opel Zafira II. In Experimental Safety Vehicles Conference. 2005.

Watanabe R, Katsuhara T, Miyazaki H, Kitagawa Y, Yasuki T. Research of the Relationship of Pedestrian Injury to Collision Speed, Car-type, Impact Location and Pedestrian Sizes using Human FE model (THUMS Version 4). Stapp Car Crash J. 56:269- 321. 2012.

WHO. Pedestrian safety: a road safety manual for decision-makers and practitioners. World Health Organization (WHO), Geneva, Switzerland, ISBN: 978924150535 2. 2013.

World Health Organization. Violence, Injury Prevention and World Health Organization, 2013. Global status report on road safety 2013: supporting a decade of action. World Health Organization.

Yang, J. Review of injury biomechanics in car-pedestrian collisions. International journal of vehicle safety, 1(1-3), 100-117. 2005.

Yao, J., Yang, J., and Otte, D. Head injuries in child pedestrian accidents-in-depth case analysis and reconstructions. Traffic injury prevention, 8(1), 94-100. 2007.

Yao, J. Investigations of Head impact dynamics, Injury Mechanisms and countermeasures in car to pedestrian accidents. PhD thesis. Chalmers University of Technology, Göteborg, Sweden. 2010.

Yasuki T, Yamamae Y. Validation of Kinematics and Lower Extremity Injuries Estimated by Total Human Model for Safety in SUV to Pedestrian Impact Test. Journal of Biomechanical Science and Engineering. 5(4): 340-356. 2010.

Yoganandan, N., Zhang, J. and Pintar, F.A. Force and acceleration corridors from lateral head impact. Traffic injury prevention, 5(4), pp.368-373. 2004.

Zhang, Q., Kindig, M., Li, Z., Crandall, J. R., Kerrigan, J. R. Development of structural and material clavicle response corridors under axial compression and three point bending loading for clavicle finite element model validation. J Biomech. 47(11):2563-2570. 2014. 